\title{
Transformada Imagem-Floresta com Funções de Conexidade Não Suaves: Pesos Adaptativos, Polaridade de Borda, e Restrições de Forma
}

Lucy Alsina Choque Mansilla

\author{
DiSSERTAÇÃO APRESENTADA \\ $\mathrm{AO}$ \\ Instituto de Matemática e Estatística \\ DA \\ Universidade DE SÃo PAUlo \\ PARA \\ OBTENÇÃO DO TÍTULO \\ DE \\ Mestre EM CiÊnCIAS
}

\author{
Programa: Ciência da Computação \\ Orientador: Prof. Dr. Paulo André Vechiatto de Miranda
}

Durante o desenvolvimento deste trabalho o autor recebeu auxílio financeiro da CAPES e da FAPESP (2012/06911-2).

São Paulo, fevereiro de 2014 


\title{
Transformada Imagem-Floresta com Funções de Conexidade Não Suaves: Pesos Adaptativos, Polaridade de Borda, e Restrições de Forma
}

\begin{abstract}
Esta versão da dissertação contém as correções e alterações sugeridas pela Comissão Julgadora durante a defesa da versão original do trabalho, realizada em 26/02/2014. Uma cópia da versão original está disponível no Instituto de Matemática e Estatística da Universidade de São Paulo.
\end{abstract}

Comissão Julgadora:

- Prof. Dr. Paulo André Vechiatto de Miranda (orientador) - IME-USP

- Prof. Dr. Marcel Parolin Jackowski - IME-USP

- Prof. Dr. João Paulo Papa - UNESP 


\section{Agradecimentos}

Agradeço a Deus pela saúde, força e guia constante. Aos meus pais, Antonio e Alsina, bem como aos demais membros da minha família: Ysabel, Miriam, Mary, Yul e Luisito. Muito obrigada pelo amor, apoio, compreensão e incentivo. Vocês são o motivo pelo qual avanço na realização dos meus sonhos.

Agradeço profundamente ao meu orientador, professor Paulo André Vechiatto de Miranda pela disposição contínua, ensinamentos, paciência, bom humor, e essa energia que contagia e impulsiona a progredir na carreira, e principalmente por acreditar em mim dando-me a oportunidade de pesquisar nesta área.

Gostaria de agradecer aos professores Nina Sumiko Tomita Hirata, Marcel Parolin Jackowski e João Paulo Papa por todas as sugestões e correções dadas no exame de qualificação e na defesa da dissertação. As idéias sugeridas foram fundamentais para a conclusão deste trabalho.

Gostaria de agradecer também aos professores Ernesto Cuadros, César Beltrán e Juan Carlos Gutiérrez pelo apoio antes de começar o mestrado.

À Jihan Zoghbi pelas dicas e muitos ensinamentos. Ao Jesús Mena, à Rosario Medina, ao Frank Julca e ao Leandro Ticlia, pela boa recepção e disposição no início do mestrado. Obrigada pela amizade, apoio e companheirismo.

Aos meus amigos: Marisol, Edwin, Evelyn e Andréa, obrigada pelo apoio e por tudo o que compartilhamos. Aos meus amigos do IME-USP: Silvia, Miguel, Edu, Erika, Mariela, Talita, Amanda P., Gesiele, Maysa, David, Jorge, Leissi, Amanda G., Lulu, Alfonso, Juan G., Hans, Waldir, Sabrina, Juan V., Sérgio, Paulo, Fernando, Suzana, Igor e Wonder. Bem como a todos os meus demais amigos com os quais partilhei de diversas experiências ao longo de minha jornada. Obrigada pela amizade, compreensão e momentos compartilhados.

Finalmente, agradeço à CAPES e à FAPESP pelo apoio financeiro durante meu mestrado, o que permitiu minha dedicação integral a essa pesquisa. 


\section{Resumo}

\section{CHOQUE-MANSILLA, LUCY A. Transformada Imagem-Floresta com Funções de Cone-}

xidade Não Suaves: Pesos Adaptativos, Polaridade de Borda, e Restrições de Forma.

2014. 123 f. Dissertação (Mestrado) - Instituto de Matemática e Estatística, Universidade de São Paulo, São Paulo, 2014.

Segmentar uma imagem consiste em particioná-la em regiões relevantes para uma dada aplicação, como para isolar um objeto de interesse no domínio de uma imagem. A segmentação é um dos problemas mais fundamentais e desafiadores em processamento de imagem e visão computacional. Ela tem desempenhado um papel importante, por exemplo, na pesquisa em neurologia, envolvendo imagens de Ressonância Magnética (RM), para fins de diagnóstico e tratamento de doenças relacionadas com alterações na anatomia do cérebro humano.

Métodos de segmentação baseados na transformada imagem-floresta (IFT, Image Foresting Transform), com funções de conexidade suaves, possuem resultados ótimos, segundo o critério da otimalidade dos caminhos descrito no artigo original da IFT, e têm sido usados com sucesso em várias aplicações, como por exemplo na segmentação de imagens RM de 1.5 Tesla. No entanto, esses métodos carecem de restrições de regularização de borda, podendo gerar segmentações com fronteiras muito irregulares e indesejadas. Eles também não distinguem bem entre bordas similares com orientações opostas, e possuem alta sensibilidade à estimativa dos pesos das arestas do grafo, gerando problemas em imagens com efeitos de inomogeneidade.

Nesse trabalho são propostas extensões da IFT, do ponto de vista teórico e experimental, através do uso de funções de conexidade não suaves, para a segmentação interativa de imagens por região. A otimalidade dos novos métodos é suportada pela maximização de energias de corte em grafo, ou como o fruto de uma sequência de iterações de otimização de caminhos em grafos residuais.

Como resultados principais temos: O projeto de funções de conexidade mais adaptativas e flexíveis, com o uso de pesos dinâmicos, que permitem um melhor tratamento de imagens com forte inomogeneidade. O uso de grafos direcionados, de modo a explorar a polaridade de borda dos objetos na segmentação por região, e o uso de restrições de forma que ajudam a regularizar a fronteira delineada, favorecendo a segmentação de objetos com formas mais regulares. Esses avanços só foram possíveis devido ao uso de funções não suaves. Portanto, a principal contribuição desse trabalho consiste no suporte teórico para o uso de funções não suaves, até então evitadas na literatura, abrindo novas perspectivas na pesquisa de processamento de imagens usando grafos.

Palavras-chave: transformada imagem-floresta, funções de conexidade não suaves, segmentação interativa de imagens, segmentação por corte em grafo, caminhos mais curtos, convexidade geodésica em estrela, watersheds, conexidade fuzzy. 


\section{Abstract}

\section{CHOQUE-MANSILLA, LUCY A. Image Foresting Transform with Non-smooth Connec-}

tivity Functions: Adaptive Weights, Boundary Polarity, and Shape Constraints. 2014. 123 f. Dissertação (Mestrado) - Instituto de Matemática e Estatística, Universidade de São Paulo, São Paulo, 2014.

Segmenting an image consist in to partition it into relevant regions for a given application, as to isolate an object of interest in the domain of an image. Segmentation is one of the most fundamental and challenging problems in image processing and computer vision. It has played an important role, for example, in neurology research, involving images of Magnetic Resonance (MR), for the purposes of diagnosis and treatment of diseases related to changes in the anatomy of the human brain.

Segmentation methods based on the Image Foresting Transform (IFT), with smooth connectivity functions, have optimum results, according to the criterion of path optimality described in the original IFT paper, and have been successfully used in many applications as, for example, the segmentation of MR images of 1.5 Tesla. However, these methods present a lack of boundary regularization constraints and may produce segmentations with quite irregular and undesired boundaries. They also do not distinguish well between similar boundaries with opposite orientations, and have high sensitivity to the arc-weight estimation of the graph, producing poor results in images with strong inhomogeneity effects.

In this work, we propose extensions of the IFT framework, from the theoretical and experimental points of view, through the use of non-smooth connectivity functions for region-based interactive image segmentation. The optimality of the new methods is supported by the maximization of graph cut energies, or as the result of a sequence of paths optimizations in residual graphs.

We have as main results: The design of more adaptive and flexible connectivity functions, with the use of dynamic weights, that allow better handling of images with strong inhomogeneity. The use of directed graphs to exploit the boundary polarity of the objects in region-based segmentation, and the use of shape constraints that help to regularize the segmentation boundary, by favoring the segmentation of objects with more regular shapes. These advances were only made possible by the use of non-smooth functions. Therefore, the main contribution of this work is the theoretical support for the usage of non-smooth functions, which were until now avoided in literature, opening new perspectives in the research of image processing using graphs.

Keywords: image foresting transform, non-smooth connectivity functions, interactive image segmentation, graph-cut segmentation, shortest paths, geodesic star convexity, watersheds, fuzzy connectedness. 


\section{Sumário}

Lista de Abreviaturas $\quad$ xi

Lista de Símbolos $\quad$ xiii

Lista de Figuras $\quad$ Xv

$\begin{array}{lc}\text { Lista de Tabelas } & \text { xxi }\end{array}$

1 Introdução $\quad \mathbf{1}$

1.1 Pesos adaptativos . . . . . . . . . . . . . . . . . . . 3

1.2 Polaridade de borda $\ldots \ldots \ldots \ldots \ldots \ldots \ldots \ldots$

1.3 Restrições de forma . . . . . . . . . . . . . . . . . . . . . . . . . . . . . . . . . . . .

1.4 Organização do Trabalho . . . . . . . . . . . . . . . . . . . . . 7

2 Conceitos 9

2.1 Imagem digital $\ldots \ldots \ldots \ldots \ldots \ldots \ldots \ldots$

2.1 .1 Imagem em tons de cinza . . . . . . . . . . . . . . . . . . . 9

2.1 .2 Imagem multidimensional . . . . . . . . . . . . . . . . . . 10

2.1 .3 Imagem multibanda . . . . . . . . . . . . . . . . . . . . . . . 10

2.1 .4 Imagem de rótulos . . . . . . . . . . . . . . . . . . . . 10

2.2 Noções de grafos . . . . . . . . . . . . . . . . . . . . . . . . . . . 10

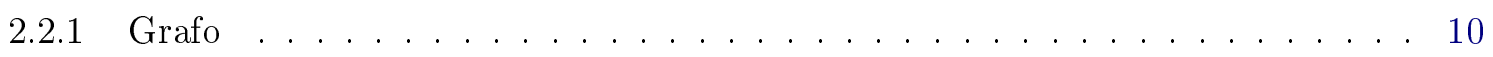

2.2 .2 Grafo direcionado . . . . . . . . . . . . . . . . . . . . 11

2.2 .3 Grafo ponderado . . . . . . . . . . . . . . . . 11

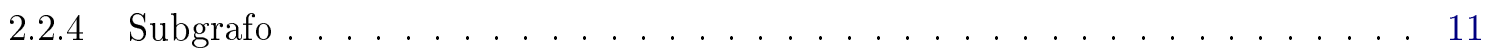

2.2 .5 Relação de adjacência . . . . . . . . . . . . . . . . . . . . . . . . . . . . . . . . . . . . . . 11

2.2 .6 Caminho . . . . . . . . . . . . . . . . . . . . . . 11

2.2 .7 Ciclo . . . . . . . . . . . . . . . . . . . . 11

2.2 .8 Grafo conexo . . . . . . . . . . . . . . . . . . . . 12

2.2 .9 Árvore . . . . . . . . . . . . . . . . . . . . . . . . . 12

2.2 .10 Floresta . . . . . . . . . . . . . . . . . . . . . . . . 12

2.2 .11 Corte . . . . . . . . . . . . . . . . . . . . . 12

2.3 Grafos a partir de imagens . . . . . . . . . . . . . . . . . . . . . . . . 12

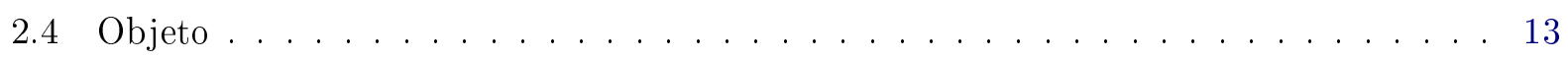

2.5 Avaliação do desempenho entre métodos de segmentação . . . . . . . . . . . . . . . 13

2.5.1 Acurácia . . . . . . . . . . . . . . . . . . . . . 13 
2.5.2 Marcadores escolhidos por erosão do gabarito . . . . . . . . . . . . . . 15

2.5.3 Marcadores escolhidos por um usuário robô . . . . . . . . . . . . . . . 17

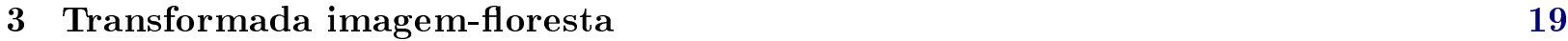

3.1 Definição . . . . . . . . . . . . . . . . . . . . . . . . . . . . 19

3.1 .1 Função de conexidade . . . . . . . . . . . . . . . . . . . . 20

3.1 .2 Caminho ótimo . . . . . . . . . . . . . . . . . . . . . 21

3.1 .3 Função de conexidade suave . . . . . . . . . . . . . . . . . . . . . 21

3.1 .4 Caminho ótimo completo . . . . . . . . . . . . . . . . . . . 22

3.2 Segmentação por competição de sementes . . . . . . . . . . . . . . . . . 22

$3.2 .1 \quad$ Algoritmo geral da IFT . . . . . . . . . . . . . . . . . . . . . . . 22

3.2 .2 Exemplo de segmentação . . . . . . . . . . . . . . . . . . . . . . 23

3.2 .3 Zonas de empate . . . . . . . . . . . . . . . . . . . . . 24

3.2 .4 Políticas de desempate . . . . . . . . . . . . . . . . . . . . 24

4 Funções de conexidade não suaves e sua classificação $\quad 29$

4.1 Definição . . . . . . . . . . . . . . . . . . . . . . . . . . . . . 29

4.2 Classificação . . . . . . . . . . . . . . . . . . . . . . . . . . . . . . . . . 29

4.3 Um primeiro exemplo de FCNS: Resolvendo empates da $f_{\max } \ldots \ldots \ldots$. . . . . . 30

4.4 Função de energia (corte ótimo) . . . . . . . . . . . . . . . . . . . . . 31

$5 \quad$ Funções de conexidade não suaves com pesos adaptativos $\quad 33$

5.1 Segmentação com funções de conexidade não suaves . . . . . . . . . . . . . . 33

5.2 Resultados experimentais para a segmentação de imagens com forte inomogeneidade 36

6 Funções de conexidade não suaves para segmentação com polaridade de borda 39

6.1 Transformada imagem-floresta orientada (OIFT) . . . . . . . . . . . . . . . 39

6.2 Segmentação pela OIFT com as funções de conexidade não suaves $f_{i, \max }^{b k g}$ e $f_{o, \max }^{b k g} \quad \ldots \quad 41$

6.2 .1 Resultados experimentais . . . . . . . . . . . . . . . . . 42

6.3 Segmentação pela OIFT com as funções de conexidade não suaves $f_{i, \omega}$ e $f_{o, \omega} \ldots \ldots$. . 45

6.3 .1 Resultados experimentais . . . . . . . . . . . . . . . . 46

7 Transformada imagem-floresta com convexidade geodésica em estrela 49

7.1 Convexidade em estrela . . . . . . . . . . . . . . . . . . . . . . . . . 49

7.2 Segmentação com restrições de convexidade geodésica em estrela . . . . . . . . . . 51

7.3 Algoritmo GSC-IFT . . . . . . . . . . . . . . . . . . . . . . . . 52

7.4 Resultados experimentais . . . . . . . . . . . . . . . . . . 54

8 Transformada imagem-floresta com tratamento simultâneo de polaridade de borda e restrições de forma

8.1 Segmentação via OIFT com restrições de conexidade geodésica em estrela através das funções $f_{i, \max }^{b k g}$ e $f_{o, \max }^{b k g} \ldots \ldots \ldots \ldots \ldots \ldots \ldots \ldots$

8.1.1 Resultados experimentais . . . . . . . . . . . . . . . . . 62 
9 Conclusões $\quad 65$

9.1 Considerações Finais . . . . . . . . . . . . . . . . . . . . . 65

9.2 Contribuições . . . . . . . . . . . . . . . . . . . . . . . . 65

9.3 Sugestões para pesquisas futuras $\ldots \ldots \ldots \ldots \ldots \ldots \ldots \ldots \ldots \ldots \ldots \ldots$

$\begin{array}{lll}\text { A Funções de conexidade não suaves } & 67\end{array}$

A.1 Função soma das arestas máximas $\left(f_{\sum \max }\right) \ldots \ldots \ldots \ldots \ldots \ldots$

A.2 Função distância mínima de barreira $\left(f_{\uparrow}\right) \ldots \ldots \ldots \ldots \ldots \ldots$

A.3 Função amplitude máxima das intensidades relativas $\left(f_{\max |\triangle I|}\right) \ldots \ldots \ldots \ldots$

A.4 Função soma do valor absoluto das intensidades relativas $\left(f_{\sum|\triangle I|}\right) \ldots \ldots . . .73$

A.5 Função de intensidade $\left(f_{I}\right) \ldots \ldots \ldots \ldots \ldots \ldots \ldots \ldots$

A.6 Função lexicográfica da soma das arestas máximas $\left(f_{\Sigma \max }^{l e x}\right) \quad \ldots \ldots \ldots \ldots \ldots$

B Prova das proposições para qualquer função $f \in\left(C_{1} \cap C_{4}\right) \backslash C_{2} \quad 79$

C Prova dos teoremas de otimalidade dos cortes internos/externos na OIFT $\quad 81$

$\begin{array}{ll}\text { D Prova de otimalidade do algoritmo GSC-IFT } & 87\end{array}$

E Prova de otimalidade do método GSC-OIFT $\quad 89$

$\begin{array}{ll}\text { Referências Bibliográficas } & 91\end{array}$

$\begin{array}{ll}\text { Índice Remissivo } & 98\end{array}$ 


\section{Lista de Abreviaturas}

AFC Conexidade fuzzy absoluta (Absolute Fuzzy Connectedness).

BIA Software analisador de imagem cerebral (Brain Image Analyzer).

CInApCe Cooperação Interinstitucional de Apoio a Pesquisas sobre o Cérebro.

DC Distance Cut.

FCNS Funções de conexidade não suaves.

GGC Corte generalizado de grafo (Generalized Graph Cut).

GSC Convexidade geodésica em estrela (Geodesic Star Convexity).

GSC-IFT IFT com convexidade geodésica em estrela (IFT with Geodesic Star Convexity).

GSC-OIFT OIFT com convexidade geodésica em estrela (OIFT with Geodesic Star Convexity).

IFT Transformada imagem-floresta (Image Foresting Transform).

IFT-SC IFT por competição de sementes ( IFT Segmentation by Seed Competition).

IRFC Conexidade fuzzy relativa iterativa (Iterative Relative Fuzzy Connectedness).

MBD Distância mínima de barreira (Minimum Barrier Distance).

MF Min-cut/max-flow.

OIFT Transformada imagem-floresta orientada (Oriented Image Foresting Transform).

PW Power Watersheds.

RFC Conexidade fuzzy relativa (Relative Fuzzy Connectedness).

RM Ressonância magnética.

RW Passeios aleatórios (Random Walks).

TC Tomografia computadorizada.

THR Thresholding. 


\section{Lista de Símbolos}

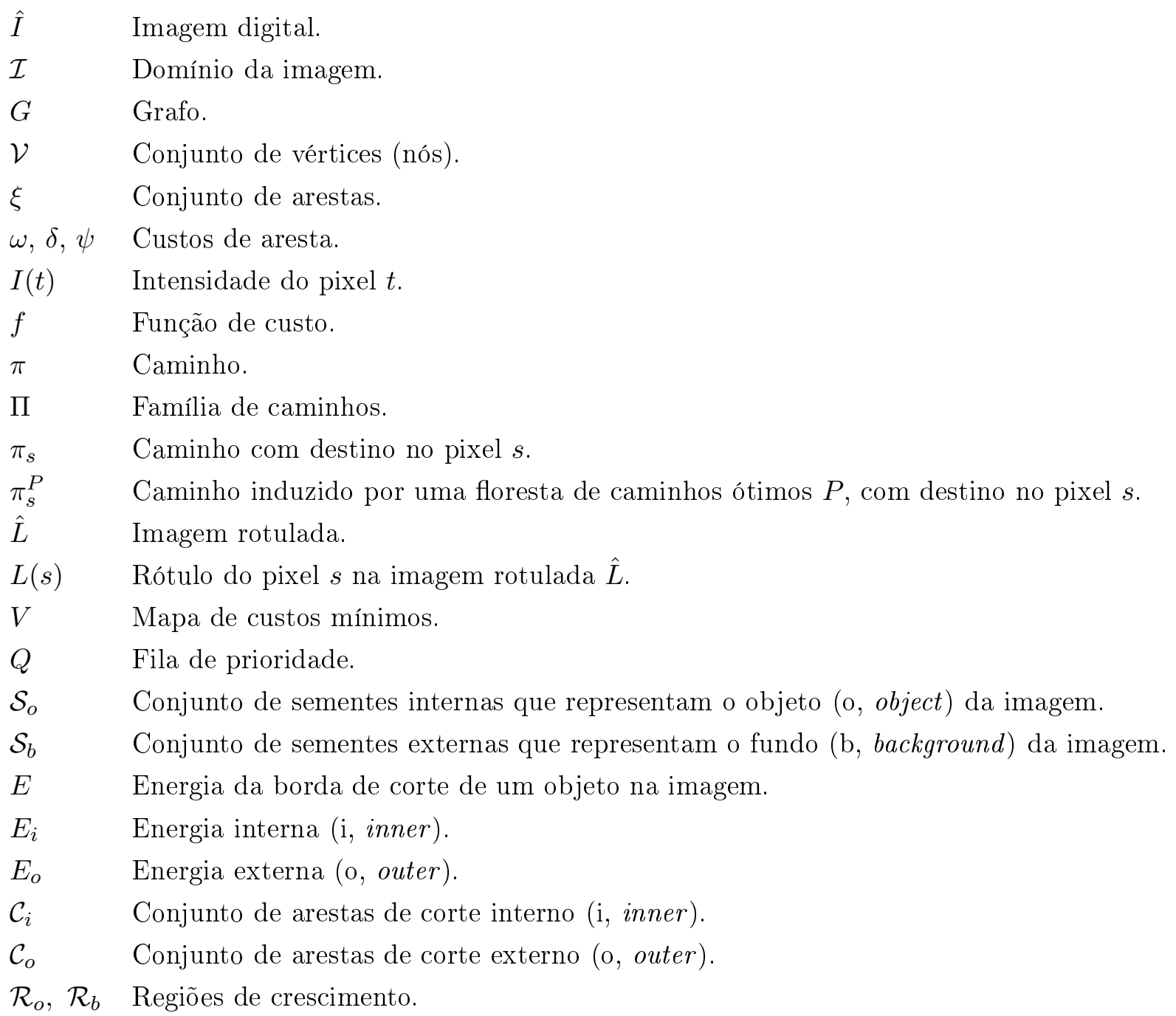




\section{Lista de Figuras}

1.1 Representação esquemática das relações entre os métodos (RW - random walks, MF - min-cut/max-flow, DC - distance cut, IFT - image foresting transform, THR - thresholding, IRFC - iterative relative-fuzzy connectedness, RFC - relative-fuzzy connectedness, AFC - absolute-fuzzy connectedness, PW - power watersheds). Diagrama extraído de [Miranda e Falcão (2011)].

1.2 (a) Marcadores de realce selecionados pelo usuário. (b) Mapa de pertinência do objeto obtido por meio da classificação supervisionada "fuzzy". (c) Imagem dos pesos das arestas, mostrando o realce obtido na borda do objeto. Note que as transições internas entre as listras foram enfraquecidas. (d) A extração final de uma única zebra é obtida pela IFT diferencial, a partir de um novo conjunto de marcadores, explorando a conexidade ótima no espaço da imagem. . . . . . . . . . . . . . . . . 4

1.3 Imagens em que o objeto e o fundo têm cores muito semelhantes. . . . . . . . . . . 5 5

1.4 Exemplo de inomogeneidade de campo. (a) As marcas amarelas $+/ \oplus$ indicam regiões correspondentes em diferentes tecidos dos hemisférios. À esquerda, as substâncias cinzenta e branca apresentam valores de intensidade de 57 e 145, respectivamente, para os pontos marcados. Já na região direita da imagem, temos valores consideravelmente maiores, 109 e 221. (a-c) Variando as configurações de brilho e contraste é possível melhor observar o problema. (d-f) Variações de brilho e contraste em uma fatia axial, revelando a inomogeneidade. . . . . . . . . . . . . . . . .

1.5 Problemas resultantes da inomogeneidade em imagens de ressonância magnética de 3 Tesla que geram erros em técnicas automáticas padrões (Brain Extraction Tool (BET) [Smith (2002)] e Clouds [Miranda et al. (2008b, 2009)] respectivamente). . . 6

2.1 Adjacências Euclideanas: caso $2 D$ com raio (a) $\rho=1$, (b) $\rho=\sqrt{2}$, (c) $\rho=\sqrt{5}$, e caso

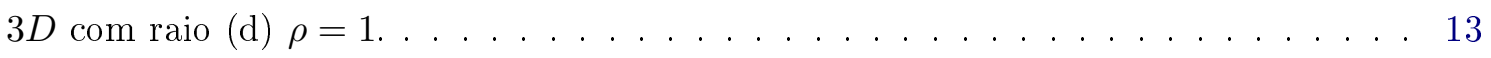

2.2 Exemplo de segmentação para um método específico . . . . . . . . . . . . . . . . 14

2.3 Matriz de confusão. . . . . . . . . . . . . . . . . . . . . . . 15

2.4 Exemplo de marcadores escolhidos por erosão do gabarito . . . . . . . . . . . . 16

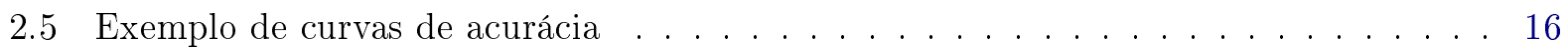

2.6 Exemplo de marcadores escolhidos por um usuário robô para a segmentação interativa 18

3.1 (a) Extensão de um caminho $\pi_{s}$ pela aresta (s,t). (b) Grafo de vizinhança-4 mostra um caminho $\pi_{t}$ (linha pontilhada) representado no sentido inverso onde $\mathrm{P}(\mathrm{t})$ é o nó predecessor de $t$ e $R\left(\pi_{t}\right)$ é o pixel raiz/origem do caminho $\pi_{t}$ (c) Floresta de espalhamento $P$ com dois nós raízes, $r_{1}$ e $r_{2} \ldots \ldots \ldots \ldots \ldots$ 
3.2 Exemplo de segmentação com dois marcadores, o marcador $\mathcal{S}_{o}$ interno representa o objeto e o marcador $\mathcal{S}_{b}$ o fundo. Em (a) são mostrados caminhos ótimos para pixels internos, como o caminho de $\mathcal{S}_{o}$ até $a$, e pixels externos, como o caminho de $\mathcal{S}_{b}$ até $b$. As linhas pontilhadas representam caminhos não ótimos. Em (b) é mostrada a borda entre as florestas ótimas interna e externa. . . . . . . . . . . . . . . . . . 24

3.3 Exemplo de segmentação pela IFT com a função suave $f_{\max } \ldots \ldots \ldots$. . . . . . 25

3.4 Um grafo de vizinhança-4 com duas zonas de empate . . . . . . . . . . . . . . . 26

3.5 Exemplos de políticas de desempate FIFO e LIFO para um grafo vizinhança-4 e um

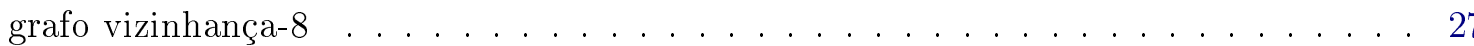

4.1 Representação esquemática das relações entre funções de conexidade suaves e não suaves: $C_{1}, C_{2}, C_{3}$, e $C_{4}$ são conjuntos de funções de conexidade que satisfazem suas respectivas condições. O conjunto $M I$ representa as funções monotonicamente incrementais. . . . . . . . . . . . . . . . . . . . 30

4.2 Contra-exemplo da função de custo $f_{\max }^{b k g}$ para a condição de suavidade $C 2 \ldots 31$

4.3 (a) Representação esquemática de três possíveis bordas de corte, alguns valores de aresta representativos $\omega(s, t)$ são representados para cada região de segmento de borda. (b) Resultado da segmentação usando a função $f_{\text {max }}$ para as sementes mostradas. O segmento de borda com peso de aresta $\omega(s, t)$ igual a 214 tem preferência sobre os segmentos com pesos 124 e 93, devido à maximização da energia $E$ (Equação 4.3) dada pelo mínimo peso na borda de corte.

4.4 (a) Representação esquemática de um grafo onde só alguns valores de aresta representativos $\omega(s, t)$ são representados para cada região e segmento de borda. Os valores ótimos de conexidade para $f_{\max }$ (Equação 3.6) dados por $V(a)=1, V(b)=4$, e $V(c)=2$ são também indicados. O segmento de borda com pesos de aresta $\omega(s, t)$ iguais a 5 tem preferência sobre o segmento com peso 4 , devido à propriedade de otimização por partes. Note que ambas soluções levam ao mesmo corte máximo com valor mínimo $E=3 \ldots \ldots \ldots \ldots \ldots \ldots \ldots \ldots \ldots$

5.1 (a) Um grafo $G$ da imagem $\mathcal{I}$ com adjacência 4-conexo e sementes $\mathcal{S}=\left\{s_{1}, s_{2}\right\}$, no qual os números nas arestas representam os pesos. (b) Floresta calculada pela IFT com a função não suave $f_{\Sigma \max }^{\text {lex }} \in\left(C_{1} \cap C_{4}\right) \backslash C_{2}$. Os números dentro dos nós indicam os valores do mapa $V$ de custos com duas componentes em ordem lexicográfica. Nessa figura as setas indicam o mapa de predecessores. (c) Note que o caminho $\pi_{a}$ com destino no pixel $a$ a partir da semente $s_{1}$ é um caminho ótimo completo, mas o caminho $\pi_{b}$ com destino no nó $b$ a partir da mesma semente não é ótimo, dado que existe outro caminho $\pi_{b}^{\prime}$ (seguindo a linha tracejada) até ele a partir da semente $s_{2}$ que oferece um melhor custo, (isto é, $f_{\Sigma \max }^{\text {lex }}\left(\pi_{b}^{\prime}\right)=(11 ; 3)<f_{\Sigma \max }^{\text {lex }}\left(\pi_{b}\right)=(12 ; 5)$ ). Isso mostra que a função $f_{\Sigma \max }^{l e x}$ é não suave. (d) Aplicando a Proposição 1 temos uma floresta calculada para o conjunto $\mathcal{O}^{1}$, composta por caminhos ótimos no grafo $G$ (primeira otimização). (e) Temos o grafo residual $G^{2}$, o qual será usado em uma segunda otimização. (f) Floresta ótima calculada a partir do grafo $G^{2}$, terminando o processo com $\mathcal{O}^{1} \cup \mathcal{O}^{2}=\mathcal{I}$ 
5.2 Resultados usando um usuário robô para a segmentação do conjunto de dados do

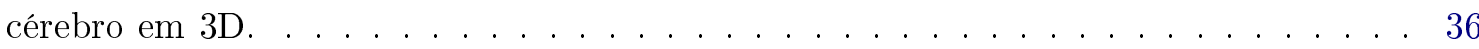

5.3 Resultado da segmentação 3D do cérebro para os mesmos marcadores selecionados pelo usuário para as funções: (a)-(d) $f_{\max }$, e (e)-(h) $f_{\sum|\triangle I|}^{\text {lex }} \ldots \ldots \ldots \ldots$. . . . . 37

5.4 Segmentação 3D da substância branca para os mesmos marcadores selecionados pelo usuário. Resultados obtidos pelas funções (a)-(d) $f_{\max }$ sobre um gradiente realçado,

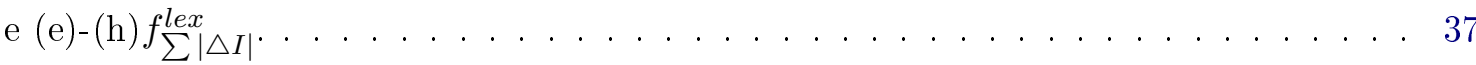

5.5 Exemplo de realce de bordas em uma imagem com problemas de inomogeneidade. Os marcadores amarelos, representando o objeto, indicam a substância branca e os marcadores brancos representam o fundo. Usando as técnicas de realce das bordas do objeto apresentadas em [Miranda et al. (2010)], obtemos os mapas de pertinência (c) e (d), para marcadores localizados no hemisfério (a) esquerdo e (b) direito, respectivamente.

6.1 Exemplo de segmentação baseada em borda, onde a propriedade de orientação de borda do live wire o ajuda a distinguir entre dois segmentos de borda similares [Falcão et al. (2000)].

6.2 Em (a) considera-se uma energia interna $E_{i}$ para os cortes internos na borda do objeto. Em (b) uma energia externa $E_{o}$ é considerada para os cortes externos na mesma borda. . . . . . . . . . . . . . . . . . . . . . 40

6.3 Em (a) temos uma transição de pixels de escuro para claro na borda do objeto com cortes internos $(p, q) \in \xi$. Em (b) temos uma borda com transições de pixels de claro pra escuro considerando cortes externos $(p, q) \in \xi \ldots \ldots \ldots \ldots \ldots$

6.4 A função $f_{o \text {,max }}^{b k g}$ faz uso de arestas invertidas para caminhos a partir de $\mathcal{S}_{b}$. Suponhamos que $\mathcal{S}_{b}$ conquista a região branca da esquerda com custo 0 , dado que seus pixels são interconectados por arestas com peso 0 . Os pixels dentro da região cinza tem também arestas com peso 0 , assim $\mathcal{S}_{o}$ fornece custos melhores a eles $(2 \times 0+1=1$ contra $2 \times 1=2$ oferecido por $\mathcal{S}_{b}$ ). O melhor custo oferecido por $\mathcal{S}_{o}$ ao nó $b$ é $2 \times 8+1=17$, enquanto que $\mathcal{S}_{b}$ poderia ter fornecido um melhor custo a $b(2 \times 1=2)$, mas $b$ é atribuído ao objeto. Portanto, a função não é suave.

6.5 (a) Gabarito da segmentação do osso tálus em uma fatia de imagem RM do pé. (b) Exemplo de conjuntos de sementes obtidos pela erosão do gabarito de segmentação. (c) Resultado da segmentação por $f_{\max }^{b k g}$. (d) Um resultado melhor de segmentação é obtido mediante o uso da informação da orientação de borda do objeto por meio de $f_{i, \max }^{b k g}$

6.6 (a) Gabarito da segmentação do osso calcâneo em uma imagem RM do pé. (b) Exemplo de conjuntos semente obtidos pela erosão do gabarito. (c) Resultado da segmentação por $f_{\max }^{b k g}$. (d) Um resultado melhor de segmentação é obtido explorando a orientação de borda usando $f_{i, \max }^{b k g} \ldots \ldots \ldots \ldots \ldots \ldots$

6.7 (a) Gabarito da segmentação da coluna vertebral em uma imagem TC. (b) Exemplo de conjuntos semente obtidos erodindo o gabarito da segmentação. (c) Resultado da segmentação por $f_{\max }^{b k g}$. (d) Um resultado melhor da segmentação é obtido explorando a orientação de borda usando $f_{o, \max }^{b k g} \ldots \ldots \ldots \ldots \ldots \ldots \ldots$ 
6.8 Curvas médias de acurácia para $f_{\max }^{b k g}\left(\right.$ normal), $f_{i, \max }^{b k g}\left(\right.$ inner-cut), $f_{o, \max }^{b k g}$ (outer-cut) para a segmentação do (a) tálus, (b) calcâneo, (c) coluna vertebral, e (d) fígado. . . . 45

6.9 Curvas médias de acurácia para $f_{\max }^{b k g}\left(\right.$ normal), $f_{i, \max }^{b k g}\left(\right.$ inner-cut), $f_{o, \max }^{b k g}$ (outer-cut) para a segmentação 3D do cerebelo. . . . . . . . . . . . . . . . . . . . . . 45

6.10 (a) Imagem de entrada com transições igualmente ponderadas, tendo a mesma orientação. (b) Resultado da OIFT usando $f_{o, \max }^{b k g}$ como proposto em [Miranda e Mansilla (2014)], atribuindo regiões ambíguas para o fundo. (c) OIFT usando $f_{o, \omega}$ com uma política de desempate FIFO fornece partições mais igualmente balanceadas. . . . . . 47

6.11 Curvas médias de acurácia (Dice) usando o gradiente Sobel para a segmentação 3D dos ossos: (a) tálus, e (b) calcâneo. . . . . . . . . . . . . . . . . . . . . . . . . . . . . 47

6.12 Curvas médias de acurácia (Dice) usando o valor absoluto da diferença de intensidades para a segmentação 3D dos ossos: (a) tálus, e (b) calcâneo. . . . . . . . . . . . . 48

6.13 Curvas médias de acurácia para diferentes métodos usando imagens coloridas. . . . . 48

7.1 Em (a) consideramos uma imagem RM do cérebro (objeto de forma regular). Em (b) é mostrado o gradiente da imagem. Em (c) temos o resultado da segmentação do cérebro pela IFT-SC com $f_{\max }$ que apresenta uma forma não regular. . . . . . . . . . 49

7.2 Convexidade em estrela: Em (a) é apresentado um objeto convexo em estrela, o qual não é convexo como mostrado em (b). Um objeto que viola a restrição de convexidade em estrela é mostrado em (c), onde $p$ é um ponto do objeto dado, e $c$ é o centro de estrela considerado.

7.3 Considere um objeto $\mathcal{O}$ (área sombreada), com o conjunto de centros de estrela $C=\{a, b\} . \operatorname{Em}(\mathrm{a})$ temos o argumento de visibilidade: Qualquer ponto $p \in \mathcal{O}$ deve ser visível ao menos por um dos centros de estrela (por exemplo, $p$ é visível através de $\overline{a p}$, embora este não seja visível através de $\overline{b p}$ ). Em (b) temos o argumento da distancia mais curta: Cada ponto é analisado exclusivamente em relação a seu centro mais próximo (por exemplo, $q$ está mais próximo de $a$, e $p$ mais próximo de $b$ ). Em (c) temos uma versão discreta pelo caminho mais curto no grafo de imagem. . . . . . 50

7.4 Efeito do parâmetro de potência $\beta$ sobre a topologia da floresta: A floresta de caminhos ótimos de $f_{\text {sum }}$ para (a) $\beta=0.0$, (b) $\beta=0.3$, (c) $\beta=0.4$, e (d) $\beta=0.5$. . . . 51

7.5 Efeitos do parâmetro de potência $\beta$ sobre a topologia da floresta: Em (a) é mostrada a floresta de caminhos ótimos de $f_{\text {sum }}$ para $\beta=0$. Em (b) é mostrada a segmentação resultante pelo algoritmo GSC-IFT (Algoritmo 2). Resultados similares são mostrados em (c) e (d) para $\beta=0.3$, e em (e) e (f) para $\beta=0.5$. . . . . . . . . .

7.6 (a) Imagem sintética com três possíveis bordas de corte, onde $s_{o}$ e $s_{b}$ são sementes do objeto e fundo respectivamente, e a floresta $P_{\text {sum }}$ permite a visibilidade das restrições de forma. (b) Após o pixel $a$ sair da fila $Q$ com rótulo 1 , temos a violação da visibilidade do pixel $a$ em relação a sua semente mais próxima $s_{o}$. (c) O procedimento Conquistar_Caminho atribui rótulo 1 a todos seus predecessores, permitindo a expansão dos caminhos do objeto na região cinza claro. (d) O pixel $b$ sai da fila $Q$ com rótulo de fundo, bloqueando a visibilidade dos seus descendentes em $P_{\text {sum }}$ em relação a $s_{o}$ (e) O procedimento Podar_Árvore atribui rótulo 0 a seus descendentes, permitindo a expansão dos caminhos de fundo na região cinza escuro. (f) Resultado da segmentação (linha amarela pontilhada) com o algoritmo GSC-IFT. . . . . . . . . 55 
7.7 (a) Curvas de acurácia dos métodos GSC-IFT, IRFC e PW $(q=2)$ para a segmentação de: (a) calcâneo, (b) tálus, (c) fígado, e (d) mama. . . . . . . . . . . . . . 56

7.8 Para cada imagem individual, os métodos podem ser ranqueados de acordo a seus valores médios do coeficiente de Dice, como primeiro (o melhor), segundo, ou terceiro (o pior). Calculando a frequência para cada posição de classificação, temos uma distribuição de classificação para segmentar: (a) calcâneo, (b) tálus, (c) fígado, e (d)

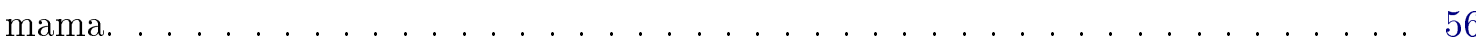

7.9 Segmentação do osso tálus: Em (a) temos o resultado do IRFC para os marcadores selecionados. Em (b) temos que um melhor resultado pode ser obtido por GSC-IFT para os mesmos marcadores. . . . . . . . . . . . . . . . . . . 57

7.10 Exemplo de segmentação do cérebro (skull stripping) em 3D usando marcadores selecionados pelo usuário. Em (a) e (b) temos o resultado da segmentação por IFTSC. Em (c) e (d) temos que um resultado melhor é obtido pela GSC-IFT com $\beta=0.1 .57$

7.11 Em (a),(d) e (g) temos imagens de entrada com marcadores selecionados pelo usuário. Em (b), (e) e (h) temos o resultado da segmentação pela IFT-SC. Em (c), (f) e (i) temos o resultado da segmentação pelo GSC-IFT $(\beta=0.1) \ldots \ldots \ldots \ldots$. . . . 58

8.1 (a) Uma imagem original com marcadores $S_{o}$ e $S_{b}$. (b) Resultado da segmentação com IFT. (c) Resultado da segmentação com OIFT. (d) Resultado da segmentação com GSC-IFT. (e) Resultado da segmentação considerando simultaneamente a polaridade de borda e as restrições de forma $($ GSC-OIFT) $\ldots \ldots \ldots$. . . . . . . . . . . 60

8.2 Imagem rotulada $L$ com rótulo $L(s)=1$ se o pixel $s$ pertence ao objeto e rótulo $L(s)=0$ se pertence ao fundo. Em (a) é mostrada a violação da restrição de convexidade geodésica em estrela quando existe uma aresta $(s, t) \in\left(\mathcal{C}_{i}(L) \cap \xi_{P_{\text {sum }}}^{i}\right)$. Em (b) é mostrada a violação da restrição de convexidade geodésica em estrela quando existe uma aresta $(s, t) \in\left(\mathcal{C}_{o}(L) \cap \xi_{P_{\text {sum }}}^{o}\right)$.

8.3 (a) Imagem sintética com três possíveis bordas de corte, com mesma orientação (claro para escuro) floresta $P_{\text {sum }}$ e semente interna $s_{o}$ (centro de estrela). As arestas com valor $-\infty$ representam arestas $(s, t) \in \xi_{P_{\text {sum }}}^{o}$ que violam as restrições de forma pela GSC. As setas azuis representam cortes externos nas bordas e as vermelhas (setas pontilhadas) cortes internos. (b) Pixels são conquistados pela semente interna $s_{o}$. (c) Pixels são conquistados pela semente externa $s_{b}$. (d) Resultado da segmentação (linha amarela) pela GSC-OIFT com $f_{o, \max }^{b k g} \ldots \ldots \ldots \ldots \ldots \ldots \ldots$

8.4 (a) Curvas médias de acurácia para a segmentação do fígado para diferentes valores de $\beta$ : (a) $\beta=0.0$, (b) $\beta=0.2$, (c) $\beta=0.5$, e (d) $\beta=0.7 \ldots \ldots \ldots \ldots$. . . . . 63

8.5 Resultados para marcadores selecionados pelo usuário: (a) IRFC (IFT com $f_{\max }$ ), (b) OIFT $\left(f_{o, \max }^{b k g} \operatorname{com} \alpha=0.5\right)$, (c) GSC-IFT $(\beta=0.7, \alpha=0.0)$, e (d) GSC-OIFT $(\beta=0.7, \alpha=0.5)$.

8.6 Exemplo de skull stripping 3D em RM: (a) IRFC (IFT com $f_{\max }$ ), (b) GSC-IFT $(\beta=0.3, \alpha=0.0)$, e (c) GSC-OIFT $(\beta=0.3, \alpha=0.5)$, para os mesmos marcadores selecionados pelo usuário. . . . . . . . . . . . . . . . . 64 
A.1 Contra exemplo da segunda e terceira condições $(C 2$ e $C 3)$ para a função $f_{\Sigma \max }$, onde $\pi_{t}=\pi_{s} \cdot\langle s, t\rangle$ é um caminho ótimo e $\pi_{t}^{\prime}=\pi_{s}^{\prime} \cdot\langle s, t\rangle$ é um caminho não ótimo, a partir das sementes $S_{1}$ e $S_{2}$ respectivamente.

A.2 Contra exemplo da condição $C 4$ para a função $f_{\Sigma \max }$, onde $\pi_{t}=\pi_{s} \cdot\langle s, t\rangle, \pi_{s}$, e $\pi_{s}^{\prime}$ são caminhos ótimos, $\pi_{t}^{\prime}=\pi_{s}^{\prime} \cdot\langle s, t\rangle$ é um caminho não ótimo, e $S_{1}, S_{2}$ são sementes. 69

A.3 Contra exemplo para a segunda e terceira condições $(C 2$ e $C 3)$ para a função $f_{\uparrow}$, onde $\pi_{t}=\pi_{s} \cdot\langle s, t\rangle$ é um caminho ótimo, e $\pi_{t}^{\prime}=\pi_{s}^{\prime} \cdot\langle s, t\rangle$ é um caminho ótimo, a

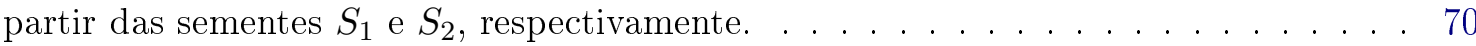

A.4 Contra exemplo da condição $C 4$ para a função $f_{\uparrow}$, onde $\pi_{t}=\pi_{s} \cdot\langle s, t\rangle, \pi_{s}$, e $\pi_{s}^{\prime}$ são caminhos ótimos; $\pi_{t}^{\prime}=\pi_{s}^{\prime} \cdot\langle s, t\rangle$ é um caminho não ótimo, e $S_{1}, S_{2}$ são sementes. . . 71

A.5 Contra exemplo da segunda e terceira condições $(C 2$ e $C 3)$ para as funções $f_{\max |\triangle I|}$ e $f_{\Sigma|\triangle I|}$. O caminho $\pi_{t}=\pi_{s} \cdot\langle s, t\rangle$ é um caminho ótimo, e $\pi_{t}^{\prime}=\pi_{s}^{\prime} \cdot\langle s, t\rangle$ não é um caminho ótimo, a partir das sementes $S_{1}$ e $S_{2}$, respectivamente.

A.6 Contra exemplo da condição $C 4$ para as funções $f_{\max |\triangle I|}$ e $f_{\Sigma|\triangle I|}$. Os caminhos $\pi_{t}=\pi_{s} \cdot\langle s, t\rangle, \pi_{s}$, e $\pi_{s}^{\prime}$ são ótimos; $\pi_{t}^{\prime}=\pi_{s}^{\prime} \cdot\langle s, t\rangle$ é um caminho não ótimo, e $S_{1}$ e

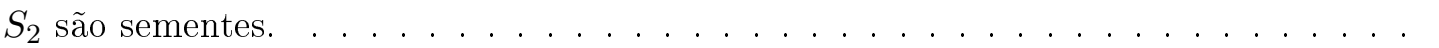

A.7 Contra exemplo da primeira condição $(C 1)$ para a função $f_{I}$, onde $\pi_{t}=\pi_{s} \cdot\langle s, t\rangle$ é um caminho ótimo a partir da semente $S_{1} \ldots \ldots \ldots \ldots \ldots \ldots$

A.8 Contra exemplo da segunda e terceira condições $(C 2$ e $C 3)$ para a função $f_{\Sigma \max }^{\text {lex }}$, onde $\pi_{t}=\pi_{s} \cdot\langle s, t\rangle$ é um caminho ótimo, e $\pi_{t}^{\prime}=\pi_{s}^{\prime} \cdot\langle s, t\rangle$ é um outro caminho não ótimo, a partir das sementes $S_{1}$ e $S_{2}$ respectivamente. . . . . . . . . . . . 76

C.1 São mostrados dois cenários de bordas de corte: (a) Duas bordas candidatas são mostradas com pesos mínimos apontando para o exterior, dados por $\omega\left(u_{1}, v_{1}\right)$ e $\omega\left(u_{2}, v_{2}\right)$ respectivamente. (b) Duas possíveis bordas de corte ótimas são mostradas, ambas com valor ótimo na Equação 6.2 dado por $\omega(a, b)$. As linhas pontilhadas representam as partes da borda em questão, $\mathcal{X}_{1}$ e $\mathcal{X}_{2}$, com pesos mínimos apontando para o exterior, dados por $\omega\left(u_{1}, v_{1}\right)$ e $\omega\left(u_{2}, v_{2}\right)$ respectivamente. . . . . . . . 83 


\section{Lista de Tabelas}

6.1 Descrição dos métodos usados nos experimentos da segmentação pela OIFT $\operatorname{com} f_{i, \omega}$

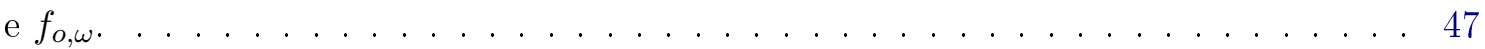

9.1 Relação dos métodos propostos e o tipo de imagem a ser segmentada. . . . . . . . . . 66 
xxii LISTA DE TABELAS 


\section{Capítulo 1}

\section{Introdução}

Segmentar uma imagem consiste em particioná-la em regiões relevantes para uma dada aplicação. Comumente, objetos de interesse devem ser isolados dentro do domínio da imagem, a fim de permitir a obtenção de informações significativas para uma determinada aplicação. Como consequência, a segmentação da imagem tem muitas aplicações práticas, incluindo: percepção autônoma de máquina, reconhecimento de caracteres, inspeção industrial automática e sistemas de controle de tráfego [Gonzalez e Woods (1992)]. Em publicidade, a segmentação de imagens naturais é usada na edição de fotos e vídeo, tal como para extrair objetos de primeiro plano de uma imagem e compô-los em um novo fundo [Bai e Sapiro (2007)].

Em imagens médicas, a grande variedade de tecnologias de imagem (por exemplo, tomografia computadorizada, tomografia por emissão de pósitrons, angiografia por ressonância magnética, ressonância magnética, ressonância magnética funcional, SPECT, ultra-som) promoveu a prática da segmentação de imagem na medicina [Bueno et al. (2001); Falcão et al. (1998); Li et al. (2006); Suetens (2009); Toennies (2012)]. A análise quantitativa de estruturas cerebrais, isoladas por técnicas de segmentação, a partir de imagens de Ressonância Magnética (RM) tem desempenhado um papel importante para a pesquisa em neurologia. Medidas de volume, textura e assimetria de forma/textura podem ser utilizadas para relacionar alterações morfológicas com diversas doenças, sendo útil nos seus diagnósticos e tratamentos [Bonilha (2004); Castellano et al. (2004); Natsume e et al. (2003); Sandock et al. (2000); Suetens (2009); Toennies (2012)].

A segmentação de imagem é um dos problemas mais fundamentais e desafiadores em processamento de imagem e visão computacional. Segmentação manual de imagens [Bonilha (2004); Bonilha et al. (2003)] é uma tarefa extremamente demorada e tediosa, sensível a subjetividade do operador e sujeita a falhas em função do cansaço/fadiga. Por outro lado, técnicas de segmentação automática geralmente tendem a apresentar algum tipo de erro, podendo até mesmo falhar em circunstâncias críticas. O conhecimento de alto nível do usuário, sobre o domínio específico da aplicação, é muitas vezes necessário em análise de imagens médicas devido à presença de estruturas com bordas mal definidas, e na segmentação de imagens naturais, devido à sua natureza heterogênea. Como consequência, resultados cuidadosos e precisos de segmentação comumente requerem considerável assistência do usuário [Beucher e Meyer (1993); Bueno et al. (2001); Falcão e Bergo (2004); Falcão et al. (2000); Kass et al. (1987); Protiere e Sapiro (2007)].

Matemática Discreta fornece uma estrutura elegante para o processamento de imagens, sendo rica em algoritmos eficientes, com provas de corretude. Como consequência, muitos métodos de segmentação de imagem foram modelados como problemas de busca e otimização em gra- 
fos [Couprie et al. (2010); Falcão et al. (1998, 2000); Grady (2006); Lézoray e Grady (2012); Peng et al. (2011); W.Yang et al. (2010)]. Em tais abordagens, um grafo derivado da imagem é calculado e o usuário indica restrições fortes, selecionando alguns dos seus vértices como sementes (ou pontos de ancoragem de contorno) para o reconhecimento, enquanto que o delineamento subsequente é realizado pelo computador em tempo interativo. O delineamento é calculado e exibido através de uma partição ótima do grafo satisfazendo o conjunto fornecido de restrições. Correções podem então ser realizadas pela adição de novas sementes e/ou a remoção de sementes. Estes métodos de segmentação em grafos podem ser classificados como sendo baseados em borda ou região, de acordo com a estratégia de representação usada para segmentar os objetos.

Métodos por região são, em geral, preferíveis aos métodos por borda devido a sua facilidade de extensão para imagens multidimensionais. Vários métodos de segmentação por região podem ser derivados a partir de diferentes arcabouços principais, tais como bacia hidrográfica (watershed) [Cousty et al. (2010); Roerdink e Meijster (2000); Vincent e Soille (1991)], passeios aleatórios (random walks) [Grady (2006)], conexidade "fuzzy" (fuzzy connectedness) [Ciesielski et al. (2007); Udupa et al. (2002)], transformada imagem-floresta (IF T, image foresting transform) [Falcão e Bergo (2004); Falcão et al. (2004)], e corte em grafo (graph cuts) [Boykov e Funka-Lea (2006); Boykov e Jolly (2001)]. Estes arcabouços foram projetados de acordo com diferentes critérios e funções objetivo para partição do grafo.

As relações teóricas entre essas diferentes abordagens, em vista do grafo base comum, foram alvo de estudo em vários trabalhos recentes [Allène et al. (2010); Audigier e Lotufo (2007); Ciesielski e Udupa (2011); Ciesielski et al. (2011, 2012a); Couprie et al. (2010); Cousty et al. (2010); Miranda e Falcão (2009, 2011)]. A Figura 1.1 mostra um diagrama dessas relações conforme proposto por Miranda e Falcão [Miranda e Falcão (2011)], onde cada método é representado por um conjunto, e interseções/sobreposições indicam que, para qualquer imagem, os métodos podem produzir sempre o mesmo resultado de segmentação para alguma seleção apropriada dos seus parâmetros.

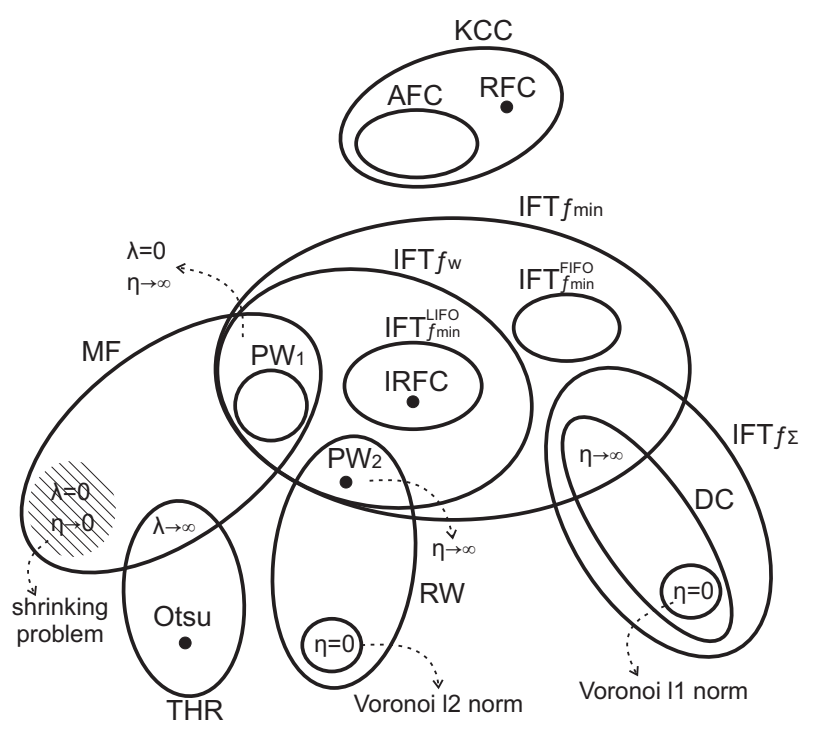

Figura 1.1: Representação esquemática das relações entre os métodos ( $R W$ - random walks, MF - mincut/max-flow, DC - distance cut, IFT - image foresting transform, THR - thresholding, IRFC - iterative relative-fuzzy connectedness, $R F C$ - relative-fuzzy connectedness, AFC - absolute-fuzzy connectedness, $P W$ power watersheds). Diagrama extraído de [Miranda e Falcão (2011)]. 
No entanto, esses métodos na maioria dos trabalhos se restringem a grafos não direcionados (algumas exceções são apresentadas nos artigos [Boykov e Funka-Lea (2006)] e [Singaraju et al. (2008)]), também a qualidade dos seus resultados de segmentação, com mínima intervenção do usuário, depende fortemente de uma estimativa adequada dos pesos atribuídos às arestas do grafo [Miranda et al. (2010)], e os métodos computacionalmente mais eficientes carecem de restrições de regularização de borda [Ciesielski et al. (2007); Cousty et al. (2010); Falcão et al. (2004)].

Nesse trabalho apresentamos soluções para esses problemas no arcabouço da transformada imagem-floresta (IFT) [Falcão et al. (2004)] através de funções de conexidade não suaves (FCNS). As funções não suaves compõem uma classe de funções de conexidade menos restrita, possibilitando configurações mais adaptativas para lidar com os problemas de inomogeneidade, polaridade de borda, e restrições de forma, conforme indicam os nossos estudos.

Historicamente, funções de conexidade não suaves foram evitadas, dado que os caminhos gerados pela IFT com FCNS podem não ter otimalidade garantida de acordo com a definição apresentada em [Falcão et al. (2004)]. Estudos anteriores consideraram apenas o uso de FCNS, como $f_{\text {euc }}$ (Equação 3.5 com adjacência 8-conexo), para calcular uma aproximação da distância Euclideana [Falcão et al. (2002); Torres et al. (2004)], embora alguns outros estudos por Herman et al. têm sugerido algumas outras vantagens práticas das FCNS no contexto da segmentação de imagens [Herman e Carvalho (2001); Miranda et al. (2008a)]. Observamos também que as funções de conexidade usadas para reconstrução superior local [Falcão (2009)] não são suaves. Mais recentemente Strand et al. propuseram o método por Minimum Barrier Distance (MBD) [Strand et al. (2013)], o qual, em cenários digitais, leva a uma FCNS; que tem mostrado bons resultados em comparações empíricas. Também, em outros trabalhos recentes, foi provado que algumas FCNS levam a resultados ótimos de acordo com outros critérios de otimalidade, como uma função de energia para perseguição ótima de bordas [Miranda et al. (2011, 2012)]. Isso reacendeu as discussões sobre a pesquisa envolvendo FCNS.

A seguir, introduzimos os três problemas principais que serão abordados nesta dissertação: $\mathrm{O}$ uso de pesos adaptativos, a incorporação da polaridade de borda via dígrafos, e a eliminação de falsos delineamentos por restrições de forma.

\section{$1.1 \quad$ Pesos adaptativos}

Nos métodos tradicionais de segmentação [Beucher e Meyer (1993); Couprie et al. (2010); Kass et al. (1987); Shi e Malik (2000); Wang e Siskind (2001)], a estimativa de peso dos arcos é geralmente tratada como um simples processo incorporado, desconsiderando, em muitos casos, as intervenções do usuário. Já em outros trabalhos [Saha e Udupa (2003); Saha et al. (2000)] uma estimativa mais sofisticada é empregada de forma integrada, porém elevando consideravelmente a complexidade dos métodos, com impactos no desempenho e/ou dificultando o seu entendimento. Mais recentemente, atenção especial passou a ser dada ao processo de estimativa de pesos, com artigos especialmente dedicados a esse tópico [Ciesielski e Udupa (2009); Miranda et al. (2010)], além de métodos com pesos mais bem elaborados [Boykov e Jolly (2001); Protiere e Sapiro (2007); Rother et al. (2004); Spina et al. (2009)].

Alguns trabalhos consideram a estimativa dos pesos como uma etapa de realce, enquanto que a partição ótima do grafo é dada em uma etapa de extração [Miranda et al. (2010); Spina et al. 
(2009)]. Informações de objeto extraídas dos marcadores (por exemplo, padrão de intensidade, cor) são cruciais para a melhoria da qualidade da estimativa dos pesos das arestas [Boykov e Jolly (2001); Miranda et al. (2010); Protiere e Sapiro (2007); Spina et al. (2009)]. Para tanto, é necessária a seleção das características dissimilares entre objeto e fundo que os distinguem, de modo a realçar o máximo possível suas bordas (Figura 1.2).

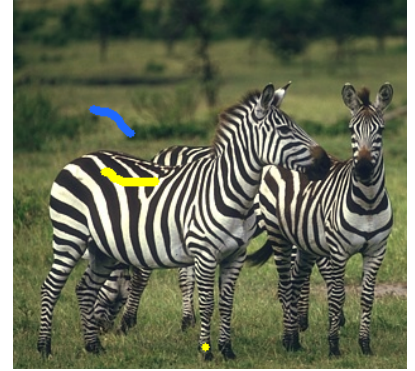

(a)

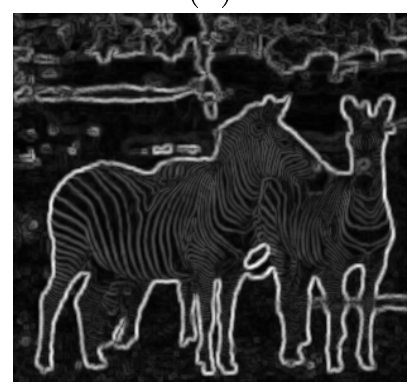

(c)

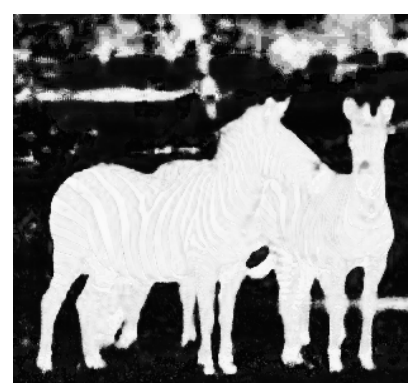

(b)

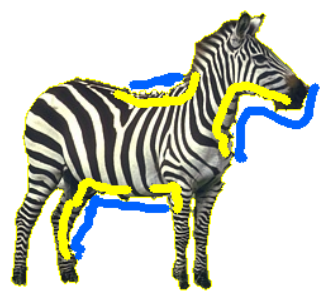

(d)

Figura 1.2: (a) Marcadores de realce selecionados pelo usuário. (b) Mapa de pertinência do objeto obtido por meio da classificação supervisionada "fuzzy". (c) Imagem dos pesos das arestas, mostrando o realce obtido na borda do objeto. Note que as transições internas entre as listras foram enfraquecidas. (d) A extração final de uma única zebra é obtida pela IFT diferencial, a partir de um novo conjunto de marcadores, explorando a conexidade ótima no espaço da imagem.

A informação de distribuição de intensidade e cor dos objetos tem sido explorada de diferentes formas: como pesos ligando os pixels a vértices terminais em um modelo de grafo estendido [Boykov e Funka-Lea (2006); Boykov e Jolly (2001)], ou na própria definição dos pesos interligando pixels vizinhos [Miranda et al. (2010); Protiere e Sapiro (2007); Spina et al. (2009)]. Existem também variações com relação a política adotada no tratamento de novas sementes selecionadas pelo usuário para corrigir erros da segmentação. Estas podem ser utilizadas ou não para recalcular os pesos das arestas do grafo. Alguns trabalhos recalculam os pesos [Boykov e Jolly (2001)]; outros consideram o realce em uma etapa separada [Miranda et al. (2010); Spina et al. (2009)], onde o usuário seleciona marcadores de treinamento (Figura 1.2); enquanto que outros usam técnicas mais sofisticadas para automatizar a seleção de sementes relevantes para fins de treinamento e realce, a partir do conjunto de marcadores fornecido pelo usuário [Spina et al. (2012)].

Todas opções apresentam seus próprios problemas: marcadores selecionados para correções locais, quando usados para recalcular pesos, podem gerar efeitos globais afetando outras regiões que já estavam corretas, por outro lado, o uso de uma etapa de treinamento pode não ser intuitivo para muitos usuários, enquanto que a última opção simplifica a interface com o usuário, porém, ao preço da maior complexidade do método (além de estar sujeita a falhas na seleção automática).

Porém o maior problema dessas abordagens é que elas se baseiam geralmente em um mo- 
delo único global da distribuição de brilho, cor, ou textura de objeto e fundo, tal como histogramas [Boykov e Jolly (2001)], mistura de gaussianas [Rother et al. (2004)], ou partição do espaço de características por meio de um classificador "fuzzy" [Miranda et al. (2010); Spina et al. (2009)]. No contexto de imagens naturais, inomogeneidades são comuns, tal como resultado das variações de luminosidade e sombras existentes. Logo, dificilmente objeto e fundo apresentam padrões de cor e brilho característicos globalmente separáveis, em função das sombras, entre outros fatores (por exemplo, camuflagem de animais em cenas naturais, e heterogeneidade do fundo) (Figura 1.3). Consequentemente, essas abordagens podem muitas vezes requerer muita interação do usuário [Peng et al. (2011)].
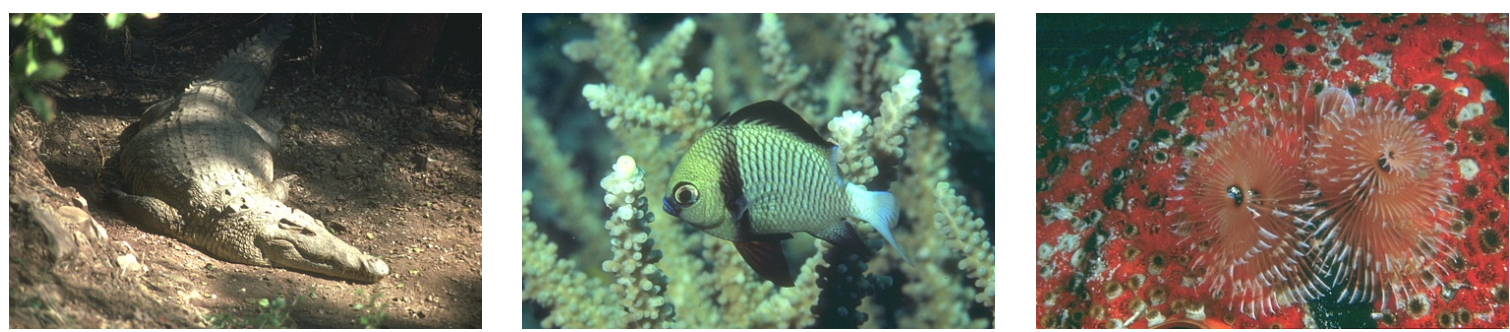

Figura 1.3: Imagens em que o objeto e o fundo têm cores muito semelhantes.

Problemas de inomogeneidade de campo também são frequentes em imagens de ressonância magnética (RM) do cérebro humano (Figura 1.4). A inomogeneidade aumenta a sobreposição entre os padrões de intensidade de objeto e fundo, no espaço de características, dificultando a segmentação de tecidos e demais estruturas cerebrais. Nesse contexto, técnicas específicas de correção de inomogeneidade podem ajudar a amenizar o problema [Axel et al. (1987); Brinkmann et al. (1998); Sled et al. (1998)]; porém estudos com imagens de 3 Tesla mostram que máscaras iniciais e cuidadosas de cérebro são necessárias para melhorar os resultados da correção de inomogeneidade [Boyes et al. (2008)]. No entanto, experimentos com imagens de 3 Tesla demonstram que as máscaras obtidas por técnicas de skull stripping são por sua vez também afetadas pela inomogeneidade de campo (Figura 1.5) [Cappabianco et al. (2012)], sendo difícil quebrar esse ciclo de dependências. Ademais, essas correções nunca são perfeitas ${ }^{1}$ e muitas vezes a avaliação é conduzida usando apenas benchmarking com imagens sintéticas [Banerjee e Maji (2013)].

No contexto de segmentação interativa, uma possível solução para o problema da heterogeneidade, a fim de superar as limitações impostas pelo uso de um único modelo global de cores, seria adotar múltiplos modelos locais de cor/intensidade, sendo cada um especializado em uma determinada região da imagem. Porém, teríamos o problema de como particionar a imagem em regiões, além das preocupações relativas a consistência da segmentação nas transições entre regiões, de modo a evitar variações abruptas nos resultados. Outro problema seria o aumento da complexidade do sistema.

Um recente método [Peng et al. (2011)] adota uma solução alternativa semelhante. Nesse trabalho os modelos de cor do objeto e fundo são atualizados iterativamente com base em regiões vizinhas, evitando a interferência de regiões distantes. Porém, muito do formalismo de otimalidade

\footnotetext{
${ }^{1}$ Experimentos mostram que métodos como, homogeneous unsharp masking (HUM) e nonparametric nonuniform intensity normalization (N3), causam alterações indevidas em imagens sem problemas de inomogeneidade, enquanto que o comportamento esperado nesse caso para um método ideal de correção seria conservar a imagem inalterada [Banerjee e Maji (2013)].
} 


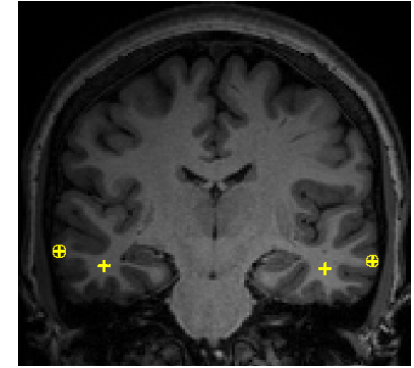

(a)

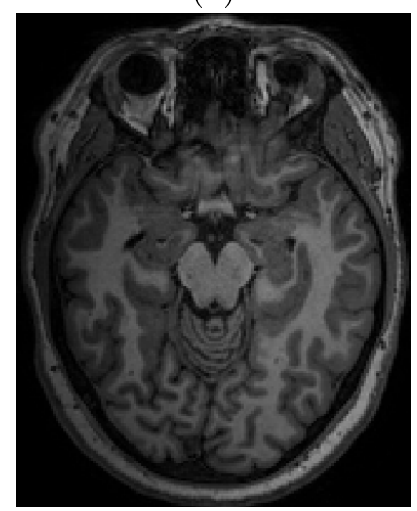

(d)

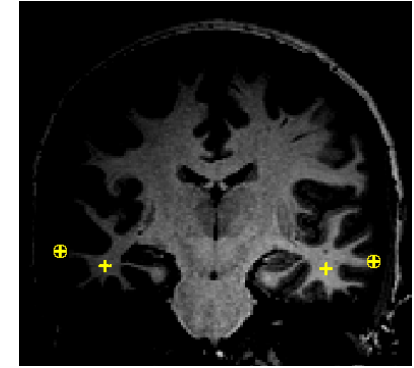

(b)

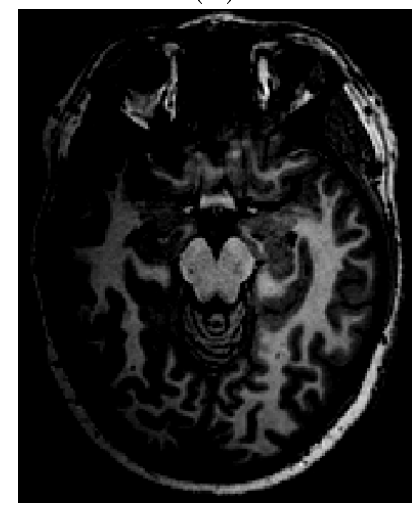

(e)

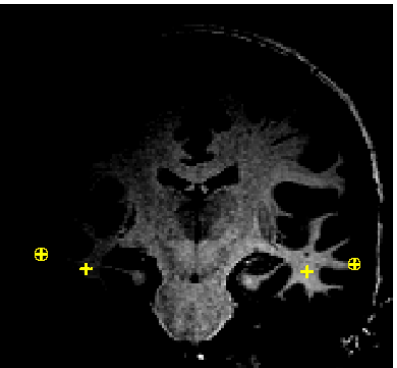

(c)

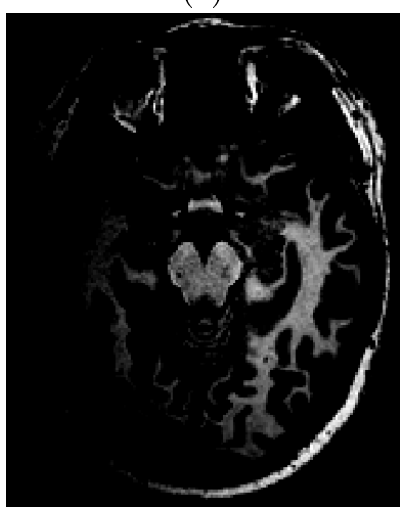

(f)

Figura 1.4: Exemplo de inomogeneidade de campo. (a) As marcas amarelas $+/ \oplus$ indicam regiões correspondentes em diferentes tecidos dos hemisférios. $\dot{A}$ esquerda, as substâncias cinzenta e branca apresentam valores de intensidade de 57 e 145, respectivamente, para os pontos marcados. Já na região direita da imagem, temos valores consideravelmente maiores, 109 e 221. (a-c) Variando as configurações de brilho e contraste é possivel melhor observar o problema. (d-f) Variações de brilho e contraste em uma fatia axial, revelando a inomogeneidade.
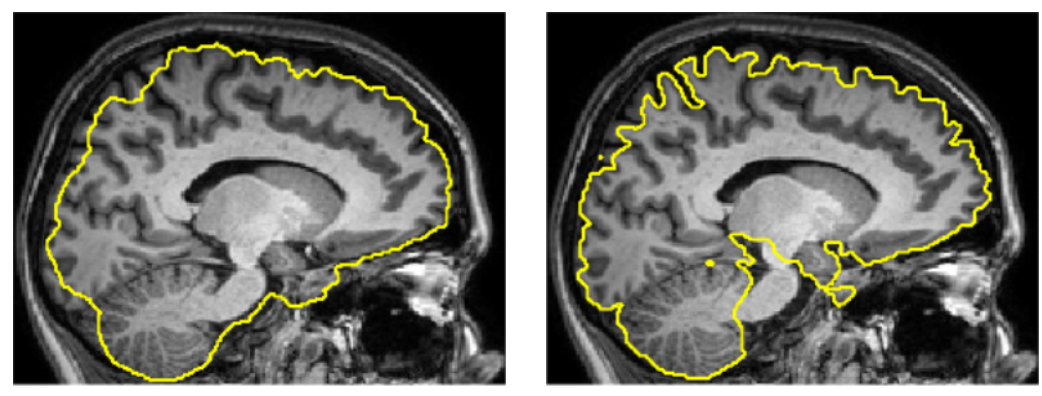

Figura 1.5: Problemas resultantes da inomogeneidade em imagens de ressonância magnética de 3 Tesla que geram erros em técnicas automáticas padrões (Brain Extraction Tool (BET) [Smith (2002)] $e$ Clouds [Miranda et al. (2008b, 2009)] respectivamente).

inicial é enfraquecido nesse método, devido à combinação heurística e complexa de muitos fatores na solução adotada.

Nesse trabalho, visamos o desenvolvimento de métodos mais naturalmente adaptativos, e que evitam muitas das complicações mencionadas anteriormente. Mais especificamente, visamos o estudo de funções de conexidade não suaves no arcabouço da transformada imagem-floresta (IFT, image foresting transform) [Falcão e Bergo (2004); Falcão et al. (2004)]. 


\subsection{Polaridade de borda}

Um problema em segmentação de imagens é a presença de bordas similares pertencentes a objetos muito próximos em uma imagem, que podem gerar resultados de segmentação não desejados. Alguns métodos baseados em borda fazem uso da informação da orientação da borda do objeto no arcabouço da IFT [Falcão et al. (2000)] para lidar com esse tipo de problema, favorecendo a segmentação em uma única direção. Nesse trabalho, propomos métodos de segmentação por região que fazem uso da informação da polaridade de borda do objeto por meio de funções de conexidade não suaves. Para isso, foi preciso estender a análise teórica da IFT por competição de sementes (IFTSC) [Miranda e Falcão (2009)] para dígrafos derivados da imagem, demonstrando a otimalidade dos cortes orientados obtidos.

\subsection{Restrições de forma}

Métodos baseados na transformada imagem-floresta [Miranda e Falcão (2009)] e similares [Protiere e Sapiro (2007)] estão entre os métodos em grafos computacionalmente mais eficientes, porém, uma vez que esses métodos carecem de restrições de regularização de borda, seus resultados muitas vezes apresentam aspectos irregulares indesejados. Outros métodos procuram contornar essa limitação incorporando em suas formulações alguma componente de regularização, como por exemplo, a minimização de uma energia de tensão na fronteira do objeto [Boykov e Jolly (2001); Grady (2006)], porém ao preço de elevar a complexidade computacional, ou do preço de introduzir um viés de encolhimento dos objetos.

Uma outra forma de tratar o problema é através da incorporação de restrições de forma, como a convexidade geodésica em estrela [Gulshan et al. (2010)]. Nesse trabalho, mostramos como é possível incorporar essas restrições na IFT, sem comprometer a complexidade do algoritmo, favorecendo a segmentação de objetos com forma mais regular.

\subsection{Organização do Trabalho}

No Capítulo 2 apresentamos conceitos básicos e notações referentes a imagens digitais e teoria de grafos, os quais serão usados nos capítulos seguintes. A metodologia adotada usa a IFT, a qual é apresentada no Capítulo 3. Nos demais capítulos são apresentadas as contribuições do presente projeto. No Capítulo 4, apresentamos as funções de conexidade não suaves e propomos um diagrama de classificação das mesmas [Mansilla et al. (2013a)]. No Capítulo 5 provamos algumas propriedades teóricas para algumas regiões do diagrama, que contém algumas funções não suaves que demonstraram bons resultados no tratamento dos problemas de inomogeneidade para segmentação interativa [Mansilla et al. (2013a)]. No Capítulo 6 apresentamos algumas funções não suaves que permitem a incorporação da polaridade de borda na segmentação pela IFT, resultando na técnica de Segmentação pela transformada imagem-floresta orientada(OIFT) [Mansilla e Miranda (2013a); Miranda e Mansilla (2014)]. No Capítulo 7 apresentamos uma variação do algoritmo da IFT que permite a incorporação de restrições de forma de modo a favorecer a segmentação de objetos com forma mais regular, resultando na técnica de Segmentação pela transformada imagem-floresta com restrições de convexidade geodésica em estrela (GSC-IFT) [Mansilla et al. (2013b)]. No Capítulo 8 mostramos que a restrição de convexidade geodésica em estrela, apresentada no capítulo anterior, 
pode também ser obtida mediante o emprego de funções de conexidade não suaves no arcabouço da transformada imagem-floresta, além de permitir o tratamento simultâneo da polaridade de borda (Capítulo 6), resultando na técnica da OIFT com restrições de convexidade geodésica em estrela (GSC-OIFT) [Mansilla e Miranda (2013b)]. Finalmente, no Capítulo 9 discutimos algumas conclusões obtidas neste trabalho. 


\section{Capítulo 2}

\section{Conceitos}

Nesta seção são apresentados os conceitos básicos e notações que serão usados ao longo do documento. No entanto, leitores mais familiarizados podem ir diretamente para o Capítulo 3.

\section{$2.1 \quad$ Imagem digital}

Uma imagem digital $\hat{I}$ é um par $(\mathcal{I}, \vec{I})$, onde $\mathcal{I}$ é um conjunto de pontos do $Z^{n}$ (domínio da imagem), denominados spels (space elements), e $\vec{I}$ é um mapeamento vetorial que associa a cada spel $t \in \mathcal{I}$ um conjunto de $m$ valores escalares $I_{i}(t), i=1,2, \ldots, m$, associados com alguma propriedade física. O valor de $n$ refere-se à dimensão da imagem e o valor de $m$ ao número de bandas.

Por exemplo, uma foto colorida gera uma imagem com $n=2$ e $m=3$, onde $I_{i}(t) \operatorname{com} i=1,2,3$ representa os valores de luz refletida em $t \in \mathcal{I}$ nos comprimentos de onda do vermelho, verde, e azul, respectivamente.

\subsubsection{Imagem em tons de cinza}

Uma imagem $\hat{I}=(\mathcal{I}, I)$ em tons de cinza (por exemplo, foto preto e branco, imagem de ultrasom, fatia tomográfica) e bidimensional $\left(\mathcal{I} \subset Z^{2}\right)$ possui apenas uma banda $I(m=1)$, onde os spels são chamados pixels (picture elements). Essas imagens podem ser representadas na forma de uma matriz de tamanho $N \times M$ pixels ( $N$ linhas e $M$ colunas).

Os valores $I(t)$ de cada pixel $t$ são obtidos por amostragem e quantização de uma função contínua $I_{c}(x, y)$ que descreve a propriedade física correspondente em uma dada região do espaço. No caso de uma foto temos o brilho, e no caso de uma tomografia de Raios-X temos a densidade do tecido. Valores altos são apresentados na tela como pixels claros e valores baixos como pixels escuros.

\section{Amostragem e resolução espacial}

Cada pixel é amostrado a intervalos $\left(\Delta_{x}, \Delta_{y}\right.$ ) (por exemplo, $\Delta_{x}=\Delta_{y}=1 \mathrm{~mm}$ ). Quanto menor for o intervalo de amostragem para uma mesma região do espaço, maior será a resolução espacial da imagem. Observe que neste caso, o tamanho $N$ x $M$ da imagem também é maior, mas se uma imagem tem mais pixels que outra, não implica que tenha maior resolução. 


\section{Quantização e resolução radiométrica}

Os valores de $I_{c}(x, y)$ amostrados são quantizados em $2^{b}$ níveis de cinza, onde $b$ é chamado profundidade da imagem em bits (por exemplo, $b=8$, profundidade de 8 bits, significa $2^{8}=256$ níveis de cinza). Quanto menor o intervalo da propriedade física $\Delta_{I_{c}}$ correspondente a variação de uma unidade da escala de cinza, maior é a resolução radiométrica da imagem.

\subsubsection{Imagem multidimensional}

Uma imagem $\hat{I}=(\mathcal{I}, I)$ em tons de cinza e multidimensional define domínio de amostragem $\mathcal{I} \subset Z^{n}$, para $n>2$. Por exemplo, uma sequência espacial de fatias tomográficas é uma imagem tridimensional $(n=3)$, e uma sequência espacial e temporal de fatias tomográficas é uma imagem tetradimensional $(n=4)$. No primeiro caso, os spels são chamados de voxels (volume element). $\mathrm{O}$ intervalo de amostragem ao longo do eixo temporal define a resolução temporal da imagem. Quanto menor o intervalo, maior é a resolução.

\subsubsection{Imagem multibanda}

Uma imagem $\hat{I}=(\mathcal{I}, \vec{I})$ é multibanda quando necessariamente $\vec{I}$ associa a cada spel $t$ mais de um valor escalar, isto é, $I_{i}(t)$ onde $i=1,2, \ldots, m$ com $m>1$. Por exemplo, imagens de satélite com o sensor Thematic Mapper, têm $n=2$ e $m=7$, as quais possuem bandas que correspondem a imagens cinza obtidas nos comprimentos de onda do azul, vermelho, verde, infravermelho, infravermelho próximo, termal, e ultravioleta. O intervalo de amostragem define a resolução espectral. No caso de um vídeo colorido temos uma imagem multidimensional e multibanda, pois mistura espaço e tempo para formar 3 dimensões $(n=3)$ e cada quadro possui 3 bandas de cor $(\mathrm{m}=3)$.

\subsubsection{Imagem de rótulos}

A segmentação de uma imagem $\hat{I}=(\mathcal{I}, \vec{I})$ baseada em região pode ser vista como um mapeamento que associa para todo pixel $t \in \mathcal{I}$ um inteiro $L(t)$, denominado rótulo, cujo valor é um identificador único de cada objeto (incluindo o fundo). Neste caso, a segmentação é dita hard porque cada pixel $t$ só pertence a um único objeto. Algumas abordagens estendem este conceito para fuzzy, onde cada pixel $t$ pertence a todos os objetos com diferentes graus de pertinência. A segmentação hard pode ser representada como uma imagem em tons de cinza $\widehat{L}=(\mathcal{I}, L)$ chamada imagem de rótulos.

\subsection{Noções de grafos}

\subsubsection{Grafo}

Um grafo $G$ é um par $(\mathcal{V}, \xi)$, onde $\mathcal{V}$ é um conjunto finito não vazio de vértices (ou nós) e $\xi$ é um conjunto composto por pares de elementos distintos de $\mathcal{V}$, denominados arestas. Usamos $|\xi|$ para denotar o número de arestas do grafo, e $|\mathcal{V}|$ é o número de vértices. 


\subsubsection{Grafo direcionado}

Um grafo $G$ é direcionado (dígrafo) ou orientado, quando o conjunto de arestas $\xi$ está composto de pares ordenados de elementos de $\mathcal{V}$ (isto é, as arestas $(u, v)$ e $(v, u)$ são consideradas distintas). Caso contrário dizemos que o grafo é não direcionado. Assim, uma aresta $e=(u, v)$ é dita divergente de $u$ e convergente a $v$ em dígrafos, enquanto que, em grafos não direcionados, a aresta $e$ é dita incidente a $u$ e $v$.

\subsubsection{Grafo ponderado}

Um grafo $G$ é ponderado, se possui valores (pesos) associados às arestas. Seja $\omega(u, v)$ o peso associado a cada aresta $(u, v) \in \xi$, se $\omega(u, v)=\omega(v, u)$ nós temos um grafo ponderado não direcionado, caso contrário o grafo é ponderado direcionado.

\subsubsection{Subgrafo}

Um subgrafo $G_{2}=\left(\mathcal{V}_{2}, \xi_{2}\right)$ de um grafo $G_{1}=\left(\mathcal{V}_{1}, \xi_{1}\right)$ é um grafo tal que $\mathcal{V}_{2} \subseteq \mathcal{V}_{1}$ e $\xi_{2} \subseteq \xi_{1}$. Se $\mathcal{V}_{2}=\mathcal{V}_{1}$ então $G_{2}$ é chamado de subgrafo gerador ou subgrafo de espalhamento.

\subsubsection{Relação de adjacência}

Uma relação de adjacência $A$ é uma relação binária entre vértices no grafo $G$. Assim, um vértice $v$ é adjacente a (ou vizinho de) um vértice $u$ se existe uma aresta $e \in \xi$ denotada por $e=(u, v)$. No entanto, a relação de adjacência entre vértices pode ser não simétrica ( $v$ pode ser ajacente a $u$ sem que $u$ seja ajacente a $v$, ou seja $(v, u) \notin \xi)$. No caso de grafos não direcionados, a relação de adjacência é simétrica.

Cada vértice $u \in V$ tem associado um conjunto de todos os vértices $v$ adjacentes (vizinhos) a ele, o qual define uma vizinhança que pode ser representada como uma lista de adjacências $A(u)=\{v \in \mathcal{V} \mid(u, v) \in \xi\}$, assim $v \in A(u)$ indica que o vértice $v$ é adjacente a $u$ em $G$. O número de vértices adjacentes a $u$ define o grau dele, que será denotado por grau(u), no caso de dígrafos é denominado grau de saída.

\subsubsection{Caminho}

Uma sequência de vértices $\left\langle v_{0}, v_{1}, v_{2}, \ldots, v_{k}\right\rangle$ tal que $\left(v_{i-1}, v_{i}\right) \in \xi$ para $i=1,2, \ldots, k$, é denominado caminho de $v_{0}$ a $v_{k}$ em um grafo $G=(\mathcal{V}, \xi)$. Diz-se então que $v_{0}$ alcança ou atinge $v_{k}$. O valor de $k$ é o comprimento do caminho, o qual está formado por $k+1$ vértices e $k$ arestas $\left(v_{0}, v_{1}\right),\left(v_{1}, v_{2}\right), \ldots,\left(v_{k-1}, v_{k}\right)$. Se todos os vértices do caminho são distintos, nós temos um caminho simples ou elementar.

\subsubsection{Ciclo}

Um caminho $\left\langle v_{0}, v_{1}, v_{2}, \ldots, v_{k}\right\rangle$ forma um ciclo, se $v_{0}=v_{k}$ e $k \geq 3$ no caso de grafos não direcionados. Se nenhum vértice do caminho é repetido, com exceção do primeiro e do último que coincidem, também diz-se que o ciclo é simples ou elementar. Se o grafo não possui ciclos simples, então o grafo é denominado acíclico. 


\subsubsection{Grafo conexo}

Um grafo $G$ não direcionado é conexo, se existe um caminho simples entre cada par de vértices de $G$. Caso contrário o grafo $G$ é dito desconexo. Os subgrafos maximais conexos de um grafo $G$ não direcionado são chamados de componentes conexos.

\subsection{9 Árvore}

Uma árvore é um grafo $T=(\mathcal{V}, \xi)$ não direcionado, conexo, e acíclico. Se uma árvore é um subgrafo gerador de outro grafo, ela é denominada de árvore geradora ou de espalhamento. Uma árvore é chamada de enraizada quando algum vértice $v \in \mathcal{V}$ é escolhido como especial, o qual é denominado raiz da árvore.

\subsubsection{Floresta}

Uma floresta é um conjunto de árvores. Também podemos dizer que todo grafo não direcionado acíclico, sendo ou não conexo, é uma floresta.

\subsubsection{Corte}

Um corte $\left(\mathcal{V}^{\prime}, \mathcal{V}-\mathcal{V}^{\prime}\right)$ de um grafo $G=(\mathcal{V}, \xi)$ não direcionado é uma partição de $\mathcal{V}$. Diz-se que, uma aresta $(u, v) \in \xi$ cruza o corte $\left(\mathcal{V}^{\prime}, \mathcal{V}-\mathcal{V}^{\prime}\right)$, se um de seus vértices pertence a $\mathcal{V}^{\prime}$ e o outro vértice pertence a $\mathcal{V}-\mathcal{V}^{\prime}$

\subsection{Grafos a partir de imagens}

Uma imagem $\hat{I}=(\mathcal{I}, \vec{I})$ pode ser vista como um grafo $G=(\mathcal{V}, \xi)$, onde os vértices em $\mathcal{V}$ correspondem aos spels em $\mathcal{I}$ e as arestas em $\xi$ são definidas por uma relação de adjacência $A$; que, em geral, depende das posições relativas dos spels ( $A$ é invariante à translação), e opcionalmente de outras propriedades locais da imagem (por exemplo, cor e gradiente), mas neste último caso $A$ seria variante à translação.

Por exemplo, considere a distância Euclideana entre dois pixels $p$ e $q$ denotada por $d(p, q)$. Podemos considerar $\xi$ como sendo composto por todos os pares de pixels $(p, q) \in \mathcal{I} \times \mathcal{I}$ tais que $p \neq q$ e $d(p, q) \leq \rho$, onde $\rho$ é uma constante dada. Essas adjacências são chamadas de adjacências Euclideanas, e correspondem a uma relação simétrica e invariante a translação. As Figuras 2.1(a), 2.1(b) e 2.1(c) mostram alguns exemplos em $2 D$, para $\rho=1$ temos uma vizinhança 4 e para $\rho=\sqrt{2}$ temos uma vizinhança 8. Na Figura 2.1(d) podemos observar um exemplo para o caso $3 D$ com $\rho=1$, o qual corresponde a uma vizinhança 6 .

Do exemplo anterior temos que $A$ pode ser armazenada em uma única estrutura que guarda os deslocamentos relativos $d x$ e $d y$, ou seja, $q-p \in\{(d x[i], d y[i])\}$, para $i=0,1, \ldots,|A(p)|-1$. No caso $3 D$ também temos $d z$. Por exemplo, na Figura 2.1(a) um pixel $q\left(x_{1}, y_{1}\right)$ é adjacente a um outro pixel $p\left(x_{2}, y_{2}\right)$ se e somente se $q-p=\left(x_{1}-x_{2}, y_{1}-y_{2}\right) \in\{(-1,0),(0,-1),(1,0),(0,1)\}$. 


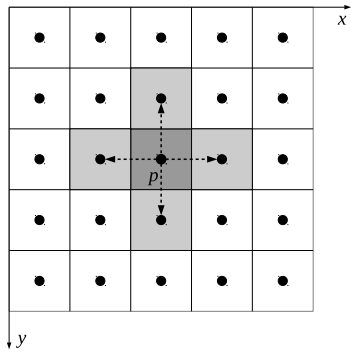

(a)

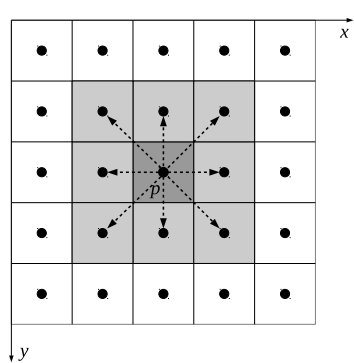

(b)

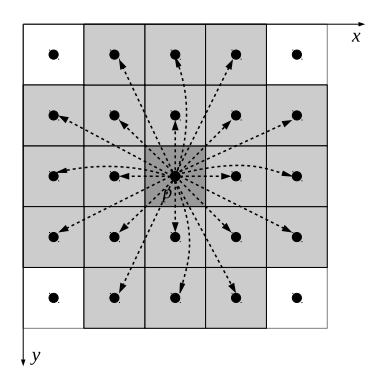

(c)

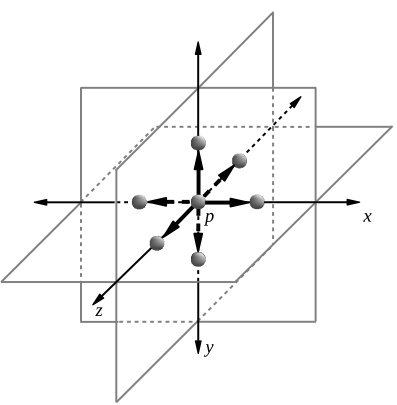

(d)

Figura 2.1: Adjacências Euclideanas: caso $2 D$ com raio $(a) \rho=1$, (b) $\rho=\sqrt{2}$, (c) $\rho=\sqrt{5}$, e caso $3 D$ com raio (d) $\rho=1$.

\subsection{Objeto}

Um objeto na imagem $\hat{I}=(\mathcal{I}, \vec{I})$ é um subconjunto de $\mathcal{I}$ formado por um ou mais componentes conexos. Uma borda de objeto é um conjunto de pixels do seu interior que possui ao menos um pixel adjacente no exterior. Assim, um objeto pode ser representado por suas bordas ou pelos pixels que compõem seu interior.

\subsection{Avaliação do desempenho entre métodos de segmentação}

\subsubsection{Acurácia}

O desempenho de um método pode ser definido de várias formas, como por exemplo: acurácia, tempo de execução, entre outros. A acurácia é o grau de proximidade entre o resultado obtido e o valor verdadeiro. Na literatura existem diferentes medidas de acurácia, pois ainda não existe uma medida universal que seja melhor para todos os casos de avaliação [Stehman (1997)]. Geralmente, as medidas de acurácia possuem valores em um intervalo de $[-1,1]$ ou $[0,1]$, onde 0 ou -1 representam a pior acurácia, sendo isso o caso de imagens incompatíveis, e 1 mostra a melhor acurácia, para o caso de imagens absolutamente similares.

Neste trabalho fazemos uso do Coeficiente de Dice [Dice (1945)] para avaliar a acurácia dos métodos de segmentação de imagens, também conhecido como F-score ou Fmeasure [Labatut e Cherifi (2012); Rauber et al. (2013); van Rijsbergen (1979)], Coeficiente de similaridade de Sørensen [Sørensen (1948)] e Índice de acurácia média de Hellden [Hellden (1980)].

O Coeficiente de Dice esta representado pela seguinte relação:

$$
D=\frac{2|X \bigcap Y|}{|X|+|Y|}
$$

$\mathrm{Ou}$

$$
D=\frac{2|X \bigcap Y|}{|X \bigcap Y|+|X \bigcup Y|}
$$

onde $X$ representa o conjunto de pixels rotulados como objeto na segmentação pelo método analisado e $Y$ representa os pixels rotulados como objeto na imagem esperada, isto é, a segmentação ideal para uma imagem, conhecida como gabarito da imagem, a qual é uma imagem binária (branco 
no objeto e preto no fundo) geralmente obtida mediante uma segmentação manual.

Por exemplo, consideremos uma imagem (Figura 2.2(a)) com gabarito conhecido (Figura 2.2(b)), cujo resultado da segmentação por um método específico $M_{1}$ é a imagem da Figura 2.2(c). Calculando o valor do coeficiente de Dice (Equação 2.1) para o resultado da segmentação pelo método $M_{1}$ e o gabarito da imagem avaliada temos:

$$
\begin{aligned}
D & =\frac{2(34)}{42+40} \\
D & =0.83
\end{aligned}
$$

o qual representa o valor da medida da acurácia para o método $M_{1}$.

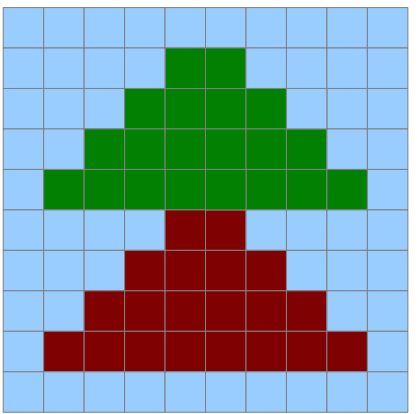

(a)

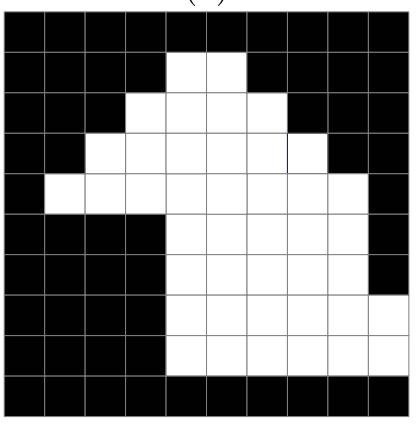

(c)

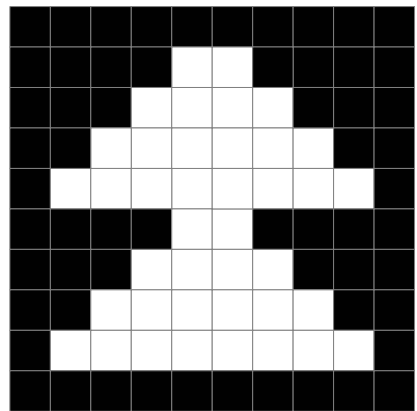

(b)

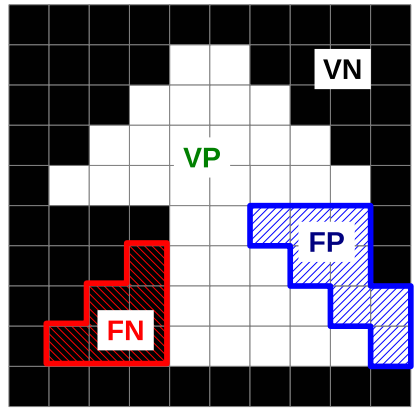

(d)

Figura 2.2: Exemplo de segmentação pelo método $M_{1}$ para uma imagem de avaliação (a), com gabarito conhecido (b), cujo resultado da segmentação (c) apresenta diferentes conjuntos que representam pixels do objeto corretamente rotulados (VP - verdadeiros positivos), pixels do fundo corretamente rotulados (VN verdadeiros negativos), pixels do fundo incorretamente rotulados (FP-falsos positivos), e pixels do objeto incorretamente rotulados ( $F N$-falsos negativos).

Também, na Figura 2.2(d), podemos observar que o resultado da segmentação apresenta conjuntos de pixels rotulados incorretamente (erro da segmentação), os quais são conhecidos como falsos positivos (FP) e falsos negativos (FN), de modo que os pixels corretamente rotulados como objeto e fundo são chamados de verdadeiros positivos (VP) e verdadeiros negativos (VN) respectivamente. Esses conjuntos podem ser visualizados em uma matriz de confusão (Figura 2.3), onde se o pixel no gabarito da imagem é positivo (pertence ao objeto) e é rotulado como positivo na imagem obtida pela segmentação por um método específico, então temos um verdadeiro positivo; se o mesmo é rotulado como negativo (pertence ao fundo), temos um falso negativo. Se o pixel no gabarito da imagem é negativo e é rotulado como negativo, então temos um verdadeiro negativo; e caso seja rotulado como positivo temos um falso positivo.

Considerando a matriz de confusão, o coeficiente de Dice também pode ser expressado pela 


\begin{tabular}{|c|c|c|c|}
\hline & & \multicolumn{2}{|c|}{ Imagem obtida da segmentação } \\
\hline & & $\begin{array}{l}\text { Objeto } \\
\text { (positivo) }\end{array}$ & $\begin{array}{l}\text { Fundo } \\
\text { (negativo) }\end{array}$ \\
\hline \multirow{2}{*}{ 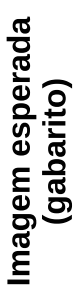 } & $\begin{array}{c}\text { Objeto } \\
\text { (positivo) }\end{array}$ & $\begin{array}{c}\text { VP } \\
\text { verdadeiro positivo } \\
\text { corretamente segmentado como objeto }\end{array}$ & $\begin{array}{c}\text { FN } \\
\text { falso negativo } \\
\text { incorretamente não segmentado como objeto }\end{array}$ \\
\hline & $\begin{array}{l}\text { Fundo } \\
\text { (negativo) }\end{array}$ & $\begin{array}{c}\text { FP } \\
\text { falso positivo } \\
\text { incorretamente segmentado como objeto }\end{array}$ & $\begin{array}{c}\text { VN } \\
\text { verdadeiro negativo } \\
\text { corretamente não segmentado como objeto }\end{array}$ \\
\hline
\end{tabular}

Figura 2.3: Matriz de confusão.

Equação 2.4 (F-measure).

$$
D=\frac{2|V P|}{2|V P|+|F N|+|F P|}
$$

Para avaliar a acurácia de diferentes métodos de segmentação, calculamos o coeficiente de Dice para cada um deles e comparamos seus resultados para ver qual deles apresenta a maior acurácia.

Na segmentação interativa de imagens precisamos de marcadores para realizar as segmentações. A fim de obter uma avaliação o mais imparcial possível, e passível de reprodutibilidade, consideramos procedimentos automáticos de seleção de marcadores. As duas abordagens comumente adotadas na literatura são marcadores obtidos pela erosão do objeto e fundo no gabarito da imagem [Sinop e Grady (2007)], e marcadores obtidos via um usuário robô [Gulshan et al. (2010)].

\subsubsection{Marcadores escolhidos por erosão do gabarito}

Como temos visto até agora, para obter o valor da acurácia de um método específico para uma dada imagem, precisamos do gabarito dessa imagem e da imagem binária resultante da segmentação. Dado que nosso interesse é a segmentação interativa, precisamos de marcadores. Uma forma de obter os marcadores é realizando a erosão do objeto presente no gabarito da imagem e a erosão do complemento da imagem do gabarito (fundo) com o objetivo de obter os marcadores do objeto e fundo respectivamente [Sinop e Grady (2007)]. Isto é, os marcadores do objeto serão representados pela borda do objeto erodido no gabarito da imagem e os marcadores do fundo serão representados pela borda do fundo erodido (Figura 2.4). A magnitude do raio de erosão não precisa ser a mesma para o objeto e fundo, sendo possível usar raios de erosão assimétricos, a fim de não gerar sementes equidistantes da borda, pois este tipo de cenário pode favorecer alguns métodos, viesando a análise.

Para comparar o desempenho de vários métodos, podemos fazer uso de uma curva de acurácia, obtida variando o parâmetro de raio. Por exemplo, o gráfico ilustrativo da Figura 2.5 mostra as curvas de acurácia, para raios de erosão simétricos, de dois métodos de segmentação hipotéticos $\left(M_{1}\right.$ e $M_{2}$ ). Do mesmo gráfico podemos observar que o método $M_{2}$ apresenta melhor acurácia do que o método $M_{1}$, uma vez que sua curva se encontra mais acima no gráfico. Outro ponto a ser observado é que, em geral, a medida que o raio de erosão aumenta a acurácia diminui, em função do aumento da região a ser rotulada. 


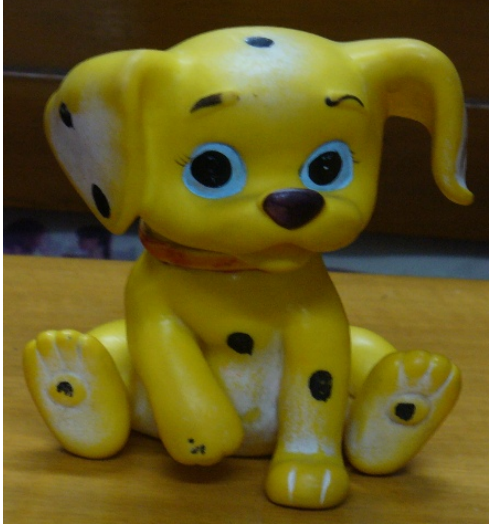

(a)

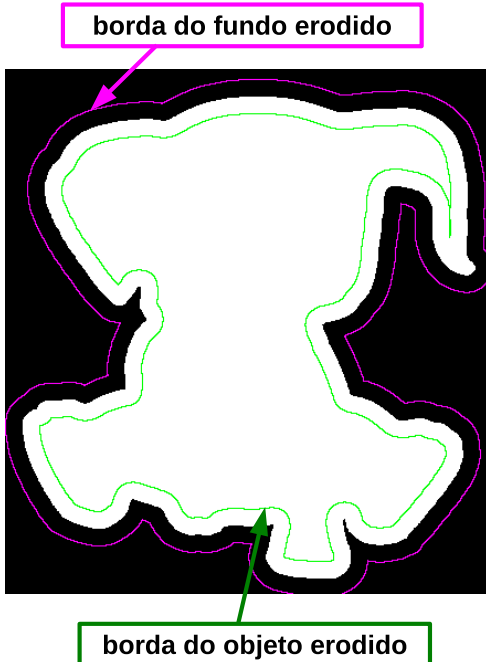

(c)

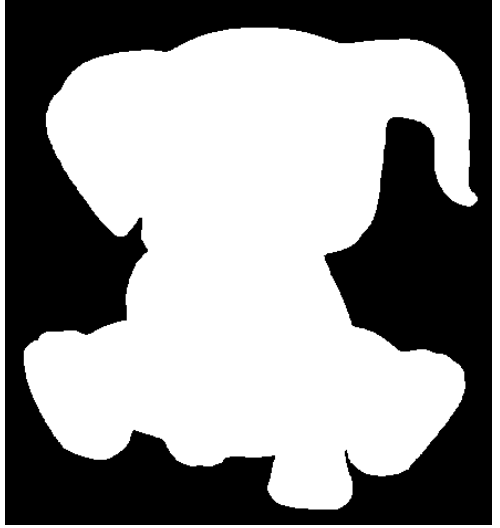

(b)

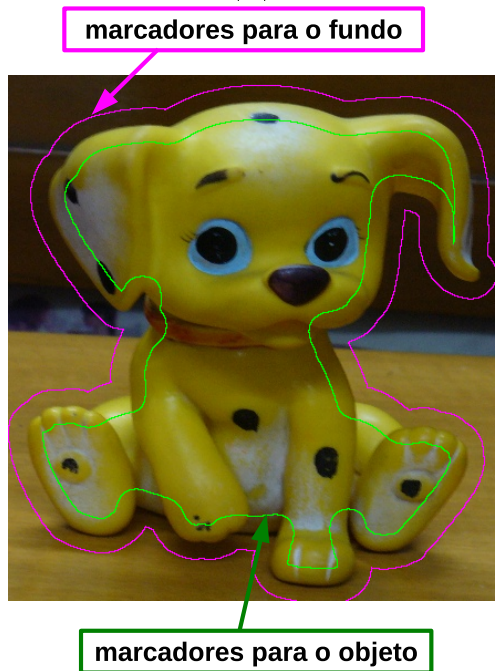

(d)

Figura 2.4: Exemplo de marcadores escolhidos por erosão do gabarito: (a) Imagem a segmentar. (b) Gabarito da imagem. (c) Bordas do objeto e fundo erodidos com raio = 15 pixels. (d) Marcadores resultantes.

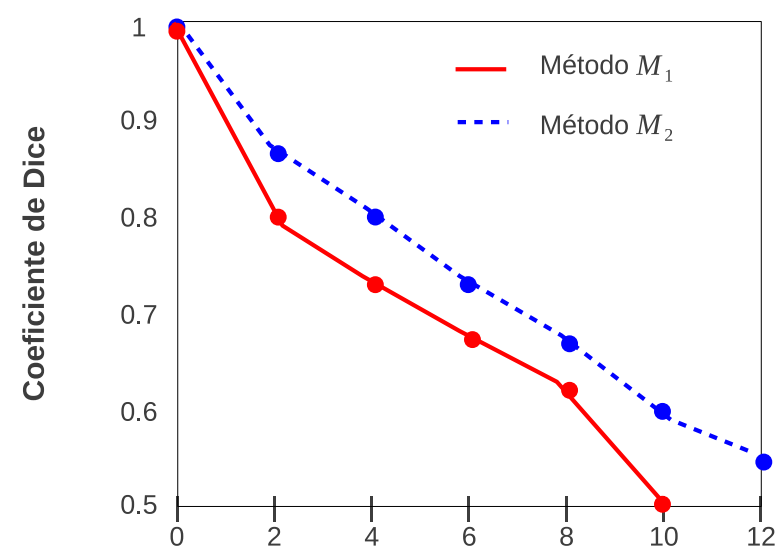

Raio de erosão (pixels)

Figura 2.5: Exemplo de curvas da acurácia para os métodos hipotéticos $M_{1}$ e $M_{2}$ fazendo uso de marcadores obtidos pela erosão do gabarito com diferentes valores de raio de erosão.

A vantagem desse tipo de abordagem é que ela garante que todos os métodos são avaliados com base nas mesmas sementes. 


\subsubsection{Marcadores escolhidos por um usuário robô}

Uma outra forma de obter os marcadores para segmentar as imagens com um método determinado é fazendo uso de um usuário robô [Gulshan et al. (2010)], o qual simula o comportamento de um usuário humano para a segmentação interativa de imagens. Para o caso de uma imagem 2D, inicialmente o usuário robô coloca marcadores circulares no objeto e fundo em pontos mais distantes da borda do objeto e do fundo respectivamente, com base no gabarito da imagem. Feito isso, a segmentação é calculada e as regiões incorretamente segmentadas são identificadas (falsos positivos e falsos negativos). A maior componente conexa de erro é selecionada para colocar um marcador do objeto adicional caso a componente seja um falso negativo ou um marcador do fundo caso se trate de um falso positivo, de modo que o novo marcador é colocado no ponto mais distante da borda do componente para atualizar a segmentação. O procedimento se repete nas iterações sucessivas gerando uma curva de acurácia.

Apesar de essa abordagem simular melhor um ambiente interativo, não temos a garantia que as sementes usadas são as mesmas, quando comparamos diferentes métodos de segmentação.

Um exemplo do comportamento do usuário robô é mostrado na Figura 2.6. 


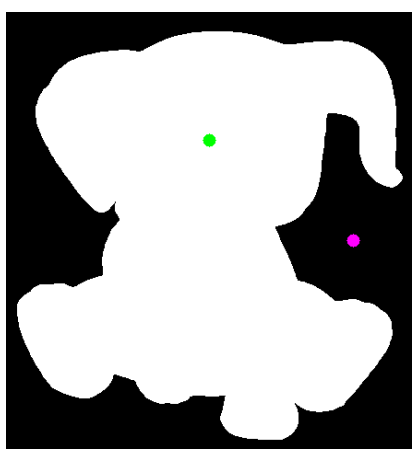

(a)

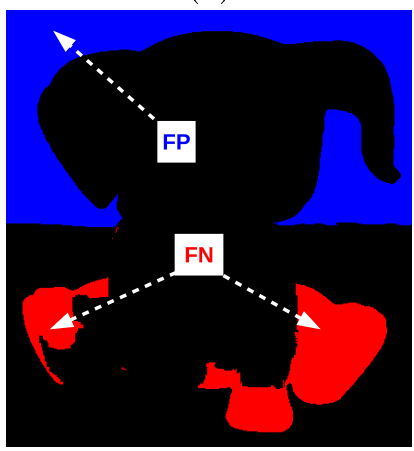

(d)

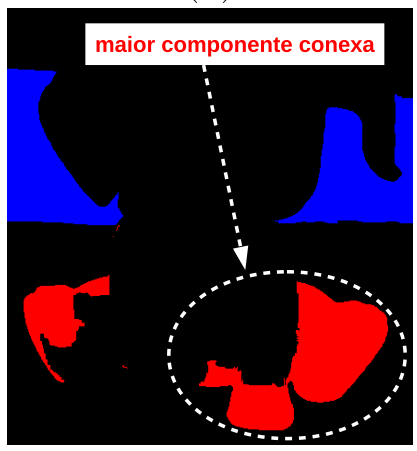

(g)

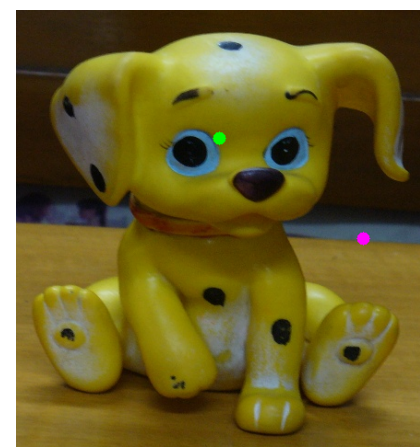

(b)

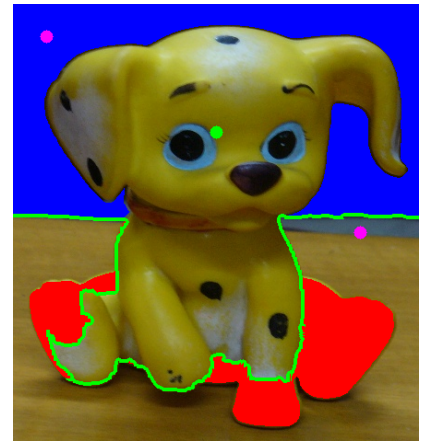

(e)

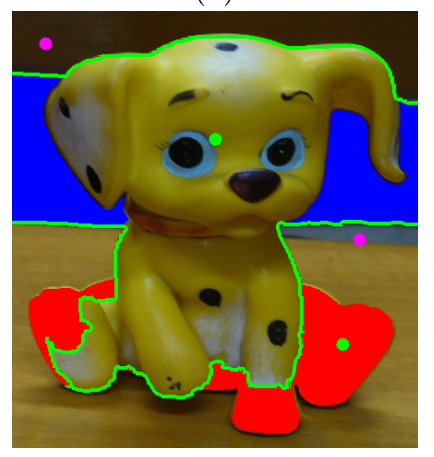

(h)

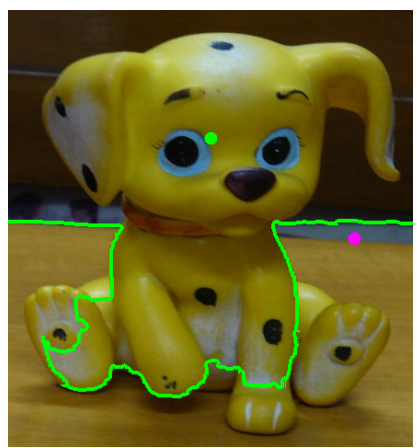

(c)

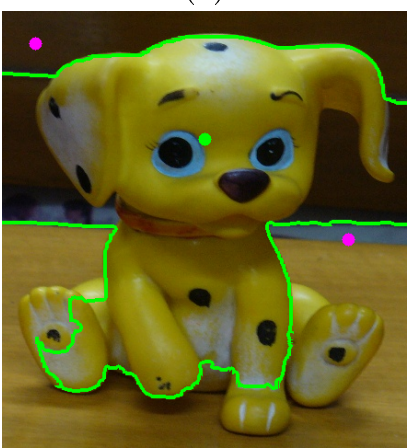

(f)

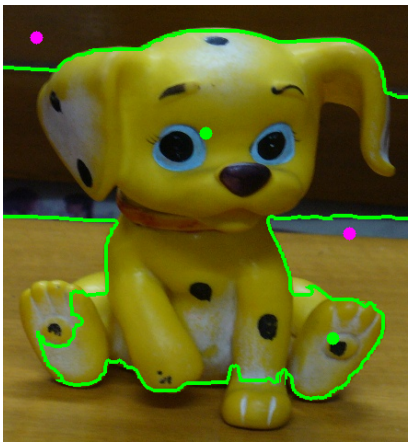

(i)

Figura 2.6: Exemplo de marcadores escolhidos por um usuário robô para a mesma imagem da Figura 2.4(a): Em (a) o usuário robô identifica os pontos mais distantes da borda do objeto e fundo considerando o gabarito da imagem. Em (b) são mostrados os marcadores colocados pelo usuário robô na imagem. Em (c) a borda verde representa o resultado da segmentação na primeira iteração. Em (d) e (g) o robô identifica as regiões incorretamente segmentadas, isto é, as regiões em azul representando os falsos positivos (FP) e as regiões em vermelho representando os falsos negativos (FN). Em (e) o robô coloca um novo marcador na maior componente conexa. Isto é, um marcador do fundo na região azul. Em (f) é mostrado o resultado da segmentação com o marcador adicional (segunda iteração). Em (h) o robô coloca um marcador do objeto na região em vermelho (maior componente conexa). Em (i) temos o resultado da segmentação na terceira iteração. 


\section{Capítulo 3}

\section{Transformada imagem-floresta}

A metodologia proposta está baseada na transformada imagem-floresta (IFT, Image Foresting Transform), a qual é uma ferramenta geral para modelar, implementar e avaliar operadores de processamento de imagens $2 \mathrm{D} / 3 \mathrm{D}$ baseados em conexidade.

A IFT unifica e estende várias técnicas de análise de imagens que, muito embora baseadas em conceitos similares, são normalmente apresentadas como métodos não relacionados. Ela reduz o problema de processamento de imagem (por exemplo, filtragem [Falcão et al. (2001)], segmentação [Falcão e Bergo (2004); Miranda et al. (2011); Spina et al. (2012)], representação de formas [Falcão e da Cunha (2001)]) ao cálculo de uma floresta de caminhos ótimos (custo mínimo ou força de conexidade máxima) no grafo derivado da imagem, seguido de um processamento adequado de seus atributos.

Neste capítulo, nós apresentaremos a IFT de modo mais específico para o problema de segmentação baseada em regiões.

\subsection{Definição}

Na IFT uma imagem 2D/3D pode ser vista como um grafo $G=(\mathcal{I}, \xi)$ onde os nós/vértices são os pixels/voxels da imagem no seu domínio $\mathcal{I} \subset Z^{n}$ e as arestas são os pares de pixels $(s, t) \in \xi$ definidos por uma relação de adjacência $A$ (por exemplo, vizinhança 4 em 2D, ou 6 em 3D). Cada aresta $(s, t) \in \xi$ pode ter um peso $\omega(s, t) \geq 0$ associado, que pode ser dado por uma medida de dissimilaridade entre os pixels $s$ e $t$ (por exemplo, $\omega(s, t)=|I(t)-I(s)|$ para uma imagem com um só canal, com valores dados por $I(t))$. Um caminho simples $\pi_{t}$ nesse grafo é uma sequência de pixels distintos e adjacentes com término no pixel $t$. Todos os caminhos considerados nesse trabalho são caminhos simples, isto é, caminhos que passam por cada vértice não mais que uma vez. Assim, usamos a notação $\pi_{t}=\langle t\rangle$ para indicar um caminho composto por um único vértice, denominado caminho trivial, e $\pi_{t}=\pi_{s} \cdot\langle s, t\rangle$ para indicar a extensão de um caminho $\pi_{s}$, com término em $s$, por uma aresta $(s, t) \in \xi$ (Figura 3.1(a)). Cada caminho $\pi_{t}$ possui um valor de conexidade (ou custo) $f\left(\pi_{t}\right)$ associado, definido por uma função de custo de caminho $f$, que normalmente depende de propriedades locais da imagem, tais como cor, gradiente, e posição do nó ao longo do caminho.

No framework da IFT consideramos um mapa de predecessores que é uma função $P$ que associa a cada pixel $t \in \mathcal{I}$ algum outro pixel adjacente em $\mathcal{I}$, ou um marcador distinto nil $\notin \mathcal{I}$. Nesse último caso $t$ é dito ser uma raiz do mapa (Figura 3.1(b)). De modo que uma floresta de espalhamento é um mapa $P$ de predecessores o qual não contém ciclos, ou seja, aquele que leva todo pixel para nil em 
um número finito de iterações (Figura 3.1(c)). Assim, para qualquer pixel $t \in \mathcal{I}$, a floresta $P$ define um caminho induzido $\pi_{t}^{P}$ recursivamente como $\langle t\rangle$, se $P(t)=n i l$, e $\pi_{s}^{P} \cdot\langle s, t\rangle$ se $P(t)=s \neq n i l$.

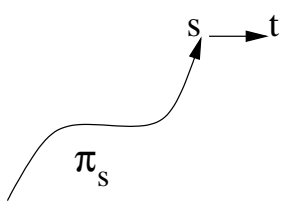

(a)

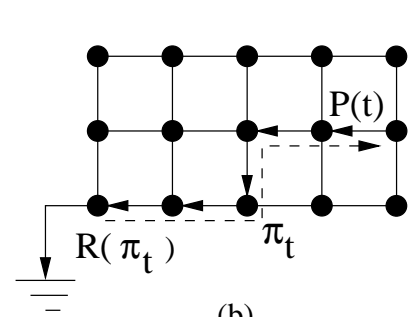

(b)

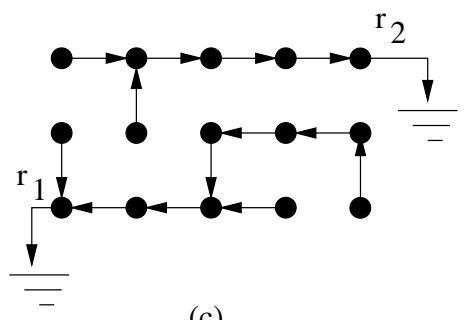

(c)

Figura 3.1: (a) Extensão de um caminho $\pi_{s}$ pela aresta $(s, t)$. (b) Grafo de vizinhança-4 mostra um caminho $\pi_{t}$ (linha pontilhada) representado no sentido inverso onde $P(t)$ é o nó predecessor de $t$ e $R\left(\pi_{t}\right)$ é o pixel raiz/origem do caminho $\pi_{t}$ (c) Floresta de espalhamento $P$ com dois nós raizes, $r_{1}$ e $r_{2}$.

\subsubsection{Função de conexidade}

Uma função de conexidade $f$ associa a qualquer caminho $\pi_{t}$ no grafo $G=(\mathcal{I}, \xi)$ um valor de custo $f\left(\pi_{t}\right)$, em algum conjunto totalmente ordenado $F$ de valores de custo, sendo que o seu inverso indica a força de conexidade entre o pixel $t$ e o nó raiz do caminho $R\left(\pi_{t}\right)$. Usualmente $f$ é baseada nos pesos das arestas que compõem o caminho.

Os exemplos mais comuns de funções de conexidade são as funções $f_{\text {sum }}$ e $f_{\max }$, as quais são definidas pelas Equações 3.1 e 3.2 respectivamente, onde $(s, t) \in \xi, \pi_{s}$ é qualquer caminho terminado em $s, H(t)$ é um custo inicial (handicap) fixo para qualquer caminho iniciando em $t$, e $\delta(s, t)$ é algum peso não negativo associado à aresta $(s, t)$.

$$
\begin{aligned}
f_{\text {sum }}(\langle t\rangle) & =H(t) \\
f_{\text {sum }}\left(\pi_{s} \cdot\langle s, t\rangle\right) & =f_{\text {sum }}\left(\pi_{s}\right)+\delta(s, t), \quad \mathrm{e} \\
f_{\max }(\langle t\rangle) & =H(t) \\
f_{\max }\left(\pi_{s} \cdot\langle s, t\rangle\right) & =\max \left\{f_{\max }\left(\pi_{s}\right), \omega(s, t)\right\}
\end{aligned}
$$

Usualmente o valor do custo inicial $H(t)$ é dado pela Equação 3.3 , onde $\mathcal{S} \subseteq \mathcal{I}$ é o conjunto de pixels sementes.

$$
H(t)= \begin{cases}0 & , \text { se } t \in \mathcal{S} \\ +\infty & , \text { caso contrário }\end{cases}
$$

De modo geral, as funções $f_{\text {sum }}$ e $f_{\text {max }}$ pertencem a uma classe de funções denominadas monotonicamente incrementais (MI), as quais satisfazem as seguintes expressões:

$$
\begin{aligned}
f_{m i}(\langle t\rangle) & =H(t) \\
f_{m i}\left(\pi_{s} \cdot\langle s, t\rangle\right) & =f_{m i}\left(\pi_{s}\right) \odot(s, t),
\end{aligned}
$$


onde $H(t)$ é arbitrário e $\odot: F \times \xi \rightarrow F$ é uma operação binária que satisfaz as condições:

(M1) $x^{\prime} \geq x \Rightarrow x^{\prime} \odot(s, t) \geq x \odot(s, t)$,

$(\mathrm{M} 2) x \odot(s, t) \geq x$

para $x, x^{\prime} \in F$ e quaisquer arestas $(s, t) \in \xi$. Uma característica essencial deste modelo de função é que $\odot$ depende apenas do valor de custo do caminho $\pi_{s}$, e não de qualquer outra propriedade deste caminho.

As funções $f_{\text {sum }}$ e $f_{\max }$ são exemplos de funções MI que podem ser usadas em operações de filtragem e segmentação de imagem, representação e descrição de objetos, e classificação de padrões usando caminhos ótimos no grafo $G$.

Alguns operadores requerem funções mais gerais que as funções MI. Este é o caso da função $f_{\text {euc }}$ usada em problemas que envolvem a transformada de distância Euclideana, a qual é definida como:

$$
\begin{aligned}
f_{\text {euc }}(\langle t\rangle) & = \begin{cases}0 & , \text { se } t \in \mathcal{S} \\
+\infty & , \text { caso contrário }\end{cases} \\
f_{\text {euc }}\left(\pi_{s} \cdot\langle s, t\rangle\right) & =\left\|t-R\left(\pi_{s}\right)\right\|^{2}
\end{aligned}
$$

onde $(s, t) \in \xi, \pi_{s}$ é qualquer caminho terminado em $s, R\left(\pi_{s}\right)$ é o pixel inicial do caminho $\pi_{s}$, e $\mathcal{S} \subseteq \mathcal{I}$ é um conjunto de sementes.

\subsubsection{Caminho ótimo}

Seja $\Pi(\mathcal{I}, \xi, t)$ o conjunto de todos os caminhos possíveis no grafo $G=(\mathcal{I}, \xi)$ com destino em $t$. Um caminho $\pi_{t}$ é ótimo se $f\left(\pi_{t}\right) \leq f\left(\pi_{t}^{\prime}\right)$ para qualquer outro caminho $\pi_{t}^{\prime} \in \Pi(\mathcal{I}, \xi, t)$, ou seja, qualquer outro caminho com o mesmo destino de $\pi_{t}$, independentemente da sua origem.

$\mathrm{O}$ valor $V(t)$ de um caminho ótimo para cada pixel $t \in \mathcal{I}$ é definido unicamente como:

$$
V(t)=\min _{\pi_{t} \in \Pi(\mathcal{I}, \xi, t)}\left\{f\left(\pi_{t}\right)\right\}
$$

A transformada imagem-floresta para um grafo $G=(\mathcal{I}, \xi)$, e para uma função de custo apropriada $f$, associa um caminho ótimo $\pi_{t}$ para cada pixel $t \in \mathcal{I}$ tal que uma floresta $P$ de caminhos ótimos é obtida (uma floresta $P$ de espalhamento onde todos os caminhos induzidos $\pi_{t}^{P}$ são ótimos). No entanto, $f$ deve ser suave, caso contrário os caminhos gerados podem não ser ótimos [Falcão et al. (2004)].

\subsubsection{Função de conexidade suave}

O artigo original da IFT [Falcão et al. (2004)] estende o algoritmo de Dijkstra para uma classe de funções mais gerais de custo, conhecidas como funções suaves, que satisfazem as seguintes condições. 
Uma função de custo $f$ é suave se para todo nó (pixel) $t \in \mathcal{I}$, existe um caminho ótimo $\pi_{t}$ que é ou trivial, ou tem a forma $\pi_{s} \cdot\langle s, t\rangle$ onde:

(C1) $f\left(\pi_{s}\right) \leq f\left(\pi_{t}\right)$

(C2) $\pi_{s}$ é ótimo,

(C3) $\pi_{s}$ é ótimo, e para qualquer caminho ótimo $\pi_{s}^{\prime}, f\left(\pi_{s}^{\prime} \cdot\langle s, t\rangle\right)=f\left(\pi_{t}\right)^{1}$.

Qualquer caminho $\pi_{t}^{P}$ induzido por uma floresta de caminhos ótimos $P$ é um caminho ótimo completo conforme à seguinte definição.

\subsubsection{Caminho ótimo completo}

Um caminho $\pi_{t_{n}}=\left\langle t_{1}, t_{2}, \ldots, t_{n}\right\rangle$ é ótimo completo se todos os caminhos $\pi_{t_{i}}=\left\langle t_{1}, t_{2}, \ldots, t_{i}\right\rangle$, para $i=1,2, \ldots, n$, são caminhos ótimos.

\subsection{Segmentação por competição de sementes}

Na segmentação via transformada imagem-floresta por competição de sementes (IFT-SC, IFT Segmentation by Seed Competition), consideramos dois conjuntos de sementes, um conjunto $\mathcal{S}_{o}$ de sementes internas composto por pixels selecionados dentro do objeto (o, object) e um conjunto $\mathcal{S}_{b}$ de sementes externas composto por pixels selecionados dentro do fundo (b, background), onde $\mathcal{S}_{o} \cap \mathcal{S}_{b}=\emptyset$. Neste paradigma, as sementes internas $\left(\mathcal{S}_{o}\right)$ e externas $\left(\mathcal{S}_{b}\right)$ competem por seus pixels mais fortemente conexos, de modo que a imagem é particionada em duas florestas de caminhos ótimos, uma enraizada nas sementes internas, definindo o objeto, e outra enraizada nas sementes externas, representando o fundo [Miranda e Falcão (2009)].

O resultado da segmentação é dada pela imagem rotulada $L$, a qual é definida por:

$$
L(t)= \begin{cases}1 & \text { se } R\left(\pi_{t}^{P}\right) \in \mathcal{S}_{o} \\ 0 & \text { caso contrário }\end{cases}
$$

onde $\pi_{t}^{P}$ é um caminho ótimo com término no pixel $t$ obtido de $P$.

Por questões de eficiência, o algoritmo da IFT (Algoritmo 1) já propaga os rótulos das raízes (sementes) para todos nós do grafo $G$, durante a sua execução.

\subsubsection{Algoritmo geral da IFT}

O algoritmo da IFT é essencialmente o procedimento de Dijkstra para o cálculo de caminhos de custo mínimo [Ahuja et al. (1993); Dijkstra (1959)], ligeiramente modificado para permitir fontes múltiplas e função mais geral de custo, isto é, obtém uma floresta de caminhos ótimos $P$ minimizando uma função de custo suave $f$.

\footnotetext{
${ }^{1}$ A condição $C 3$ apresentada corresponde à interseção das condições originais $C 2$ e $C 3$ de Falcão et al. (2004), com o fim de manter mais fácil o diagrama de classificação proposto no Capítulo 4. Note que o fato de estarmos repetindo a condição " $\pi_{s}$ é ótimo" em $C 3$ não afeta a definição de suavidade.
} 


\section{Algoritmo 1 - Algoritmo geral IFT}

Entrada: $\quad$ Imagem $\hat{I}=(\mathcal{I}, \vec{I})$, adjacência $A$, conjuntos $\mathcal{S}_{o}$ e $\mathcal{S}_{b}$ de sementes, e função de custo suave $f$.

SAídA: $\quad$ Floresta $P$ de caminhos ótimos, mapa $V$ de custos mínimos e mapa $L$ de rótulos.

Auxiliares: $\quad$ Fila $Q$ de prioridade, variável $t m p$, e um array estado do nó.

1. Para cada $t \in \mathcal{S}_{o}$, faça $L(t) \leftarrow 1$

2. Para cada $t \in \mathcal{S}_{b}$, faça $L(t) \leftarrow 0$

3. Para cada $t \in \mathcal{I}$, faça

4. $\quad P(t) \leftarrow$ nil e $V(t) \leftarrow f(\langle t\rangle)$.

5. $\quad \operatorname{estado}(t) \leftarrow 0$.

6. $\quad$ Se $V(t) \neq+\infty$, então insira t em $Q$.

7. Enquanto $Q \neq \emptyset$, faça

8. $\quad$ Remova s de $Q$ cujo valor $V(s)$ é mínimo.

9. $\quad \operatorname{estado}(s) \leftarrow 1$.

10. Para cada $t \in \mathcal{A}(s)$, tal que estado $(t)=0$, faça

11. $\quad$ Calcule tmp $\leftarrow f\left(\pi_{s}^{P} \cdot\langle s, t\rangle\right)$.

12. $\quad$ Se tmp $<V(t)$, então

13. $\quad$ Se $V(t) \neq+\infty$, então remova $t$ de $Q$.

14. $[$ Faça $P(t) \leftarrow s, V(t) \leftarrow t m p, L(t) \leftarrow L(s)$ e insira $t$ em $Q$.

O Algoritmo 1 define os rótulos como $L(t)=1$ para todo $t \in \mathcal{S}_{o}$ e $L(t)=0$ para todo $t \in \mathcal{S}_{b}$ (Linhas 1 e 2). Nas Linhas 3 a 5 , para todos os caminhos triviais inicializa os mapas de predecessores $(P)$ e de custos $(V)$, e o estado do nó. Na Linha 6 , todos os candidatos a raízes (sementes) são inseridos na fila $Q$.

O laço principal (Linhas 7 a 14) calcula os caminhos ótimos, de modo que em cada iteração um caminho $\pi_{s}^{P}$ de valor mínimo é obtido em $P$ quando removemos seu último nó $s$ de $Q$ (Linha 8). O resto de linhas (Linhas 9 a 14) calculam e propagam caminhos melhores para os nós adjacentes, seguindo uma ordem não decrescente de valores de custo e aplicando a seguinte regra de expansão: Se $f\left(\pi_{s}^{P} \cdot\langle s, t\rangle\right)<f\left(\pi_{t}^{P}\right)$ (Linha 12), então $\pi_{t}^{P}$ é trocado por $\pi_{s}^{P} \cdot\langle s, t\rangle$ (Linha 14), ou seja, o algoritmo avalia se o caminho $\pi_{s}^{P} \cdot\langle s, t\rangle$ que alcança $t$ por meio de $s$ é mais barato do que o caminho atual $\pi_{t}^{P}$ em $P$ e atualiza $P(t), V(t), L(t)$ e $Q$ adequadamente.

\subsubsection{Exemplo de segmentação}

Um exemplo de segmentação baseada em região usando a IFT é apresentado na Figura 3.2. Os marcadores $\mathcal{S}_{o}$ e $\mathcal{S}_{b}$ são colocados na imagem para indicar pixels do objeto e fundo respectivamente. A função $f_{\max }$ é escolhida com $H(t)=0$, para $t \in \mathcal{S}_{o} \bigcup \mathcal{S}_{b}, H(t)=+\infty$ caso contrário. O peso $\omega(s, t)$ das arestas é calculado de forma a ser o maior possível na borda do objeto desejado em relação aos pesos que estão dentro e fora do objeto. Os marcadores $\mathcal{S}_{o}$ e $\mathcal{S}_{b}$ competem entre si e a imagem é dividida em duas florestas de caminhos ótimos, onde o objeto é definido pela floresta de caminhos ótimos com raízes nos marcadores internos.

Na Figura 3.3 podemos observar o processo ordenado de crescimento de regiões a partir de sementes em $\mathcal{S}$ para um objeto simples usando a mesma função $f_{\max }$ e regras de inicialização. 


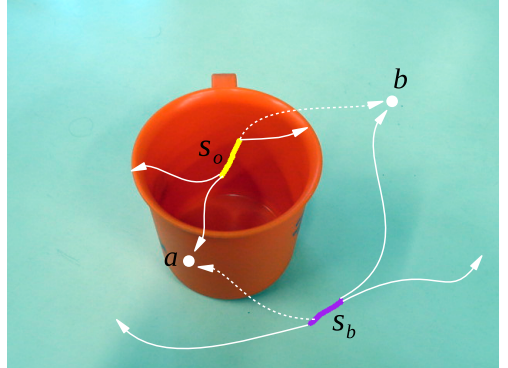

(a)

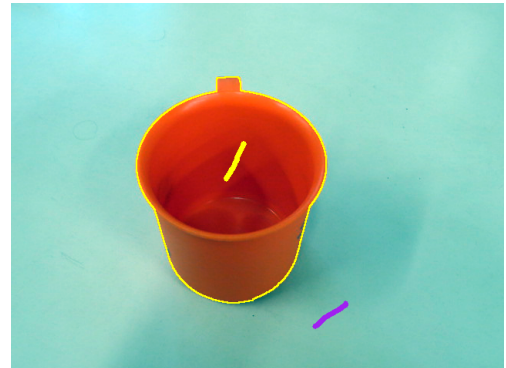

(b)

Figura 3.2: Exemplo de segmentação com dois marcadores, o marcador $\mathcal{S}_{o}$ interno representa o objeto e o marcador $\mathcal{S}_{b}$ o fundo. Em (a) são mostrados caminhos ótimos para pixels internos, como o caminho de $\mathcal{S}_{o}$ até a, e pixels externos, como o caminho de $\mathcal{S}_{b}$ até b. As linhas pontilhadas representam caminhos não ótimos. Em (b) é mostrada a borda entre as florestas ótimas interna e externa.

\subsubsection{Zonas de empate}

Na IFT, uma floresta $P$ de caminhos ótimos pode não ser única, o que representa um problema de ambiguidade das florestas ótimas [Falcão et al. (2004)]. Por exemplo, se duas ou mais raízes (sementes) alcançam um pixel $t$ por meio de caminhos ótimos com mesmo custo, então mais de uma floresta poderia ser ótima. Empates entre caminhos $\pi_{t}$ e $\pi_{t}^{\prime}$ a partir de sementes $s_{1}=R\left(\pi_{t}\right)$ e $s_{2}=R\left(\pi_{t}^{\prime}\right)$ com mesmo rótulo $\left(\left\{s_{1}, s_{2}\right\} \subset \mathcal{S}_{o}\right.$ ou $\left.\left\{s_{1}, s_{2}\right\} \subset \mathcal{S}_{b}\right)$ não representam um problema, pois eles levam ao mesmo resultado de segmentação, sendo qualquer solução satisfatória. No entanto, empates entre caminhos a partir de sementes com diferentes rótulos representam zonas de empate reais. Considerando esse último caso, podemos dizer que um pixel $t$ é um pixel de empate se existem dois caminhos ótimos completos $\pi_{t}$ e $\pi_{t}^{\prime}$ tal que $R\left(\pi_{t}\right) \in \mathcal{S}_{b}$ e $R\left(\pi_{t}^{\prime}\right) \in \mathcal{S}_{o}$. Assim, uma zona de empate é um conjunto maximal $\mathcal{T}$ de pixels de empate, o qual forma uma subárvore em alguma floresta de caminhos ótimos [Miranda e Falcão (2009)].

A Figura 3.4(d) mostra um exemplo de zonas de empate, onde os pixels de empate são aqueles dentro das regiões pontilhadas. Na Figura 3.4(b), os pixels que apresentam bordas pontilhadas são raízes de subárvores de caminhos ótimos compostas por pixels de empate. Uma forma de realizar a partição dessas regiões ambíguas é usando uma política de desempate [Falcão et al. (2004)], ou associando um rótulo fixo (1 ou 0) para todas as zonas de empate (Figuras 3.4(b) e 3.4(c)).

\subsubsection{Políticas de desempate}

O algoritmo da IFT (Algoritmo 1), na Linha 12, resolve parcialmente o problema de ambiguidade das florestas ótimas, decidindo sempre pelo primeiro caminho com menor custo que alcança um dado pixel, impedindo de atribuí-lo a qualquer outro caminho de mesmo custo que o alcance posteriormente. Assim, a única ambiguidade restante (pixels distintos alcançados por caminhos ótimos de mesmo custo) dependerá da política de desempate adotada na implementação da fila $Q$ de prioridade.

A fila $Q$ de prioridade segue uma política First-In-First-Out (FIFO), se escolhemos o primeiro pixel que entrou nela (Figuras 3.5(a) e 3.5(c)). Se escolhemos o último pixel que entrou em $Q$, nós temos uma política Last-In-First-Out (LIFO); neste caso o algoritmo também deverá selecionar sempre o último caminho ótimo encontrado, para preservar consistência no caso de empates, alterando o sinal de estritamente menor na Linha 12 para $\leq$ (Figuras 3.5(b) e 3.5(d)). 


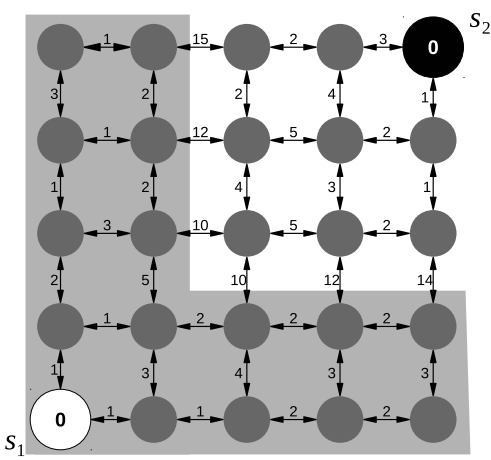

(a)

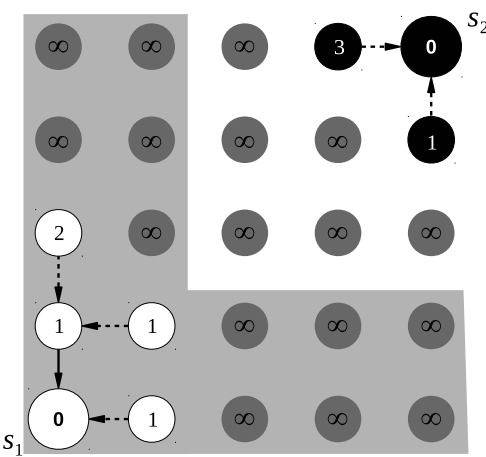

(d)

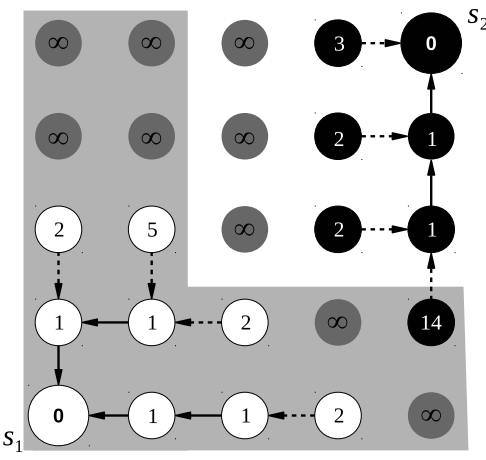

$(\mathrm{g})$

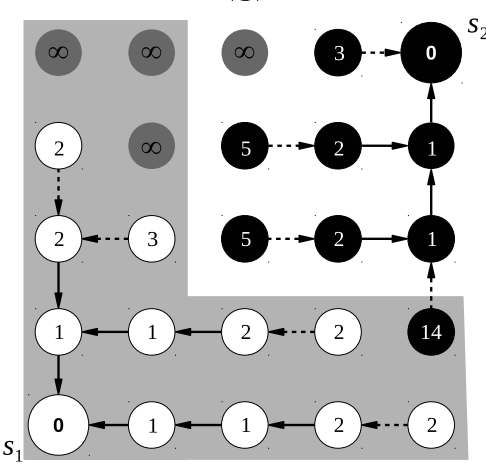

(j)

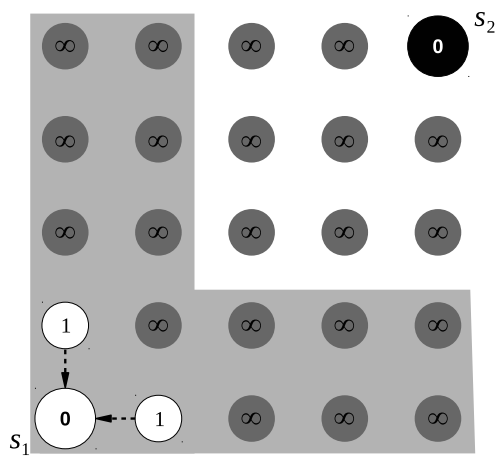

(b)

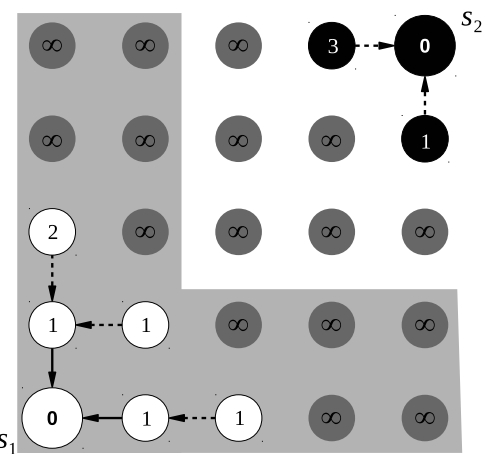

(e)

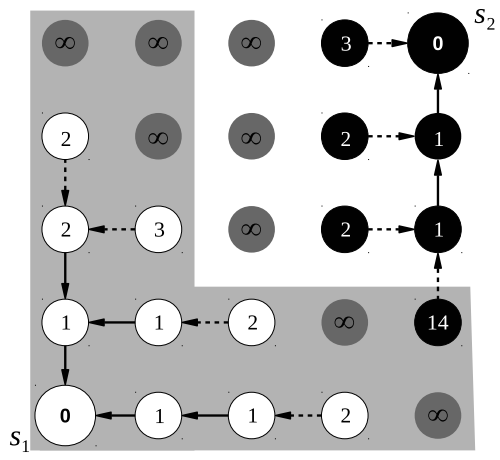

(h)

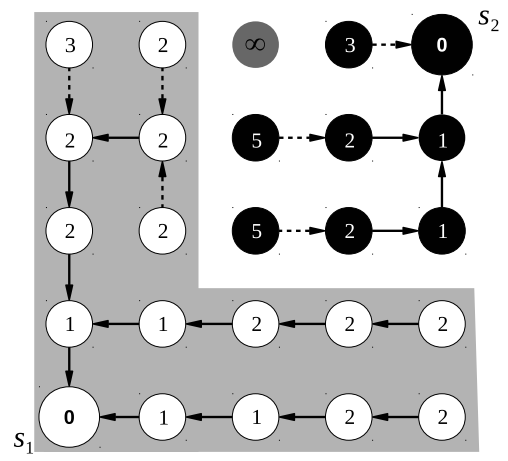

$(\mathrm{k})$

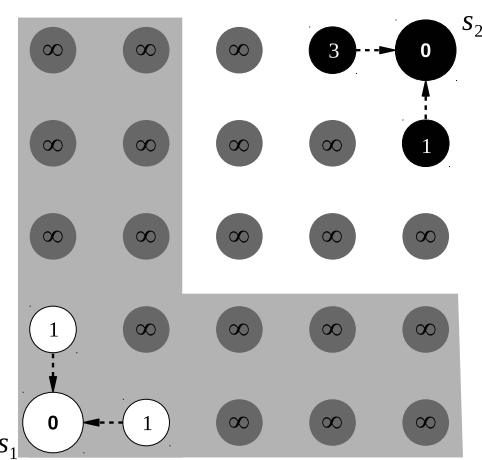

(c)

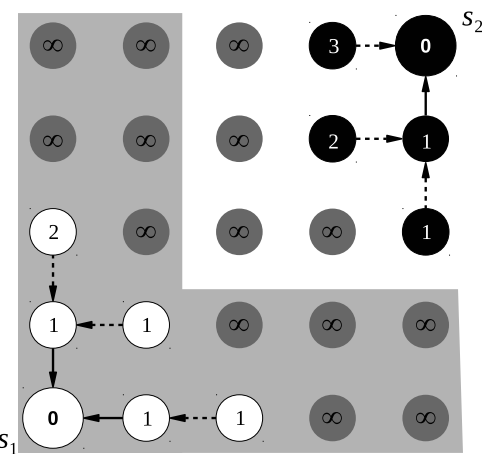

(f)

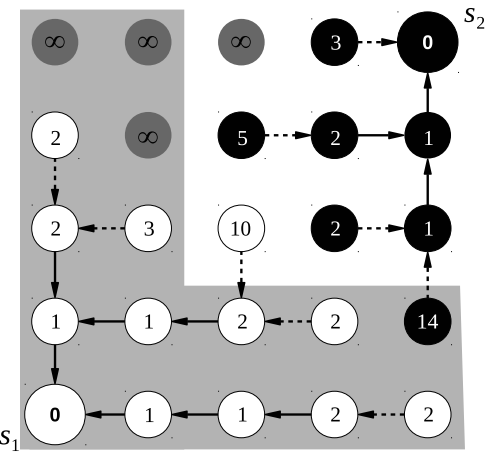

(i)

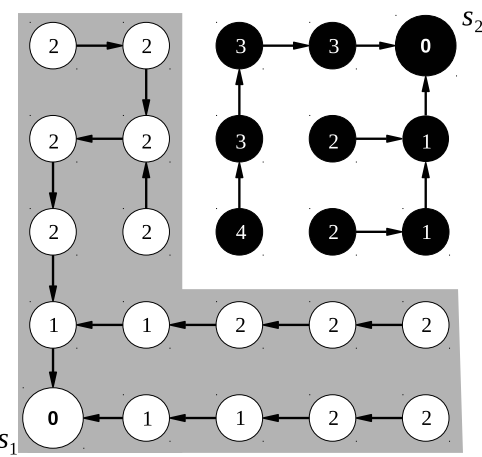

(1)

Figura 3.3: Exemplo de segmentação pela IFT com a função suave $f_{\max }$ : (a) Um grafo com adjacência 4-conexo e sementes $\mathcal{S}=\left\{s_{1}, s_{2}\right\}$. Os números exibidos dentro dos nós representam os valores do mapa $V$ de custos. (b)-(f) Floresta nas 5 primeiras iterações. (g) 8, (h) 9, (i) 12, (j) 13, (k) 18, (l) 25 iterações.

Note que na política FIFO, a zona de empate será particionada entre as árvores das raízes que a atingem, enquanto que, no caso da política LIFO, os pixels da zona de empate serão todos associados a uma mesma árvore. 


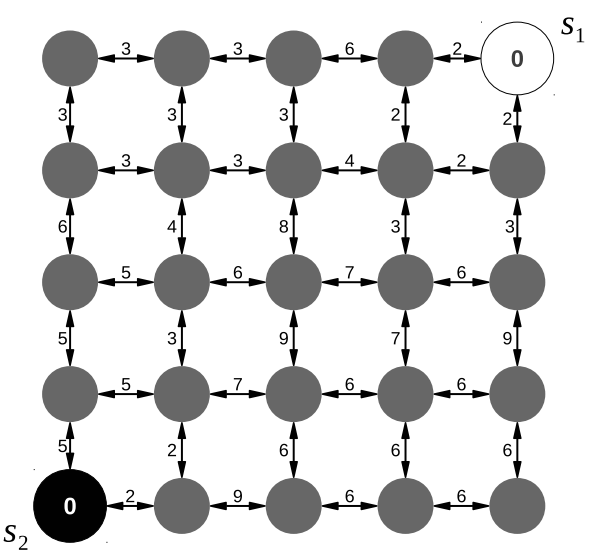

(a)

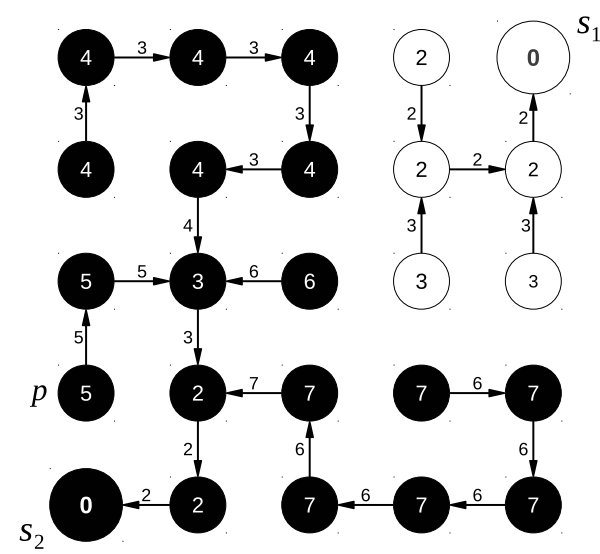

(c)

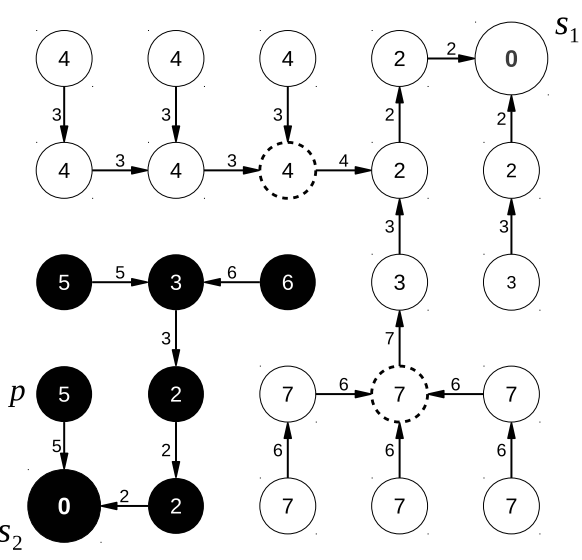

(b)

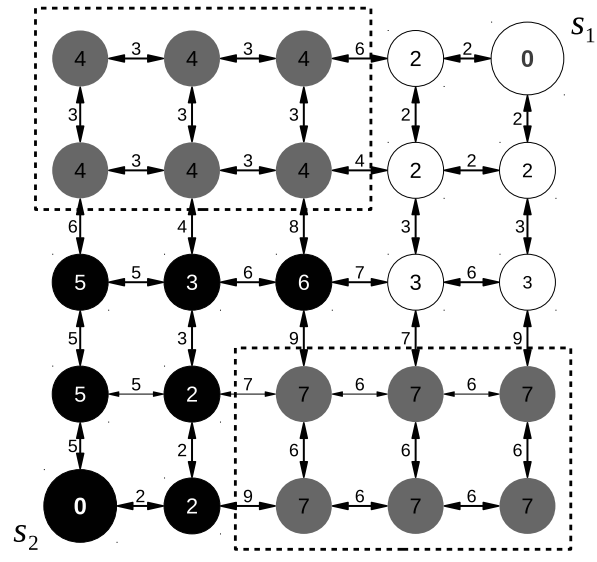

(d)

Figura 3.4: Em (a) é considerado um grafo de vizinhança-4 com uma semente que representa o objeto ( $\left.s_{1}\right)$ e uma semente representando o fundo $\left(s_{2}\right)$. Em (b) e (c) são mostradas duas possiveis florestas de caminhos ótimos usando a função de custo $f_{\max }$ (Equação 3.2). Os números dentro dos nós indicam os valores dos caminhos ótimos (Equação 3.6) Em (d) duas zonas de empate com valores de 4 e 7 são mostradas com linhas pontilhadas. O pixel p com valor 5 não é um pixel da zona de empate, pois este é rodeado sem ambiguidade por uma mesma zona de influência (preta). 


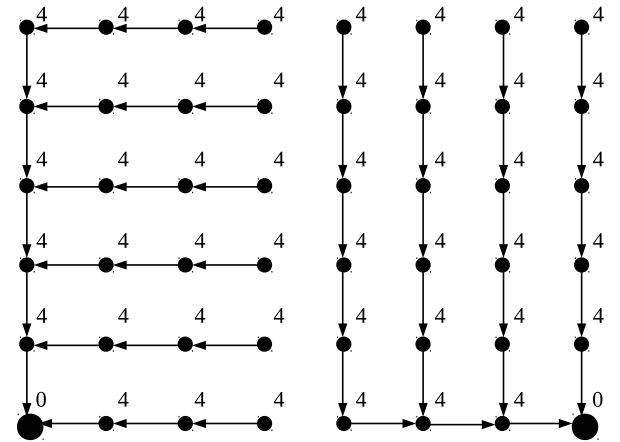

(a)

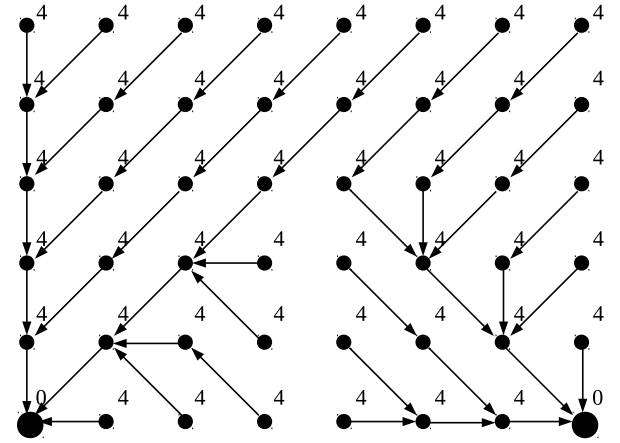

(c)

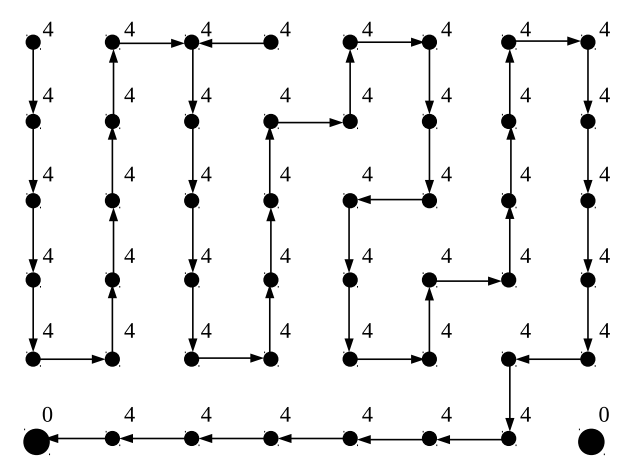

(b)

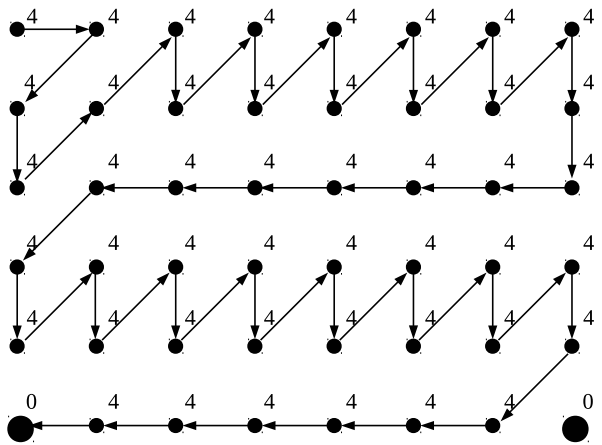

(d)

Figura 3.5: Exemplos de politicas de desempate para uma mesma função de custo, restrito a dois pixels sementes representados pelos nós maiores. (a) Política FIFO e adjacência 4-conexo. (b) Política LIFO e adjacência 4-conexo. (c) Política FIFO e adjacência 8-conexo. (d) Política LIFO e adjacência 8-conexo. 


\section{Capítulo 4}

\section{Funções de conexidade não suaves e sua classificação}

Como mencionado no Capítulo 3, o artigo original da IFT só garante resultados ótimos para o caso de funções de conexidade suaves [Falcão et al. (2004)]. No entanto, alguns outros trabalhos mostraram posteriormente que as funções de conexidade não suaves (FCNS) podem ter resultados ótimos segundo outros critérios de otimalidade [Miranda e Mansilla (2014); Miranda et al. (2012)], motivo pelo qual um estudo teórico sobre elas é importante. Nesse capítulo iniciamos a análise das funções não suaves do ponto de vista teórico e apresentamos uma classificação formal delas.

\subsection{Definição}

Como definido no capítulo anterior, uma função de conexidade é suave se cumpre as condições de suavidade $C 1, C 2$, e $C 3$ (Subseção 3.1.3); se ao menos uma delas não é satisfeita, a função é não suave.

A fim de examinar mais detalhadamente as diferentes classes de FCNS, também avaliamos as funções em relação a condição $C 4$, a qual definimos a seguir:

Definição 1 (Condição C4) Uma função de custo $f$ satisfaz a condição $C 4$. Se, para qualquer nó $s \in \mathcal{I}$, a seguinte condição é verificada $\forall t \in A(s)$ :

1. Para quaisquer caminhos $\pi_{s}$ e $\pi_{s}^{\prime}$ com destino em $s$, se $f\left(\pi_{s}\right)=f\left(\pi_{s}^{\prime}\right)$, então nós temos $f\left(\pi_{s} \cdot\langle s, t\rangle\right)=f\left(\pi_{s}^{\prime} \cdot\langle s, t\rangle\right)$.

\subsection{Classificação}

Baseados nas condições de suavidade (Subseção 3.1.3) e considerando também a nova condição $C 4$, nós avaliamos diferentes FCNS, apresentando uma classificação delas em relação as funções suaves, de acordo com o diagrama da Figura 4.1 (proposto em [Mansilla et al. (2013a)]). No Apêndice A definimos as funções $f_{\sum \max }, f_{\uparrow}, f_{\max \mid \triangle I}, f_{\sum|\triangle I|}, f_{I}$, e $f_{\sum \max }^{l e x}$; incluindo uma prova formal da classificação aqui apresentada no diagrama. As funções $f_{\max }^{b k g}, f_{\omega}, f_{\omega}^{\circlearrowleft}, f_{i, \omega}$, e $f_{o, \omega}$ foram estudadas em [Mansilla e Miranda (2013a); Miranda e Mansilla (2014); Miranda et al. (2012)]. 


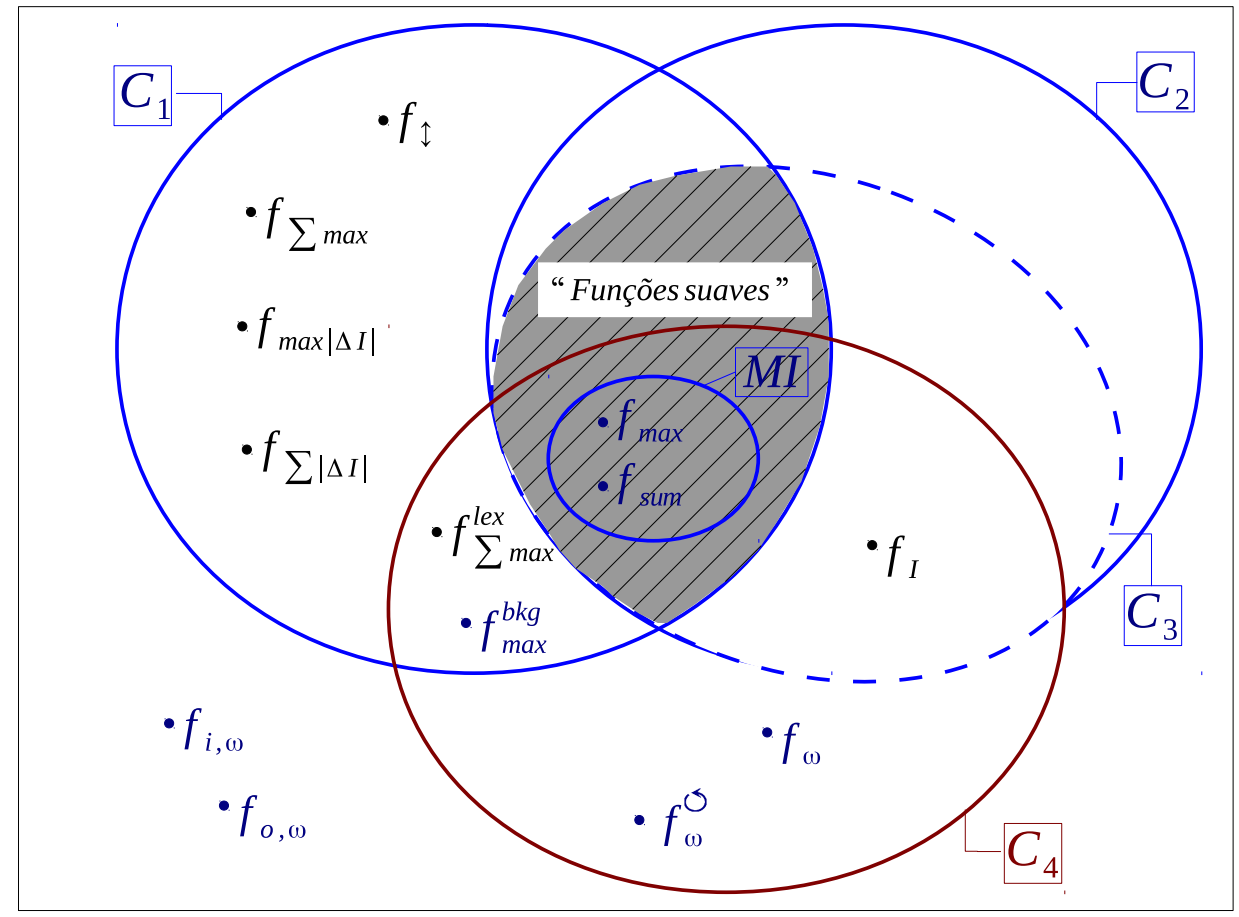

Figura 4.1: Representação esquemática das relações entre funções de conexidade suaves e não suaves: $C_{1}$, $C_{2}, C_{3}$, e $C_{4}$ são conjuntos de funções de conexidade que satisfazem suas respectivas condições. O conjunto MI representa as funções monotonicamente incrementais.

Note que algumas funções pertencentes ao subconjunto $C_{1} \backslash\left(C_{2} \bigcup C_{4}\right)$ possuem variações correspondentes na região $\left(C_{1} \cap C_{4}\right) \backslash C_{2}$ do diagrama, o que é possível através do uso de uma segunda componente de custo, com comparações em ordem lexicográfica. Por exemplo, a função lexicográfica $f_{\sum \text { max }}^{\text {lex }}$ é uma variação da função não suave $f_{\sum \text { max }}$. A região $\left(C_{1} \cap C_{4}\right) \backslash C_{2}$ será explorada em maiores detalhes no capítulo seguinte. Por outro lado, as regiões que não apresentam funções no diagrama, ainda não foram exploradas.

\subsection{Um primeiro exemplo de FCNS: Resolvendo empates da $f_{\max }$}

Como sabemos, para resolver o problema de ambiguidade de florestas ótimas podemos fazer uso das políticas de desempate apresentadas em [Falcão et al. (2004)]. No entanto, uma outra solução é incorporar uma regra de desempate dentro da própria definição da função de conexidade. Por exemplo, para resolver os empates da função $f_{\max }$ em favor do fundo, podemos considerar a função de conexidade $f_{\max }^{b k g}$ apresentada em [Miranda e Mansilla (2014)], a qual é definida como:

$$
\begin{aligned}
f_{\max }^{b k g}(\langle t\rangle) & = \begin{cases}-1 & \text { se } t \in \mathcal{S}_{o} \cup \mathcal{S}_{b} \\
+\infty & \text { caso contrário }\end{cases} \\
f_{\max }^{b k g}\left(\pi_{s} \cdot\langle s, t\rangle\right) & = \begin{cases}\max \left\{f_{\max }^{b k g}\left(\pi_{s}\right), 2 \times \omega(s, t)+1\right\} & \text { se } R\left(\pi_{s}\right) \in \mathcal{S}_{o} \\
\max \left\{f_{\max }^{b k g}\left(\pi_{s}\right), 2 \times \omega(s, t)\right\} & \text { se } R\left(\pi_{s}\right) \in \mathcal{S}_{b}\end{cases}
\end{aligned}
$$

Note que para caminhos não triviais (quando os pesos são inteiros), o custo de $f_{\text {max }}^{b k g}$ é um número ímpar para caminhos vindos de $\mathcal{S}_{o}$, e um número par para caminhos provenientes de $\mathcal{S}_{b}$, evitando assim as ambiguidades entre os diferentes rótulos, sendo a preferência dada para as sementes do 
fundo $\left(\mathcal{S}_{b}\right)$. Essa formulação, dada pela função $f_{\max }^{b k g}$, corresponde a uma nova codificação alternativa para o método Iterative Relative Fuzzy Connectedness (IRFC) [Ciesielski et al. (2011)]. Note que a função $f_{\max }^{b k g}$ é uma função não suave (Figura 4.2). Isso demonstra a importância das funções não suaves.

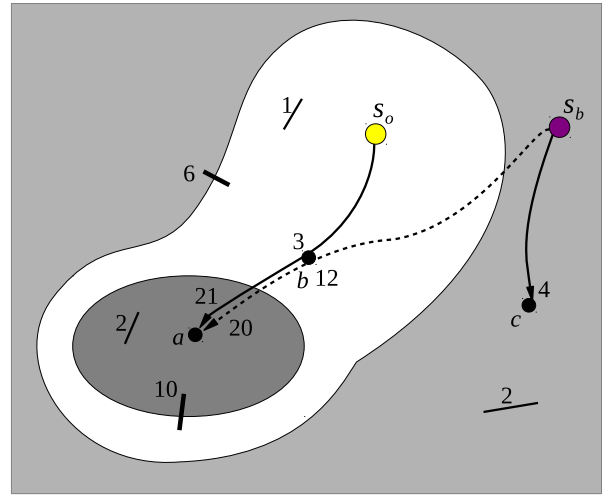

(a)

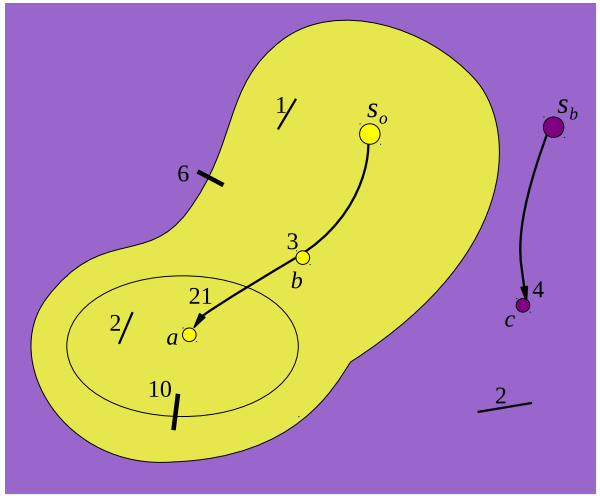

(b)

Figura 4.2: (a) Representação esquemática de um grafo onde só alguns valores representativos de aresta $\omega(s, t)$ são descritos para cada região e segmento de borda. (b) O resultado da segmentação por IFT com

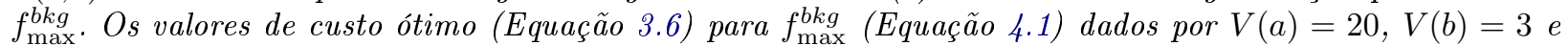
$V(c)=4$ são também indicados. Note que, para o pixel a, a semente $S_{b}$ oferece um valor de custo $V(a)=20$ que é melhor do que o custo oferecido pela semente $S_{o}$, porém o caminho vindo de $S_{b}$ não é um caminho ótimo completo, logo ele não será capaz de atingir o pixel a. Sendo assim, fbkg é uma função não suave (viola a condição C2 de suavidade).

\subsection{Função de energia (corte ótimo)}

Para o caso de grafos não direcionados com peso de aresta fixo, a IFT-SC com função $f_{\max }$ (na ausência de zonas de empate ${ }^{1}$ ) fornece resultados ótimos de segmentação segundo dois pontos de vista: Como uma floresta de caminhos ótimos [Falcão et al. (2004)], e como um corte ótimo em grafo [Ciesielski e Udupa (2011); Ciesielski et al. (2012b); Couprie et al. (2010); Miranda e Falcão (2009)].

Baseados nesse segundo enfoque temos que as funções não suaves $f_{\max }^{b k g}$ (Equação 4.1) e $f_{\omega}$ (Equação 4.2), também fornecem segmentações ótimas de acordo com uma função de energia da borda de corte [Ciesielski et al. (2012b); Couprie et al. (2010)]. As funções $f_{\max }^{b k g}$ e $f_{\omega}$ maximizam a medida de corte em grafo $E$, definida pela Equação 4.3, entre todas as possíveis segmentações satisfazendo as restrições fortes (Figura 4.3).

$$
\begin{aligned}
f_{\omega}(\langle t\rangle) & =\left\{\begin{array}{cl}
-1 & \text { se } t \in \mathcal{S}_{o} \cup \mathcal{S}_{b} \\
+\infty & , \text { caso contrário }
\end{array}\right. \\
f_{\omega}\left(\pi_{s} \cdot\langle s, t\rangle\right) & =\omega(s, t)
\end{aligned}
$$

\footnotetext{
${ }^{1}$ De um modo mais geral, podemos também considerar as condições estabelecidas em [Miranda e Falcão (2009)]
} 


$$
E(\hat{L})=\min _{(s, t) \in \xi \mid L(s) \neq L(t)} \omega(s, t)
$$

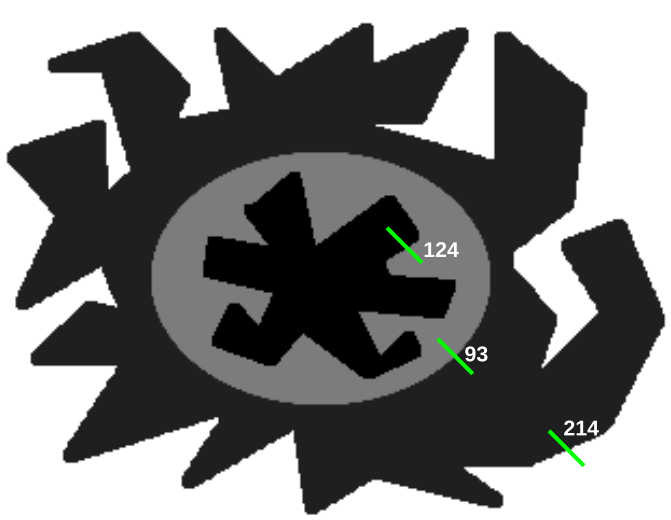

(a)

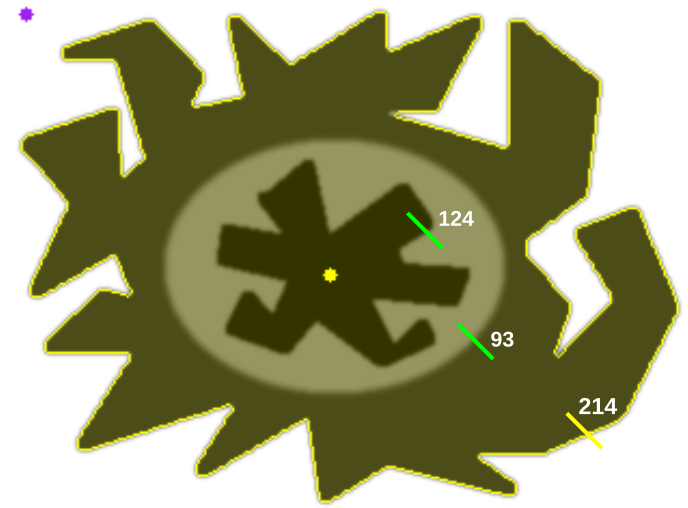

(b)

Figura 4.3: (a) Representação esquemática de três possiveis bordas de corte, alguns valores de aresta representativos $\omega(s, t)$ são representados para cada região de segmento de borda. (b) Resultado da segmentação usando a função $f_{\max }$ para as sementes mostradas. O segmento de borda com peso de aresta $\omega(s, t)$ igual a 214 tem preferência sobre os segmentos com pesos 124 e 93, devido à maximização da energia E (Equação 4.3) dada pelo minimo peso na borda de corte.

Na verdade, a otimização da energia se aplica recursivamente em cada uma das partes da borda [Miranda e Falcão (2009)], de modo que qualquer segmento de uma borda de corte é selecionado como aquele que maximiza o seu mínimo peso (Figura 4.4).

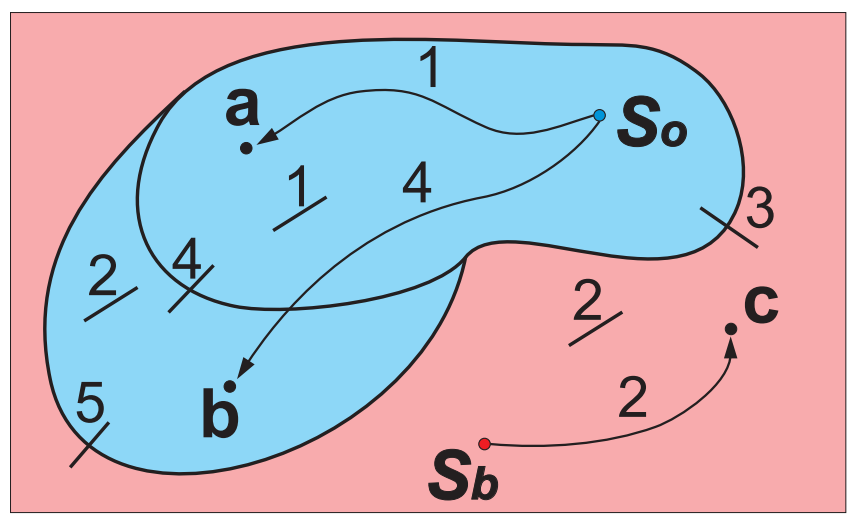

Figura 4.4: (a) Representação esquemática de um grafo onde só alguns valores de aresta representativos $\omega(s, t)$ são representados para cada região e segmento de borda. Os valores ótimos de conexidade para $f_{\max }$ (Equação 3.6) dados por $V(a)=1, V(b)=4$, e $V(c)=2$ são também indicados. O segmento de borda com pesos de aresta $\omega(s, t)$ iguais a 5 tem preferência sobre o segmento com peso 4 , devido à propriedade de otimização por partes. Note que ambas soluções levam ao mesmo corte máximo com valor mínimo $E=3$. 


\section{Capítulo 5}

\section{Funções de conexidade não suaves com pesos adaptativos}

Em análise de imagens médicas, a presença de estruturas com bordas mal definidas, a falta de padronização de intensidade entre imagens do mesmo tipo, inomogeneidade de campo, ruído, artefatos, efeitos de volume parcial e suas interações; tornam necessária a intervenção do usuário, para garantir a precisão e acurácia dos resultados. Na segmentação interativa de imagens, diferentes métodos tentam reduzir a intervenção do usuário a simples escolhas de poucos pixels na imagem.

Atualmente, métodos baseados na IFT têm sido usados com sucesso na segmentação de imagens de ressonância magnética de 1.5 Tesla. Porém, para campos magnéticos elevados, os efeitos de inomogeneidade são mais fortes (por exemplo, imagens RM de 3 Tesla) [Cappabianco et al. (2012)], motivo pelo qual é necessária a busca de soluções mais adaptativas para lidar com essas imagens com problemas de inomogeneidade.

Embora o arcabouço da IFT exija uma função de conexidade suave para garantir caminhos ótimos [Falcão et al. (2004)], neste capítulo veremos que os resultados obtidos para uma subclasse de funções de conexidade não suaves (FCNS) podem ser vistos como o resultado de uma sequência de otimizações, onde cada etapa atua sobre um conjunto maximal de elementos. Os experimentos demonstram a importância dessa classe que possui funções mais adaptativas elevando a acurácia na segmentação interativa de imagens médicas com inomogeneidade.

\subsection{Segmentação com funções de conexidade não suaves}

O diagrama de classificação das funções de conexidade (Figura 4.1), revela um subconjunto promissor de funções de conexidade não suaves $\left(f \in\left(C_{1} \cap C_{4}\right) \backslash C_{2}\right)$. Esse subconjunto apresenta algumas propriedades teóricas que podem ser reveladas pelas seguintes proposições, cujas provas formais são apresentadas no Apêndice B.

Proposição 1 Considere uma função $f \in\left(C_{1} \cap C_{4}\right) \backslash C_{2}$. Para um grafo $G=(\mathcal{I}, \xi)$ da imagem, e conjunto $\mathcal{S}$ de sementes, dados, seja $\mathcal{O}$ o conjunto de todos os pixels $t \in \mathcal{I}$, tal que existe um caminho ótimo completo $\pi_{t}$ para $f$. Em qualquer floresta de espalhamento $P$ calculada em $G$ pelo algoritmo da IFT para $f$, todos os caminhos $\tau_{t}^{P}$ com $t \in \mathcal{O}$ são caminhos ótimos.

A aplicação sucessiva da Proposição 1 dá uma caracterização do algoritmo da IFT (Algoritmo 1) 
para $f \in\left(C_{1} \cap C_{4}\right) \backslash C_{2}$, como o resultado de uma sequência de otimizações, onde cada passo de otimização envolve um conjunto maximal de elementos, de uma forma bem estruturada.

Consideremos as seguintes definições:

- Seja $\xi^{\text {set }}(X)$ o conjunto de todas as arestas interconectando nós no conjunto $X$, ou seja, $\xi^{s e t}(X)=\{(s, t) \in \xi \mid s \in X$ e $t \in X\}$

- Seja $\xi^{\text {path }}(\pi)$ o conjunto de todas as arestas no caminho $\pi$, isto é, $\xi^{\text {path }}(\pi)=\left\{\left(t_{i}, t_{i+1}\right)\right.$ para $\left.1 \leq i \leq k-1 \mid \pi=\left\langle t_{1}, t_{2}, \ldots, t_{i}, t_{i+1}, \ldots, t_{k}\right\rangle\right\}$.

- Seja $\xi^{c u t}(X, Y)$ o conjunto de todas as arestas que representam um corte no grafo, definido como $\xi^{\text {cut }}(X, Y)=\{(s, t) \in \xi \mid s \in X \wedge t \in Y\}$.

- Seja $\xi^{\text {pred }}(X)$ o conjunto de todas as arestas de caminhos induzidos na floresta $P$, com término no conjunto $X$, definido como $\xi^{\text {pred }}(X)=\bigcup_{\forall t \in X} \xi^{\text {path }}\left(\pi_{t}^{P}\right)$.

No primeiro passo da otimização, caminhos $\tau_{t}^{P}$ ótimos são calculados para todo $t \in \mathcal{O}$ (Proposição 1). Nesse primeiro passo denotamos $\mathcal{O}$ como $\mathcal{O}^{1}$.

No seguinte passo da otimização, consideramos o subgrafo $G^{2}=\left(\mathcal{I}, \xi^{\text {set }}\left(\mathcal{I} \backslash \mathcal{O}^{1}\right) \cup \xi^{c u t}\left(\mathcal{O}^{1}, \mathcal{I} \backslash \mathcal{O}^{1}\right) \cup \xi^{\text {pred }}\left(\mathcal{O}^{1}\right)\right)$. A segunda otimização de caminhos é realizada, calculando uma segunda IFT, mas agora no grafo residual $G^{2}{ }^{1}$. Uma vez que as arestas interconectando nós em $\mathcal{O}^{1}$, são reduzidas às arestas na floresta $P$ anterior $\left(\xi^{\text {pred }}\left(\mathcal{O}^{1}\right)\right)$ em $G^{2}$, nós temos que os caminhos ótimos $\tau_{t}^{P}$, calculados no passo anterior, permanecem ótimos no novo grafo $G^{2}$. Assim, os caminhos ótimos $\tau_{t}^{P} \operatorname{com} t \in \mathcal{O}^{1}$ começarão uma nova competição, em busca de suas melhores extensões para os outros pixels em $\mathcal{I} \backslash \mathcal{O}^{1}$.

Aplicando a Proposição 1 nesse novo problema de otimização uma vez mais, temos que essa segunda IFT conquistará um novo conjunto maximal de pixels $\left(\mathcal{O}^{1} \cup \mathcal{O}^{2}\right)$ que podem ser alcançados por caminhos ótimos em $G^{2}$. Então, nós podemos repetir esse processo uma vez mais. A condição $C 1^{2}$ garante que ao menos um novo elemento será conquistado a cada passo, de modo que esse processo será repetido até que $\bigcup_{\forall i} \mathcal{O}^{i}=\mathcal{I}$.

Notemos que os resultados da segmentação obtidos fazendo uso das funções pertencentes ao subconjunto $\left(C_{1} \bigcap C_{4}\right) \backslash C_{2}$, correspondem a uma sequência de otimizações em grafos residuais, o qual é similar à definição original do método Iterative Relative Fuzzy Connectedness (IRFC) [Ciesielski et al. (2007)], que foi definido como o resultado de uma sequência de otimizações pelo método Relative Fuzzy Connectedness (RFC) [Saha e Udupa (2001)].

Considerando o grafo do exemplo da Figura 3.3, na Figura 5.1 é apresentado um exemplo do processo ordenado da otimização para a função não suave $f_{\Sigma \max }^{l e x} \in\left(C_{1} \cap C_{4}\right) \backslash C_{2}$.

Proposição 2 Considere uma função de conexidade $f$, classificada como $f \in\left(C_{1} \cap C_{4}\right) \backslash C_{2}$ para um grafo geral. Se para um grafo particular $G=(\mathcal{I}, \xi)$ da imagem e conjunto $\mathcal{S}$ de sementes, dados, existe uma floresta de caminhos ótimos para $f$, entẫo qualquer floresta de espalhamento $P$ calculada em $G$ pelo algoritmo da IFT (Algoritmo 1) para $f$ é uma floresta de caminhos ótimos.

\footnotetext{
${ }^{1}$ Pelo algoritmo da IFT (Algoritmo 1), os pixels $s \in \mathcal{O}^{1}$ têm estado(s) $=1$, e seus caminhos não podem ser trocados. Portanto, nós consideramos um novo grafo $G^{2}$, onde as arestas interconectando nós em $\mathcal{O}^{1}$ e que não estão na floresta $P$ são desconsideradas.

${ }^{2}$ Por hipótese, sabemos que $f$ satisfaz a condição $C 1$.
} 


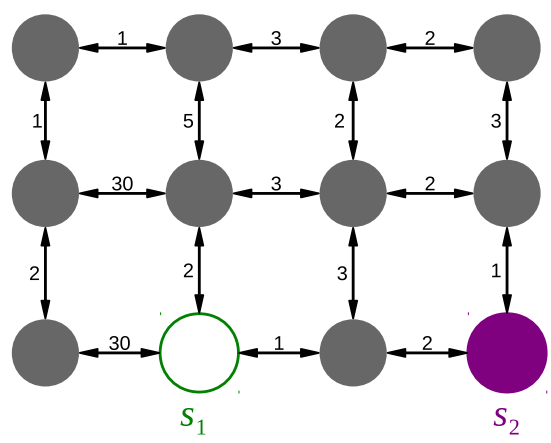

(a)

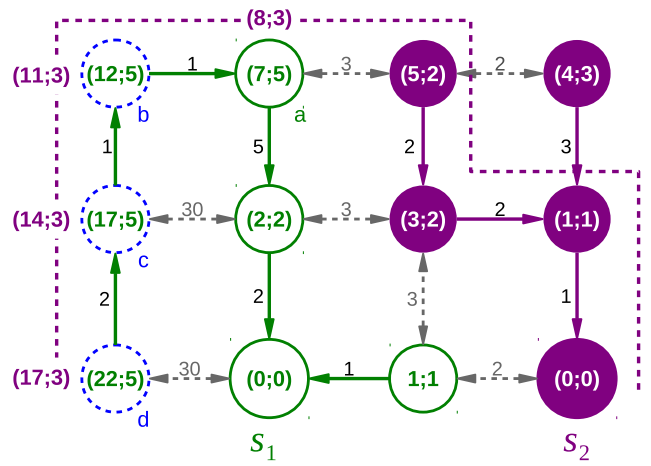

(c)

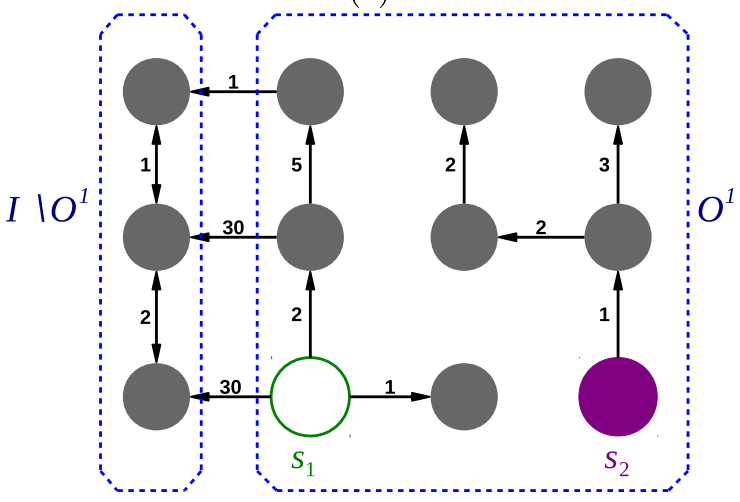

(e)

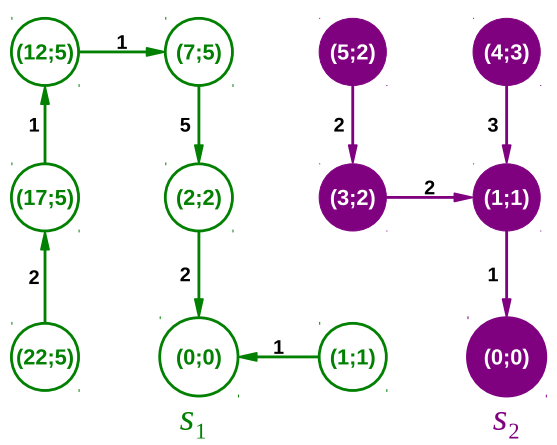

(b)

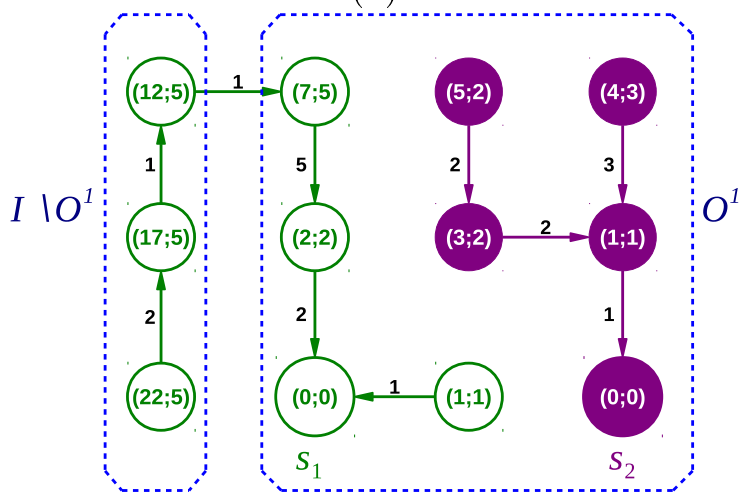

(d)

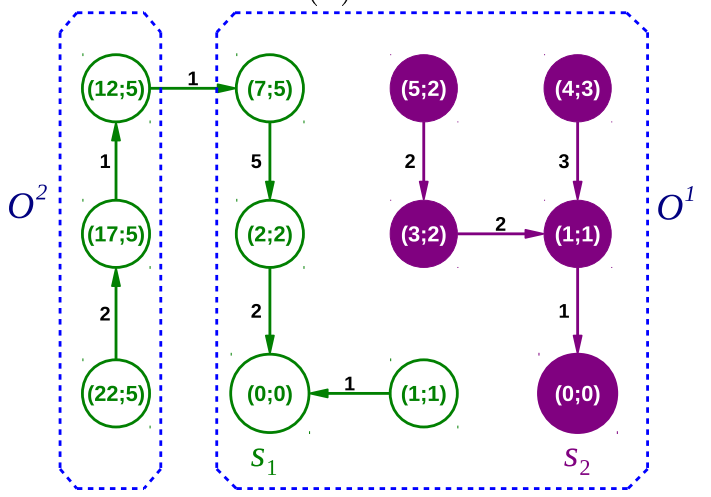

(f)

Figura 5.1: (a) Um grafo $G$ da imagem $\mathcal{I}$ com adjacência 4-conexo e sementes $\mathcal{S}=\left\{s_{1}, s_{2}\right\}$, no qual os números nas arestas representam os pesos. (b) Floresta calculada pela IFT com a função não suave $f_{\Sigma \max }^{\text {lex }} \in\left(C_{1} \cap C_{4}\right) \backslash C_{2}$. Os números dentro dos nós indicam os valores do mapa $V$ de custos com duas componentes em ordem lexicográfica. Nessa figura as setas indicam o mapa de predecessores. (c) Note que o caminho $\pi_{a}$ com destino no pixel a a partir da semente $s_{1}$ é um caminho ótimo completo, mas o caminho $\pi_{b}$ com destino no nó $b$ a partir da mesma semente não é ótimo, dado que existe outro caminho $\pi_{b}^{\prime}$ (seguindo a linha tracejada) até ele a partir da semente $s_{2}$ que oferece um melhor custo, (isto é, $f_{\Sigma \max }^{\text {lex }}\left(\pi_{b}^{\prime}\right)=(11 ; 3)<$ $\left.f_{\Sigma \max }^{\text {lex }}\left(\pi_{b}\right)=(12 ; 5)\right)$. Isso mostra que a função $f_{\Sigma \max }^{\text {lex }}$ é não suave. (d) Aplicando a Proposição 1 temos uma floresta calculada para o conjunto $\mathcal{O}^{1}$, composta por caminhos ótimos no grafo $G$ (primeira otimização). (e) Temos o grafo residual $G^{2}$, o qual será usado em uma segunda otimização. (f) Floresta ótima calculada a partir do grafo $G^{2}$, terminando o processo com $\mathcal{O}^{1} \cup \mathcal{O}^{2}=\mathcal{I}$.

Como mencionado anteriormente, o algoritmo da IFT calcula uma floresta que pode ser não ótima para FCNS, isso pode levar a pensar que existia uma tal floresta ótima, mas o algoritmo não foi capaz de encontrá-la. No entanto, como consequência da Proposição 2, temos que para qualquer função de conexidade $f \in\left(C_{1} \cap C_{4}\right) \backslash C_{2}$, o algoritmo da IFT (Algoritmo 1) somente não consegue encontrar uma floresta de caminhos ótimos, se tal floresta não existe. Portanto, não é uma falha 
do próprio algoritmo, mas sim uma falha da especificação do problema que não tem uma solução válida na forma de uma floresta de caminhos ótimos.

\subsection{Resultados experimentais para a segmentação de imagens com forte inomogeneidade}

Nos experimentos fizemos uso de dez imagens 3D de ressonância magnética de 3 Tesla, pertencentes a adultos normais, para segmentar o cérebro. Os resultados dos experimentos realizados indicam que melhoras substanciais podem ser obtidas por FCNS na segmentação 3D de imagens RM de 3 Tesla, em comparação com as funções de conexidade suaves. As curvas de acurácia são apresentadas na Figura 5.2, onde as sementes foram obtidas por um usuário robô, segundo o procedimento introduzido por Gulshan et al. [Gulshan et al. (2010)]. Em particular a função $f_{\sum|\triangle I|}^{l e x} \in\left(C_{1} \cap C_{4}\right) \backslash C_{2}$ demonstrou resultados muito bons para a segmentação 3D de imagens RM de 3 Tesla.

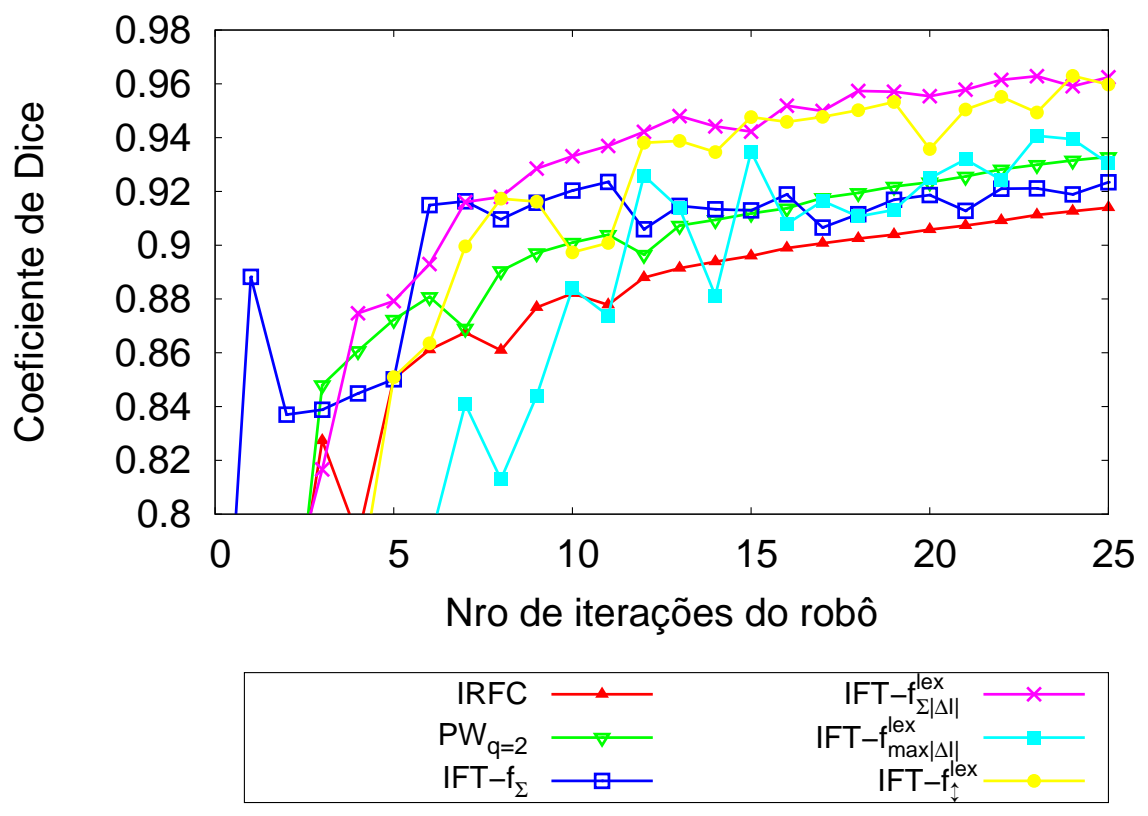

Figura 5.2: Resultados usando um usuário robô para a segmentação do conjunto de dados do cérebro em $3 D$.

Um exemplo de segmentação 3D para marcadores selecionados pelo usuário é mostrado na Figura 5.3, cujos resultados obtidos permitem observar a importância das funções não suaves.

Embora os resultados obtidos pela função suave $f_{\max }$ (por exemplo, IRFC) possam ser melhorados através do uso de técnicas de realce das bordas do objeto [Miranda et al. (2010)], isso exigiria um passo de treinamento indesejável. Na Figura 5.4 podemos observar que a IFT com $f_{\sum|\triangle I|}^{l e x}$ não só evita qualquer fase de treinamento, como também pode lidar melhor com os efeitos da inomogeneidade.

\footnotetext{
${ }^{3}$ A função $f_{\sum|\triangle I|}^{l e x}$ fornece pares de valores que devem ser comparados de acordo com uma ordem lexicográfica, o primeiro valor é dado pela função não suave $f_{\sum|\triangle I|}$ (Equação A.6), e o segundo é o nível de prioridade da semente para esse caminho, sendo que quanto menor o valor maior é a sua prioridade. Na segmentação interativa, damos prioridade mais baixa para novas sementes inseridas de modo a manter seus efeitos mais localmente, pois elas são usadas somente para corrigir erros de segmentação.
} 


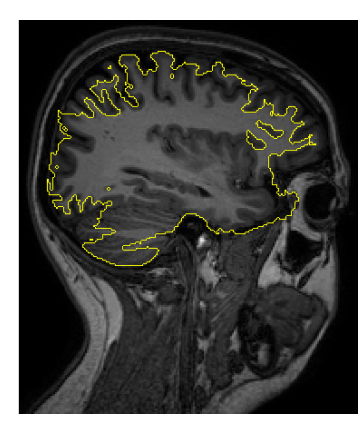

(a)

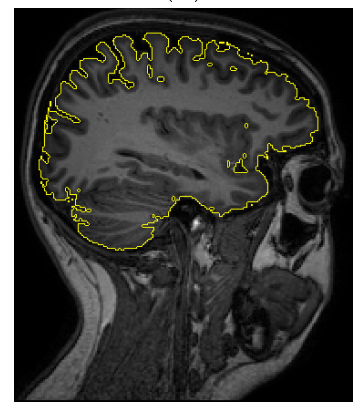

(e)

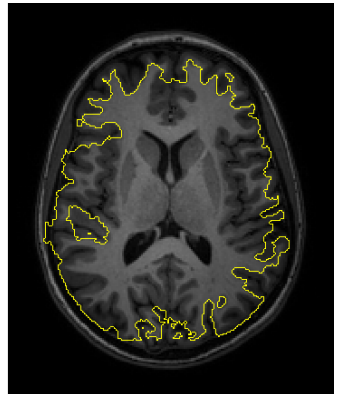

(b)

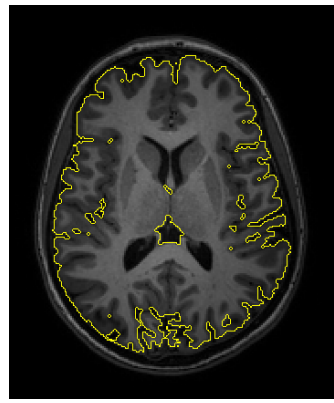

(f)

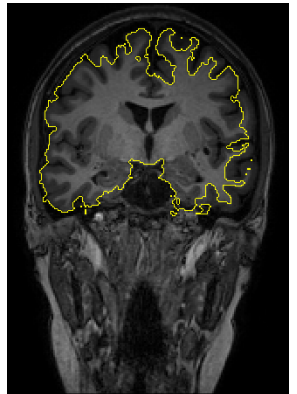

(c)

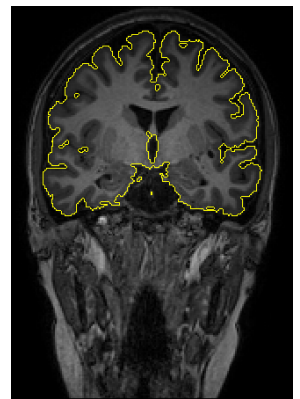

(g)
INOMOGENEIDADE $\quad 37$

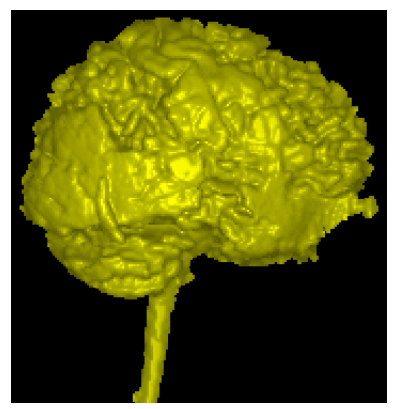

(d)

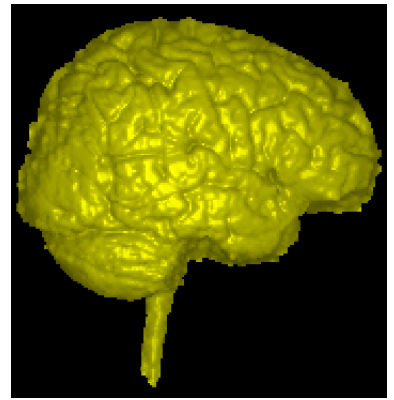

(h)

Figura 5.3: Resultado da segmentação $3 D$ do cérebro para os mesmos marcadores selecionados pelo usuário para as funções: (a)-(d) $f_{\max }, e(e)-(h) f_{\sum|\Delta I|}^{\text {lex }}$.

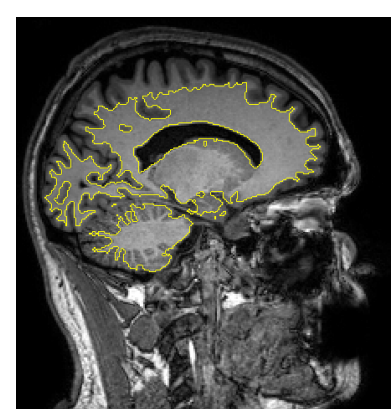

(a)

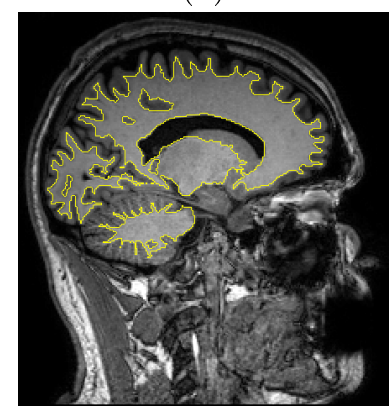

(e)

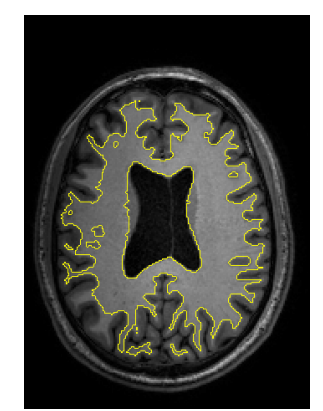

(b)

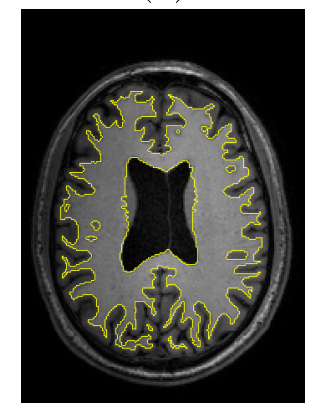

(f)

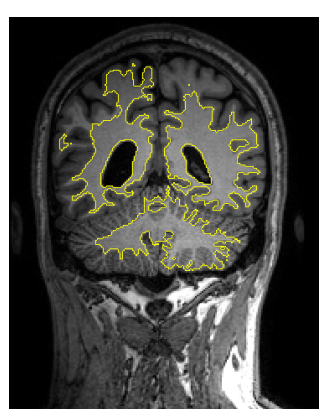

(c)

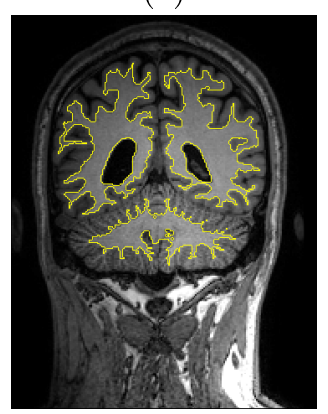

(g)

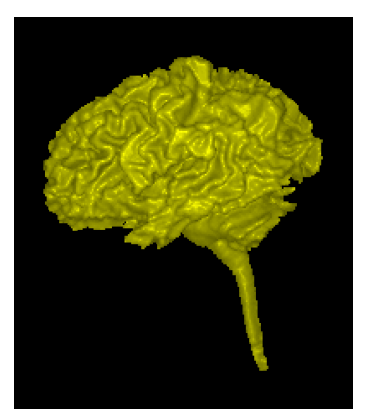

(d)

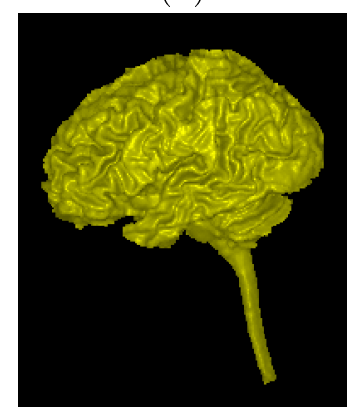

(h)

Figura 5.4: Segmentação $3 D$ da substância branca para os mesmos marcadores selecionados pelo usuário. Resultados obtidos pelas funções (a)- $(d) f_{\max }$ sobre um gradiente realçado, e $(e)-(h) f_{\sum|\triangle I|}^{\text {lex }}$.

Na Figura 5.5 é mostrado um exemplo de realce do gradiente de uma imagem com problemas de inomogeneidade, onde o objeto a realçar é a substância branca. Nessa figura podemos observar que para marcadores localizados em regiões claras do objeto (Figura 5.5(a)) o mapa de pertinência via classificação supervisionada "fuzzy" resultante apresenta perdas de informação (Figura 5.5(c)), 


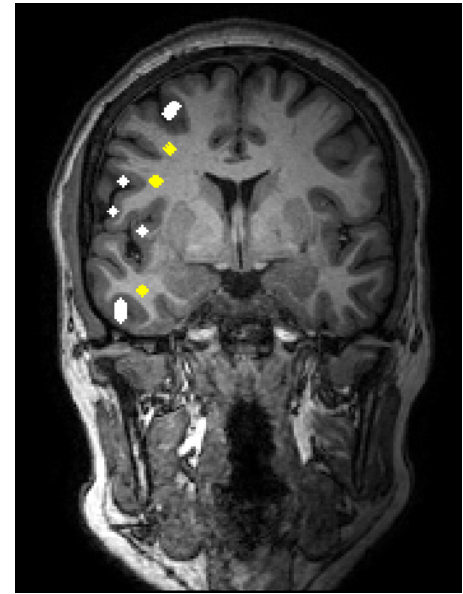

(a)

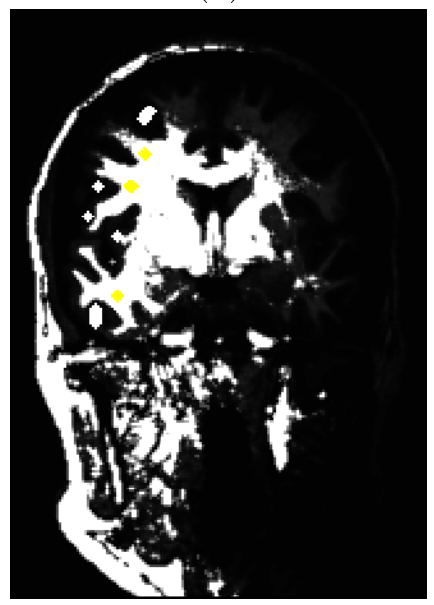

(c)

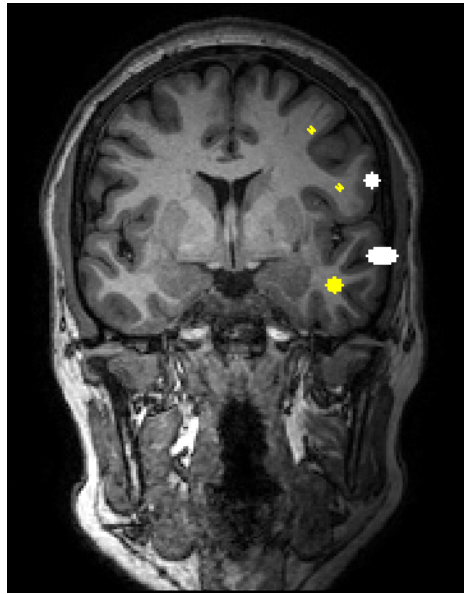

(b)

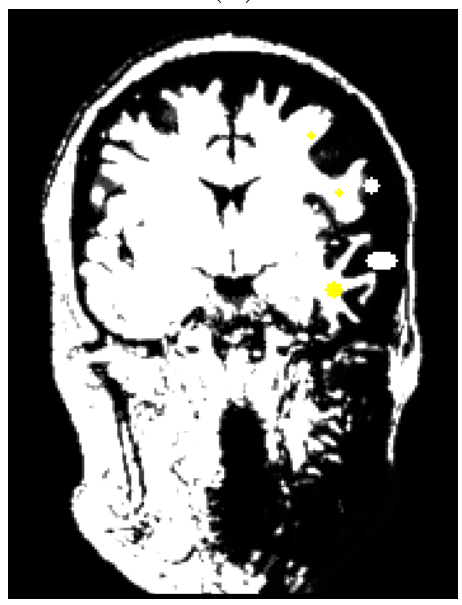

(d)

Figura 5.5: Exemplo de realce de bordas em uma imagem com problemas de inomogeneidade. Os marcadores amarelos, representando o objeto, indicam a substância branca e os marcadores brancos representam o fundo. Usando as técnicas de realce das bordas do objeto apresentadas em [Miranda et al. (2010)], obtemos os mapas de pertinência (c) e (d), para marcadores localizados no hemisfério (a) esquerdo e (b) direito, respectivamente.

e para marcadores em regiões mais escuras (Figura 5.5(b)) a classificação resultante considera como parte do objeto outras regiões correspondentes a diferentes tecidos (Figura 5.5(d)). Sendo assim, técnicas tradicionais de realce, comumente usadas em artigos (Seção 1.1), não proporcionam uma solução adequada na segmentação de imagens com problemas de inomogeneidade. 


\section{Capítulo 6}

\section{Funções de conexidade não suaves para segmentação com polaridade de borda}

Estruturas fracamente definidas tanto em imagens médicas, bem como em imagens naturais, têm sido objeto de estudo em processamento de imagens e visão computacional. Assim, diferentes métodos de segmentação semi-automática foram desenvolvidos com o fim de reduzir as intervenções do usuário [Boykov e Funka-Lea (2006); Falcão et al. (1998); Mortensen e Barrett (1998); Saha e Udupa (2001)]. Alguns métodos baseados em borda (por exemplo, live wire [Falcão et al. (1998); Mortensen e Barrett (1998)]) exploram a orientação da borda esperada, para resolver ambiguidades entre segmentos de borda semelhantes muito próximos (Figura 6.1), favorecendo a segmentação em uma única direção (horária ou anti-horária).

Neste capítulo, nós apresentamos uma extensão da transformada imagem-floresta por competição de sementes (IFT-SC, IFT segmentation by Seed Competition) [Miranda e Falcão (2009)] que explora a informação sobre a orientação da borda dos objetos, em grafos direcionados, mediante o uso de novas funções de conexidade não suaves. Os resultados são suportados teoricamente com base em um critério de otimalidade de corte em grafo.

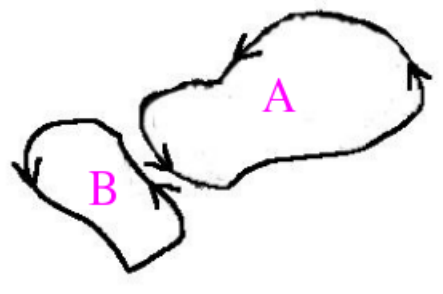

(a)

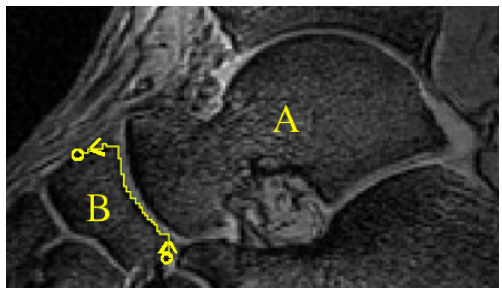

(b)

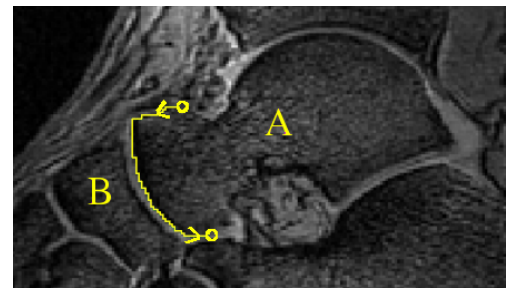

(c)

Figura 6.1: Exemplo de segmentação baseada em borda, onde a propriedade de orientação de borda do live wire o ajuda a distinguir entre dois segmentos de borda similares [Falcão et al. (2000)].

\subsection{Transformada imagem-floresta orientada (OIFT)}

Na Seção 4.4, vimos que as funções de conexidade $f_{\max }, f_{\max }^{b k g}$ e $f_{\omega}$ permitem uma solução ótima global de acordo com uma função de energia em grafos não direcionados (Equação 4.3), a qual obtém um corte ótimo na segmentação pelo algoritmo da IFT (Algoritmo 1). Para o caso de grafos 
direcionados consideramos dois tipos de corte na borda do objeto a segmentar, um corte interno e um corte externo. Os cortes internos (inner cut) são compostos por arestas que apontam em direção aos pixels do objeto (Figura 6.2(a)), e os cortes externos (outer cut) por arestas direcionadas do objeto para os pixels do fundo (Figura 6.2(b)).

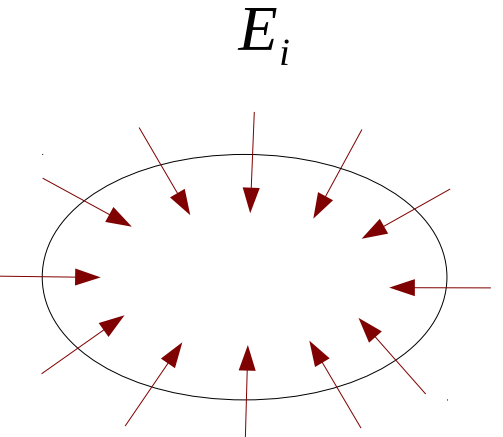

(a)

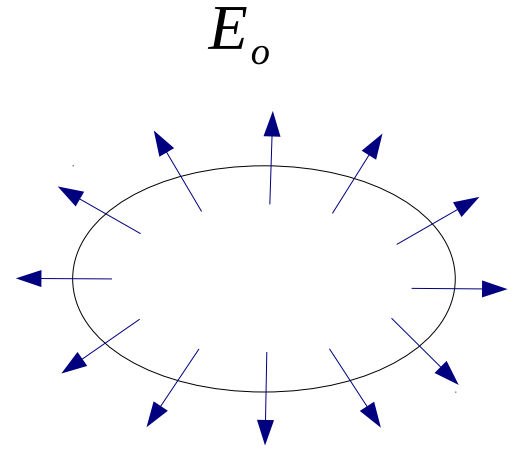

(b)

Figura 6.2: Em (a) considera-se uma energia interna $E_{i}$ para os cortes internos na borda do objeto. Em (b) uma energia externa $E_{o}$ é considerada para os cortes externos na mesma borda.

Cada tipo de corte possui sua própria definição de energia, de modo que consideraremos uma função de energia interna $E_{i}$ (Equação 6.1) dada pelo mínimo dos cortes internos na borda, e uma função de energia externa $E_{o}$ (Equação 6.2) para os cortes externos.

$$
\begin{aligned}
& E_{i}(\hat{L})=\min _{(s, t) \in \xi \mid L(s)=0, L(t)=1} \omega(s, t) \\
& E_{o}(\hat{L})=\min _{(s, t) \in \xi \mid L(s)=1, L(t)=0} \omega(s, t)
\end{aligned}
$$

Para os valores dos pesos das arestas $\omega(p, q)$ do dígrafo consideramos uma configuração definida por uma combinação de uma medida regular de dissimilaridade $\psi(p, q)$ multiplicada por um fator de orientação $(1+\alpha$ ou $1-\alpha)$. Essa configuração é definida na Equação 6.3. Ela favorece a segmentação de imagens cujo objeto apresente na sua borda transições de pixels de escuro para claro considerando cortes internos (Figura 6.3(a)) ou de claro para escuro considerando cortes externos (Figura 6.3(b)).

$$
\omega(p, q)= \begin{cases}\psi(p, q) \times(1+\alpha) & \text { se } I(p)>I(q) \\ \psi(p, q) \times(1-\alpha) & \text { se } I(p)<I(q) \\ \psi(p, q) & \text { caso contrário. }\end{cases}
$$

Em geral consideramos $\psi(p, q)$ igual ao valor absoluto da diferença de intensidades dos pixels $p$ e $q$ na imagem (isto é, $\psi(p, q)=|I(p)-I(q)|$ ). Note que temos um grafo ponderado direcionado $(\omega(p, q) \neq \omega(q, p))$ quando $\alpha>0$. 


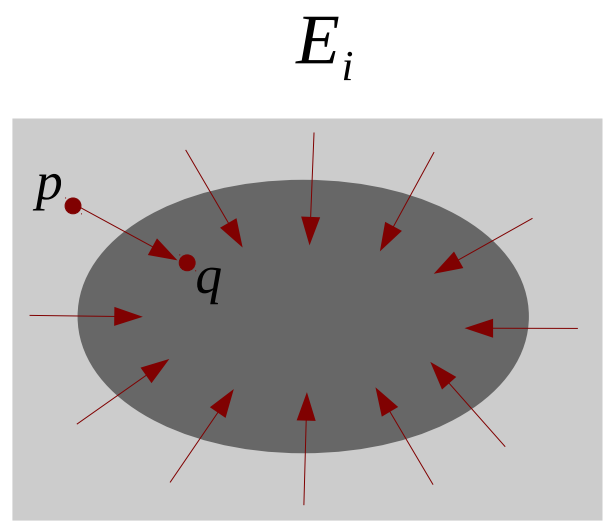

(a)

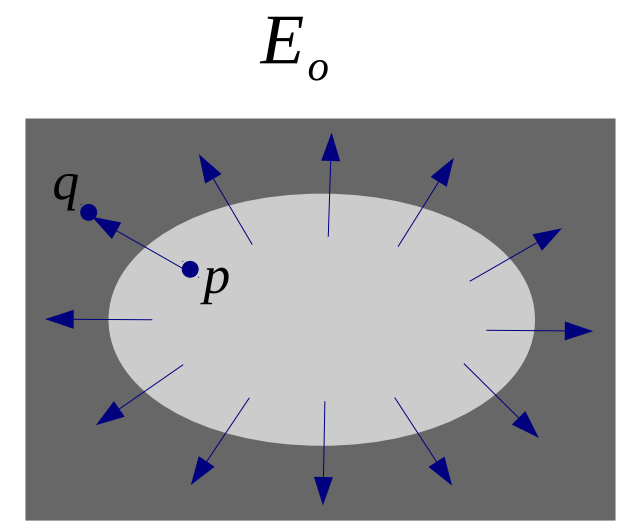

(b)

Figura 6.3: Em (a) temos uma transição de pixels de escuro para claro na borda do objeto com cortes internos $(p, q) \in \xi$. Em (b) temos uma borda com transições de pixels de claro pra escuro considerando cortes externos $(p, q) \in \xi$.

\subsection{Segmentação pela OIFT com as funções de conexidade não su- aves $f_{i, \max }^{b k g}$ e $f_{o, \max }^{b k g}$}

Para garantir a otimalidade dos resultados do ponto de vista de energias nas bordas de corte, precisamos modificar as funções de custo. Nós modificamos a função $f_{\max }^{b k g}$ obtendo as funções $f_{i, \max }^{b k g}$ e $f_{o, \max }^{b k g}$, definidas por:

$$
\begin{aligned}
& f_{i, \text { max }}^{b k g}(\langle t\rangle)=\left\{\begin{array}{lll}
-1 & \text { se } t \in \mathcal{S}_{o} \cup \mathcal{S}_{b} \\
+\infty & \text { caso contrário }
\end{array}\right. \\
& f_{i, \text { max }}^{b k g}\left(\pi_{s} \cdot\langle s, t\rangle\right)= \begin{cases}\max \left\{f_{i, \max }^{b k g}\left(\pi_{s}\right), 2 \times \omega(t, s)+1\right\} & \text { se } R\left(\pi_{s}\right) \in \mathcal{S}_{o} \\
\max \left\{f_{i, \max }^{b k g}\left(\pi_{s}\right), 2 \times \omega(s, t)\right\} & \text { se } R\left(\pi_{s}\right) \in \mathcal{S}_{b}\end{cases} \\
& f_{o, \text { max }}^{b k g}(\langle t\rangle)=\left\{\begin{array}{lll}
-1 & \text { se } t \in \mathcal{S}_{o} \cup \mathcal{S}_{b} \\
+\infty & \text { caso contrário }
\end{array}\right. \\
& f_{o, \text { max }}^{b k g}\left(\pi_{s} \cdot\langle s, t\rangle\right)= \begin{cases}\max \left\{f_{o, \text { max }}^{b k g}\left(\pi_{s}\right), 2 \times \omega(s, t)+1\right\} & \text { se } R\left(\pi_{s}\right) \in \mathcal{S}_{o} \\
\max \left\{f_{o, \max }^{b k g}\left(\pi_{s}\right), 2 \times \omega(t, s)\right\} & \text { se } R\left(\pi_{s}\right) \in \mathcal{S}_{b}\end{cases}
\end{aligned}
$$

As funções $f_{i, \max }^{b k g}$ e $f_{o, \text { max }}^{b k g}$ otimizam energias de cortes internos e externos, isto é, cortes ótimos que maximizam as Equações 6.1 e 6.2 respectivamente. Na Figura 6.4 é exibido um exemplo que mostra que $f_{o, \max }^{b k g}$ é uma função não suave.

Como temos mencionado anteriormente, no caso de FCNS, a IFT retornará uma floresta de espalhamento, mas os caminhos podem não ser ótimos [Falcão et al. (2004)]. Contudo, isso não afetará o resultado ótimo do ponto de vista das energias $E_{i}(\hat{L})$ e $E_{o}(\hat{L})$, para as funções $f_{i, \max }^{b k g}$ e $f_{o, \max }^{b k g}$, respectivamente, como provado no Apêndice C pelos Teoremas 1 e 2. 


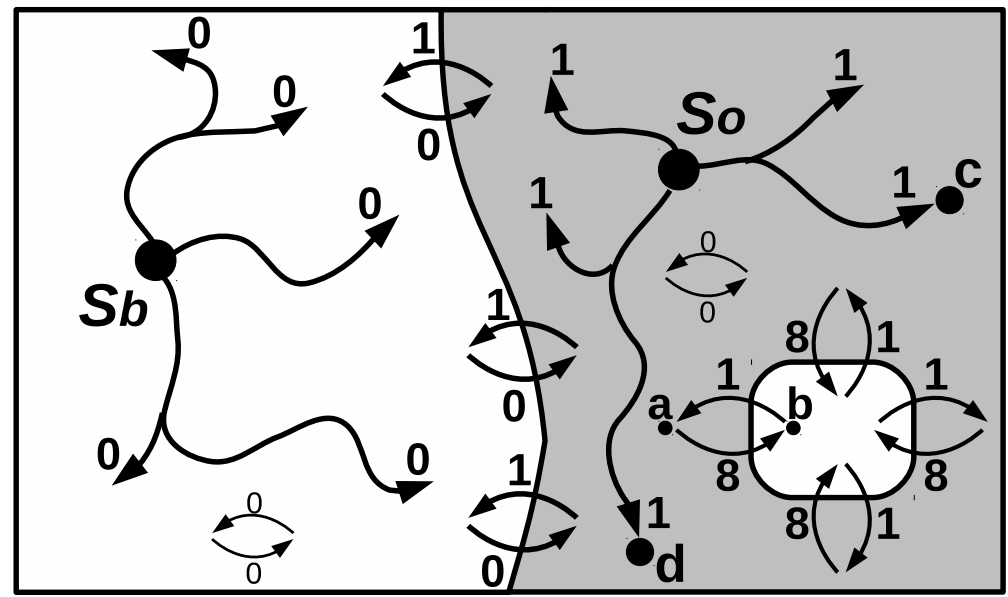

Figura 6.4: A função $f_{o, \max }^{b k g}$ faz uso de arestas invertidas para caminhos a partir de $\mathcal{S}_{b}$. Suponhamos que $\mathcal{S}_{b}$ conquista a região branca da esquerda com custo 0 , dado que seus pixels são interconectados por arestas com peso 0 . Os pixels dentro da região cinza tem também arestas com peso 0 , assim $\mathcal{S}_{o}$ fornece custos melhores a eles $\left(2 \times 0+1=1\right.$ contra $2 \times 1=2$ oferecido por $\left.\mathcal{S}_{b}\right)$. O melhor custo oferecido por $\mathcal{S}_{o}$ ao nó b é $2 \times 8+1=17$, enquanto que $\mathcal{S}_{b}$ poderia ter fornecido um melhor custo a b $(2 \times 1=2)$, mas b é atribuído ao objeto. Portanto, a função não é suave.

O Teorema 1, fornece uma condição suficiente para soluções ótimas dos cortes $E_{i}(\hat{L})$ e $E_{o}(\hat{L})$, respectivamente.

Teorema 1 (Otimalidade dos cortes internos/externos) Para um grafo $G=(\mathcal{I}, \xi)$ da imagem, e dois conjuntos $\mathcal{S}_{o}$ e $\mathcal{S}_{b}$ de sementes, se existe uma floresta de caminhos ótimos para a função $f_{o, \max }^{b k g}$, então seu corte é um corte ótimo que maximiza $E_{o}(\hat{L})$ entre todos os possíveis resultados da segmentação satisfazendo as restriçôes fortes. Se existe uma floresta de caminhos ótimos para a função $f_{i, \max }^{b k g}$, então seu corte é um corte ótimo que maximiza $E_{i}(\hat{L})$ entre todos os possíveis resultados da segmentação satisfazendo as restrições fortes.

A seguir, o Teorema 2 estende esses resultados para o caso geral sem a necessidade da suposição da otimalidade dos caminhos.

Teorema 2 (Otimalidade dos cortes internos/externos - caso geral) Para dois conjuntos $\mathcal{S}_{o}$ e $\mathcal{S}_{b}$ de sementes dados, qualquer floresta de espalhamento calculada pelo algoritmo da IFT (Algoritmo 1) para a função $f_{o, \max }^{b k g}$ define um corte ótimo que maximiza $E_{o}(\hat{L})$ entre todos os resultados possiveis da segmentação satisfazendo as restrições fortes. Qualquer floresta de espalhamento calculada pelo algoritmo da IFT (Algoritmo 1) para a função $f_{i, \max }^{b k g}$ define um corte ótimo que maximiza $E_{i}(\hat{L})$ entre todos os resultados possiveis da segmentação satisfazendo as restrições fortes.

\subsubsection{Resultados experimentais}

Nos experimentos, nós consideramos os pesos das arestas $\omega(p, q)$ segundo a configuração da Equação 6.3. Nós usamos $\alpha=0.5$ em todos os experimentos $2 \mathrm{D}$ envolvendo $f_{o, \max }^{b k g}$ e $f_{i, \max }^{b k g}$, e $\alpha=0.0$ no caso do enfoque não direcionado $f_{\max }^{b k g}$.

Para a nossa configuração dos pesos do grafo, a função $f_{i \text { max }}^{b k g}$ favorece a segmentação de objetos com transições de pixels escuros para claros nas bordas, enquanto a função $f_{o \text {,max dá preferência às }}^{b k g}$ 
transições de claro para escuro. Isto é, quando a borda do objeto a ser segmentado apresenta uma transição de pixels de escuro para claro, a IFT com $f_{i, \max }^{b k g}$ melhora os resultados em relação a $f_{\max }^{b k g}$, enquanto que a IFT com $f_{o, \max }^{b k g}$ piora os resultados, por considerar a orientação de borda errada; o oposto acontecerá no caso inverso.

Nas Figuras 6.5 e 6.6 mostramos os resultados da segmentação dos ossos do pé tálus e calcâneo respectivamente, com a função $f_{i, \max }^{b k g}$. Na Figura 6.7 podemos observar o resultado da segmentação da coluna vertebral considerando a função $f_{o, \max }^{b k g}$ por apresentar uma transição de pixels de claro para escuro.

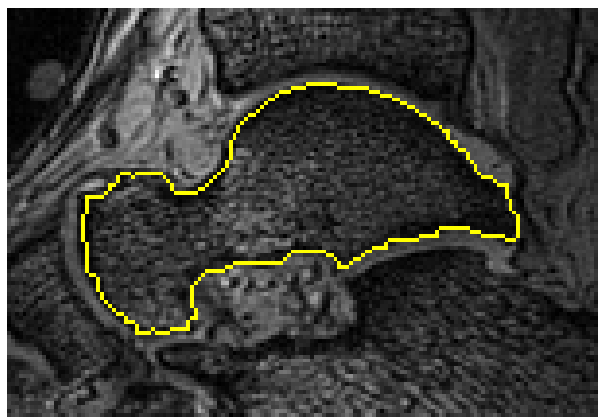

(a)

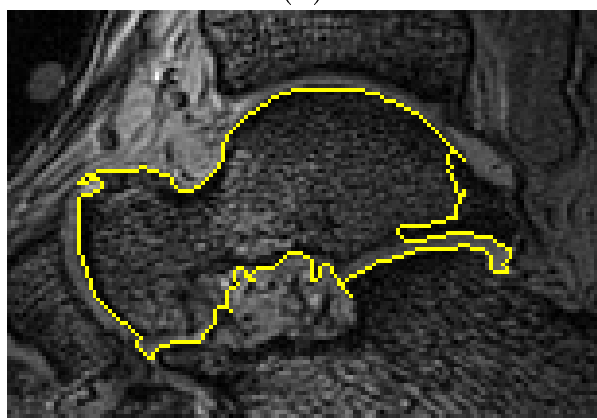

(c)

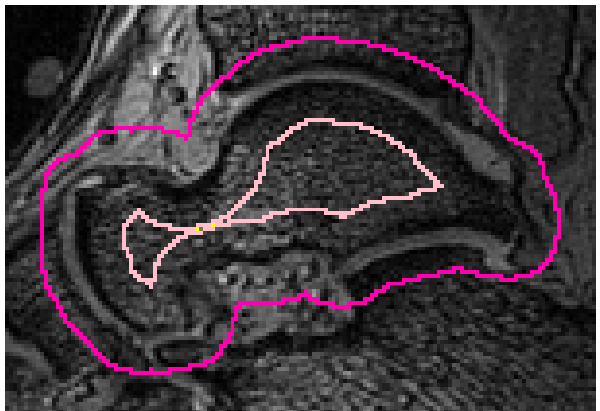

(b)

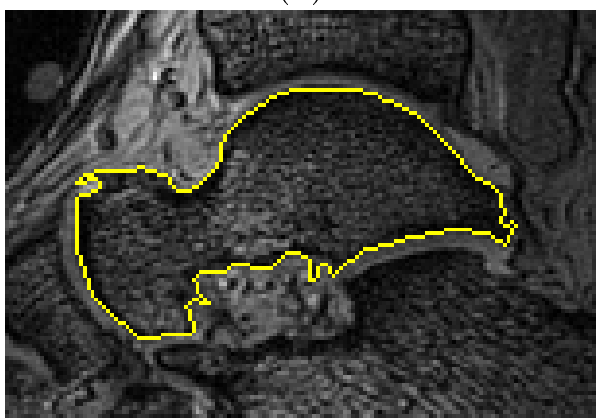

(d)

Figura 6.5: (a) Gabarito da segmentação do osso tálus em uma fatia de imagem RM do pé. (b) Exemplo de conjuntos de sementes obtidos pela erosão do gabarito de segmentação. (c) Resultado da segmentação por $f_{\max }^{b k g}$. (d) Um resultado melhor de segmentação é obtido mediante o uso da informação da orientação de borda do objeto por meio de $f_{i, \max }^{b k g}$.

O experimento para o conjunto de dados em 2D, constou de 80 imagens médicas obtidas por ressonância magnética (RM) e 80 imagens por tomografia computadorizada (TC), das quais 40 imagens RM foram usadas para segmentar o osso tálus, cuja curva de acurácia é apresentada na Figura 6.8(a), 40 imagens RM para segmentar o osso calcâneo, com curva de acurácia mostrada na Figura 6.8(b), 40 imagens TC pertencentes a 10 indivíduos para segmentar a espinha vertebral, com curva de acurácia dada na Figura 6.8(c), e 40 imagens TC de estudos torácicos de 10 indivíduos para segmentar o fígado, com curva de acurácia na Figura 6.8(d). Essas bases com gabaritos foram fornecidas por um especialista do departamento de radiologia da Universidade da Pensilvânia. Os marcadores automáticos para gerar as curvas de acurácia foram obtidos pelo procedimento de erosão do gabarito (Subseção 2.5.2).

No experimento em 3D, foi utilizado um conjunto de imagens RM-T1 do cérebro humano de 20 indivíduos normais, para segmentar o cerebelo, cuja curva de acurácia é apresentada na Figura 6.9.

Note que os resultados da avaliação experimental mostram um ganho considerável em termos 


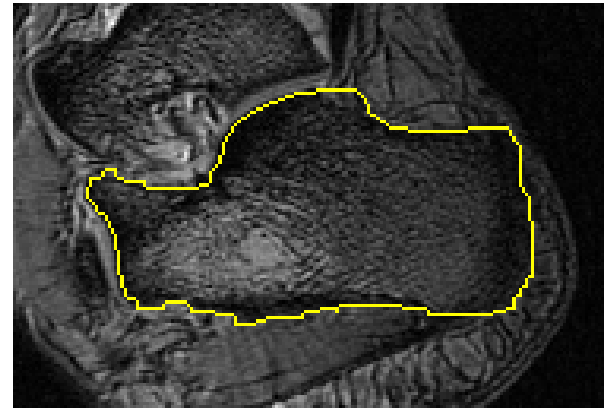

(a)

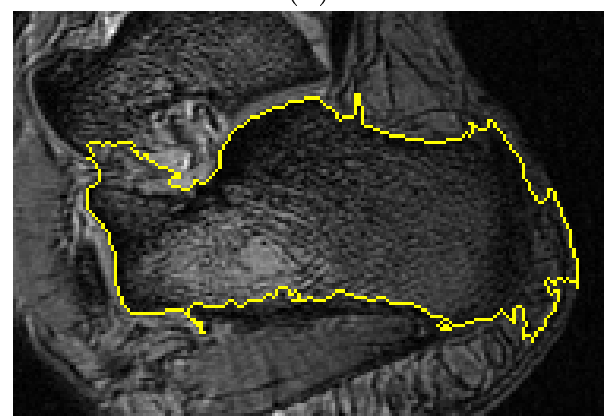

(c)

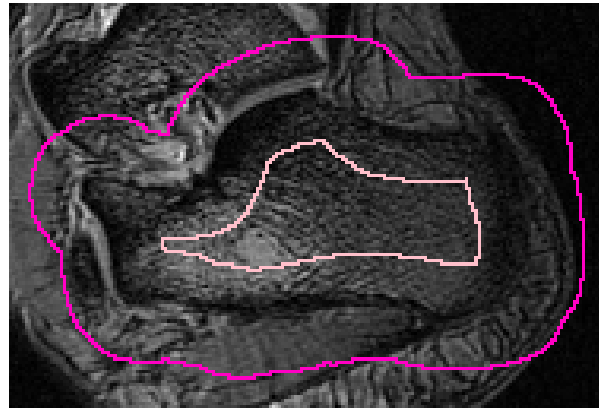

(b)

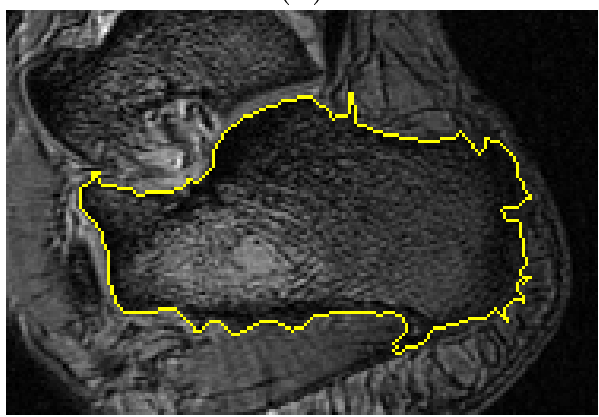

(d)

Figura 6.6: (a) Gabarito da segmentação do osso calcâneo em uma imagem RM do pé. (b) Exemplo de conjuntos semente obtidos pela erosão do gabarito. (c) Resultado da segmentação por f $f_{\max }^{b k g}$. (d) Um resultado melhor de segmentação é obtido explorando a orientação de borda usando $f_{i, \max }^{b k g}$.

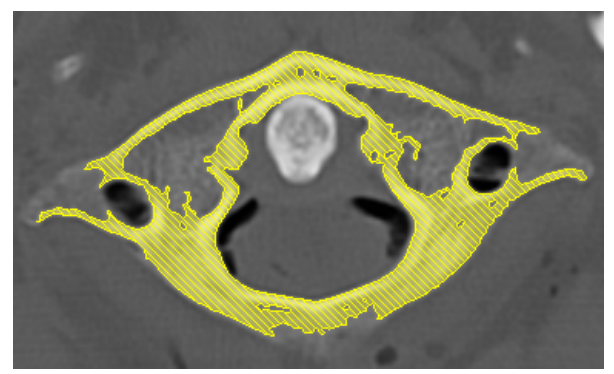

(a)

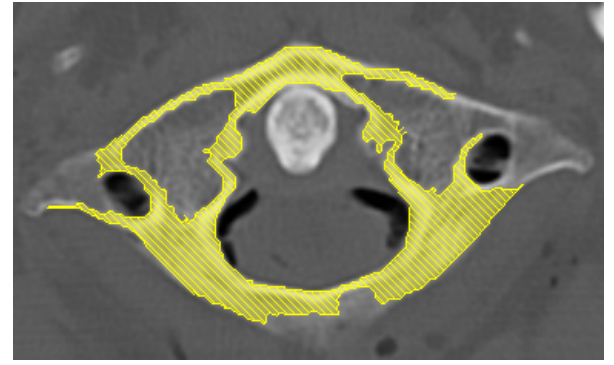

(c)

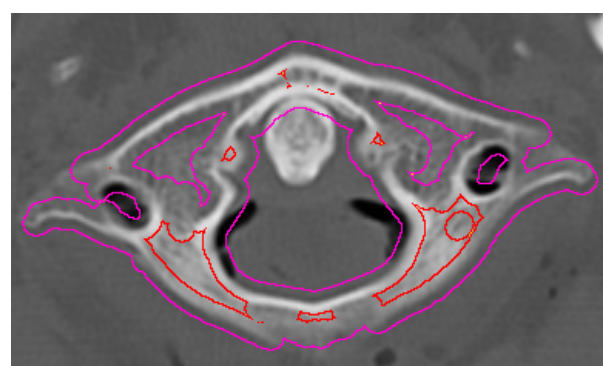

(b)

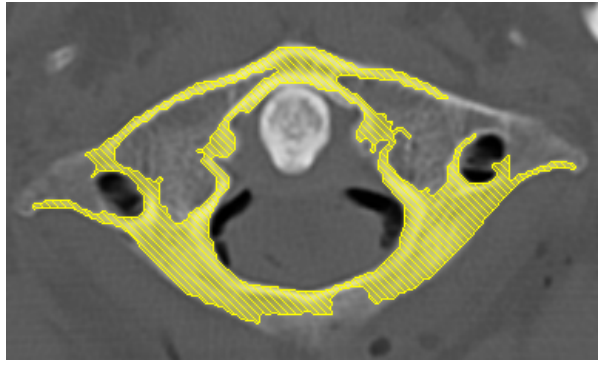

(d)

Figura 6.7: (a) Gabarito da segmentação da coluna vertebral em uma imagem TC. (b) Exemplo de conjuntos semente obtidos erodindo o gabarito da segmentação. (c) Resultado da segmentação por $f_{\max }^{b k g}$. (d) Um resultado melhor da segmentação é obtido explorando a orientação de borda usando $f_{o, \max }^{b k g}$.

de acurácia, quando exploramos a orientação de borda correta para o objeto em questão. 


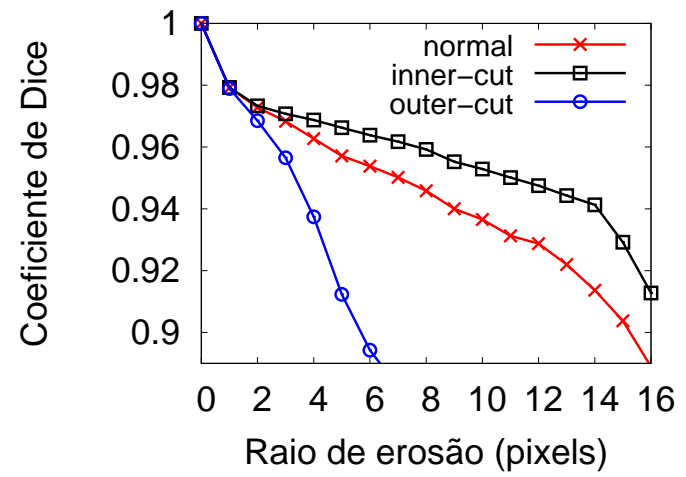

(a)

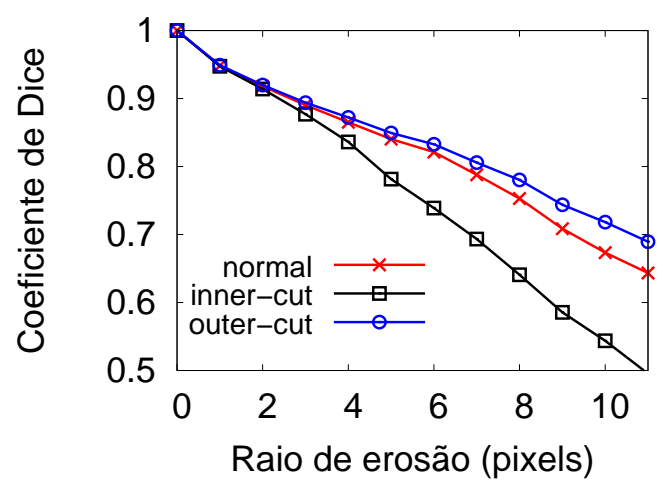

(c)

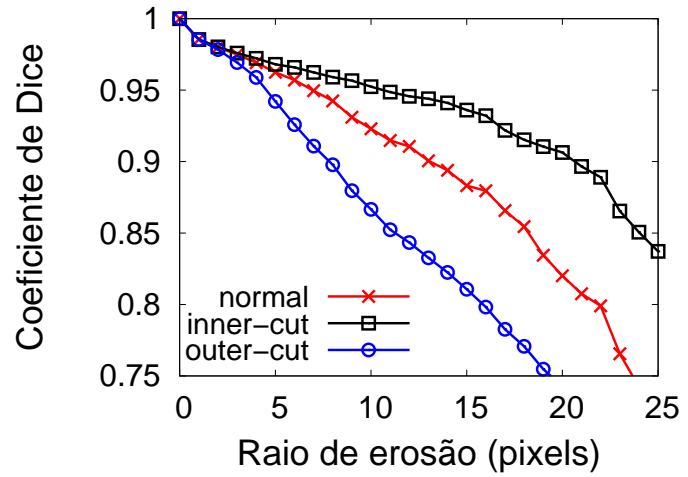

(b)

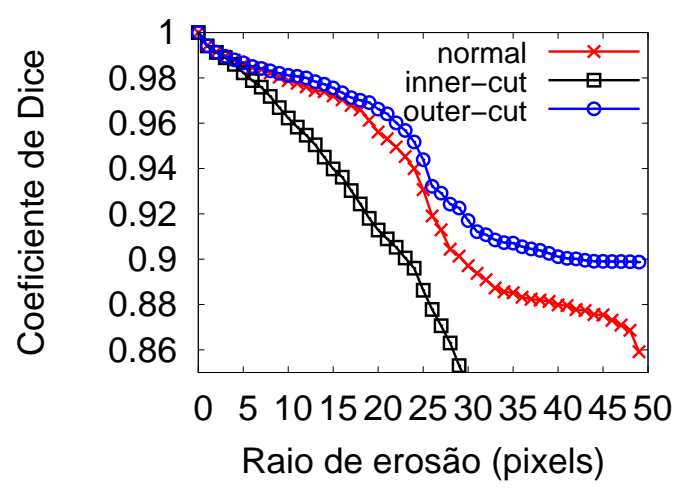

(d)

Figura 6.8: Curvas médias de acurácia para $f_{\max }^{b k g}$ (normal), $f_{i, \max }^{b k g}$ (inner-cut), $f_{o, \max }^{b k g}$ (outer-cut) para a segmentação do (a) tálus, (b) calcâneo, (c) coluna vertebral, e (d) fígado.

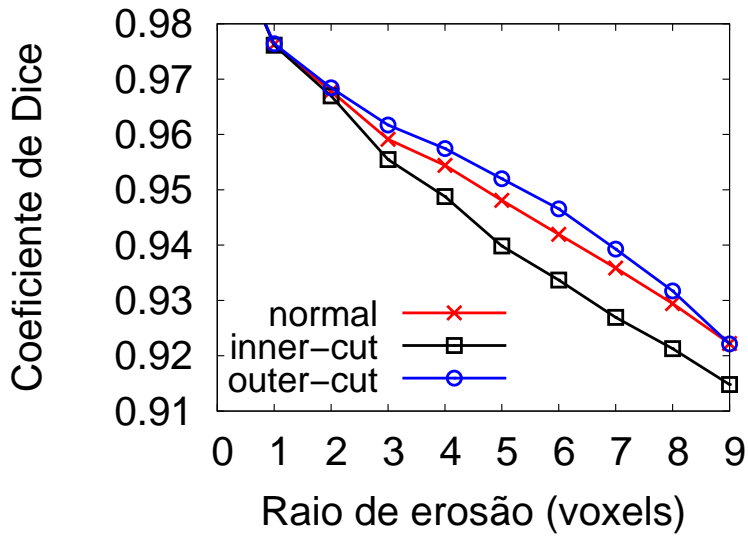

Figura 6.9: Curvas médias de acurácia para $f_{\max }^{b k g}$ (normal), $f_{i, \max }^{b k g}$ (inner-cut), $f_{o, \max }^{b k g}$ (outer-cut) para a segmentação $3 D$ do cerebelo.

\subsection{Segmentação pela OIFT com as funções de conexidade não su- aves $f_{i, \omega}$ e $f_{o, \omega}$}

Também modificamos a função $f_{\omega}$, obtendo as funções de conexidade não suaves $f_{i, \omega}$ e $f_{o, \omega}$, definidas por: 


$$
\begin{aligned}
f_{i, \omega}(\langle t\rangle) & = \begin{cases}-1 & \text { se } t \in \mathcal{S}_{o} \cup \mathcal{S}_{b} \\
+\infty & \text { caso contrário }\end{cases} \\
f_{i, \omega}\left(\pi_{s} \cdot\langle s, t\rangle\right) & = \begin{cases}\omega(t, s) & \text { se } R\left(\pi_{s}\right) \in \mathcal{S}_{o} \\
\omega(s, t) & \text { se } R\left(\pi_{s}\right) \in \mathcal{S}_{b}\end{cases}
\end{aligned}
$$

$$
\begin{aligned}
f_{o, \omega}(\langle t\rangle) & = \begin{cases}-1 & \text { se } t \in \mathcal{S}_{o} \cup \mathcal{S}_{b} \\
+\infty & \text { caso contrário }\end{cases} \\
f_{o, \omega}\left(\pi_{s} \cdot\langle s, t\rangle\right) & = \begin{cases}\omega(s, t) & \text { se } R\left(\pi_{s}\right) \in \mathcal{S}_{o} \\
\omega(t, s) & \text { se } R\left(\pi_{s}\right) \in \mathcal{S}_{b}\end{cases}
\end{aligned}
$$

As funções $f_{i, \omega}$ e $f_{o, \omega}$ permitem cortes ótimos que maximizam as Equações 6.1 e 6.2, respectivamente (Teorema 3), lidando melhor com os casos de empate entre bordas com mesma orientação.

Teorema 3 (Otimalidade de cortes internos/externos) Dado dois conjuntos $\mathcal{S}_{o}$ e $\mathcal{S}_{b}$ de sementes, qualquer floresta de espalhamento calculada pelo algoritmo da IFT (Algoritmo 1) para a função $f_{o, \omega}$ define um corte ótimo que maximiza $E_{o}(\hat{L})$ entre todos os resultados possiveis de segmentação satisfazendo as restrições fortes. Qualquer floresta de espalhamento calculada pelo Algoritmo 1 para a função $f_{i, \omega}$ define um corte ótimo que maximiza $E_{i}(\hat{L})$ entre todos os resultados possiveis de segmentação satisfazendo as restrições fortes.

A prova do Teorema 3 foi apresentada em [Mansilla e Miranda (2013a)], a qual é similar à prova do Teorema 2, por isso não apresentaremos sua prova neste trabalho.

A diferença em relação as funções apresentadas anteriormente (Seção 6.2) é que $f_{o, \max }^{b k g}\left(f_{i, \max }^{b k g}\right)$ apresenta uma única solução ótima de segmentação [Miranda e Mansilla (2014)], ao passo que $f_{o, \omega}$ $\left(f_{i, \omega}\right)$ pode oferecer múltiplas soluções ótimas para o mesmo problema de maximização de energia, dependendo da política de desempate usada na fila de prioridade.

A Figura 6.10 mostra como $f_{o, \omega}$ lida melhor com empates entre múltiplas bordas de mesma energia em comparação com $f_{o, \max }^{b k g}$, permitindo uma divisão mais equilibrada nestas regiões ambíguas.

\subsubsection{Resultados experimentais}

Nos experimentos realizados consideramos $f_{o, \omega}$ e $f_{i, \omega}$ com uma política de desempate FIFO, a qual permite uma maior acurácia em comparação com $f_{o, \max }^{b k g} f_{i, \max }^{b k g}$. Os resultados foram avaliados em 20 imagens volumétricas de RM reais do pé para segmentar os ossos tálus e calcâneo em 3D. As curvas de acurácia foram calculadas para os métodos definidos na Tabela 6.1, usando o procedimento de sementes automáticos por erosão do gabarito descrito na Subseção 2.5.2.

Os pesos das arestas foram calculados como uma combinação de uma medida regular de dissimilaridade não direcionada (por exemplo, as curvas de acurácia na Figura 6.11 consideram $\psi(p, q)=\mathcal{G}(p)+\mathcal{G}(q)$, onde $\mathcal{G}(p)$ é a magnitude do gradiente de Sobel no pixel $p$, e na Figura 6.12 é usado o valor absoluto da diferença de intensidades, ou seja $\psi(p, q)=|I(p)-I(q)|)$ multiplicada por um fator de orientação (Equação 6.3). 


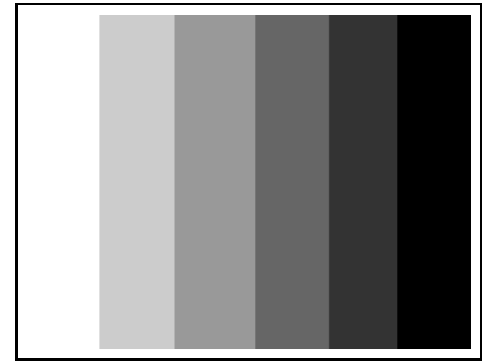

(a)

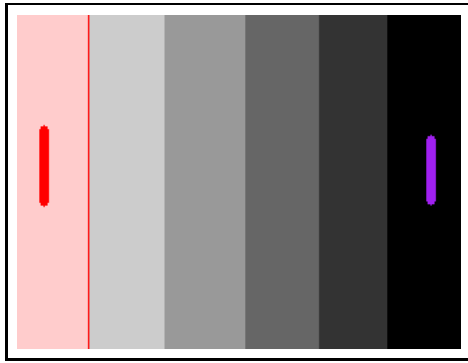

(b)

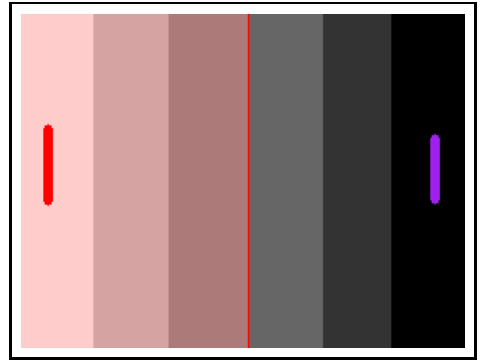

(c)

Figura 6.10: (a) Imagem de entrada com transições igualmente ponderadas, tendo a mesma orientação. (b) Resultado da OIFT usando $f_{o, \max }^{b k g}$ como proposto em [Miranda e Mansilla (2014)], atribuindo regiões ambiguas para o fundo. (c) OIFT usando $f_{o, \omega}$ com uma politica de desempate FIFO fornece partições mais igualmente balanceadas.

\begin{tabular}{|c|c|c|}
\hline Método & Descrição & Tipo de Grafo \\
\hline$I R F C$ & $\begin{array}{l}\text { Iterative Relative Fuzzy Connectedness [Ciesielski et al. (2007)] } \\
\text { (IFT com } f_{\max }^{\text {bkg }} \text { ) }\end{array}$ & grafo não dirigido \\
\hline$I F T_{F I F O}^{\max }$ & $\begin{array}{l}\text { IFT tradicional com } f_{\max } \text { e política de desempate FIFO como descrito } \\
\text { em [Falcão et al. (2004)] }\end{array}$ & grafo não dirigido \\
\hline$P W_{q=2}$ & Algoritmo power watershed [Couprie et al. (2010)] & grafo não dirigido \\
\hline OIFT $T_{\text {inner }}^{\max }$ & OIFT usando $f_{i, \max }^{b k g}$ como proposto em [Miranda e Mansilla (2014)] & grafo direcionado \\
\hline OIFT $T_{\text {outer }}^{\max }$ & OIFT usando $f_{o, \max }^{b k g}$ como proposto em [Miranda e Mansilla (2014)] & grafo direcionado \\
\hline OIFT $T_{\text {inner }}^{\omega}$ & OIFT usando $f_{i, \omega}$ com uma política de desempate FIFO & lirecionado \\
\hline OIFT $T_{\text {outer }}^{\omega}$ & OIFT usando $f_{o, \omega}$ com uma política de desempate FIFO & grafo direcionado \\
\hline
\end{tabular}

Tabela 6.1: Descrição dos métodos usados nos experimentos da segmentação pela OIFT com $f_{i, \omega}$ e $f_{o, \omega}$.

Os experimentos da Figura 6.12 retratam uma configuração dos pesos do grafo em que os empates são mais frequentes. A partir das Figuras 6.11 e 6.12 podemos observar o ganho de acurácia de $f_{i, \omega}$ sobre $f_{i, \max }^{b k g}$, e a sua maior robustez em relação aos pesos adotados para as arestas do grafo.

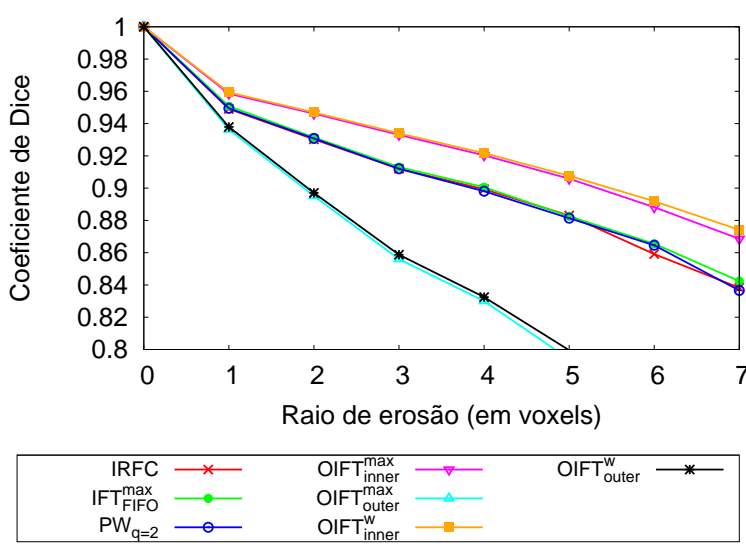

(a)

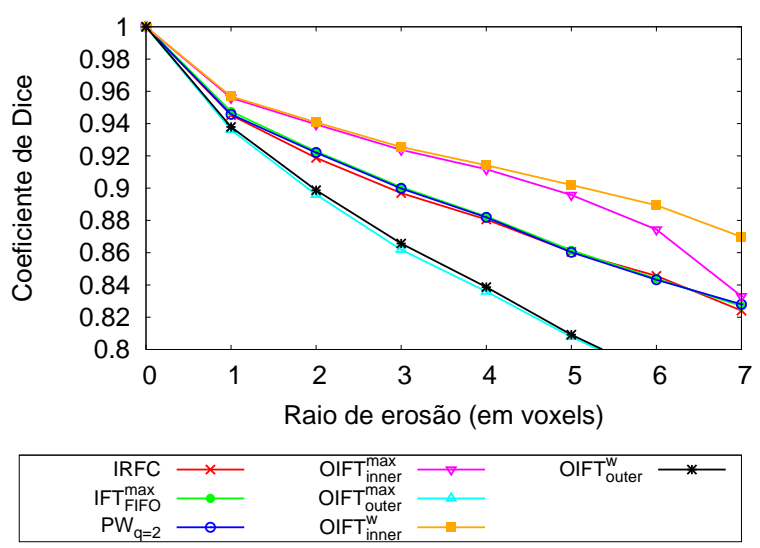

(b)

Figura 6.11: Curvas médias de acurácia (Dice) usando o gradiente Sobel para a segmentação 3D dos ossos: (a) tálus, e (b) calcâneo.

Também apresentamos uma aplicação da OIFT para a segmentação interativa de imagens coloridas, onde a função $f_{o, \omega}$ pode ser usada para melhorar a segmentação, utilizando a orientação de um mapa de pertinência calculado pela técnica proposta em [Miranda et al. (2010)]. Para o expe- 


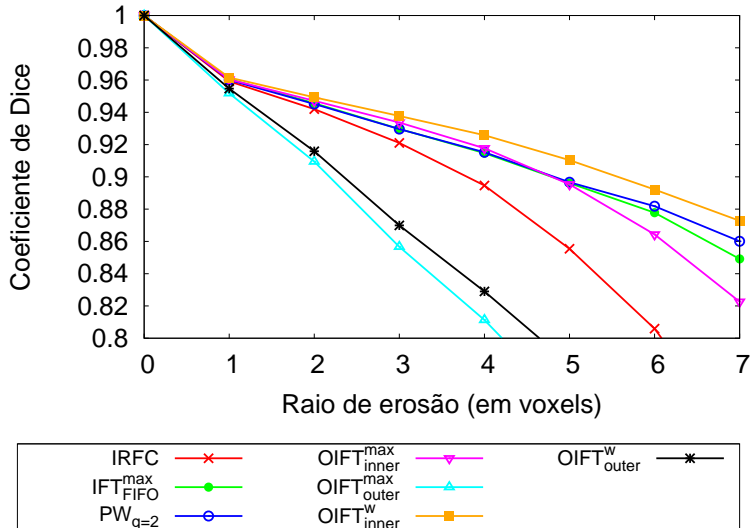

(a)

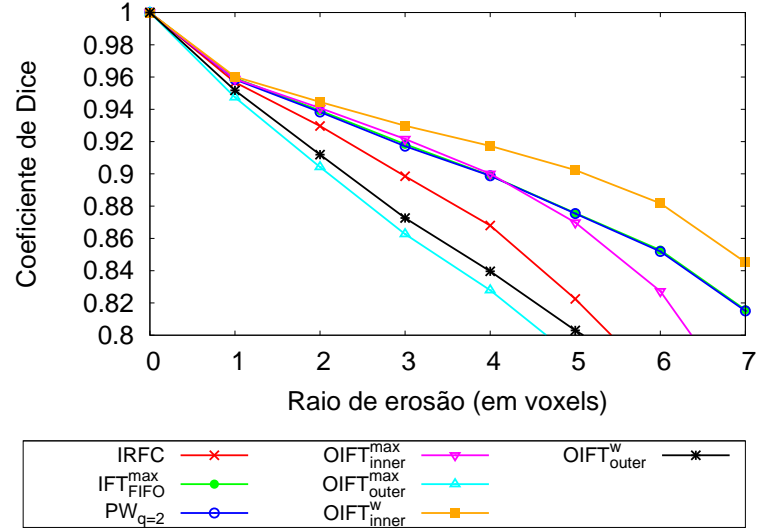

(b)

Figura 6.12: Curvas médias de acurácia (Dice) usando o valor absoluto da diferença de intensidades para a segmentação $3 D$ dos ossos: (a) tálus, e (b) calcâneo.

rimento fizemos o uso de 50 imagens naturais com gabaritos conhecidos obtidas de [Rother et al. ]. As curvas de acurácia são mostradas na Figura 6.13, onde $f_{o, \omega}$ apresenta um melhor resultado. No entanto, cabe mencionar que $f_{o, \max }^{b k g}$ (não mostrado) apresentou um resultado muito similar a $f_{o, \omega}$ nessa aplicação.

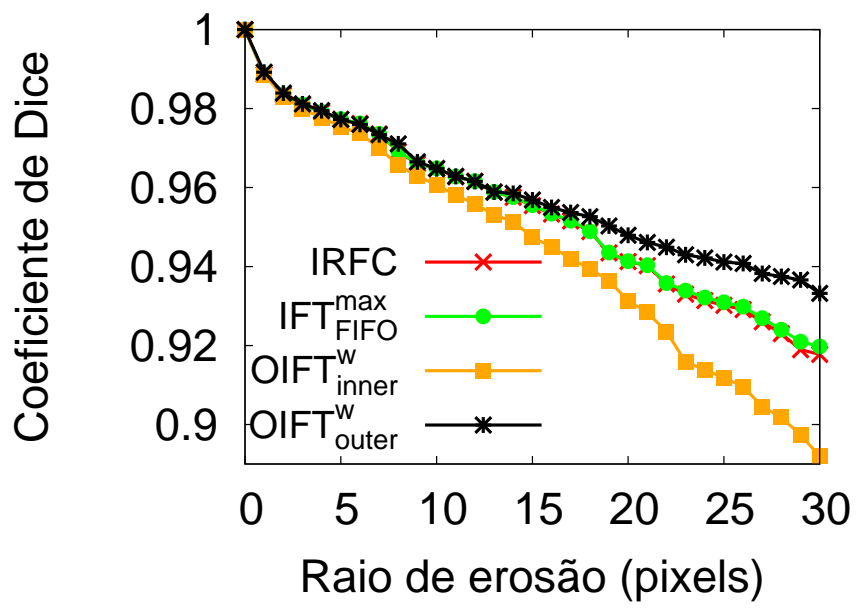

Figura 6.13: Curvas médias de acurácia para diferentes métodos usando imagens coloridas. 


\section{Capítulo 7}

\section{Transformada imagem-floresta com convexidade geodésica em estrela}

Estruturas anatômicas e tecidos são, geralmente, difíceis de segmentar em imagens médicas devido ao baixo contraste das suas bordas podendo gerar resultados não desejados (falsas bordas), com formas não regulares (Figura 7.1), motivo pelo qual o uso de restrições de forma pode ajudar a reduzir esse tipo de problema para objetos que tem uma forma mais regular.

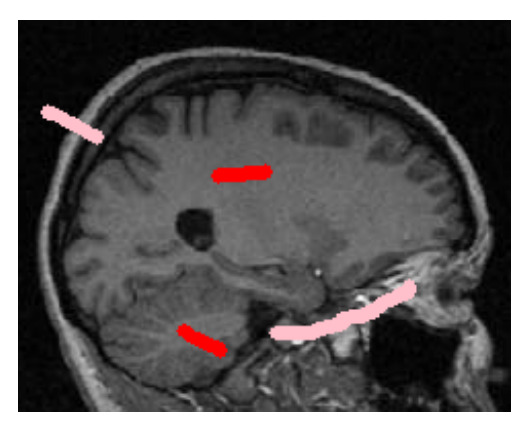

(a)

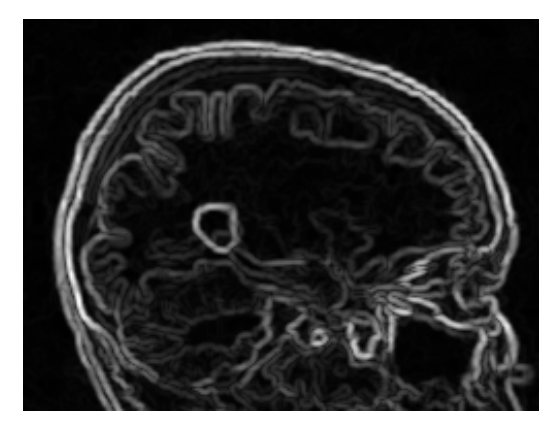

(b)

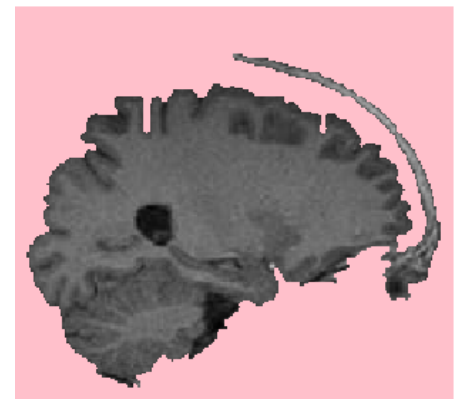

(c)

Figura 7.1: Em (a) consideramos uma imagem RM do cérebro (objeto de forma regular). Em (b) é mostrado o gradiente da imagem. Em (c) temos o resultado da segmentação do cérebro pela IFT-SC com $f_{\max }$ que apresenta uma forma não regular.

Recentemente, Gulshan et al. [Gulshan et al. (2010)] incorporaram uma restrição de convexidade geodésica em estrela (GSC, Geodesic Star Convexity) para segmentação interativa sob um enfoque de corte em grafo por min-cut/max-flow. Neste capítulo nós apresentamos uma extensão do algoritmo da IFT-SC [Miranda e Falcão (2009)] considerando esse mesmo tipo de restrição.

\subsection{Convexidade em estrela}

Um objeto atende a convexidade em estrela em relação a um ponto central $c$, se para todo ponto $p$ do objeto, todos os pontos do segmento de reta ligando $c$ a $p$ também fazem parte do objeto. Sendo assim, qualquer forma convexa ${ }^{1}$, contendo o ponto $c$, é também uma forma que atende a

\footnotetext{
${ }^{1}$ Uma forma é convexa se para quaisquer pares de pontos no objeto, o segmento de reta interligando eles está também contido no objeto.
} 
restrição de convexidade em forma de estrela, porém nem toda forma em estrela é convexa, de modo que a restrição de convexidade em estrela é mais flexível (Figura 7.2).

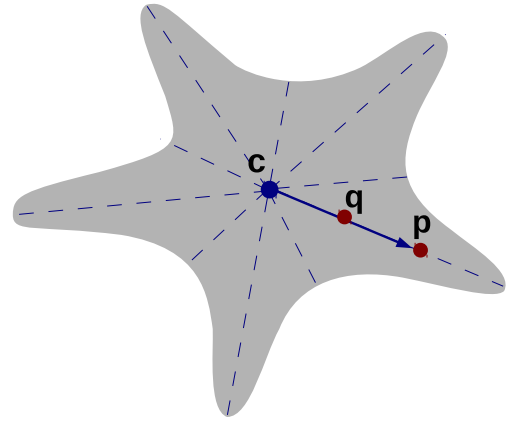

(a)

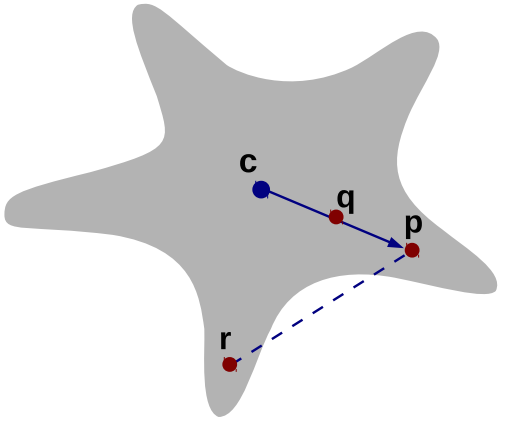

(b)

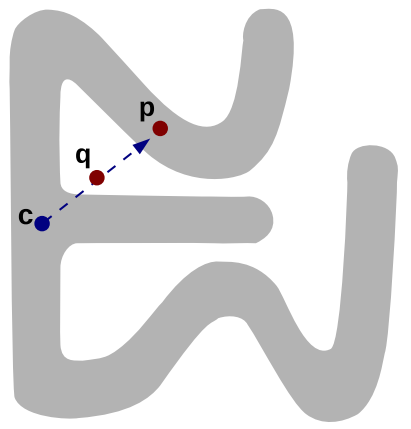

(c)

Figura 7.2: Convexidade em estrela: Em (a) é apresentado um objeto convexo em estrela, o qual não é convexo como mostrado em (b). Um objeto que viola a restrição de convexidade em estrela é mostrado em (c), onde p é um ponto do objeto dado, e c é o centro de estrela considerado.

A convexidade em estrela pode ser interpretada como uma restrição de visibilidade, isto é, um objeto é convexo em estrela em relação ao ponto central $c$, se para todo ponto $p$ no objeto, $p$ é visível a $c$ via o segmento de reta interligando eles, o qual também deve fazer parte do objeto (o fundo é considerado como um obstáculo que obstrui a "luz" emitida por $p$ até o observador $c$ ). Uma possível extensão para vários centros de estrela (Figura 7.3(a)), considera que os pontos do objeto devem ser visíveis em relação a pelo menos um dos centros. Porém esta definição é computacionalmente intratável, estando relacionada a um problema NP-completo [Gulshan et al. (2010)]. Assim, uma alternativa de solução computacionalmente factível é considerar o argumento da distância mais curta (Figura 7.3(b)). Essa alternativa pode ser definida em uma versão discreta no domínio da imagem $\hat{I}$ (Figura 7.3(c)), onde os segmentos de reta correspondem a caminhos no grafo derivado da imagem.

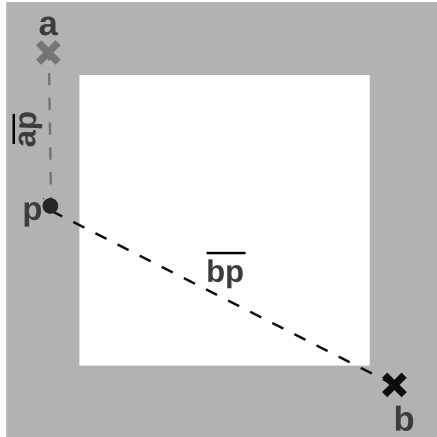

(a)

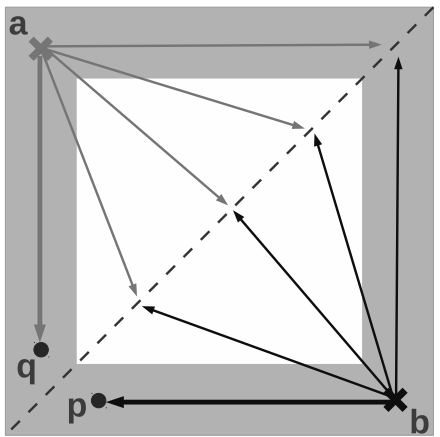

(b)

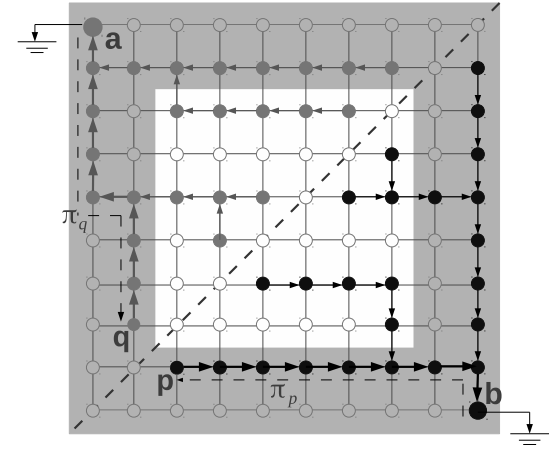

(c)

Figura 7.3: Considere um objeto $\mathcal{O}$ (área sombreada), com o conjunto de centros de estrela $C=\{a, b\}$. Em (a) temos o argumento de visibilidade: Qualquer ponto $p \in \mathcal{O}$ deve ser visivel ao menos por um dos centros de estrela (por exemplo, $p$ é visível através de $\overline{a p}$, embora este não seja visivel através de $\overline{b p}$ ). Em (b) temos o argumento da distancia mais curta: Cada ponto é analisado exclusivamente em relação a seu centro mais próximo (por exemplo, q está mais próximo de a, e p mais próximo de b). Em (c) temos uma versão discreta pelo caminho mais curto no grafo de imagem. 


\subsection{Segmentação com restrições de convexidade geodésica em es- trela}

Nós consideramos os centros de estrela como as sementes internas selecionadas pelo usuário, e os segmentos de reta como os caminhos ligando cada ponto do objeto ao centro de estrela mais próximo formando uma floresta de caminhos de custo geodésico mínimo da IFT.

Assim, nós definimos os pesos das arestas $\delta(s, t)$ na regra de extensão de caminho para $f_{\text {sum }}$ (Equação 3.1) como:

$$
\delta(s, t)=[\omega(s, t)+1]^{\beta}-1+\|t-s\|
$$

onde $\|t-s\|$ é a distância Euclideana entre os pixels $s$ e $t$, e $\beta$ controla a topologia da floresta. Para valores muito pequenos de $\beta(\beta \approx 0.0), \delta(s, t)$ aproxima-se de $\|t-s\|$, e a topologia da floresta torna-se similar à floresta de caminhos ótimos Euclideanos (Figura 7.4(a)). Para valores maiores, $[\omega(s, t)+1]^{\beta}$ domina a expressão, e quanto maior for a dissimilaridade baseada em intensidade, maior será a sua influência sobre os resultados (Figuras 7.4(b), 7.4(c) e 7.4(d)). Ou seja, dependendo de como são configurados os pesos das arestas tem-se caminhos que seguem raios euclidianos ou que se adaptam as formas presentes na imagem fazendo curvas, de acordo com o parâmetro de controle $\beta$.

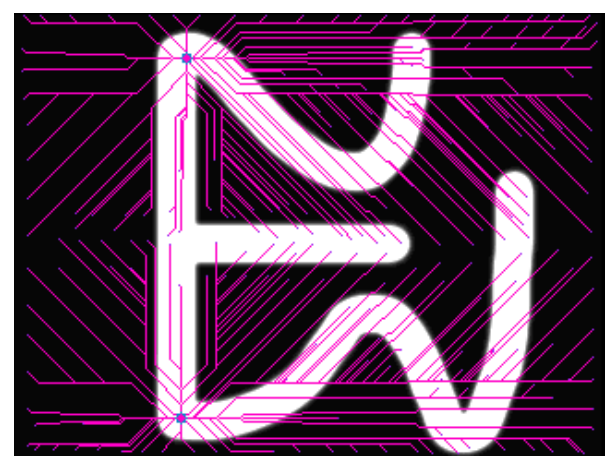

(a)

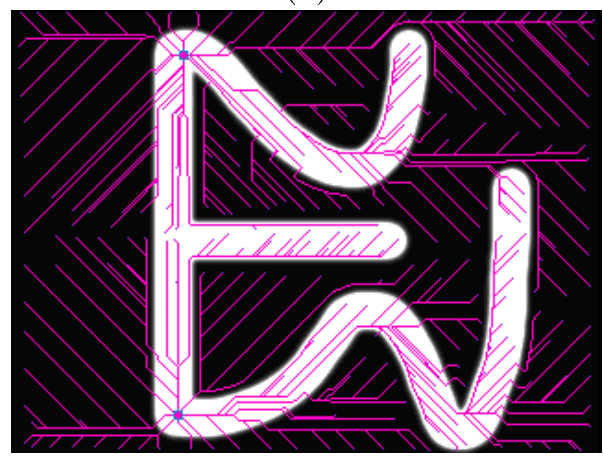

(c)

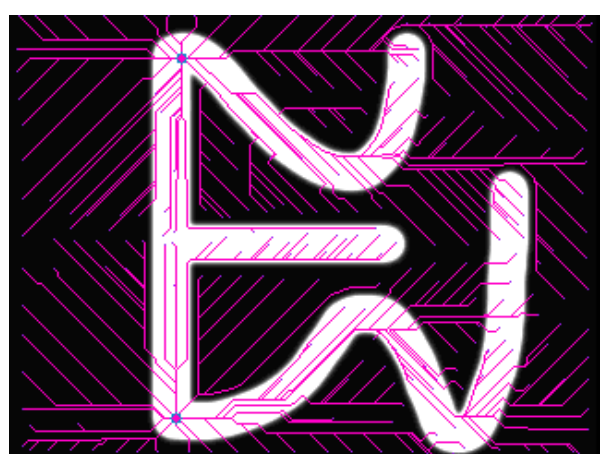

(b)

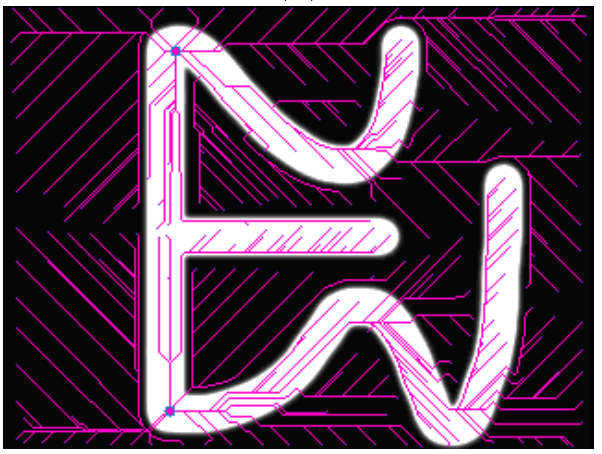

(d)

Figura 7.4: Efeito do parâmetro de potência $\beta$ sobre a topologia da floresta: A floresta de caminhos ótimos de $f_{\text {sum }}$ para (a) $\beta=0.0$, (b) $\beta=0.3$, (c) $\beta=0.4$, e (d) $\beta=0.5$.

A Figura 7.5 mostra como o parâmetro $\beta$ afeta a floresta para $f_{\text {sum }}$, e sua correspondente segmentação com restrições de forma (Algoritmo 2). Claramente, valores mais baixos de $\beta$ impõem mais regularização para a borda do objeto, enquanto valores mais elevados permitem um melhor 
ajuste para protuberâncias e saliências na borda.

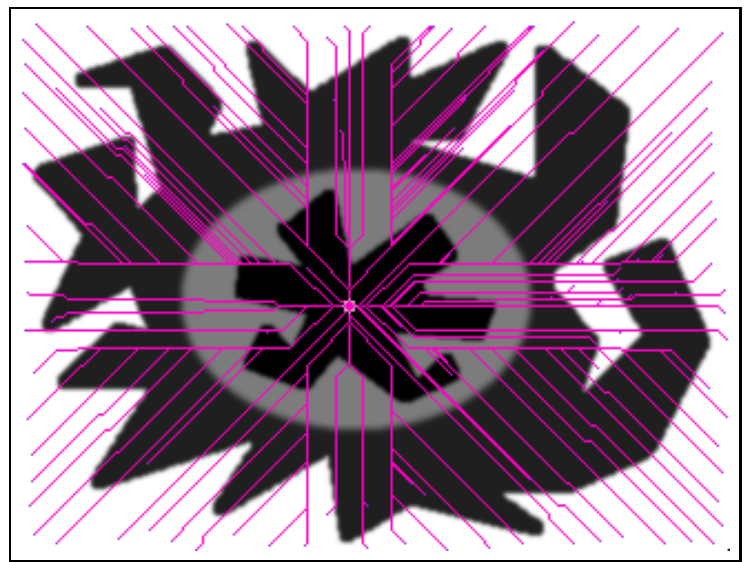

(a)

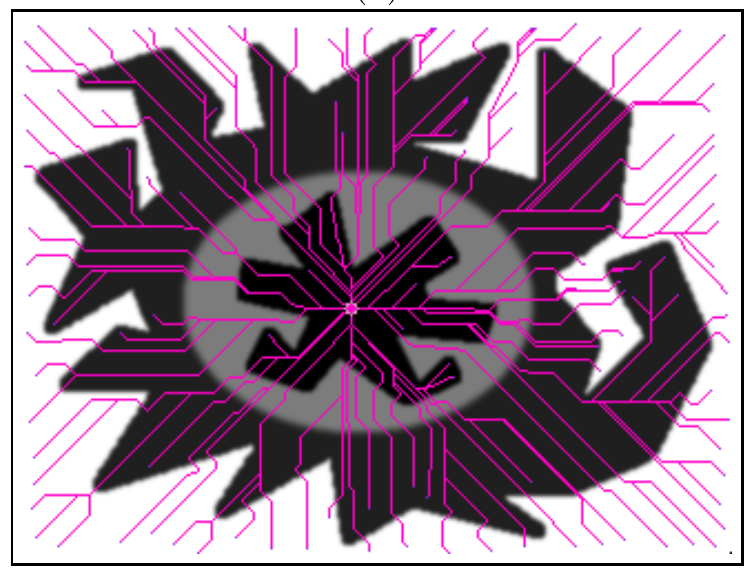

(c)

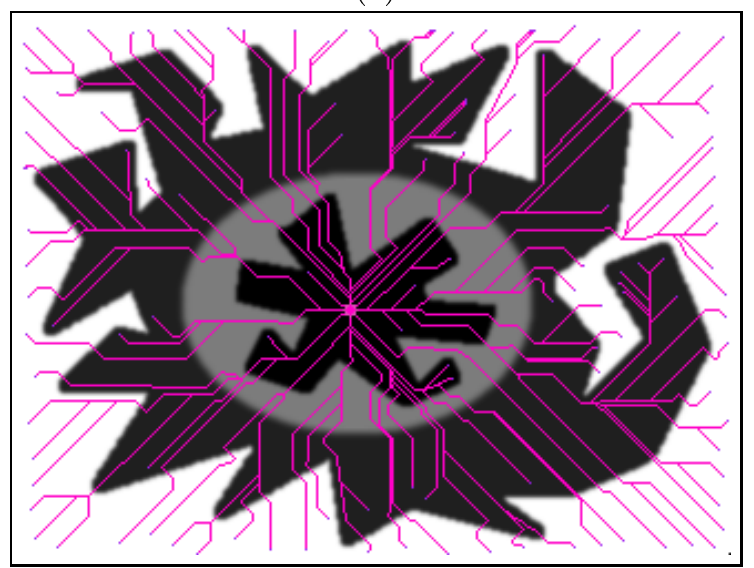

(e)

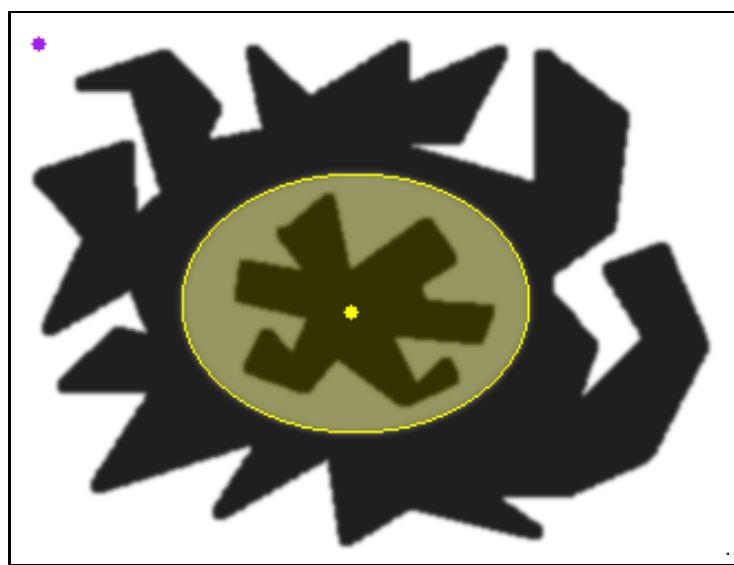

(b)

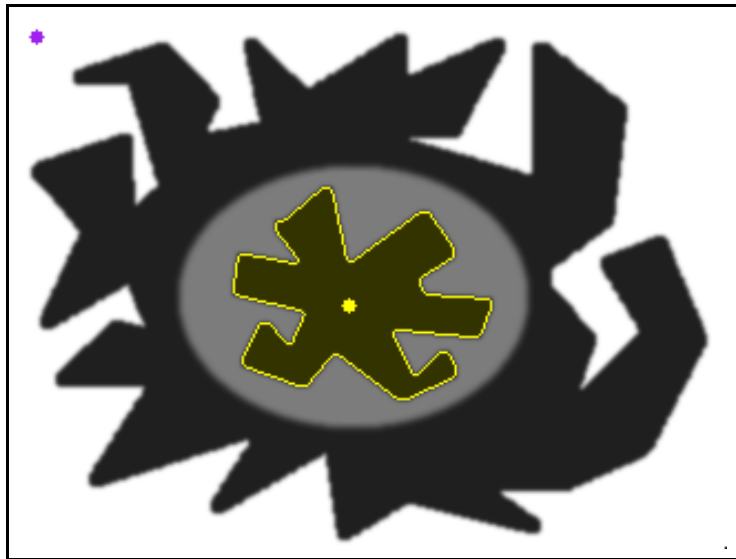

(d)

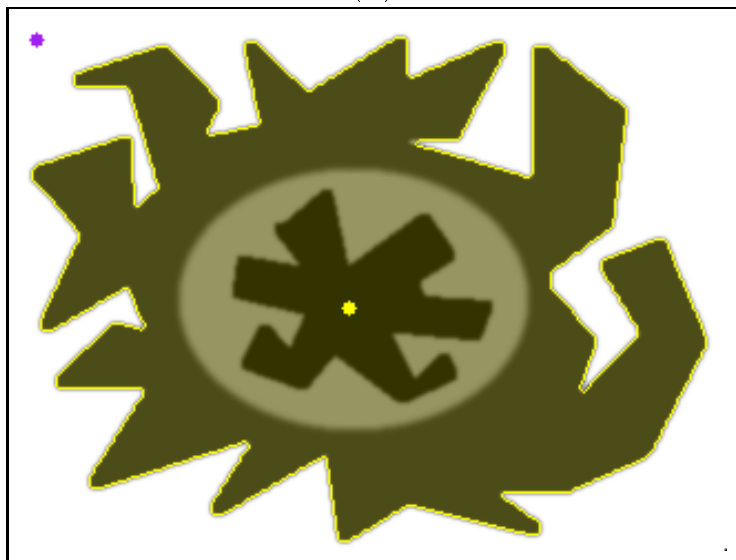

(f)

Figura 7.5: Efeitos do parâmetro de potência $\beta$ sobre a topologia da floresta: Em (a) é mostrada a floresta de caminhos ótimos de $f_{\text {sum }}$ para $\beta=0$. Em (b) é mostrada a segmentação resultante pelo algoritmo GSCIFT (Algoritmo 2). Resultados similares são mostrados em (c) e (d) para $\beta=0.3$, e em (e) e (f) para $\beta=0.5$.

\subsection{Algoritmo GSC-IFT}

O Algoritmo 2 obtém a segmentação que maximiza a medida de corte em grafo $E$ (Equação 4.3) entre todos os possíveis resultados satisfazendo as restrições de forma pela convexidade geodésica 
em estrela (GSC), cuja prova de otimalidade é apresentada no Apêndice D.

\section{Algoritmo 2 - Algoritmo GSC-IFT}

Entrada: $\quad$ Grafo $G=(\mathcal{I}, \xi)$, e conjunto $\mathcal{S}_{o}$ e $\mathcal{S}_{b}$ de sementes.

SAídA: $\quad$ Mapa $V$ de custos mínimos, e mapa $L$ de rótulos.

Auxiliares: Floresta $P_{\text {sum }}$ de caminhos ótimos, fila $Q$ de prioridade, variável tmp, e um array estado.

1. Calcule $P_{\text {sum }} \leftarrow I F T-S C\left(G, \mathcal{S}_{o}, \emptyset, f_{\text {sum }}\right)$.

2. Para cada $t \in \mathcal{S}_{o}$, faça $L(t) \leftarrow 1$.

3. Para cada $t \in \mathcal{S}_{b}$, faça $L(t) \leftarrow 0$.

4. Para cada $t \in \mathcal{I}$, faça

5. $\quad V(t) \leftarrow f_{\omega}(\langle t\rangle)$, e estado $(t) \leftarrow 0$.

6. $\quad$ Se $V(t) \neq+\infty$, então insira $t$ em $Q$.

7. Enquanto $Q \neq \emptyset$, faça

8. $\quad$ Remova s de $Q$ cujo valor $V(s)$ é mínimo.

9. $\quad$ Se $L(s)=1$, então

10. $\quad \mathrm{L}$ Conquistar_Caminho( $s, G, P_{\text {sum }}, V, Q, L$, estado $)$.

11. Senão Se $L(s)=0$, então

12. $L \quad \mathrm{~L} \quad$ Podar_Árvore $\left(s, G, P_{\text {sum }}, V, Q, L\right.$, estado $)$.

\section{Algoritmo 3 - Algoritmo Conquistar_Caminho}

Entrada: $\quad$ Pixel $s$ da imagem $(s \in \mathcal{I})$, grafo $G=(\mathcal{I}, \xi)$ da imagem, floresta $P_{\text {sum }}$ de caminhos ótimos, mapa $V$ de custos mínimos, fila $Q$ de prioridade, mapa $L$ de rótulos, e um array estado.

Auxiliares: Variáveis tmp, $x$ e $y$.

1. $x \leftarrow s$.

2. Faça

3. $\quad$ Se $x \in Q$, então remova $x$ de $Q$

4. $\quad \operatorname{estado}(x) \leftarrow 1$, e $L(x) \leftarrow 1$.

5. $\quad$ Para cada $y \in \mathcal{A}(x)$, tal que estado $(y) \neq 1$, faça

6. $\quad$ Calcule tmp $\leftarrow \omega(x, y)$.

7. $\quad$ Se $t m p<V(y)$ e $y \neq \operatorname{Psum}(x)$, então

8. $\quad$ Se $y \in Q$, então remova y de $Q$.

9. $\quad[\quad$ Faça $V(y) \leftarrow t m p, L(y) \leftarrow 1$, e insira y em $Q$.

10. $\quad x \leftarrow \operatorname{Psum}(x)$.

11. $[$ Enquanto $(x \neq$ nil e estado $(x) \neq 1)$

\section{Algoritmo 4 - Algoritmo Podar_Árvore}

Entrada: Os mesmos parâmetros como no Algoritmo 3.

Auxiliares: Fila FIFO $Q_{F I F O}$, variáveis $t m p, x$, e $y$.

1. $x \leftarrow s$.

2. Se $x \in Q$, então remova $x$ de $Q$

3. Faça estado $(x) \leftarrow 1, L(x) \leftarrow 0$, e insira $x$ em $Q_{F I F O}$.

4. Enquanto $Q_{F I F O} \neq \emptyset$

5. Remova $x$ de $Q_{F I F O}$.

6. $\quad$ Para cada $y \in \mathcal{A}(x)$, tal que estado $(y) \neq 1$, Faça

7.

Se $\operatorname{Psum}(y)=x$, então 


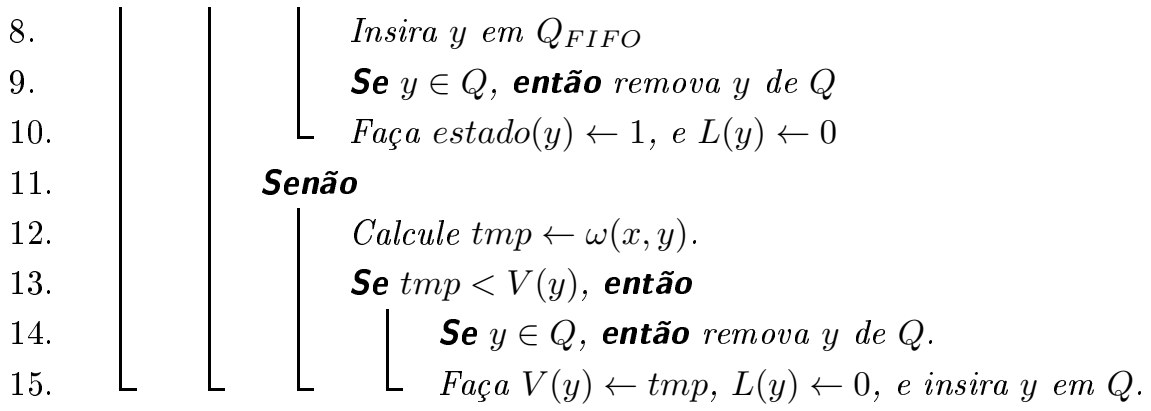

Na Linha 1, a floresta ótima $P_{\text {sum }}$ para a função $f_{\text {sum }}$ (Equação 3.1) é calculada invocando o algoritmo regular da IFT-SC (Algoritmo 1), usando somente o conjunto $\mathcal{S}_{o}$ (conjunto de centros de estrela). As Linhas 2 a 8 são similares às linhas originais da IFT-SC com a função não suave $f_{\omega}$ (Equação 4.2). As diferenças surgem a partir desse ponto (Linhas 9 a 12).

Quando um pixel $s$ sai da fila $Q$ de prioridade com rótulo $L(s)=1$, temos que nenhum de seus predecessores $x$ na floresta $P_{\text {sum }}$ poderá ter rótulo $L(x)=0$, pois isso implicaria em uma violação da visibilidade de $s$ em relação a sua semente mais próxima (Figura 7.6(b)). Portanto, devemos executar o procedimento "Conquistar_Caminho" (Algoritmo 3). Esse procedimento é similar a executar as Linhas 9 a 14 do algoritmo da IFT-SC (Algoritmo 1) não somente em relação ao pixel $s$, mas também para todos seus predecessores em $P_{\text {sum }}$, e atribuindo seus rótulos para 1 (Figura 7.6(c)).

No caso no qual o pixel $s$ sai da fila de prioridade com rótulo $L(s)=0$, temos que nenhum de seus descendentes na floresta $P_{\text {sum }}$ (subárvore de $P_{\text {sum }}$ enraizada em $s$ ) poderá ter rótulo 1 , pois isso resultaria em uma violação da visibilidade desses descendentes em relação a sua semente mais próxima em $P_{\text {sum }}$ (Figura 7.6(d)). Portanto, nós devemos executar o procedimento "Podar_Árvore" (Algoritmo 4). Esse procedimento também é similar a executar as Linhas 9 a 14 do algoritmo da IFT-SC (Algoritmo 1) para todos os descendentes de $s$, e atribuindo seus rótulos para 0 (Figura 7.6(e)).

A Figura 7.6 ilustra um exemplo de segmentação fazendo uso do algoritmo GSC-IFT (Algoritmo 2).

\subsection{Resultados experimentais}

Nos experimentos comparamos diferentes algoritmos para segmentar um conjunto de imagens médicas, os quais são: IRFC [Ciesielski et al. (2012b)], power watershed $\left(P W_{q=2}\right)$ [Couprie et al. (2010)], e o algoritmo proposto da IFT com Convexidade Geodésica em Estrela (GSC-IFT). Estes métodos foram considerados pelo fato de serem abordagens competitivas baseadas na mesma energia, permitindo uma clara visão da vantagem do uso da restrição GSC na otimização desta energia. Nos experimentos, fez-se uso de 40 fatias de imagens de RM reais do pé para todos os métodos mencionados para segmentar os ossos calcâneo e tálus, 40 fatias de imagens de TC de estudos torácicos de 10 indivíduos para segmentar o fígado, e 40 fatias de imagens de RM de mama. As curvas de acurácia são (obtidas pelo procedimento descrito na Subseção 2.5.2) mostradas na Figura 7.7, as quais apresentam um ganho significativo para o algoritmo proposto (GSC-IFT), exceto para o conjunto de dados do tálus onde os ganhos foram apenas ligeiramente melhores. Além disso, para o mesmo conjunto de imagens apresentamos uma distribuição do ranqueamento dos métodos de acordo com a frequência de classificação por comparação das médias do coeficiente de Dice (Figura 7.8). 


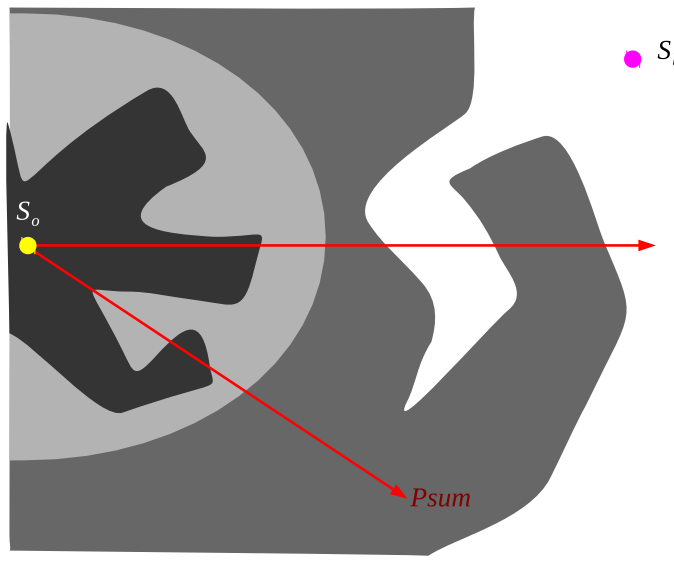

(a)

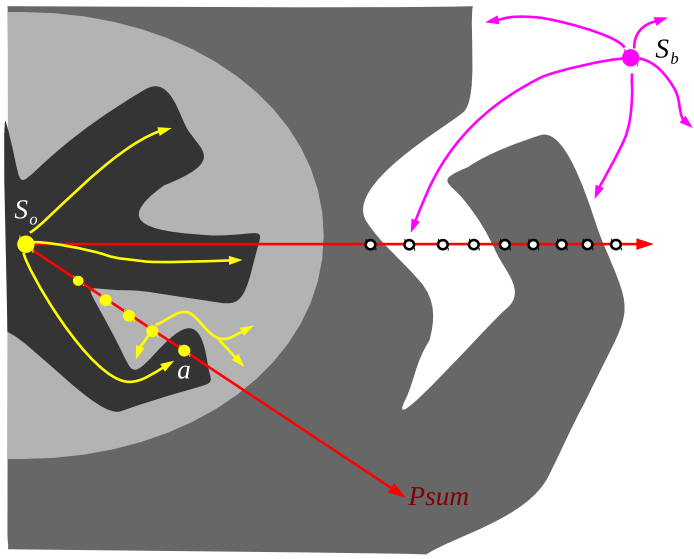

(c)

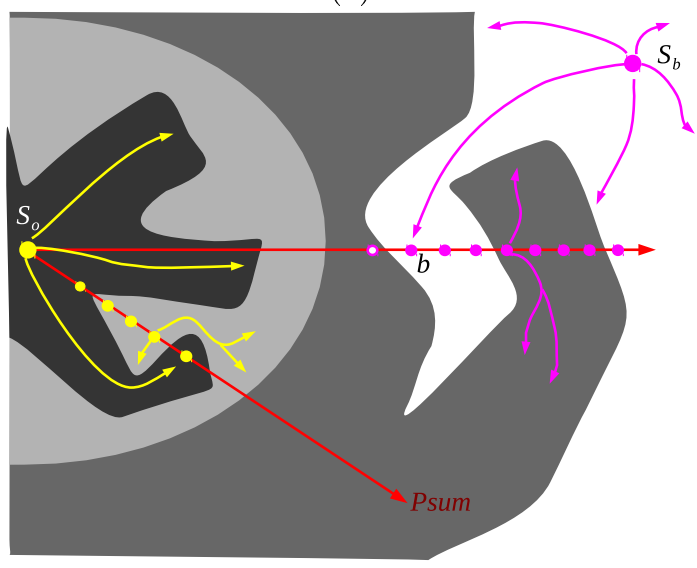

(e)

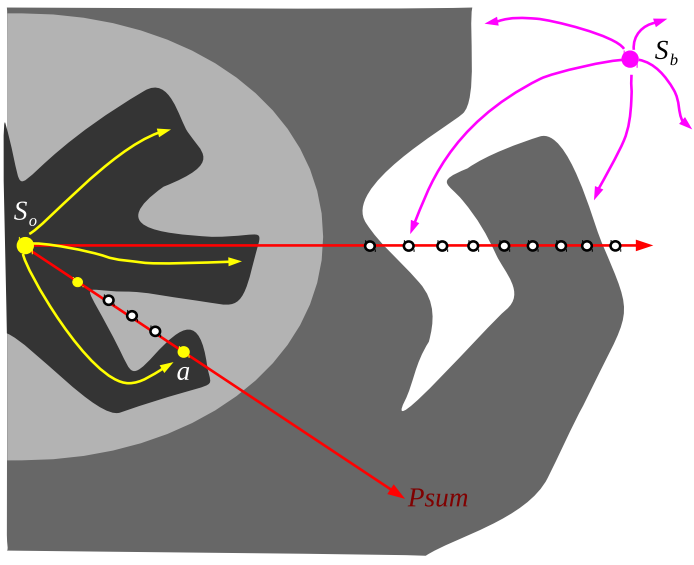

(b)

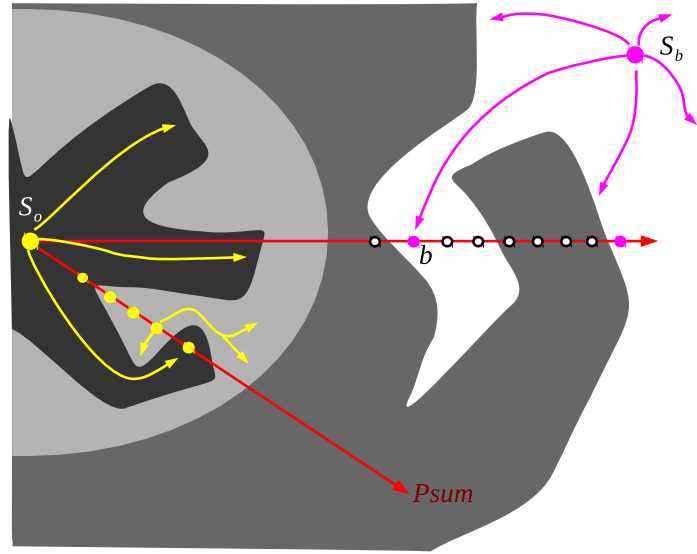

(d)

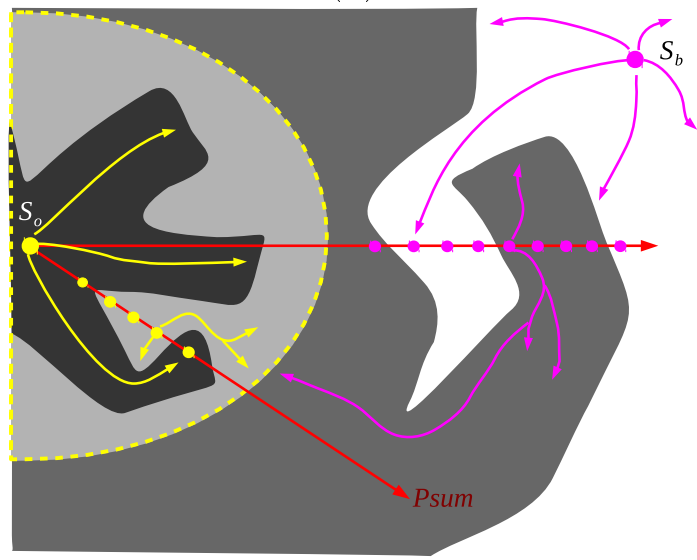

(f)

Figura 7.6: (a) Imagem sintética com três possíveis bordas de corte, onde $s_{o}$ e $s_{b}$ são sementes do objeto e fundo respectivamente, e a floresta $P_{\text {sum }}$ permite a visibilidade das restrições de forma. (b) Após o pixel a sair da fila $Q$ com rótulo 1 , temos a violação da visibilidade do pixel a em relação a sua semente mais próxima $s_{o}$. (c) O procedimento Conquistar_Caminho atribui rótulo 1 a todos seus predecessores, permitindo a expansão dos caminhos do objeto na região cinza claro. (d) O pixel b sai da fila $Q$ com rótulo de fundo, bloqueando a visibilidade dos seus descendentes em $P_{\text {sum }}$ em relação a $s_{o}$ (e) O procedimento Podar_Ārvore atribui rótulo 0 a seus descendentes, permitindo a expansão dos caminhos de fundo na região cinza escuro. (f) Resultado da segmentação (linha amarela pontilhada) com o algoritmo GSC-IFT. 


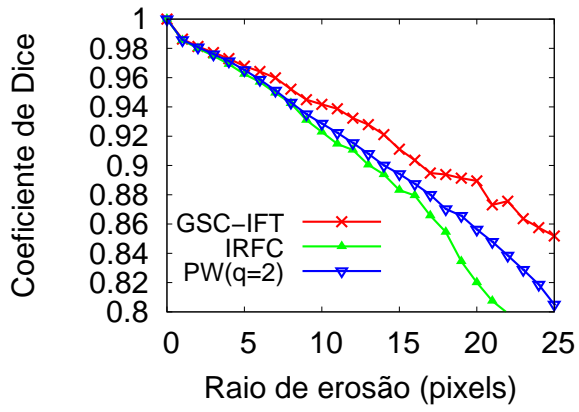

(a)

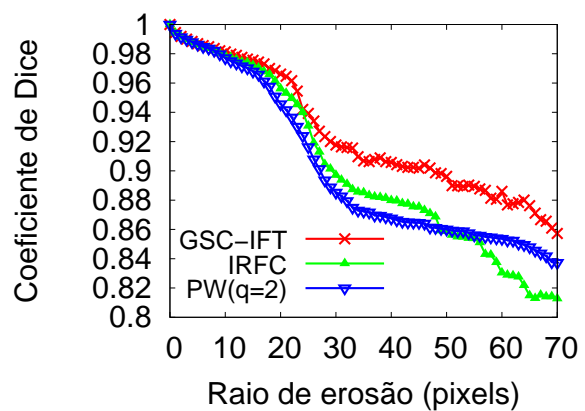

(c)

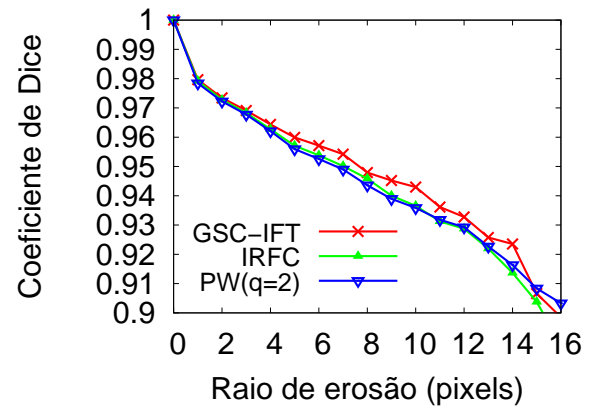

(b)

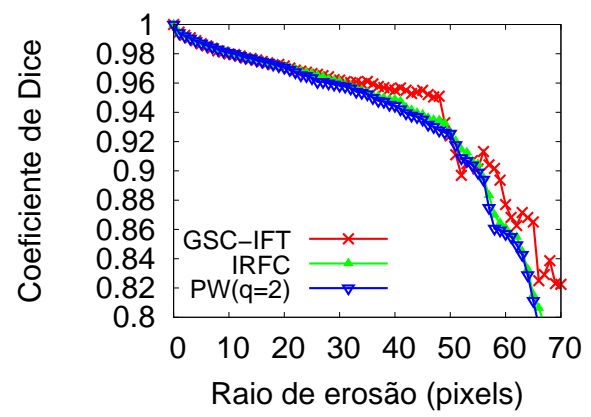

(d)

Figura 7.7: (a) Curvas de acurácia dos métodos GSC-IFT, IRFC e $P W(q=2)$ para a segmentação de: (a) calcâneo, (b) tálus, (c) fígado, e (d) mama.

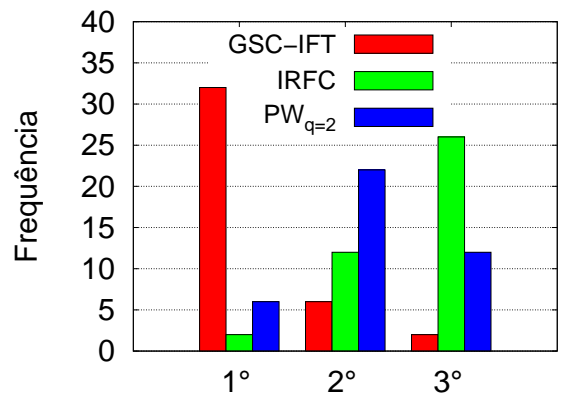

(a)

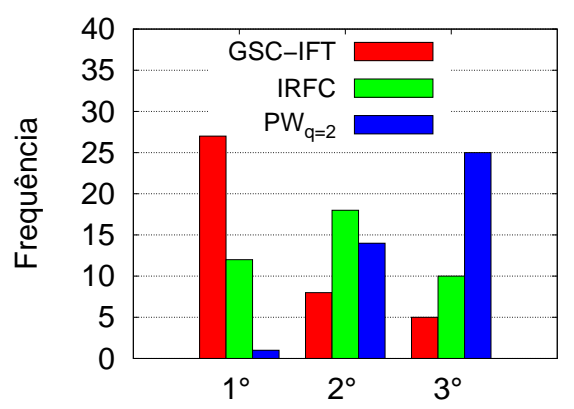

(c)

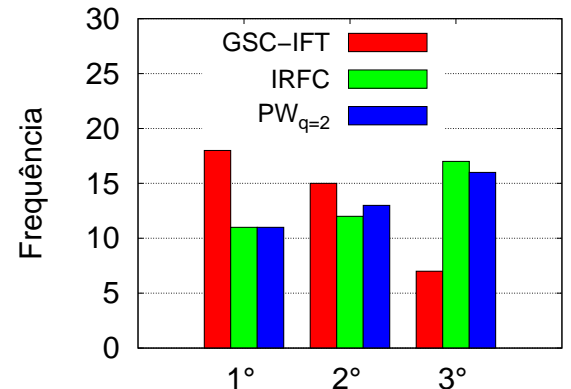

(b)

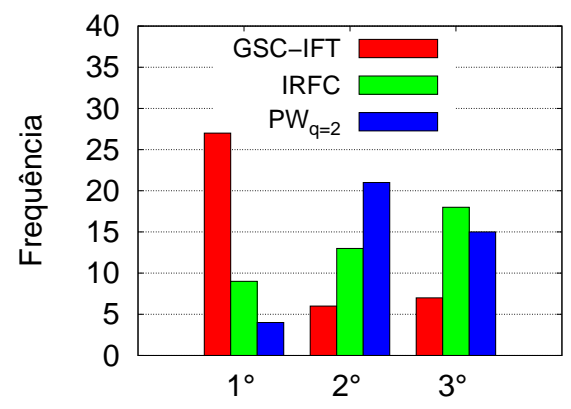

(d)

Figura 7.8: Para cada imagem individual, os métodos podem ser ranqueados de acordo a seus valores médios do coeficiente de Dice, como primeiro (o melhor), segundo, ou terceiro (o pior). Calculando a frequência para cada posição de classificação, temos uma distribuição de classificação para segmentar: (a) calcâneo, (b) tálus, (c) fígado, e (d) mama. 
Um exemplo de segmentação do osso tálus pode ser observado na Figura 7.9, demonstrando as vantagens do algoritmo GSC-IFT sobre o IRFC. Outros exemplos de segmentação como skull stripping e segmentação em imagens coloridas são apresentados nas Figuras 7.10 e 7.11, respectivamente.

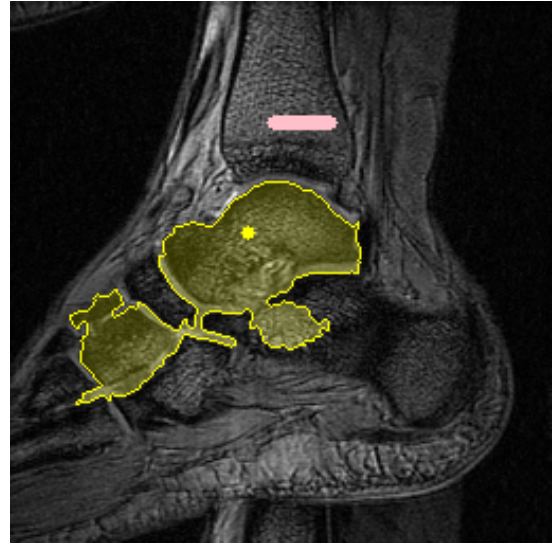

(a)

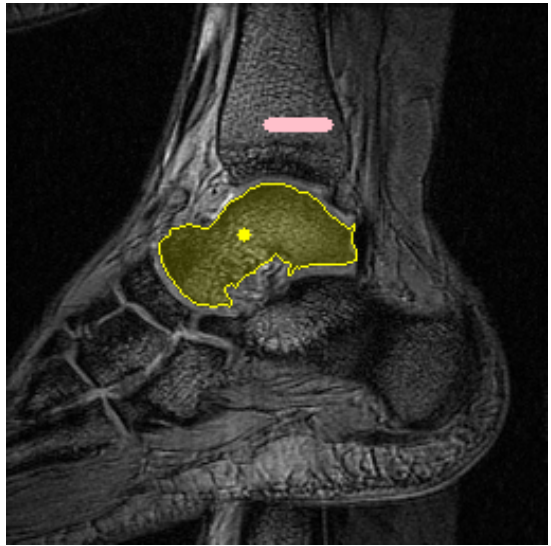

(b)

Figura 7.9: Segmentação do osso tálus: Em (a) temos o resultado do IRFC para os marcadores selecionados. Em (b) temos que um melhor resultado pode ser obtido por GSC-IFT para os mesmos marcadores.

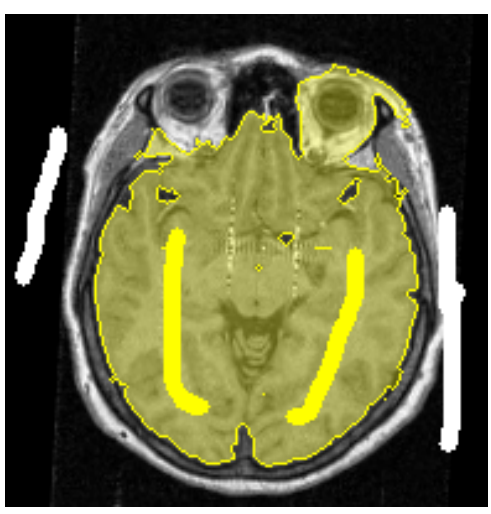

(a)

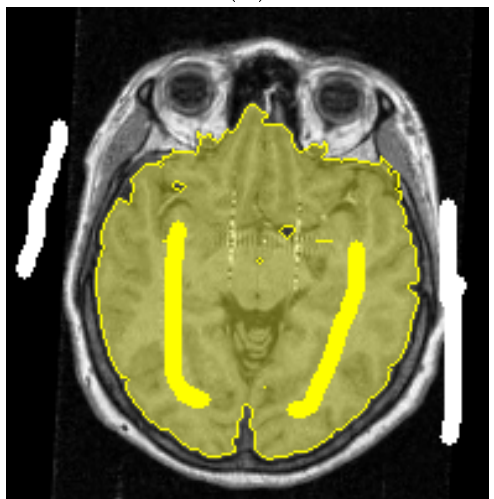

(c)

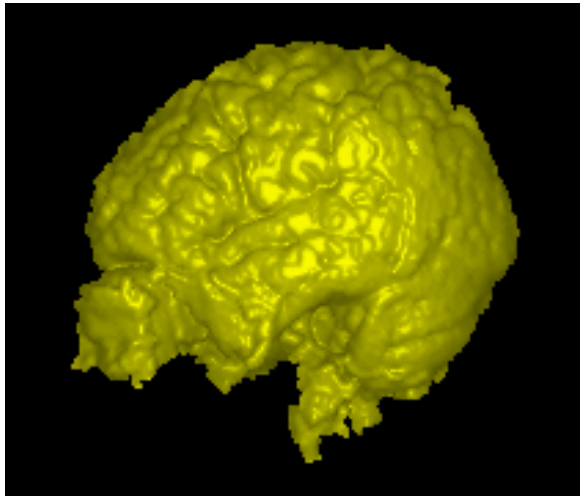

(b)

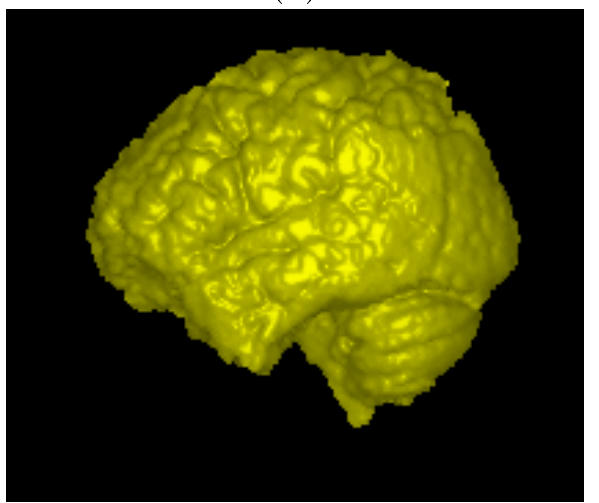

(d)

Figura 7.10: Exemplo de segmentação do cérebro (skull stripping) em 3D usando marcadores selecionados pelo usuário. Em (a) e (b) temos o resultado da segmentação por IFT-SC. Em (c) e (d) temos que um resultado melhor é obtido pela GSC-IFT $\operatorname{com} \beta=0.1$. 


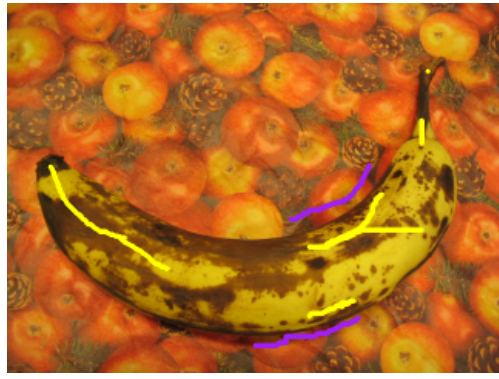

(a)

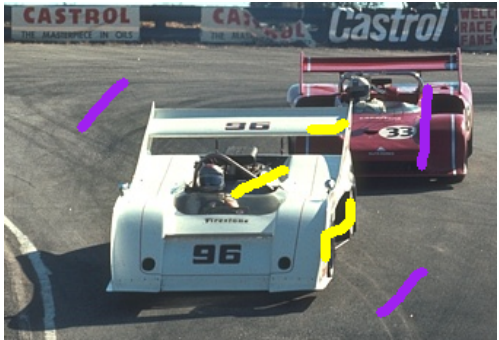

(d)

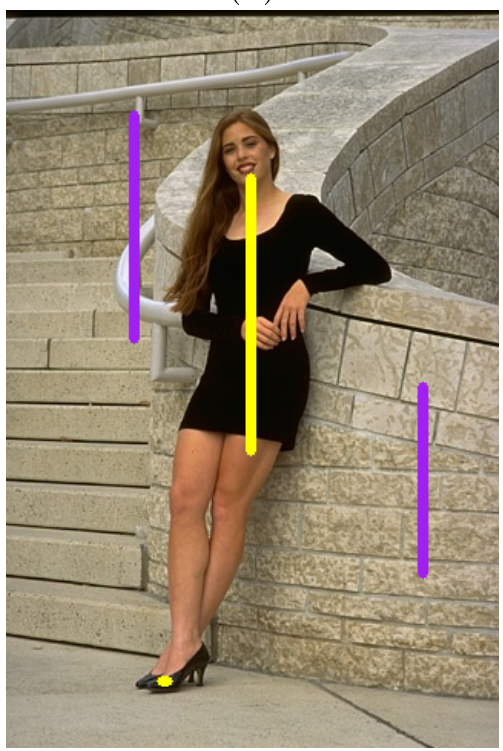

(g)

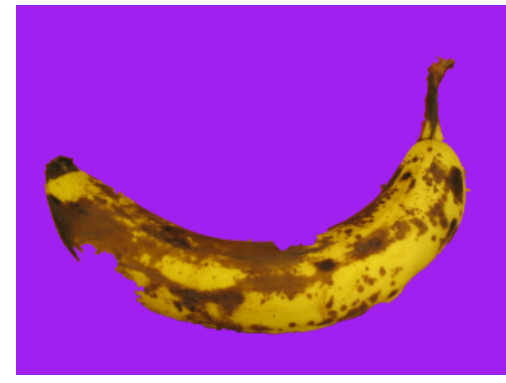

(b)

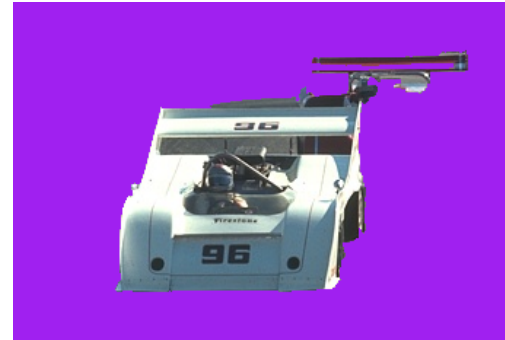

(e)

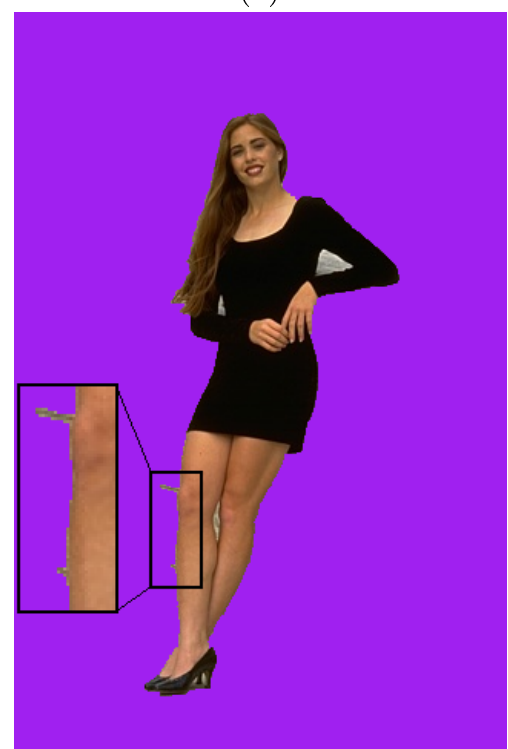

(h)

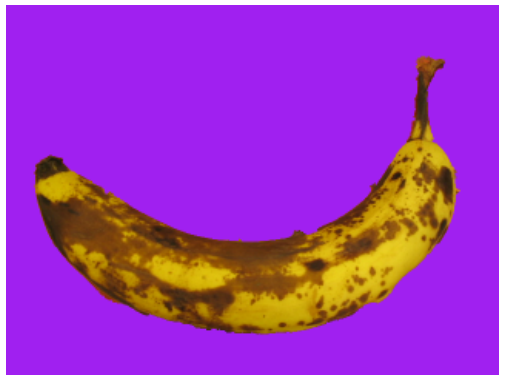

(c)

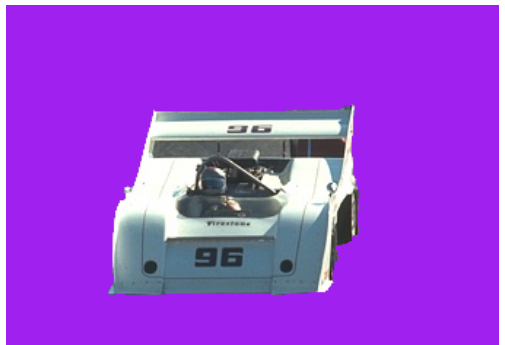

(f)

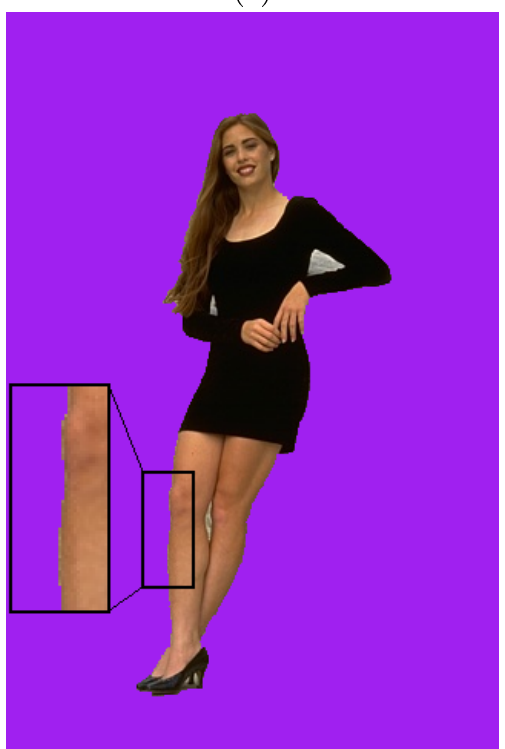

(i)

Figura 7.11: Em (a),(d) e (g) temos imagens de entrada com marcadores selecionados pelo usuário. Em (b), (e) e (h) temos o resultado da segmentação pela IFT-SC. Em (c), (f) e (i) temos o resultado da segmentação pelo GSC-IFT $(\beta=0.1)$. 


\section{Capítulo 8}

\section{Transformada imagem-floresta com tratamento simultâneo de polaridade de borda e restrições de forma}

Como vimos no Capítulo 6, o enfoque da OIFT para segmentação interativa fazendo uso das funções de conexidade não suaves $f_{i, \max }^{b k g}$ e $f_{o, \max }^{b k g}$ favorecem a segmentação de objetos que apresentam transições de pixels de escuro para claro e de claro para escuro respectivamente na sua borda. Além disso, no Capítulo 7 observamos que as restrições de forma pela convexidade geodésica em estrela (GSC) eliminam formas complexas não desejadas, melhorando a segmentação dos objetos com forma mais regular. Portanto, um tratamento simultâneo do uso da informação da polaridade de borda e das restrições de forma nos possibilitaria um maior grau de especificidade na segmentação (Figura 8.1).

Neste capítulo, nós propomos o método GSC-OIFT, o qual pode lidar simultaneamente com a orientação de borda (usando um parâmetro $\alpha$ que controla o peso dado a orientação no grafo ponderado direcionado) e com as restrições de forma (usando um parâmetro $\beta$ que controla a topologia da floresta geodésica), o que permite eliminar formas complexas não desejadas, melhorando a segmentação de objetos com forma mais regular. GSC-OIFT só requer duas vezes o tempo computacional da IFT convencional.

\subsection{Segmentação via OIFT com restrições de conexidade geodésica em estrela através das funções $f_{i, \max }^{b k g}$ e $f_{o, \max }^{b k g}$}

Considere um grafo direcionado ponderado denotado como $G=(\mathcal{I}, \xi, \omega)$, onde os pesos $\omega$ tem a configuração de acordo com a Equação 6.3. Para qualquer imagem rotulada binária $L$ (Figura 8.2) temos dois tipos de conjuntos de arestas de corte na borda do objeto, um conjunto de arestas de corte interno $\mathcal{C}_{i}$ (Equação 8.1) e um conjunto de arestas de corte externo $\mathcal{C}_{o}$ (Equação 8.2).

$$
\begin{aligned}
& \mathcal{C}_{i}(L)=\{(s, t) \in \xi \mid L(s)=0, L(t)=1\} \\
& \mathcal{C}_{o}(L)=\{(s, t) \in \xi \mid L(s)=1, L(t)=0\}
\end{aligned}
$$




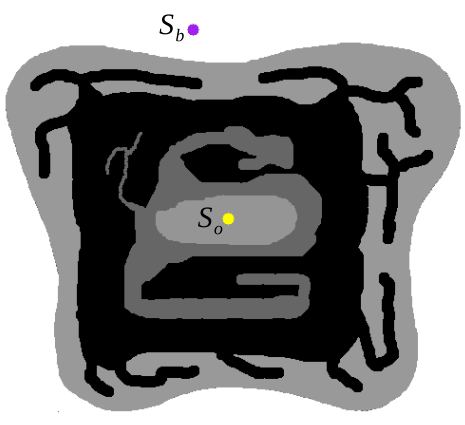

(a)

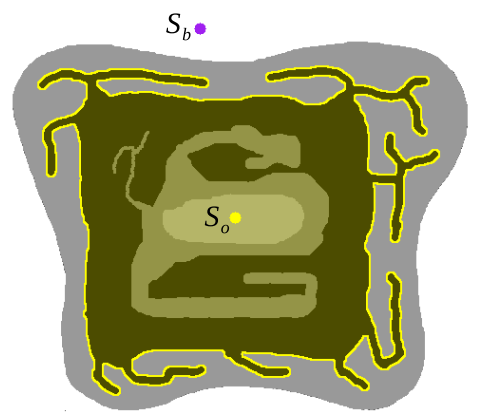

(b)

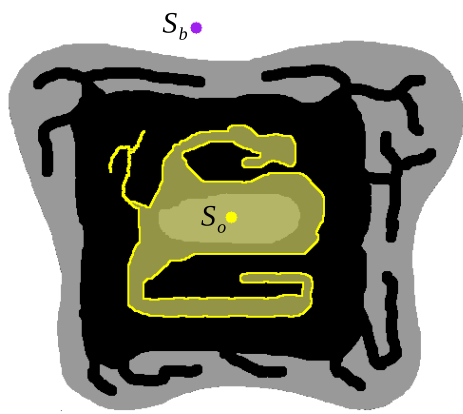

(c)

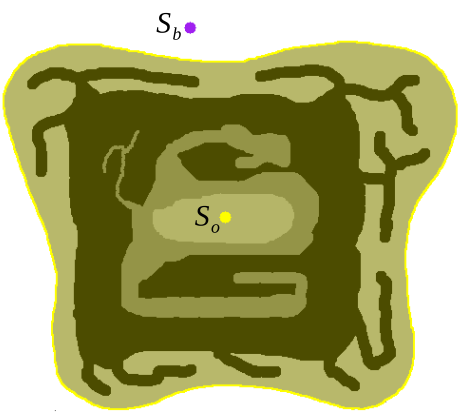

(d)

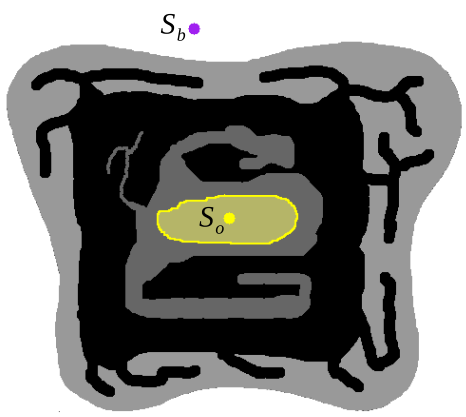

(e)

Figura 8.1: (a) Uma imagem original com marcadores $S_{o}$ e $S_{b}$. (b) Resultado da segmentação com IFT. (c) Resultado da segmentação com OIFT. (d) Resultado da segmentação com GSC-IFT. (e) Resultado da segmentação considerando simultaneamente a polaridade de borda e as restrições de forma (GSC-OIFT).

Além disso, considere uma floresta $P_{\text {sum }}$ calculada pelo algoritmo da IFT com a função de soma $f_{\text {sum }}$, usando somente o conjunto de sementes internas $\mathcal{S}_{o}$ para o grafo direcionado ponderado $G$, e dois conjuntos de arestas em $P_{\text {sum }}, \xi_{P_{\text {sum }}}^{i}$ e $\xi_{P_{\text {sum }}}^{o}$ definidos pelas Equações 8.3 e 8.4 respectivamente.

$$
\begin{aligned}
& \xi_{P_{\text {sum }}}^{i}=\left\{(s, t) \in \xi \mid s=P_{\text {sum }}(t)\right\} \\
& \xi_{P_{\text {sum }}}^{o}=\left\{(s, t) \in \xi \mid t=P_{\text {sum }}(s)\right\}
\end{aligned}
$$

Assim, se uma aresta $(s, t) \in \xi$ está na interseção dos conjuntos $\mathcal{C}_{i}(L)$ e $\xi_{P_{\text {sum }}}^{i}$ teremos uma violação da restrição de convexidade em forma de estrela (Figura 8.2(a)), dado que o pixel $t$ pertence ao objeto e não seria visível ao centro de estrela $\mathcal{S}_{0}$. Uma situação similar acontece para uma aresta $(s, t) \in \xi$ que está em $\mathcal{C}_{o}(L) \cap \xi_{P_{\text {sum }}}^{o}$ (Figura 8.2(b)).

Baseados na identificação desse tipo de arestas, que violam a restrição de convexidade geodésica em estrela, propomos o Lema 1 e o Teorema 4, cujas provas estão apresentadas no Apêndice E.

Nesse capítulo, adotamos as seguintes definições para as funções de energia interna $\left(E_{i}\right)$ e externa $\left(E_{o}\right)$ dadas pelas Equações 8.5 e 8.6 respectivamente.

$$
\begin{aligned}
& E_{i}(L, G=(\mathcal{I}, \xi, \omega))=\min _{(s, t) \in \mathcal{C}_{i}(L)} \omega(s, t) \\
& E_{o}(L, G=(\mathcal{I}, \xi, \omega))=\min _{(s, t) \in \mathcal{C}_{o}(L)} \omega(s, t)
\end{aligned}
$$

Nosso objetivo é restringir a busca de resultados ótimos, que maximizam as medidas de corte 


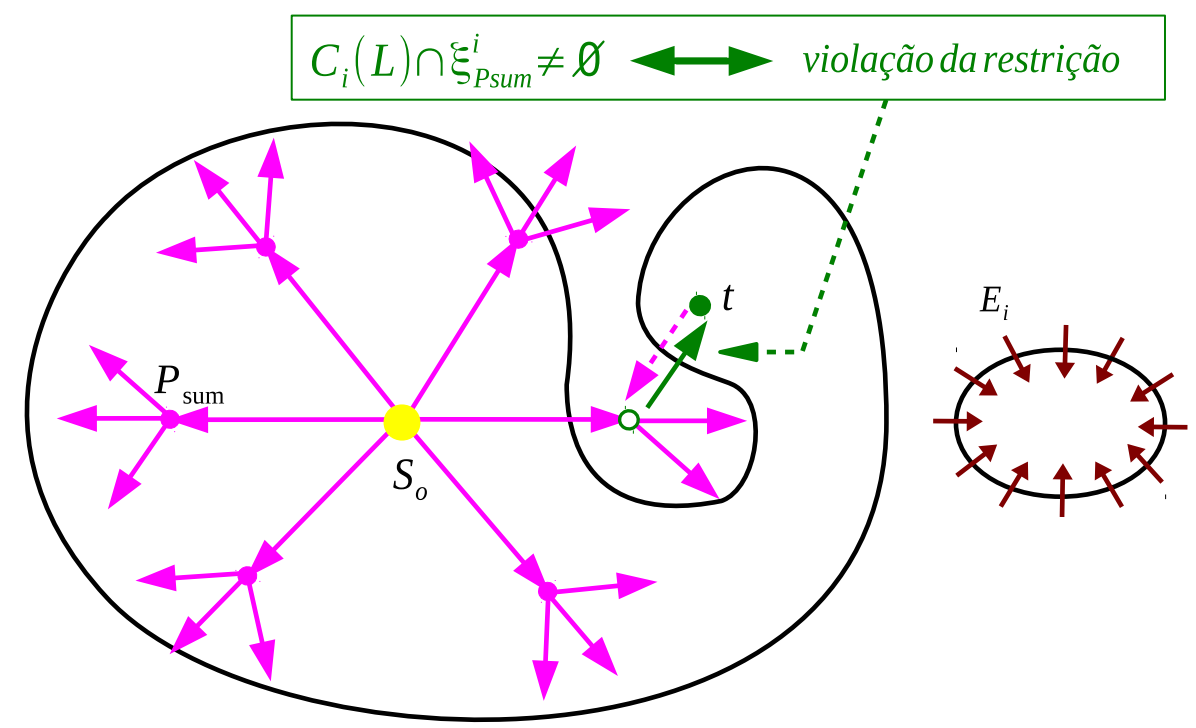

(a)

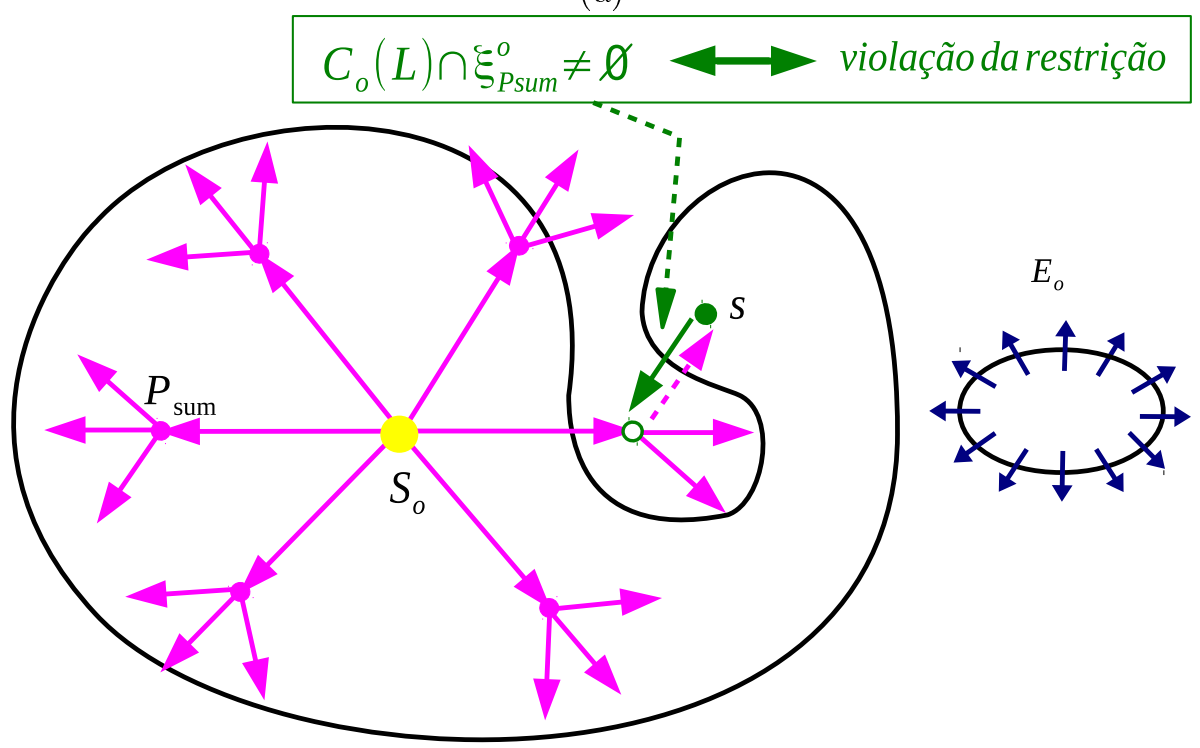

(b)

Figura 8.2: Imagem rotulada $L$ com rótulo $L(s)=1$ se o pixel s pertence ao objeto e rótulo $L(s)=0$ se pertence ao fundo. Em (a) é mostrada a violaçâo da restrição de convexidade geodésica em estrela quando existe uma aresta $(s, t) \in\left(\mathcal{C}_{i}(L) \cap \xi_{P \text { sum }}^{i}\right)$. Em (b) é mostrada a violação da restrição de convexidade geodésica em estrela quando existe uma aresta $(s, t) \in\left(\mathcal{C}_{o}(L) \cap \xi_{P_{\text {sum }}}^{o}\right)$.

em grafo $E_{i}$ e $E_{o}$, somente para segmentações que satisfazem restrições de convexidade geodésica em estrela.

Lema 1 Para uma dada segmentação $L$, nós temos $\mathcal{C}_{o}(L) \cap \xi_{P_{\text {sum }}}^{o} \neq \emptyset$, se e somente se existe uma violação da restrição de convexidade geodésica em estrela. Nós temos $\mathcal{C}_{i}(L) \cap \xi_{P_{\text {sum }}}^{i} \neq \emptyset$, se e somente se existe uma violação da restrição geodésica em estrela.

Teorema 4 (Otimalidade da borda de corte interno/externo) Para um dado grafo ponderado $G=(\mathcal{I}, \xi, \omega)$, considere um grafo ponderado modificado $G^{\prime}=\left(\mathcal{I}, \xi, \omega^{\prime}\right)$, com pesos $\omega^{\prime}(s, t)=$ $-\infty$ para todo $(s, t) \in \xi_{P_{\text {sum }}}^{o}$, e $\omega^{\prime}(s, t)=\omega(s, t)$ caso contrário. Para dois conjuntos $\mathcal{S}_{o}$ e $\mathcal{S}_{b}$ de sementes dados, a segmentação calculada sobre $G^{\prime}$ pelo algoritmo da IFT (Algoritmo 1) para a função $f_{o, \max }^{b k g}$ define um corte ótimo no grafo original $G$, que maximiza $E_{o}(L, G)$ entre todos os resultados 

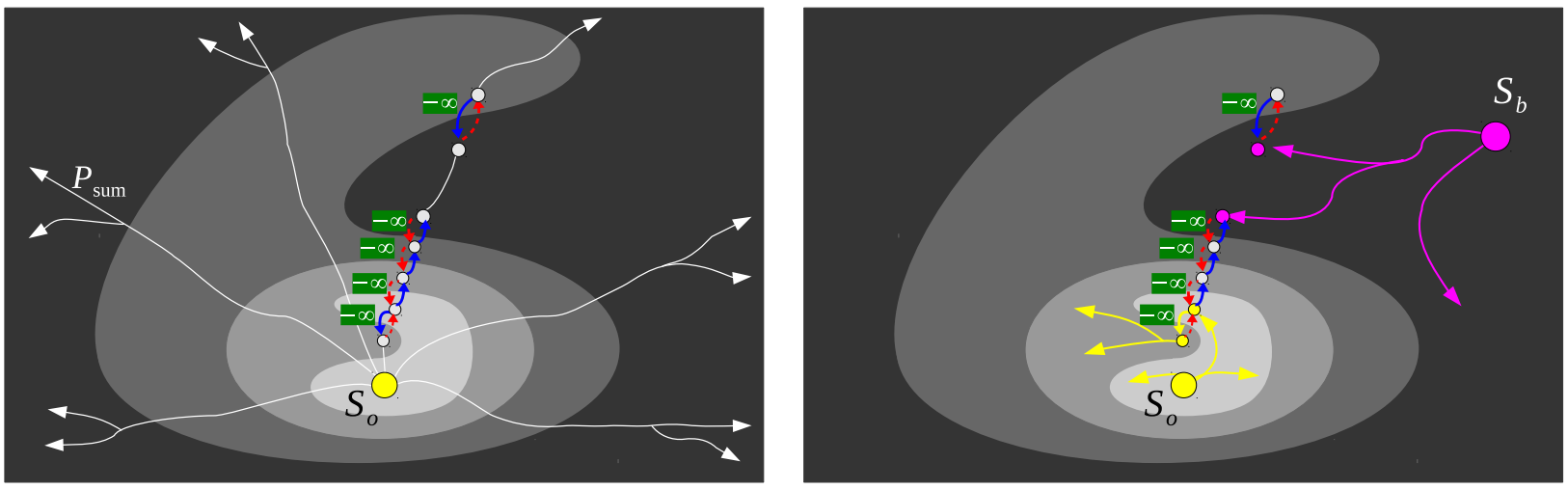

(a)

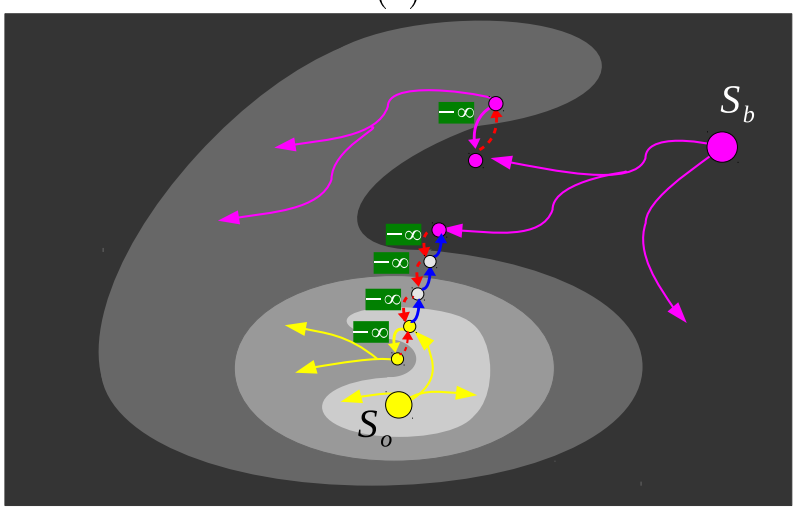

(c) (b)

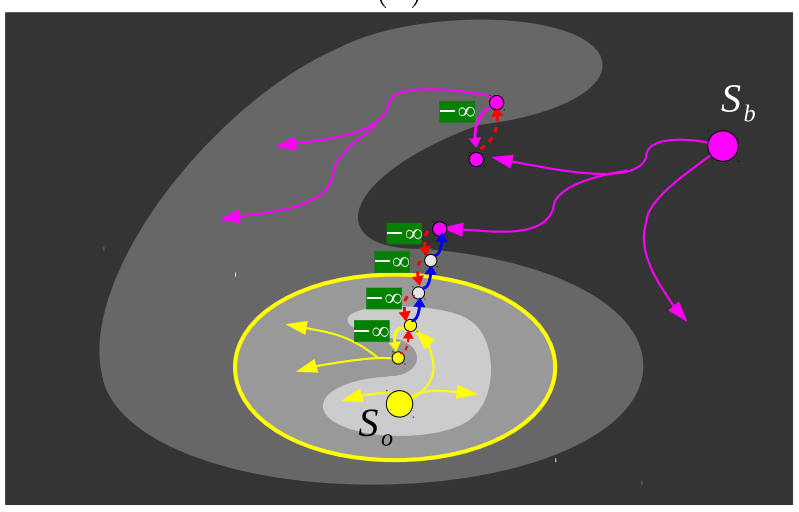

(d)

Figura 8.3: (a) Imagem sintética com três possíveis bordas de corte, com mesma orientação (claro para escuro) floresta $P_{\text {sum }}$ e semente interna $s_{o}$ (centro de estrela). As arestas com valor $-\infty$ representam arestas $(s, t) \in \xi_{P_{\text {sum }}}^{o} q u e$ violam as restrições de forma pela GSC. As setas azuis representam cortes externos nas bordas e as vermelhas (setas pontilhadas) cortes internos. (b) Pixels são conquistados pela semente interna $s_{o}$. (c) Pixels são conquistados pela semente externa $s_{b}$. (d) Resultado da segmentação (linha amarela) pela $G S C-O I F T$ com $f_{o, \max }^{b k g}$.

possíveis de segmentação satisfazendo as restrições de forma pela convexidade geodésica em estrela, e as restriçôes de sementes.

Similarmente, a segmentação calculada pelo algoritmo da IFT (Algoritmo 1) para a função $f_{i, \max }^{b k g}$, sobre o grafo modificado $G^{\prime}=\left(\mathcal{I}, \xi, \omega^{\prime}\right) ; \operatorname{com}$ pesos $\omega^{\prime}(s, t)=-\infty$ para todo $(s, t) \in \xi_{P_{\text {sum }}}^{i}, e$ $\omega^{\prime}(s, t)=\omega(s, t)$ caso contrário; define um corte ótimo no grafo original $G$, que maximiza $E_{i}(L, G)$ entre todos os resultados possíveis de segmentação satisfazendo as restrições de forma pela convexidade geodésica em estrela, e as restrições de sementes.

Na Figura 8.3 é mostrado um exemplo do método GSC-OIFT com a função não suave $f_{o, \max }^{b k g}$.

\subsubsection{Resultados experimentais}

Nos experimentos foram usadas um total de 40 fatias de imagens de 10 estudos torácicos em TC, para segmentar o fígado, obtidas de um especialista do departamento de radiologia da Universidade da Pensilvânia. Os resultados mostraram uma melhora de acurácia significativa. As curvas médias 
de acurácia para os algoritmos avaliados (isto é, IRFC representado pela IFT com $f_{\text {max }}$, power watershed [Couprie et al. (2010)], OIFT, GSC-IFT e GSC-OIFT) são apresentadas na Figura 8.4, assumindo diferentes conjuntos de sementes obtidos pela erosão do gabarito.

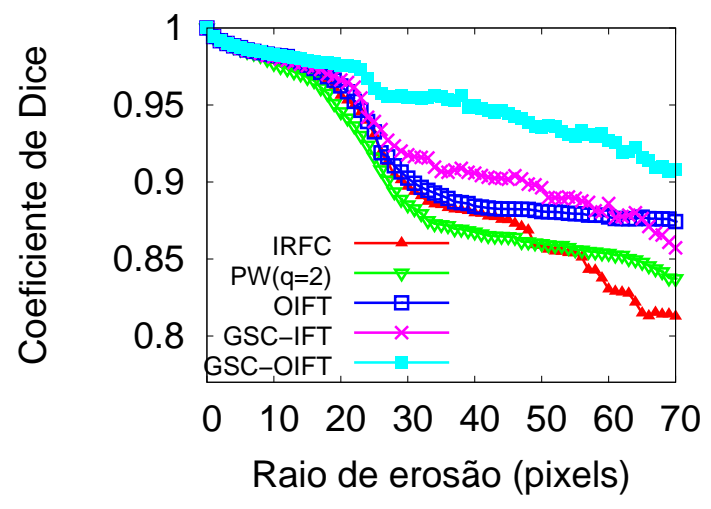

(a)

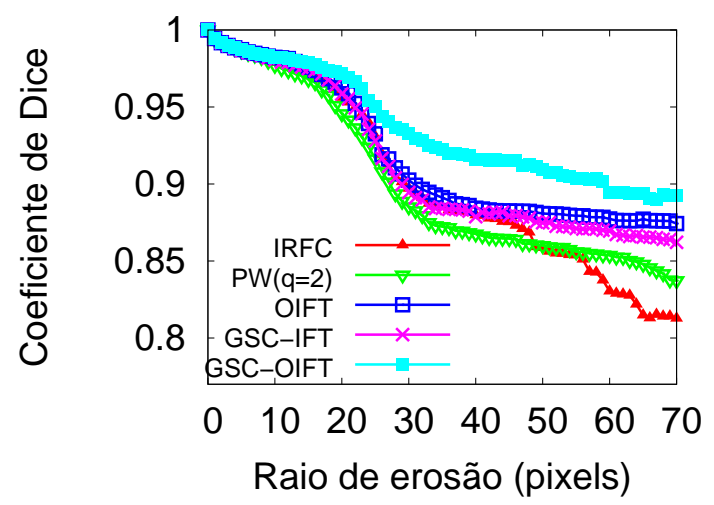

(c)

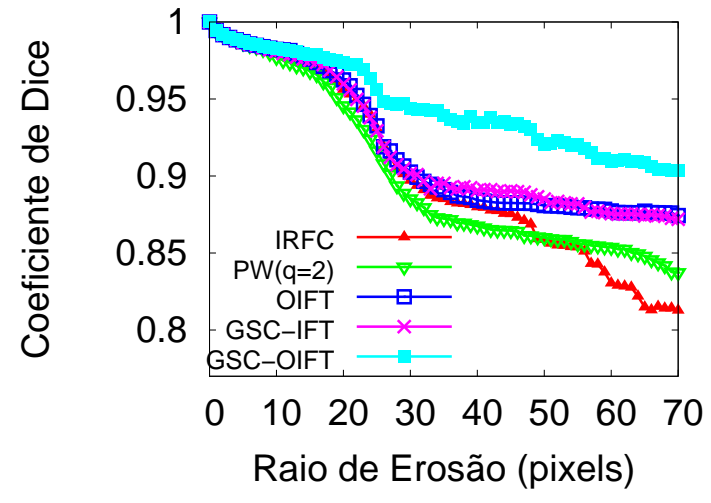

(b)

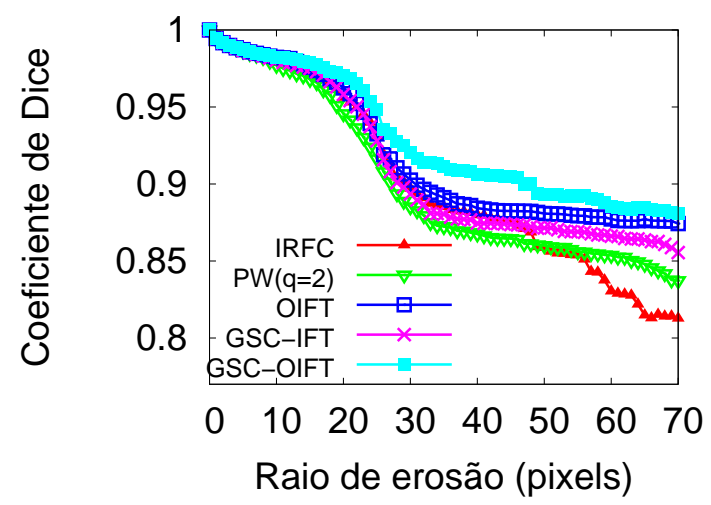

(d)

Figura 8.4: (a) Curvas médias de acurácia para a segmentação do fígado para diferentes valores de $\beta$ : (a) $\beta=0.0$, (b) $\beta=0.2$, (c) $\beta=0.5$, e (d) $\beta=0.7$.

Um exemplo de segmentação com os métodos IRFC, OIFT $\left(f_{o, \text { max }}^{b k g}\right)$, GSC-IFT e GSC-OIFT é apresentado na Figura 8.5. Na Figura 8.6 é mostrado um exemplo de aplicação para o problema de skull stripping em imagens 3D de RM, onde podemos observar os resultados da segmentação dos métodos IRFC, GSC-IFT e GSC-OIFT. 


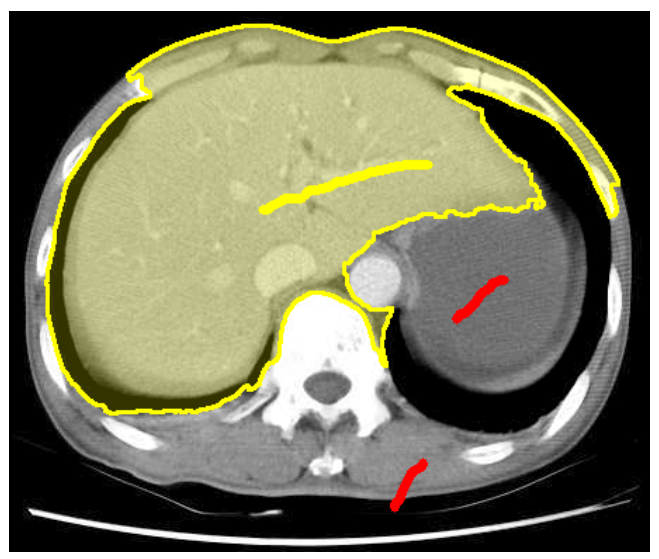

(a)

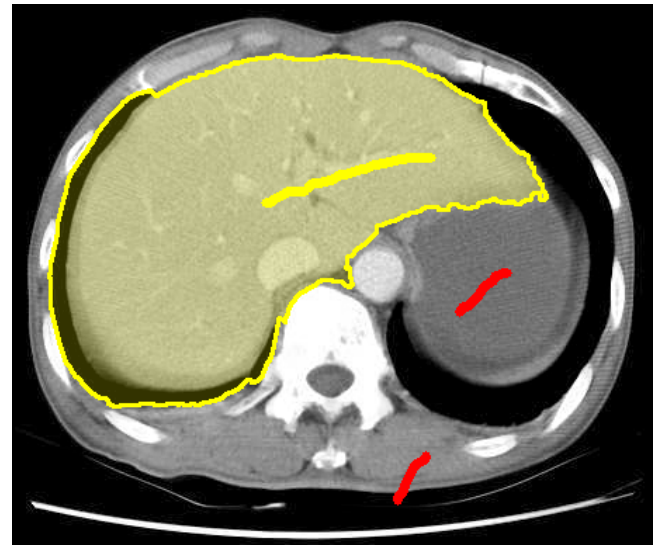

(c)

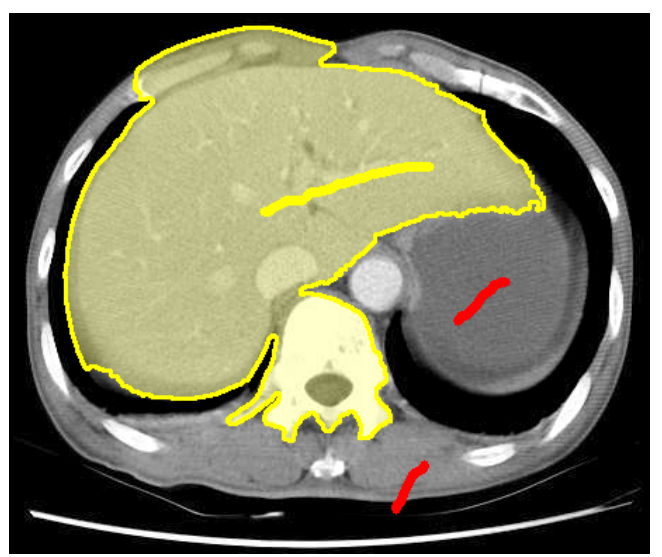

(b)

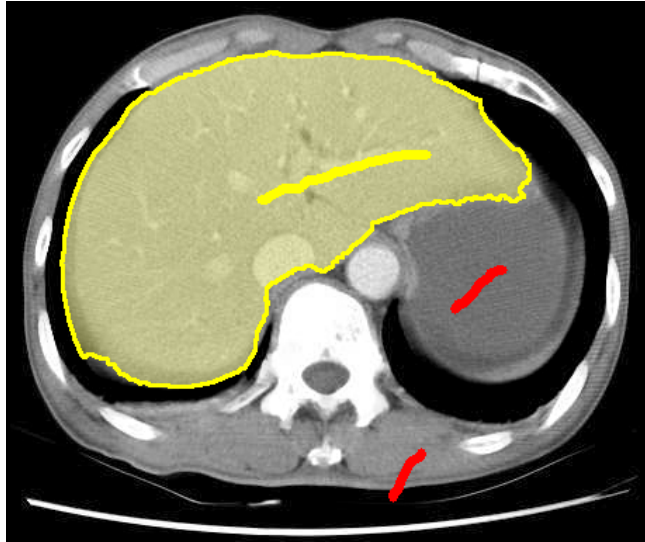

(d)

Figura 8.5: Resultados para marcadores selecionados pelo usuário: (a) IRFC (IFT com $f_{\max }$ ), (b) OIFT $\left(f_{o, \max }^{b k g} \operatorname{com} \alpha=0.5\right)$, (c) GSC-IFT $(\beta=0.7, \alpha=0.0)$, e (d) GSC-OIFT $(\beta=0.7, \alpha=0.5)$.

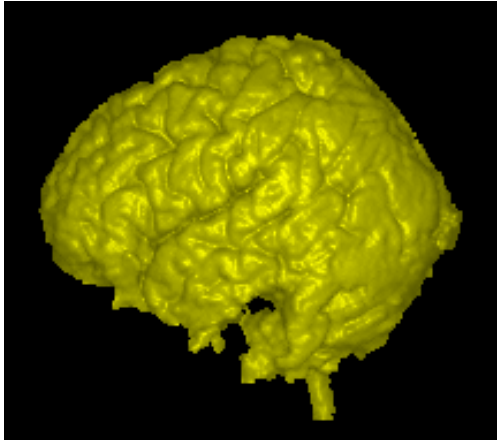

(a)

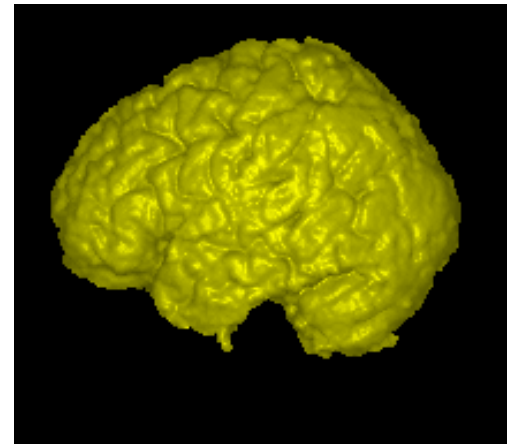

(b)

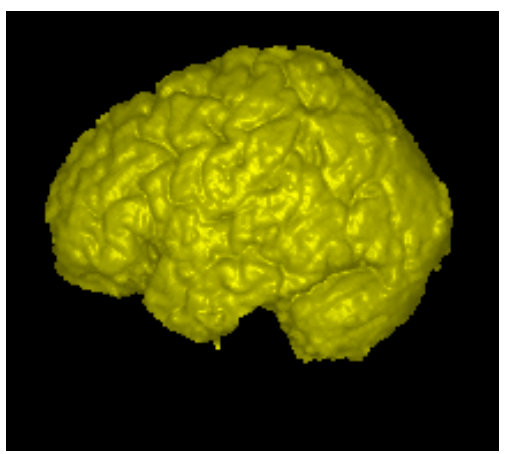

(c)

Figura 8.6: Exemplo de skull stripping $3 D$ em RM: (a) IRFC (IFT com $\left.f_{\max }\right)$, (b) GSC-IFT ( $\beta=0.3$, $\alpha=0.0)$, e (c) GSC-OIFT ( $\beta=0.3, \alpha=0.5)$, para os mesmos marcadores selecionados pelo usuário. 


\section{Capítulo 9}

\section{Conclusões}

\subsection{Considerações Finais}

Nosso objetivo principal foi realizar um estudo sobre o uso das funções de conexidade não suaves no arcabouço da IFT. Esse estudo culminou com a proposta de uma classificação das funções de conexidade não suaves, evidenciando um subconjunto com propriedades teóricas interessantes, de modo que algumas funções pertencentes a essa região mostraram bons resultados na segmentação interativa de imagens com forte inomogeneidade de campo. Também, neste estudo, três novos métodos de segmentação interativa de imagens foram introduzidos: (i) Transformada imagem-floresta orientada (OIFT), que favorece a segmentação de imagens que apresentam objetos com transições de pixels na borda de claro para escuro ou de escuro para claro. (ii) IFT com convexidade geodésica em estrela (GSC-IFT), que permite segmentar imagens que apresentam objetos com forma regular, não permitindo resultados de formas complexas. (iii) OIFT com convexidade geodésica em estrela (GSC-OIFT), que combina as vantagens da OIFT e da GSC-IFT favorecendo a segmentação de imagens que têm objetos de forma regular e que apresentam orientação bem definida nas suas bordas (de claro para escuro ou vice-versa).

A GSC-OIFT engloba de certo modo os métodos anteriores OIFT e GSC-IFT (bem como a IFT convencional com $f_{\max }$ ) de acordo com a configuração dos seus parâmetros $\alpha$ e $\beta$. Ela poderia também ser implementada com base nas funções $f_{i, \omega}$ e $f_{o, \omega}$, de modo a permitir um melhor tratamento no caso de empates.

Note porém que as funções adaptativas apresentadas no Capítulo 5 não podem ser reduzidas ao cálculo de uma GSC-OIFT. Dado que o desempenho desses métodos depende do tipo de imagem, temos que todos são igualmente relevantes. A tabela 9.1 apresenta uma classificação do uso dos métodos propostos neste texto, de acordo com as características específicas da imagem.

\subsection{Contribuições}

Além das contribuições teóricas em métodos de segmentação baseados em grafos, o projeto também contribuiu com o desenvolvimento de um aplicativo de apoio para a pesquisa no campo da medicina.

Vários trabalhos desenvolvidos pelo orientador e seus colaboradores no contexto do programa FAPESP-CInApCe ${ }^{1}$ (em colaboração com os professores do Departamento de Neurologia da Fa-

\footnotetext{
${ }^{1}$ Projeto que visava a criação de uma rede de pesquisa multidisciplinar para adquirir tecnologia de ponta e estudar
} 


\begin{tabular}{|l|l|}
\hline Característica na imagem & Método proposto \\
\hline Presença de efeitos de inomogeneidade. & IFT com $f_{\Sigma \mid \Delta \backslash \mathcal{I} \text {, ou } f_{\uparrow}^{l e x} .}^{\text {lex }}$ \\
\hline $\begin{array}{l}\text { Transição de pixels na borda do objeto de escuro para claro } \\
\text { sem restrição de forma. }\end{array}$ & $\begin{array}{l}\text { OIFT com } f_{i, \max }^{b k g}(\alpha=0.5), \\
\text { OIFT com } f_{i, \omega}(\alpha>=0.5), \text { ou } \\
\text { GSC-OIFT com } f_{i, \omega}(\beta>=1.0 \text { e } \alpha>=0.5) .\end{array}$ \\
\hline $\begin{array}{l}\text { Transição de pixels na borda do objeto de claro para escuro } \\
\text { sem restrição de forma. }\end{array}$ & $\begin{array}{l}\text { OIFT com } f_{o, \max }^{b k g}(\alpha>=0.5), \\
\text { OIFT com } f_{o, \omega}(\alpha>=0.5), \text { ou } \\
\text { GSC-OIFT com } f_{o, \omega}(\beta>=1.0 \text { e } \alpha>=0.5) .\end{array}$ \\
\hline $\begin{array}{l}\text { Objeto de forma estritamente regular (convexo em estrela) } \\
\text { e transição de pixels na borda de escuro para claro. }\end{array}$ & GSC-OIFT com $f_{i, \max }^{b k g}(\beta=0$ e $\alpha>=0.5)$. \\
\hline $\begin{array}{l}\text { Objeto de forma estritamente regular (convexo em estrela) } \\
\text { e transição de pixels na borda de claro para escuro. }\end{array}$ & GSC-OIFT com $f_{o, \max }^{b k g}(\beta=0$ e $\alpha>=0.5)$. \\
\hline $\begin{array}{l}\text { Objeto de forma regular (convexo em estrela) } \\
\text { permitindo um certo grau de variabilidade na sua forma, } \\
\text { e transição de pixels na borda de escuro para claro. }\end{array}$ & GSC-OIFT com $f_{i, \max }^{b k g}(\beta>0$ e $\alpha>=0.5)$. \\
\hline $\begin{array}{l}\text { Objeto de forma regular (convexo em estrela) } \\
\text { permitindo um certo grau de variabilidade na sua forma, } \\
\text { e transição de pixels na borda de claro para escuro. }\end{array}$ & GSC-OIFT com $f_{o, \max }^{b k g}(\beta>0$ e $\alpha>=0.5)$. \\
\hline
\end{tabular}

Tabela 9.1: Relação dos métodos propostos e o tipo de imagem a ser segmentada.

culdade de Ciências Médicas da UNICAMP), têm sido agregados a uma ferramenta computacional chamada Brain Image Analyzer (BIA), que foi usada pelos médicos; por exemplo, em estudos envolvendo volumetria de hemisfério cerebrais e cerebelo em indivíduos com epilepsia do lobo temporal mesial e antecedente familiar para epilepsia (FAPESP 12/2008-11/2010). Nesse contexto, os resultados do presente projeto foram incorporados na ferramenta BIA, como módulos de extensão, favorecendo a segmentação interativa de imagens volumétricas de ressonância magnética adquiridas com um scanner de 3T. O projeto também contribuiu em um projeto FINEP(1266/13) em engenharia biomédica, no projeto universal CNPq (486083/2013-6), e no projeto FAPESP(2012/06911-2).

\subsection{Sugestões para pesquisas futuras}

As técnicas desenvolvidas podem também favorecer métodos automáticos [Miranda et al. (2008b, 2009)] na segmentação de imagens. Esses métodos fornecem conjuntos automáticos de sementes que podem ser combinados com as técnicas propostas neste documento. Também poderia combinar-se as funções de conexidade não suaves com modelos estatísticos para segmentação automática 3D.

Uma outra sugestão seria explorar outras funções de conexidade não suaves de regiões não exploradas do diagrama.

Por outro lado, a IFT pode ser estendida do domínio da imagem para o domínio do espaço de características [Papa et al. (2009)], sendo possível estender o estudo das funções de conexidade não suaves na classificação de padrões.

a dinâmica cerebral associada a epilepsias e outras doenças degenerativas [Falcão et al. (2008)]. 


\section{Apêndice A}

\section{Funções de conexidade não suaves}

Neste apêndice apresentamos as definições das funções de conexidade não suaves $f_{\sum \max }, f_{\uparrow}$, $f_{\max |\triangle I|}, f_{\sum|\triangle I|}, f_{I}$, e $f_{\sum \max }^{l e x}$; incluindo uma prova formal da sua posição no diagrama apresentado na Figura 4.1.

\section{A.1 Função soma das arestas máximas $\left(f_{\sum \max }\right)$}

Seja a função $f_{\Sigma \max }$ definida por:

$$
\begin{aligned}
f_{\Sigma \max }\left(\pi_{t}=\langle t\rangle\right) & = \begin{cases}0, & \text { se } t \in \mathcal{S}, \\
+\infty, & \text { caso contrário. }\end{cases} \\
f_{\Sigma \max }\left(\pi_{t}=\pi_{s} \cdot\langle s, t\rangle\right) & =f_{\Sigma \max }\left(\pi_{s}\right)+f_{\max }\left(\pi_{t}\right),
\end{aligned}
$$

onde $\mathcal{S}$ é o conjunto de sementes.

A função $f_{\Sigma \max }$ é uma função não suave que pertence ao subconjunto $C_{1} \backslash\left(C_{2} \bigcup C_{4}\right)$ no diagrama de classificação de funções de conexidade (Figura 4.1), de modo que somente satisfaz a condição $C 1$ de suavidade (Subseção 3.1.3).

\section{Prova:}

C1. Existe um caminho ótimo $\pi_{t}=\pi_{s} \cdot\langle s, t\rangle$, tal que $f_{\Sigma \max }\left(\pi_{s}\right) \leq f_{\Sigma \max }\left(\pi_{t}\right)$.

Para $\omega(s, t) \geq 0$ nós temos que

$$
f_{\max }\left(\pi_{t}\right)=\max \left\{f_{\max }\left(\pi_{s}\right), \omega(s, t)\right\} \geq 0,
$$

então

$$
f_{\Sigma \max }\left(\pi_{s}\right) \leq f_{\Sigma \max }\left(\pi_{s}\right)+f_{\max }\left(\pi_{t}\right) .
$$

Assim, da Equação A.1 nós obtemos

$$
f_{\Sigma \max }\left(\pi_{s}\right) \leq f_{\Sigma \max }\left(\pi_{t}\right) .
$$


Portanto, $f_{\Sigma \max }$ satisfaz esta condição.

C2. Existe um caminho ótimo $\pi_{t}=\pi_{s} \cdot\langle s, t\rangle$, onde $\pi_{s}$ é ótimo.

A prova que $f_{\Sigma \max }$ viola a condição $C 2$ pode ser demonstrada pelo seguinte contra exemplo. Na Figura A.1 para um caminho ótimo $\pi_{t}=\pi_{s} \cdot\langle s, t\rangle$, nós temos que $\pi_{s}$ não é um caminho ótimo para $s$. Pois, existe outro caminho ótimo $\pi_{s}^{\prime}$ que oferece um melhor custo a $s$ do que $\pi_{s}$, isto é,

$$
f_{\Sigma \max }\left(\pi_{s}^{\prime}\right)=9 \leq f_{\Sigma \max }\left(\pi_{s}\right)=10
$$

Como $\pi_{t}^{\prime}$ não é ótimo, então a condição $C 2$ não pode ser verificada para $f_{\Sigma \max }$.

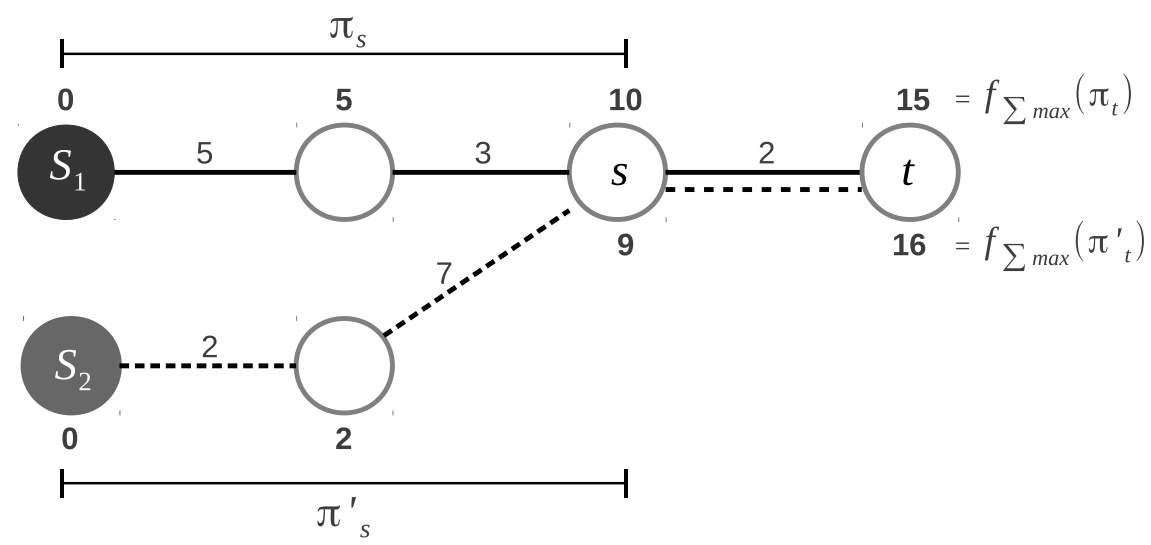

Figura A.1: Contra exemplo da segunda e terceira condições (C2 e C3) para a função $f_{\Sigma \max }$, onde $\pi_{t}=$ $\pi_{s} \cdot\langle s, t\rangle$ é um caminho ótimo e $\pi_{t}^{\prime}=\pi_{s}^{\prime} \cdot\langle s, t\rangle$ é um caminho não ótimo, a partir das sementes $S_{1}$ e $S_{2}$ respectivamente.

C3. Para qualquer caminho ótimo $\pi_{s}^{\prime}$ com destino em $s, f_{\Sigma \max }\left(\pi_{s}^{\prime} \cdot\langle s, t\rangle\right)=f_{\Sigma \max }\left(\pi_{t}\right)$.

Na Figura A.2 nós podemos observar que

$$
f_{\Sigma \max }\left(\pi_{s}^{\prime} \cdot\langle s, t\rangle\right)=16 \neq f_{\Sigma \max }\left(\pi_{t}\right)=15
$$

Então, a condição $C 3$ é violada.

C4. Para quaisquer caminhos $\pi_{s}$ e $\pi_{s}^{\prime}$ com destino em $s$, se $f_{\Sigma \max }\left(\pi_{s}\right)=f_{\Sigma \max }\left(\pi_{s}^{\prime}\right)$, então nós temos $f_{\Sigma \max }\left(\pi_{s} \cdot\langle s, t\rangle\right)=f_{\Sigma \max }\left(\pi_{s}^{\prime} \cdot\langle s, t\rangle\right)$.

Na Figura A.2, para os caminhos ótimos $\pi_{s}$ e $\pi_{s}^{\prime}$ nós temos

$$
f_{\Sigma \max }\left(\pi_{s} \cdot\langle s, t\rangle\right)=15 \neq f_{\Sigma \max }\left(\pi_{s}^{\prime} \cdot\langle s, t\rangle\right)=16 .
$$

Assim, claramente nós temos que $f_{\Sigma \max }$ não satisfaz a condição $C 4$.

Portanto, como a função $f_{\Sigma \max }$ somente satisfaz a condição $C 1$ de suavidade (Subseção 3.1.3), podemos concluir que $f_{\Sigma \max }$ é uma função não suave e $f_{\Sigma \max } \in C_{1} \backslash\left(C_{2} \cup C_{4}\right)$ no diagrama (Figura 4.1). 


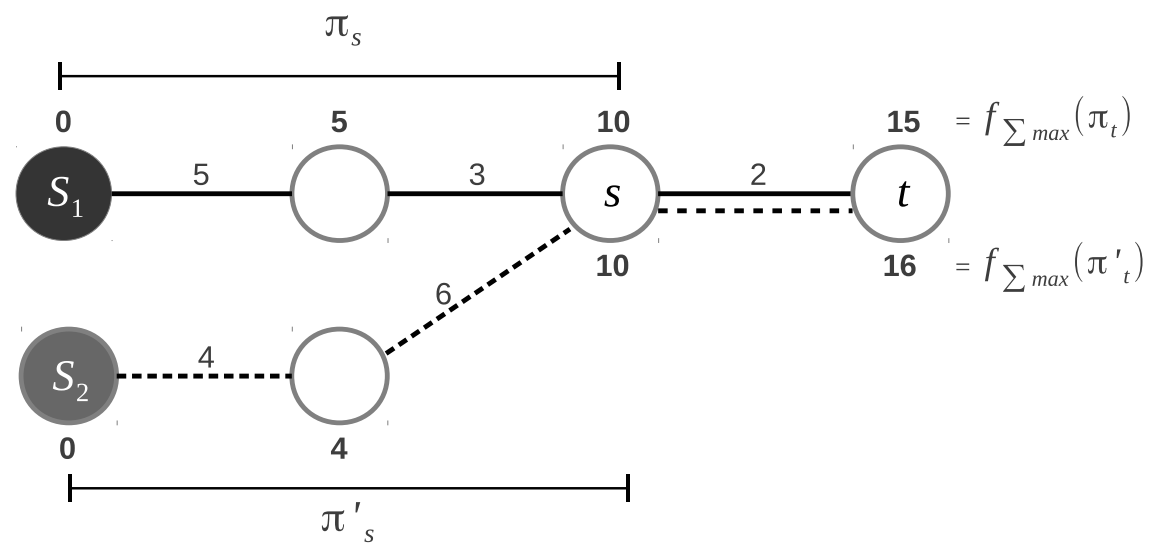

Figura A.2: Contra exemplo da condição $C 4$ para a função $f_{\Sigma \max }$, onde $\pi_{t}=\pi_{s} \cdot\langle s, t\rangle, \pi_{s}$, e $\pi_{s}^{\prime}$ são caminhos ótimos, $\pi_{t}^{\prime}=\pi_{s}^{\prime} \cdot\langle s, t\rangle$ é um caminho não ótimo, e $S_{1}, S_{2}$ são sementes.

\section{A.2 Função distância mínima de barreira $\left(f_{\uparrow}\right)$}

Seja a função $f_{\uparrow}$ definida por:

$$
\begin{aligned}
f_{\uparrow}\left(\pi_{t}=\langle t\rangle\right) & = \begin{cases}0, & \text { se } t \in \mathcal{S}, \\
+\infty, & \text { caso contrário. }\end{cases} \\
f_{\uparrow}\left(\pi_{t}=\pi_{s} \cdot\langle s, t\rangle\right) & =f_{\text {Imax }}\left(\pi_{t}\right)-f_{\text {Imin }}\left(\pi_{t}\right),
\end{aligned}
$$

onde $\mathcal{S}$ é um conjunto de sementes, e $f_{\text {Imax }}$ e $f_{\text {Imin }}$ são funções que tomam os valores máximo e mínimo da intensidade ao longo do caminho, respectivamente:

$$
\begin{aligned}
f_{\operatorname{Imax}}\left(\pi_{t}=\langle t\rangle\right) & =I(t) \\
f_{\operatorname{Imax}}\left(\pi_{t}=\pi_{s} \cdot\langle s, t\rangle\right) & =\max \left\{f_{\operatorname{Imax}}\left(\pi_{s}\right), I(t)\right\},
\end{aligned}
$$

e

$$
\begin{aligned}
f_{\text {Imin }}\left(\pi_{t}=\langle t\rangle\right) & =I(t) \\
f_{\text {Imin }}\left(\pi_{t}=\pi_{s} \cdot\langle s, t\rangle\right) & =\min \left\{f_{\text {Imin }}\left(\pi_{s}\right), I(t)\right\},
\end{aligned}
$$

onde, $I(t)$ representa a intensidade do pixel $t$.

A função $f_{\uparrow}$ é uma função não suave que pertence ao subconjunto $C_{1} \backslash\left(C_{2} \bigcup C_{4}\right)$ no diagrama de classificação de funções de conexidade (Figura 4.1), de modo que somente satisfaz a condição $C 1$ de suavidade (Subseção 3.1.3).

\section{Prova:}

C1. $f_{\uparrow}\left(\pi_{s}\right) \leq f_{\uparrow}\left(\pi_{t}=\pi_{s} \cdot\langle s, t\rangle\right)$.

Como

$$
\begin{aligned}
f_{\operatorname{Imax}}\left(\pi_{s}\right) & \leq f_{\operatorname{Imax}}\left(\pi_{t}\right) \mathrm{e} \\
f_{\operatorname{Imin}}\left(\pi_{t}\right) & \leq f_{\operatorname{Imin}}\left(\pi_{s}\right)
\end{aligned}
$$


então,

$$
\begin{aligned}
& f_{\operatorname{Imax}}\left(\pi_{s}\right)+f_{\operatorname{Imin}}\left(\pi_{t}\right) \leq f_{\operatorname{Imax}}\left(\pi_{t}\right)+f_{\operatorname{Imin}}\left(\pi_{s}\right), \quad \mathrm{e} \\
& f_{\operatorname{Imax}}\left(\pi_{s}\right)-f_{\operatorname{Imin}}\left(\pi_{s}\right) \leq f_{\operatorname{Imax}}\left(\pi_{t}\right)-f_{\operatorname{Imin}}\left(\pi_{t}\right) .
\end{aligned}
$$

Assim, da Equação A.2 nós obtemos $f_{\uparrow}\left(\pi_{s}\right) \leq f_{\uparrow}\left(\pi_{t}\right)$.

Portanto, $f_{\uparrow}$ satisfaz a condição $C 1$.

C2. $\pi_{s}$ é ótimo.

Na Figura A.3, para o caminho ótimo $\pi_{t}=\pi_{s} \cdot\langle s, t\rangle$, nós temos que $\pi_{s}$ é não ótimo, dado que existe outro caminho $\pi_{s}^{\prime}$ o qual oferece um melhor custo do que $\pi_{s}$ a $s$, isto é,

$$
f_{\uparrow}\left(\pi_{s}^{\prime}\right)=6 \leq f_{\uparrow}\left(\pi_{s}\right)=7 .
$$

Uma vez que $\pi_{t}^{\prime}$ não é ótimo, temos que a condição $C_{2}$ é violada por $f_{\uparrow}$.

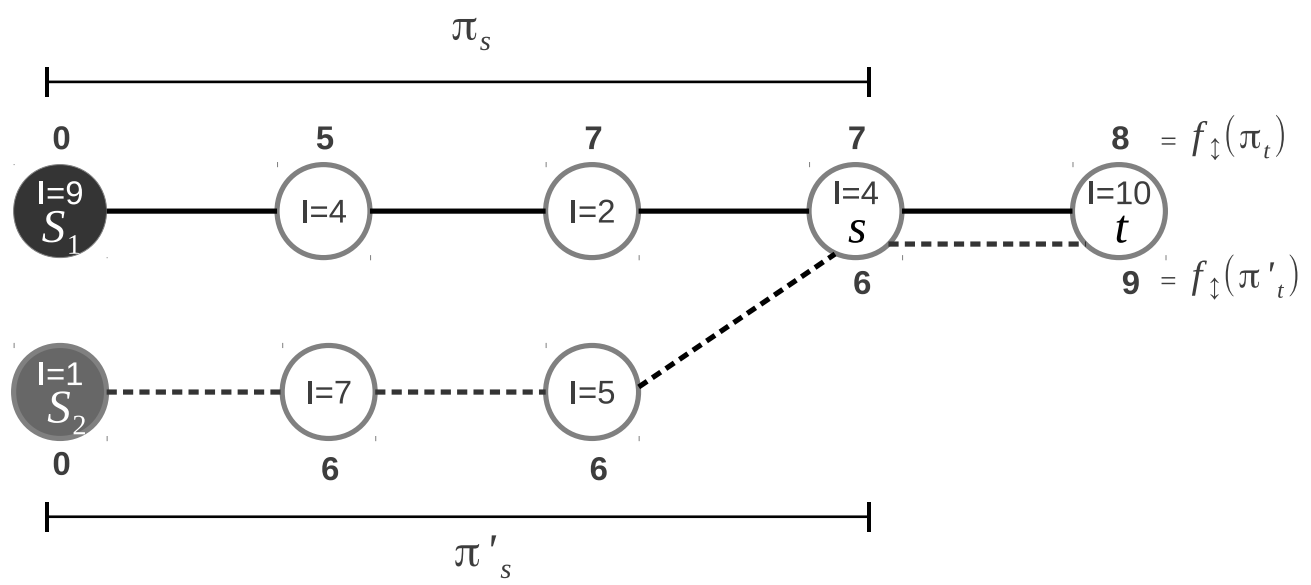

Figura A.3: Contra exemplo para a segunda e terceira condiçôes (C2 e C3) para a função $f_{\uparrow}$, onde $\pi_{t}=$ $\pi_{s} \cdot\langle s, t\rangle$ é um caminho ótimo, e $\pi_{t}^{\prime}=\pi_{s}^{\prime} \cdot\langle s, t\rangle$ é um caminho ótimo, a partir das sementes $S_{1}$ e $S_{2}$, respectivamente.

C3. Para qualquer caminho ótimo $\pi_{s}^{\prime}$ com destino em $s, f_{\uparrow}\left(\pi_{s}^{\prime} \cdot\langle s, t\rangle\right)=f_{\uparrow}\left(\pi_{t}\right)$.

Na Figura A.4 nós temos

$$
f_{\uparrow}\left(\pi_{s}^{\prime} \cdot\langle s, t\rangle\right)=7 \quad \neq \quad f_{\uparrow}\left(\pi_{t}=\pi_{s} \cdot\langle s, t\rangle\right)=5 .
$$

Assim, nós temos que $f_{\uparrow} \notin C_{3}$.

C4. Para quaisquer caminhos $\pi_{s}$ e $\pi_{s}^{\prime}$ com destino em $s$, se $f_{\uparrow}\left(\pi_{s}\right)=f_{\uparrow}\left(\pi_{s}^{\prime}\right)$, então $f_{\uparrow}\left(\pi_{s} \cdot\langle s, t\rangle\right)=$ $f_{\Uparrow}\left(\pi_{s}^{\prime} \cdot\langle s, t\rangle\right)$.

Na Figura A.4, para os caminhos ótimos $\pi_{s}$ e $\pi_{s}^{\prime}$ nós temos:

$$
f_{\uparrow}\left(\pi_{s} \cdot\langle s, t\rangle\right)=5 \neq f_{\uparrow}\left(\pi_{s}^{\prime} \cdot\langle s, t\rangle\right)=7 .
$$

Consequentemente, $f_{\uparrow}$ não satisfaz esta condição $\left(f_{\uparrow} \notin C_{4}\right)$. 


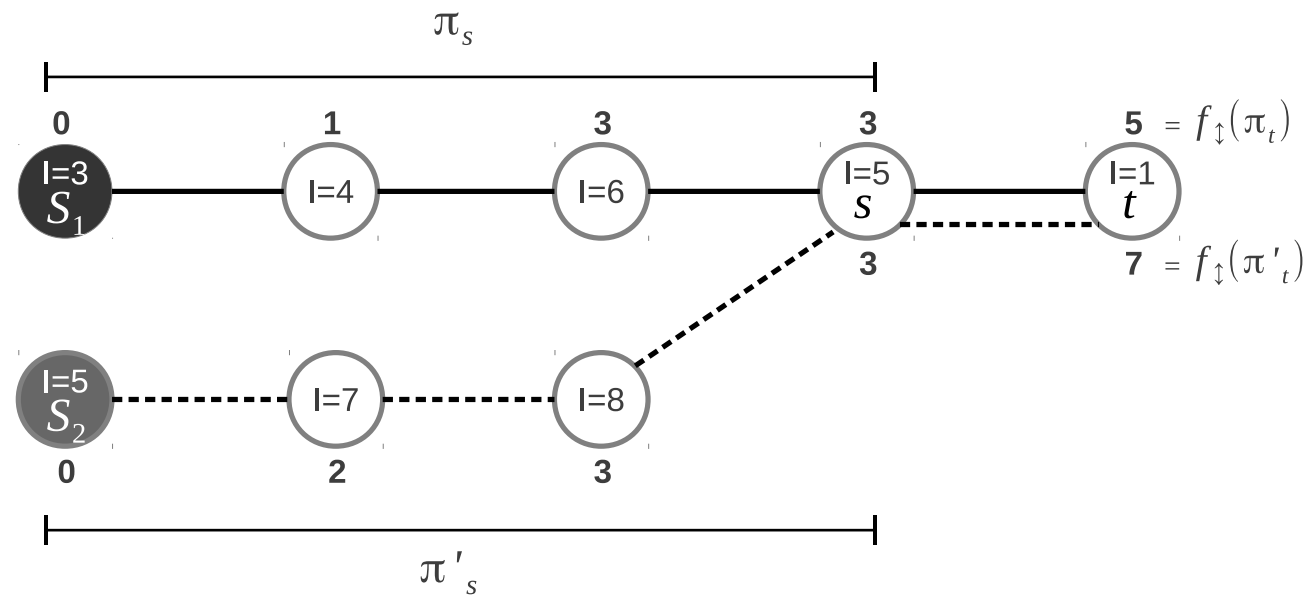

Figura A.4: Contra exemplo da condição $C 4$ para a função $f_{\uparrow}$, onde $\pi_{t}=\pi_{s} \cdot\langle s, t\rangle, \pi_{s}$, e $\pi_{s}^{\prime}$ são caminhos ótimos; $\pi_{t}^{\prime}=\pi_{s}^{\prime} \cdot\langle s, t\rangle$ é um caminho não ótimo, e $S_{1}, S_{2}$ são sementes.

Portanto, como a função $f_{\uparrow}$ somente satisfaz a condição $C 1$ de suavidade (Subseção 3.1.3), podemos concluir que $f_{\uparrow}$ é uma função não suave e $f_{\uparrow} \in C_{1} \backslash\left(C_{2} \bigcup C_{4}\right)$ no diagrama (Figura 4.1).

\section{A.3 Função amplitude máxima das intensidades relativas $\left(f_{\max |\triangle I|}\right)$}

Seja a função $f_{\max |\triangle I|}$ definida por:

$$
\begin{aligned}
f_{\max |\triangle I|}\left(\pi_{t}=\langle t\rangle\right) & = \begin{cases}0, & \text { se } t \in S, \\
+\infty, & \text { caso contrário. }\end{cases} \\
f_{\max |\triangle I|}\left(\pi_{t}=\pi_{s} \cdot\langle s, t\rangle\right) & =\max \left\{f_{\max |\triangle I|}\left(\pi_{s}\right),\left|I(t)-I\left(R\left(\pi_{s}\right)\right)\right|\right\},
\end{aligned}
$$

onde $R\left(\pi_{s}\right)$ é o pixel raiz (origem) do caminho $\pi_{s}$.

A função $f_{\max |\triangle I|}$ é uma função não suave que pertence ao subconjunto $C_{1} \backslash\left(C_{2} \bigcup C_{4}\right)$ no diagrama de classificação de funções de conexidade (Figura 4.1), de modo que somente satisfaz a condição $C 1$ de suavidade (Subseção 3.1.3).

\section{Prova:}

C1. $f_{\max |\triangle I|}\left(\pi_{s}\right) \leq f_{\max |\triangle I|}\left(\pi_{t}=\pi_{s} \cdot\langle s, t\rangle\right)$.

Suponhamos que essa condição é verdadeira, então da Equação A.5 nós temos:

$$
f_{\max |\triangle I|}\left(\pi_{s}\right) \leq \max \left\{f_{\max |\triangle I|}\left(\pi_{s}\right),\left|I(t)-I\left(R\left(\pi_{s}\right)\right)\right|\right\}
$$

que é claramente correto, devido à função máxima no segundo termo. Portanto, $f_{\max |\triangle I|}\left(\pi_{s}\right)$ satisfaz a condição $C 1\left(f_{\max |\triangle I|} \in C_{1}\right)$.

C2. $\pi_{s}$ é ótimo.

Na Figura A.5, para o caminho $\pi_{t}=\pi_{s} \cdot\langle s, t\rangle$, nós temos que $\pi_{s}$ não é ótimo, dado que existe 
outro caminho $\pi_{s}^{\prime}$ o qual oferece um melhor custo do que $\pi_{s}$ para $s$, isto é,

$$
f_{\max |\triangle I|}\left(\pi_{s}^{\prime}\right)=7 \leq f_{\uparrow}\left(\pi_{s}\right)=8 .
$$

Como $\pi_{t}^{\prime}$ não é ótimo, então $f_{\max |\triangle I|}$ viola a condição $C 2\left(f_{\max |\triangle I|} \notin C_{2}\right)$.

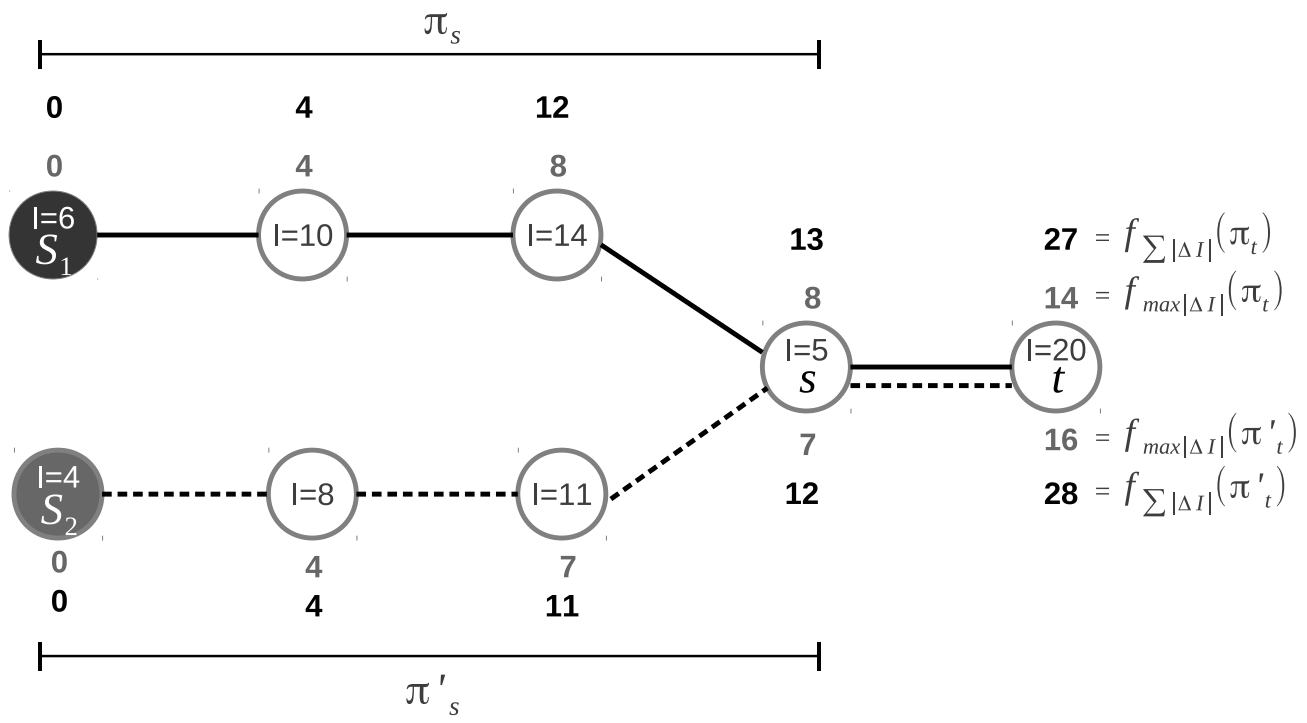

Figura A.5: Contra exemplo da segunda e terceira condições (C2 e C3) para as funções $f_{\max |\triangle I|}$ e $f_{\Sigma|\triangle I|}$. $O$ caminho $\pi_{t}=\pi_{s} \cdot\langle s, t\rangle$ é um caminho ótimo, e $\pi_{t}^{\prime}=\pi_{s}^{\prime} \cdot\langle s, t\rangle$ não é um caminho ótimo, a partir das sementes $S_{1}$ e $S_{2}$, respectivamente.

C3. Para qualquer caminho ótimo $\pi_{s}^{\prime}, f_{\max |\triangle I|}\left(\pi_{s}^{\prime} \cdot\langle s, t\rangle\right)=f_{\max |\triangle I|}\left(\pi_{t}\right)$.

Nós podemos ver um contra exemplo na Figura A.6, onde essa condição é violada, pois

$$
f_{\max |\triangle I|}\left(\pi_{s}^{\prime} \cdot\langle s, t\rangle\right)=16 \neq f_{\max |\triangle I|}\left(\pi_{t}\right)=14 .
$$

C4. Para quaisquer caminhos $\pi_{s}$ e $\pi_{s}^{\prime}$ com destino em $s$, se $f_{\max |\triangle I|}\left(\pi_{s}\right)=f_{\max |\triangle I|\left(\pi_{s}^{\prime}\right)}$, então $f_{\max |\triangle I|}\left(\pi_{s} \cdot\langle s, t\rangle\right)=f_{\max |\triangle I|}\left(\pi_{s}^{\prime} \cdot\langle s, t\rangle\right)$.

Na Figura A.6, nós podemos observar que:

$$
f_{\max |\triangle I|}\left(\pi_{s} \cdot\langle s, t\rangle\right)=14 \neq f_{\max |\triangle I|}\left(\pi_{s}^{\prime} \cdot\langle s, t\rangle\right)=16
$$

Assim, $f_{\max |\triangle I|}$ não satisfaz esta condição $\left(f_{\max |\triangle I|} \notin C_{4}\right)$.

Portanto, como a função $f_{\max |\triangle I|}$ somente satisfaz a condição $C 1$ de suavidade (Subseção 3.1.3), podemos concluir que $f_{\max |\triangle I|}$ é uma função não suave e $f_{\max |\triangle I|} \in C_{1} \backslash\left(C_{2} \bigcup C_{4}\right)$ no diagrama (Figura 4.1). 


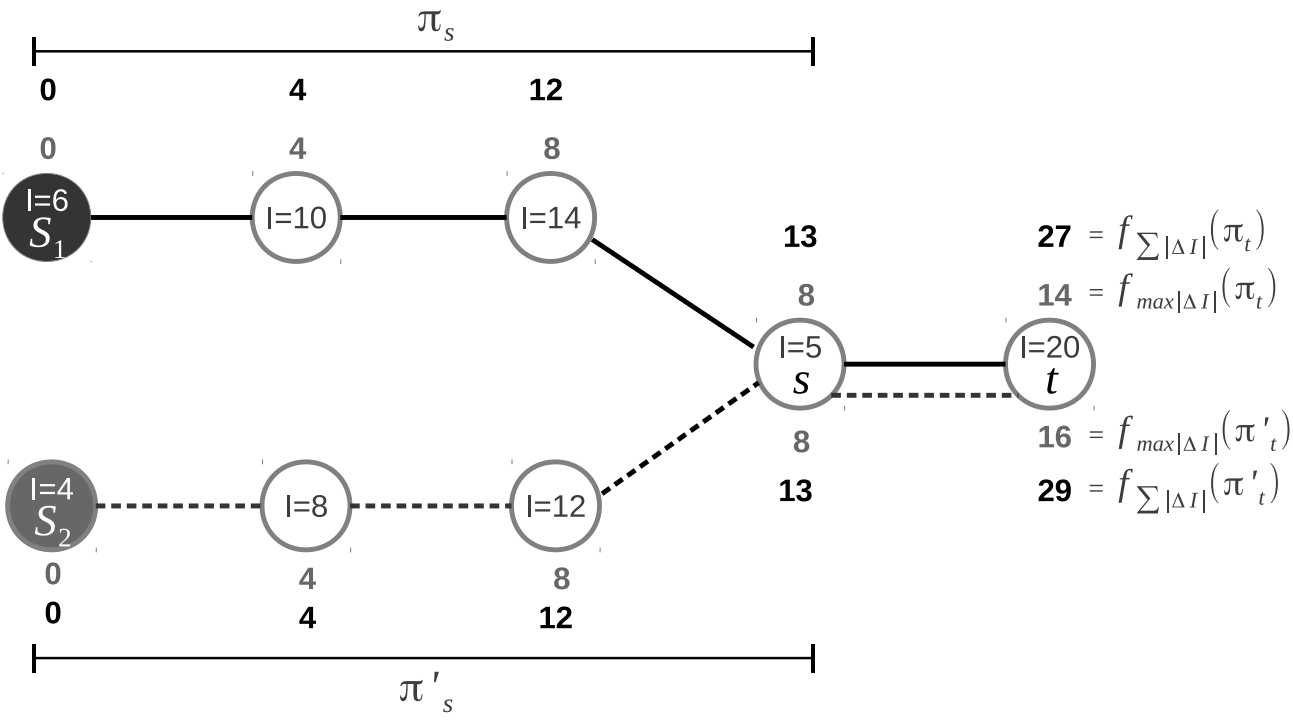

Figura A.6: Contra exemplo da condição C4 para as funções $f_{\max |\triangle I|}$ e $f_{\Sigma|\triangle I|}$. Os caminhos $\pi_{t}=\pi_{s} \cdot\langle s, t\rangle$, $\pi_{s}$, e $\pi_{s}^{\prime}$ são ótimos; $\pi_{t}^{\prime}=\pi_{s}^{\prime} \cdot\langle s, t\rangle$ é um caminho não ótimo, e $S_{1}$ e $S_{2}$ são sementes.

\section{A.4 Função soma do valor absoluto das intensidades relativas $\left(f_{\sum|\triangle I|}\right)$}

Seja a função $f_{\sum|\triangle I|}$ definida por:

$$
\begin{aligned}
f_{\sum|\triangle I|}\left(\pi_{t}=\langle t\rangle\right) & = \begin{cases}0, & \text { se } t \in S, \\
+\infty, & \text { caso contrário. }\end{cases} \\
f_{\sum|\triangle I|}\left(\pi_{t}=\pi_{s} \cdot\langle s, t\rangle\right) & =f_{\sum|\triangle I|}\left(\pi_{s}\right)+\left|I(t)-I\left(R\left(\pi_{s}\right)\right)\right|,
\end{aligned}
$$

onde $R\left(\pi_{s}\right)$ é o pixel raiz (origem) do caminho $\pi_{s}$.

A função $f_{\sum|\triangle I|}$ é uma função não suave que pertence ao subconjunto $C_{1} \backslash\left(C_{2} \bigcup C_{4}\right)$ no diagrama de classificação de funções de conexidade (Figura 4.1), de modo que somente satisfaz a condição $C 1$ de suavidade (Subseção 3.1.3).

\section{Prova:}

C1. $f_{\Sigma|\triangle I|}\left(\pi_{s}\right) \leq f_{\Sigma|\triangle I|}\left(\pi_{t}=\pi_{s} \cdot\langle s, t\rangle\right)$.

Suponhamos que a condição é verdadeira, então da Equação A.6 nós temos:

$$
f_{\Sigma|\triangle I|}\left(\pi_{s}\right) \leq f_{\sum|\triangle I|}\left(\pi_{s}\right)+\left|I(t)-I\left(R\left(\pi_{s}\right)\right)\right| .
$$

Como $\left|I(t)-I\left(R\left(\pi_{s}\right)\right)\right| \geq 0$ essa relação é correta, assim a condição $C 1$ é satisfeita $\left(f_{\Sigma|\triangle I|} \in\right.$ $\left.C_{1}\right)$.

C2. $\pi_{s}$ é ótimo.

Na Figura A.5, para um caminho ótimo $\pi_{t}=\pi_{s} \cdot\langle s, t\rangle$, nós temos que $\pi_{s}$ não é ótimo, dado 
que existe um outro caminho $\pi_{s}^{\prime}$ que tem um melhor custo do que $\pi_{s}$, isto é,

$$
f_{\Sigma|\triangle I|}\left(\pi_{s}^{\prime}\right)=12 \leq f_{\uparrow}\left(\pi_{s}\right)=13
$$

Assim, essa condição não é satisfeita $\left(f_{\Sigma|\triangle I|} \notin C_{2}\right)$.

C3. Para qualquer caminho ótimo $\pi_{s}^{\prime}, f_{\Sigma|\triangle I|}\left(\pi_{s}^{\prime} \cdot\langle s, t\rangle\right)=f_{\Sigma|\triangle I|}\left(\pi_{t}\right)$.

O contra exemplo na Figura A.6 mostra que essa condição é violada, pois

$$
f_{\Sigma|\triangle I|}\left(\pi_{s}^{\prime} \cdot\langle s, t\rangle\right)=29 \neq f_{\Sigma|\triangle I|}\left(\pi_{t}\right)=27
$$

C4. Para quaisquer caminhos $\pi_{s}$ e $\pi_{s}^{\prime}$ com destino em $s$, se $f_{\Sigma|\triangle I|}\left(\pi_{s}\right)=f_{\Sigma|\triangle I|}\left(\pi_{s}^{\prime}\right)$, então $f_{\Sigma|\triangle I|}\left(\pi_{s}\right.$. $\langle s, t\rangle)=f_{\Sigma|\triangle I|}\left(\pi_{s}^{\prime} \cdot\langle s, t\rangle\right)$.

Na Figura A.6, para caminhos ótimos $\pi_{s}$ e $\pi_{s}^{\prime}$ nós temos:

$$
f_{\Sigma|\triangle I|}\left(\pi_{s} \cdot\langle s, t\rangle\right)=27 \quad \neq \quad f_{\Sigma|\triangle I|}\left(\pi_{s}^{\prime} \cdot\langle s, t\rangle\right)=29
$$

Assim, $f_{\Sigma|\triangle I|}$ não satisfaz a condição $C 4\left(f_{\Sigma|\triangle I|} \notin C_{4}\right)$.

Portanto, como a função $f_{\Sigma|\triangle I|}$ somente satisfaz a condição $C 1$ de suavidade (Subseção 3.1.3), podemos concluir que $f_{\Sigma|\triangle I|}$ é uma função não suave e $f_{\Sigma|\triangle I|} \in C_{1} \backslash\left(C_{2} \cup C_{4}\right)$ no diagrama (Figura 4.1).

\section{A.5 Função de intensidade $\left(f_{I}\right)$}

Seja a função $f_{I}$ definida por:

$$
\begin{aligned}
f_{I}\left(\pi_{t}=\langle t\rangle\right) & = \begin{cases}0, & \text { se } t \in S, \\
+\infty, & \text { caso contrário. }\end{cases} \\
f_{I}\left(\pi_{t}=\pi_{s} \cdot\langle s, t\rangle\right) & =I(t),
\end{aligned}
$$

onde $S$ é o conjunto de sementes.

A função $f_{I}$ é uma função não suave que pertence ao subconjunto $\left(C_{2} \bigcap C_{4}\right) \backslash C_{1}$ no diagrama de classificação de funções de conexidade (Figura 4.1), de modo que somente viola a condição $C 1$ de suavidade (Subseção 3.1.3).

\section{Prova:}

C1. $f_{I}\left(\pi_{s}\right) \leq f_{I}\left(\pi_{t}=\pi_{s} \cdot\langle s, t\rangle\right)$.

Na Figura A.7 nós temos

$$
f_{I}\left(\pi_{s}\right)=30 \quad \not \leq \quad f_{I}\left(\pi_{t}\right)=20 .
$$

Assim, esta condição é violada. 


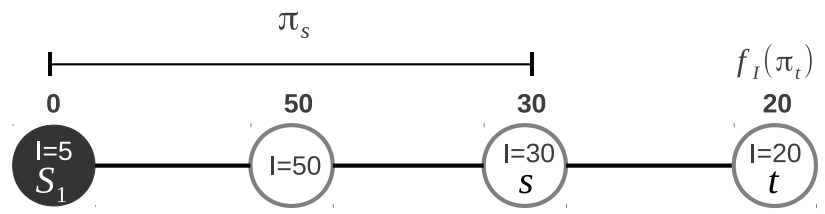

Figura A.7: Contra exemplo da primeira condição (C1) para a função $f_{I}$, onde $\pi_{t}=\pi_{s} \cdot\langle s, t\rangle$ é um caminho ótimo a partir da semente $S_{1}$.

C2. $\pi_{s}$ é ótimo.

Suponhamos que dado um caminho ótimo $\pi_{t}=\pi_{s} \cdot\langle s, t\rangle$, o caminho $\pi_{s}$ não é ótimo, então nós teríamos que existe um caminho $\pi_{s}^{\prime}$ tal que

$$
f_{I}\left(\pi_{s}^{\prime}\right)=I(s)<f_{I}\left(\pi_{s}\right)=I(s)
$$

o que é falso, dado que $I(s) \nless I(s)$. Portanto, a condição $C 2$ é satisfeita por $f_{I}$.

C3. Para qualquer caminho ótimo $\pi_{s}^{\prime}$ com destino em $s, f_{I}\left(\pi_{s}^{\prime} \cdot\langle s, t\rangle\right)=f_{I}\left(\pi_{t}\right)$.

Esta condição é satisfeita, uma vez que $f_{I}\left(\pi_{s}^{\prime} \cdot\langle s, t\rangle\right)=I(t)$ e $f_{I}\left(\pi_{t}\right)=I(t)$.

C4. Para quaisquer caminhos $\pi_{s}$ e $\pi_{s}^{\prime}$ com destino em $s$, se $f_{I}\left(\pi_{s}\right)=f_{I}\left(\pi_{s}^{\prime}\right)$, então $f_{I}\left(\pi_{s} \cdot\langle s, t\rangle\right)=$ $f_{I}\left(\pi_{s}^{\prime} \cdot\langle s, t\rangle\right)$.

Esta condição é satisfeita, uma vez que

$$
f_{I}\left(\pi_{s} \cdot\langle s, t\rangle\right)=I(t) \quad \text { e } \quad f_{I}\left(\pi_{s}^{\prime} \cdot\langle s, t\rangle\right)=I(t)
$$

Portanto, como a função $f_{I}$ somente viola a condição $C 1$ de suavidade (Subseção 3.1.3), podemos concluir que $f_{I}$ é uma função não suave e $f_{I} \in\left(C_{2} \bigcap C_{4}\right) \backslash C_{1}$ no diagrama (Figura 4.1).

\section{A.6 Função lexicográfica da soma das arestas máximas $\left(f_{\Sigma \max }^{l e x}\right)$}

Seja a função $f_{\Sigma \max }^{l e x}$ definida por:

$$
\begin{aligned}
f_{\Sigma \max }^{\text {lex }}\left(\pi_{t}=\langle t\rangle\right) & = \begin{cases}(0,0), & \text { se } t \in \mathcal{S}, \\
(+\infty,+\infty), & \text { caso contrário. }\end{cases} \\
f_{\Sigma \max }^{l e x}\left(\pi_{t}=\pi_{s} \cdot\langle s, t\rangle\right) & =\left(f_{\Sigma \max }\left(\pi_{t}\right), f_{\max }\left(\pi_{t}\right)\right) .
\end{aligned}
$$

onde $\mathcal{S}$ é um conjunto de sementes.

A função $f_{\Sigma \max }^{l e x}$ forma pares de valores que devem ser comparados de acordo com a ordem lexicográfica. A primeira componente é a função não suave $f_{\Sigma \max }$, e a segunda é a função suave $f_{\text {max }}$.

Do diagrama de classificação de funções de conexidade (Figura 4.1), temos que a função $f_{\Sigma \max }^{\text {lex }}$ é uma função não suave dado que $f_{\Sigma \max }^{\text {lex }} \in\left(C_{1} \cap C_{4}\right) \backslash C_{2}$; para avaliar isso vamos provar que condições satisfaz. 


\section{Prova:}

C1. $f_{\Sigma \max }^{\text {lex }}\left(\pi_{s}\right) \leq f_{\Sigma \max }^{\text {lex }}\left(\pi_{t}=\pi_{s} \cdot\langle s, t\rangle\right)$.

Suponhamos que esta condição é verdadeira. Então, da Equação A.9 nós temos:

$$
\left(f_{\Sigma \max }\left(\pi_{s}\right), f_{\max }\left(\pi_{s}\right)\right) \leq\left(f_{\Sigma \max }\left(\pi_{t}\right), f_{\max }\left(\pi_{t}\right)\right)
$$

Comparando os pares ordenados nós obtemos:

$$
f_{\Sigma \max }\left(\pi_{s}\right)<f_{\Sigma \max }\left(\pi_{t}\right)
$$

$\mathrm{Ou}$

$$
f_{\max }\left(\pi_{s}\right)=f_{\max }\left(\pi_{t}\right) \text { e } \quad f_{\max }\left(\pi_{s}\right) \leq f_{\max }\left(\pi_{t}\right)
$$

Dado que ambos $f_{\Sigma \max }$ e $f_{\max }$ satisfazem a condição $C 1$ para $\omega(s, t) \geq 0$ (Seção A.1), nós podemos concluir que $f_{\Sigma \max }^{\text {lex }} \in C_{1}$ para $\omega(s, t) \geq 0$.

C2. $\pi_{s}$ é ótimo.

Na Figura A.8, para o caminho ótimo $\pi_{t}=\pi_{s} \cdot\langle s, t\rangle$, nós temos que $\pi_{s}$ é não ótimo, dado que existe um outro caminho $\pi_{s}^{\prime}$ que oferece um melhor custo do que $\pi_{s}$, isto é,

$$
f_{\Sigma \max }^{l e x}\left(\pi_{s}^{\prime}\right)=(9,7) \leq f_{\Sigma \max }^{l e x}\left(\pi_{s}\right)=(10,5)
$$

Portanto, esta condição não é satisfeita por $f_{\Sigma \max }^{l e x}$.

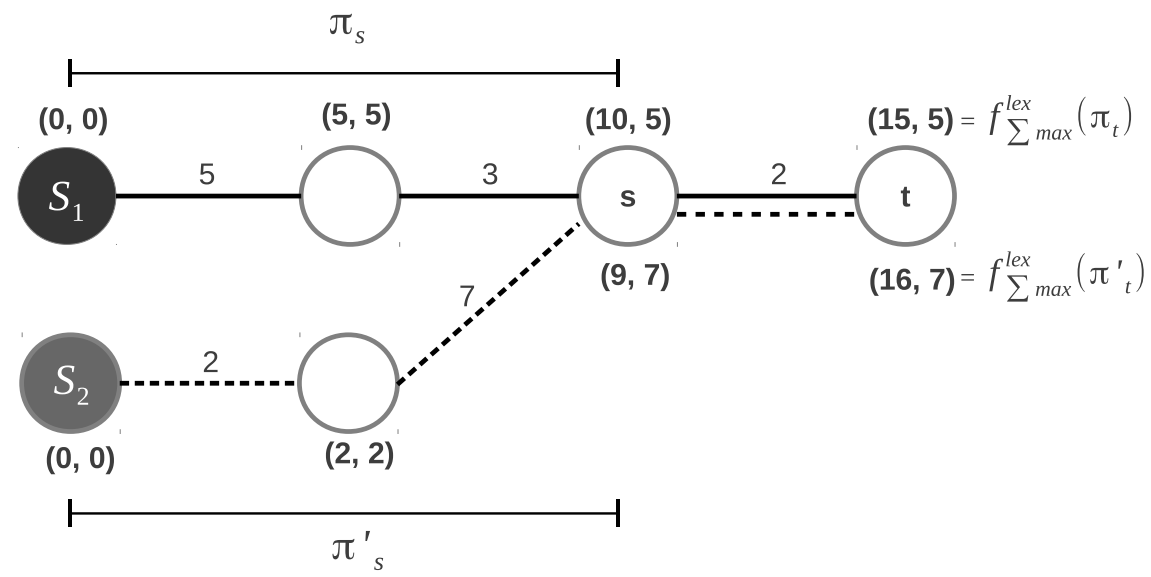

Figura A.8: Contra exemplo da segunda e terceira condiçôes (C2 e C3) para a função $f_{\Sigma \max }^{l e x}$, onde $\pi_{t}=$ $\pi_{s} \cdot\langle s, t\rangle$ é um caminho ótimo, e $\pi_{t}^{\prime}=\pi_{s}^{\prime} \cdot\langle s, t\rangle$ é um outro caminho não ótimo, a partir das sementes $S_{1} e$ $S_{2}$ respectivamente.

C3. Para qualquer caminho ótimo $\pi_{s}^{\prime}, f_{\Sigma \max }^{\text {lex }}\left(\pi_{s}^{\prime} \cdot\langle s, t\rangle\right)=f_{\Sigma \max }^{l e x}\left(\pi_{t}\right)$

Na Figura A.8 nós temos

$$
f_{\Sigma \max }^{l e x}\left(\pi_{s}^{\prime} \cdot\langle s, t\rangle\right)=(16,7) \neq f_{\Sigma \max }^{l e x}\left(\pi_{t}\right)=(15,5) .
$$

Portanto, esta condição é violada por $f_{\Sigma \max }^{l e x}$. 
C4. Para quaisquer caminhos $\pi_{s}$ e $\pi_{s}^{\prime}$ com destino em $s$, se $f_{\Sigma \max }^{\text {lex }}\left(\pi_{s}\right)=f_{\Sigma \max }^{\text {lex }}\left(\pi_{s}^{\prime}\right)$, então $f_{\Sigma \max }^{l e x}\left(\pi_{s} \cdot\langle s, t\rangle\right)=f_{\Sigma \max }^{l e x}\left(\pi_{s}^{\prime} \cdot\langle s, t\rangle\right)$.

Dado que $f_{\Sigma \max }^{l e x}\left(\pi_{s}\right)=f_{\Sigma \max }^{l e x}\left(\pi_{s}^{\prime}\right)$, da Equação A.9 nós obtemos

$$
\left(f_{\Sigma \max }\left(\pi_{s}\right), f_{\max }\left(\pi_{s}\right)\right)=\left(f_{\Sigma \max }\left(\pi_{s}^{\prime}\right), f_{\max }\left(\pi_{s}^{\prime}\right)\right)
$$

o qual implica que:

$$
\begin{aligned}
f_{\Sigma \max }\left(\pi_{s}\right) & =f_{\Sigma \max }\left(\pi_{s}^{\prime}\right), \mathrm{e} \\
f_{\max }\left(\pi_{s}\right) & =f_{\max }\left(\pi_{s}^{\prime}\right) .
\end{aligned}
$$

Portanto, pela definição de $f_{\max }$ e da Equação A.14

$$
\begin{aligned}
& f_{\max }\left(\pi_{s} \cdot\langle s, t\rangle\right)=\max \left\{f_{\max }\left(\pi_{s}^{\prime}\right), \omega(s, t)\right\} \\
& f_{\max }\left(\pi_{s} \cdot\langle s, t\rangle\right)=f_{\max }\left(\pi_{s}^{\prime} \cdot\langle s, t\rangle\right)
\end{aligned}
$$

e das Equações A.1, A.13 e A.15 nós temos

$$
\begin{aligned}
& f_{\Sigma \max }\left(\pi_{s} \cdot\langle s, t\rangle\right)=f_{\Sigma \max }\left(\pi_{s}^{\prime}\right)+f_{\max }\left(\pi_{s}^{\prime} \cdot\langle s, t\rangle\right), \\
& f_{\Sigma \max }\left(\pi_{s} \cdot\langle s, t\rangle\right)=f_{\Sigma \max }\left(\pi_{s}^{\prime} \cdot\langle s, t\rangle\right)
\end{aligned}
$$

Assim, das Equações A.15 e A.16 nós temos

$$
\begin{aligned}
& f_{\Sigma \max }^{l e x}\left(\pi_{s} \cdot\langle s, t\rangle\right)=\left(f_{\Sigma \max }\left(\pi_{s}^{\prime} \cdot\langle s, t\rangle\right), f_{\max }\left(\pi_{s}^{\prime} \cdot\langle s, t\rangle\right)\right), \\
& f_{\Sigma \max }^{\text {lex }}\left(\pi_{s} \cdot\langle s, t\rangle\right)=f_{\Sigma \max }^{\text {lex }}\left(\pi_{s}^{\prime} \cdot\langle s, t\rangle\right)
\end{aligned}
$$

Portanto, a condição $C 4$ é satisfeita por $f_{\Sigma \max }^{l e x}$.

Por conseguinte, nós temos que a função $f_{\Sigma \max }^{l e x}$ é uma função não suave que viola somente as condições $C 2$ e $C 3\left(f_{\Sigma \max }^{\text {lex }} \in\left(C_{1} \cap C_{4}\right) \backslash C_{2}\right)$. Note que $f_{\Sigma \max }^{\text {lex }}$ não viola a condição $C 4$, ao contrário de $f_{\Sigma \max }$. 
APÊNDICE A 


\section{Apêndice B}

\section{Prova das proposições para qualquer função $f \in\left(C_{1} \cap C_{4}\right) \backslash C_{2}$}

Neste apêndice apresentamos as provas das proposições propostas na Seção 5.1, dadas para funções de conexidade que satisfazem as condições $C 1$ (Subseção 3.1.3) e $C 4$ (Seção 4.1), mas não a condição $C 2$ (Subseção 3.1.3), ou seja funções pertencentes ao subconjunto $\left(C_{1} \bigcap C_{4}\right) \backslash C_{2}$ no diagrama de classificação de funções de conexidade (Figura 4.1).

\section{Proposição 1}

Considere uma função $f \in\left(C_{1} \bigcap C_{4}\right) \backslash C_{2}$. Para um grafo $G=(\mathcal{I}, \xi)$ da imagem, e conjunto $\mathcal{S}$ de sementes, dados, seja $\mathcal{O}$ o conjunto de todos os pixels $t \in \mathcal{I}$, tal que existe um caminho ótimo completo $\pi_{t}$ para $f$. Em qualquer floresta de espalhamento $P$ calculada em $G$ pelo algoritmo da IFT para $f$, todos os caminhos $\tau_{t}^{P} \operatorname{com} t \in \mathcal{O}$ são caminhos ótimos.

\section{Prova:}

Seja $\pi_{t}=\left\langle t_{1}, t_{2}, \ldots, t_{i}, \ldots, t_{n}=t\right\rangle$ um caminho ótimo completo dado para $f$, e $\tau_{t}^{P}=$ $\left\langle s_{1}, s_{2}, \ldots, s_{i}, \ldots, s_{m}=t\right\rangle$ o caminho calculado na floresta de espalhamento $P$. Nós temos a seguinte prova por indução matemática:

1. Base da indução: Mostrar que o enunciado vale para $n=1$.

Nesse caso, nós temos $t=t_{1}$, e portanto $\pi_{t}=\left\langle t_{1}\right\rangle$. Como $f\left(\left\langle t_{1}\right\rangle\right)$ é um valor finito, $t_{1}$ é inserido na fila $Q$ de prioridade (Linha 6 do Algoritmo 1 ). Dado que $f\left(\left\langle t_{1}\right\rangle\right)$ é ótimo, nós podemos afirmar com segurança que $t_{1}$ não sairá de $Q$ (Linha 8 do Algoritmo 1) com um custo $V\left(t_{1}\right)$ pior do que $f\left(\left\langle t_{1}\right\rangle\right)$, portanto, o caminho induzido $\tau_{t}^{P}$ é ótimo.

2. Passo da indução: Suponhamos que o enunciado é verdadeiro para o caminho ótimo completo $\pi_{t_{i}}$, com $i \geq 1$. Nós devemos provar que isso também vale para o caminho ótimo completo $\pi_{t_{i+1}}=\pi_{t_{i}} \cdot\left\langle t_{i}, t_{i+1}\right\rangle$.

Pela hipótese nós temos que o caminho $\tau_{t_{i}}^{P}$ em $P$ é ótimo. Dado que a condição $C 4$ é satisfeita $\left(f \in\left(C_{1} \cap C_{4}\right) \backslash C_{2}\right)$, nós podemos concluir que $f\left(\tau_{t_{i}}^{P} \cdot\left\langle t_{i}, t_{i+1}\right\rangle\right)=f\left(\pi_{t_{i}} \cdot\left\langle t_{i}, t_{i+1}\right\rangle\right)=f\left(\pi_{t_{i+1}}\right)$, assim, podemos concluir que $\tau_{t_{i}}^{P} \cdot\left\langle t_{i}, t_{i+1}\right\rangle$ é ótimo. 
Pela construção do algoritmo, se $t_{i+1}$ ainda não foi conquistado, nós temos que esse caminho estendido $\tau_{t_{i}}^{P} \cdot\left\langle t_{i}, t_{i+1}\right\rangle$ será avaliado pelo algoritmo da IFT (Linhas 10 a 14 do Algoritmo 1), e uma vez que ele é ótimo, ele não será substituído posteriormente por qualquer outro caminho (como garantido pela desigualdade estrita na Linha 12 do Algoritmo 1). Se $t_{i+1}$ já foi conquistado por um caminho diferente em $P$, o seu custo não pode ser pior do que $f\left(\pi_{t_{i+1}}\right)$, uma vez que $f \in C_{1}$. Portanto, no final, $\tau_{t_{i+1}}^{P}$ será um caminho ótimo.

\section{Proposição 2}

Considere uma função de conexidade $f$, classificada como $f \in\left(C_{1} \cap C_{4}\right) \backslash C_{2}$ para um grafo geral. Se para um grafo particular $G=(\mathcal{I}, \xi)$ da imagem e conjunto $\mathcal{S}$ de sementes, dados, existe uma floresta de caminhos ótimos para $f$, então qualquer floresta de espalhamento $P$ calculada em $G$ pelo algoritmo da IFT (Algoritmo 1) para $f$ é uma floresta de caminhos ótimos.

\section{Prova:}

Essa proposição é uma consequência imediata da Proposição 1, pois qualquer caminho induzido por uma floresta de caminhos ótimos é um caminho ótimo completo (Subseção 3.1.4), portanto se existe uma floresta de caminhos ótimos para $f$, então $\mathcal{O}=\mathcal{I}$ na Proposição 1. 


\section{Apêndice C}

\section{Prova dos teoremas de otimalidade dos cortes internos/externos na OIFT}

Neste apêndice apresentamos as provas dos Teoremas 1 e 2 da Seção 6.2.

\section{Teorema 1 (Otimalidade dos cortes internos/externos)}

Para um grafo $G=(\mathcal{I}, \xi)$ da imagem, e dois conjuntos $\mathcal{S}_{o}$ e $\mathcal{S}_{b}$ de sementes, se existe uma floresta de caminhos ótimos para a função $f_{o, \max }^{b k g}$, então seu corte é um corte ótimo que maximiza $E_{o}(\hat{L})$ entre todos os possíveis resultados da segmentação satisfazendo as restrições fortes. Se existe uma floresta de caminhos ótimos para a função $f_{i \text {,max }}^{b k g}$, então seu corte é um corte ótimo que maximiza $E_{i}(\hat{L})$ entre todos os possíveis resultados da segmentação satisfazendo as restrições fortes.

\section{Prova:}

Nós provaremos o Teorema 1 para o caso da função $f_{o, \max }^{b k g}$ (Equação 6.5), pois a função $f_{i, \max }^{b k g}$ (Equação 6.4) tem essencialmente uma prova idêntica.

Em uma floresta de caminhos ótimos para a função $f_{o, \max }^{b k g}$, para qualquer aresta $(a, b)$ tal que $a$ pertence ao objeto $(L(a)=1)$ e $b$ ao fundo $(L(b)=0)$, a seguinte desigualdade é verdadeira:

$$
f_{o, \max }^{b k g}\left(\pi_{b} \cdot\langle b, a\rangle\right)>f_{o, \max }^{b k g}\left(\pi_{a}\right)
$$

Essa desigualdade é uma consequência da otimalidade do caminho (Equação 3.6) para a função $f_{o, \max }^{b k g}$. Por exemplo, se a extensão do caminho $\pi_{b}$ pela aresta $(b, a)$ tem um custo menor do que o caminho $\pi_{a}$, ou seja $f_{o, \max }^{b k g}\left(\pi_{b} \cdot\langle b, a\rangle\right)<f_{o, \max }^{b k g}\left(\pi_{a}\right)$, então esse caminho $\pi_{a}$ não é ótimo. O caso no qual $f_{o, \max }^{b k g}\left(\pi_{b} \cdot\langle b, a\rangle\right)=f_{o, \max }^{b k g}\left(\pi_{a}\right)$ não é possível, pois custos de caminhos com rótulos diferentes são números pares ou ímpares. Portanto, a Equação C.1 é a única configuração válida possível.

$\mathrm{Na}$ verdade, podemos concluir também as seguintes equações:

$$
\begin{array}{r}
2 \times \omega(a, b)+1>f_{o, \max }^{b k g}\left(\pi_{a}\right) \\
2 \times \omega(a, b) \geq f_{o, \max }^{b k g}\left(\pi_{b}\right)
\end{array}
$$


A primeira desigualdade (Equação C.2) pode ser provada por contradição como segue:

- Se $2 \times \omega(a, b)+1 \leq f_{o, \max }^{b k g}\left(\pi_{a}\right)$, então

$$
\begin{aligned}
& f_{o, \text { max }}^{b k g}\left(\pi_{a} \cdot\langle a, b\rangle\right)=\max \left\{f_{o, \text { max }}^{b k g}\left(\pi_{a}\right), 2 \times \omega(a, b)+1\right\}, \\
& f_{o, \text { max }}^{b k g}\left(\pi_{a} \cdot\langle a, b\rangle\right)=f_{o, \text { max }}^{b k g}\left(\pi_{a}\right) .
\end{aligned}
$$

Existem duas possibilidades, a extensão do caminho $\pi_{a}$ pela aresta $(a, b)$ tem valor

$$
f_{o, \max }^{b k g}\left(\pi_{a}\right)<f_{o, \max }^{b k g}\left(\pi_{b}\right)
$$

$\mathrm{Ou}$

$$
f_{o, \max }^{b k g}\left(\pi_{a}\right) \geq f_{o, \max }^{b k g}\left(\pi_{b}\right)
$$

Se o primeiro caso (Equação C.4) é verdadeiro, então $\pi_{b}$ não é ótimo levando a uma contradição.

No segundo caso (Equação C.5), temos que

$$
f_{o, \max }^{b k g}\left(\pi_{b} \cdot\langle b, a\rangle\right)=\max \left\{f_{o, \max }^{b k g}\left(\pi_{b}\right), 2 \times \omega(a, b)\right\} \leq f_{o, \max }^{b k g}\left(\pi_{a}\right),
$$

uma vez que $2 \times \omega(a, b)+1 \leq f_{o, \max }^{b k g}\left(\pi_{a}\right)$ e $f_{o, \max }^{b k g}\left(\pi_{a}\right) \geq f_{o, \max }^{b k g}\left(\pi_{b}\right)$, mas isso é inválido de acordo com a Equação C.1. Portanto, $2 \times \omega(a, b)+1>f_{o, \max }^{b k g}\left(\pi_{a}\right)$ (Equação C.2) é a única configuração válida.

A segunda desigualdade (Equação C.3) também pode ser provada por contradição.

- Se $2 \times \omega(a, b)<f_{o, \max }^{b k g}\left(\pi_{b}\right)$, então

$$
f_{o, \max }^{b k g}\left(\pi_{b} \cdot\langle b, a\rangle\right)=\max \left\{f_{o, \max }^{b k g}\left(\pi_{b}\right), 2 \times \omega(a, b)\right\}=f_{o, \max }^{b k g}\left(\pi_{b}\right),
$$

mas dado que $a$ pertence ao objeto, segundo Equação C.1 temos que

$$
f_{o, \max }^{b k g}\left(\pi_{a}\right)<f_{o, \max }^{b k g}\left(\pi_{b}\right) .
$$

Mas isso implica por sua vez que

$$
f_{o, \max }^{b k g}\left(\pi_{a} \cdot\langle a, b\rangle\right)=\max \left\{f_{o, \max }^{b k g}\left(\pi_{a}\right), 2 \times \omega(a, b)+1\right\} \leq f_{o, \max }^{b k g}\left(\pi_{b}\right) .
$$

O caso $f_{o, \max }^{b k g}\left(\pi_{a} \cdot\langle a, b\rangle\right)=f_{o, \max }^{b k g}\left(\pi_{b}\right)$ não é possível por construção (custos a partir de diferentes rótulos são números pares ou ímpares). Assim, $f_{o, \max }^{b k g}\left(\pi_{a} \cdot\langle a, b\rangle\right)<f_{o, \max }^{b k g}\left(\pi_{b}\right)$ é a única configuração possível. Mas isso leva a uma contradição, pois nesse caso $b$ poderia não pertencer ao fundo, provando a desigualdade dada na Equação C.3. 
Então, agora vamos considerar dois segmentos candidatos arbitrários de borda orientada, como dois conjuntos $\mathcal{X}_{1}$ e $\mathcal{X}_{2}$ de arestas apontando para o exterior do objeto candidato que esses segmentos representam. Sejam as arestas $\left(u_{1}, v_{1}\right) \in \mathcal{X}_{1}$ e $\left(u_{2}, v_{2}\right) \in \mathcal{X}_{2}$ arestas com peso mínimo dentro desses conjuntos. Esses conjuntos incluem ambos cenários representados na Figura C.1 (a borda inteira, ou apenas parte dela).

De acordo com o critério de otimização da energia (Seção 4.4) qualquer parte de uma borda de corte é escolhida como aquela que maximiza seu peso mínimo (por exemplo, Figura 4.3), assim nós temos que um conjunto $\mathcal{X}_{\mathrm{i}}$ com maior peso mínimo apontando para o exterior deve ser selecionado. Existem dois casos, $\omega\left(u_{1}, v_{1}\right)>\omega\left(u_{2}, v_{2}\right)$ ou $\omega\left(u_{1}, v_{1}\right)<\omega\left(u_{2}, v_{2}\right)$.

No primeiro caso, temos que $\omega\left(u_{1}, v_{1}\right)>\omega\left(u_{2}, v_{2}\right)$ e de acordo com o Teorema $1, \mathcal{X}_{1}$ deve ser escolhido. Vamos provar por contradição e assumir que $\mathcal{X}_{2}$ é o resultado da floresta de caminhos ótimos para $f_{o, \max }^{b k g}$. Da Equação C.2 sabemos que o caminho ótimo para o pixel $u_{2}$ a partir das sementes internas satisfaz $2 \times \omega\left(u_{2}, v_{2}\right)+1>f_{o, \max }^{b k g}\left(\pi_{u_{2}}\right)$ e da hipótese $\omega\left(u_{1}, v_{1}\right)>\omega\left(u_{2}, v_{2}\right)$, podemos concluir que $2 \times \omega\left(u_{1}, v_{1}\right)+1>f_{o, \max }^{b k g}\left(\pi_{u_{2}}\right)$. Mas $\pi_{u_{2}}$ deve passar através de $\mathcal{X}_{1}$ o que implica em uma contradição, pois $f_{o, \max }^{b k g}\left(\pi_{u_{2}}\right) \geq 2 \times \omega\left(u_{1}, v_{1}\right)+1$ pela Equação 6.5.

Similarmente, no segundo caso temos que $\omega\left(u_{1}, v_{1}\right)<\omega\left(u_{2}, v_{2}\right)$ e, de acordo com o Teorema 1 , $\mathcal{X}_{2}$ deve ser escolhido. Assumindo sua negação lógica, obtemos uma contradição como antes por argumentos similares. Assim, o Teorema 1 cumpre-se como queríamos provar.

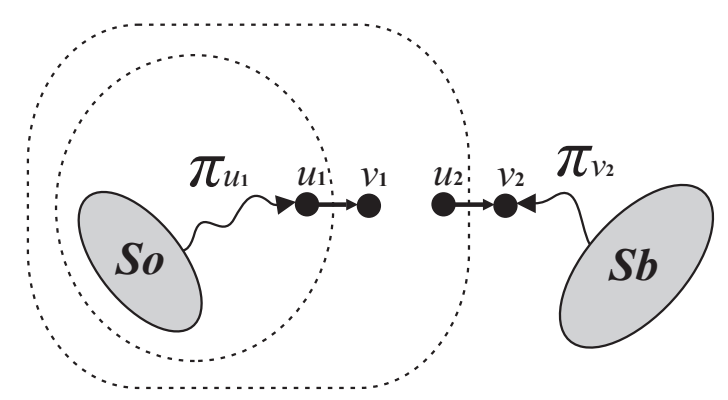

(a)

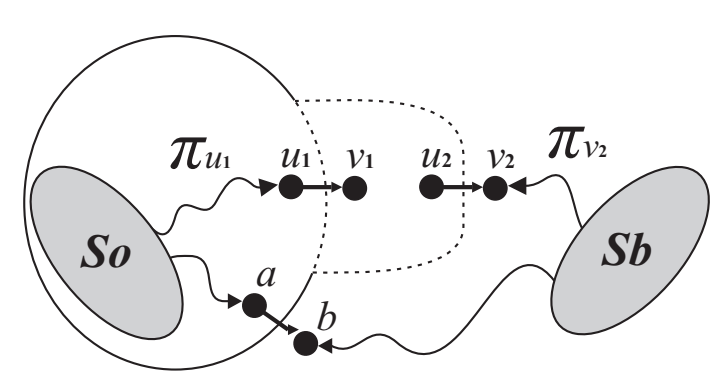

(b)

Figura C.1: São mostrados dois cenários de bordas de corte: (a) Duas bordas candidatas são mostradas com pesos minimos apontando para o exterior, dados por $\omega\left(u_{1}, v_{1}\right)$ e $\omega\left(u_{2}, v_{2}\right)$ respectivamente. (b) Duas possiveis bordas de corte ótimas são mostradas, ambas com valor ótimo na Equação 6.2 dado por $\omega(a, b)$. As linhas pontilhadas representam as partes da borda em questão, $\mathcal{X}_{1}$ e $\mathcal{X}_{2}$, com pesos minimos apontando para o exterior, dados por $\omega\left(u_{1}, v_{1}\right)$ e $\omega\left(u_{2}, v_{2}\right)$ respectivamente.

\section{Teorema 2 (Otimalidade dos cortes internos/externos - caso geral)}

Para dois conjuntos $\mathcal{S}_{o}$ e $\mathcal{S}_{b}$ de sementes dados, qualquer floresta de espalhamento calculada pelo algoritmo da IFT (Algoritmo 1) para a função $f_{o, \max }^{b k g}$ define um corte ótimo que maximiza $E_{o}(\hat{L})$ entre todos os resultados possíveis da segmentação satisfazendo as restrições fortes. Qualquer floresta de espalhamento calculada pelo algoritmo da IFT (Algoritmo 1) para a função $f_{i, \max }^{b k g}$ define um corte ótimo que maximiza $E_{i}(\hat{L})$ entre todos os resultados possíveis da segmentação satisfazendo as restrições fortes. 


\section{Prova:}

Este teorema pode ser verificado, seguindo uma simulação direta do algoritmo da IFT (Algoritmo 1). Nós demonstraremos ele apenas para $f_{o, \max }^{b k g}$, mas a demonstração para $f_{i, \max }^{b k g}$ é essencialmente idêntica.

No início, os pixels sementes, os quais são os mínimos do mapa inicial $V$, são removidos primeiro da fila de prioridade com custo -1 (Equação 6.5), e todos os pixels adjacentes a eles são inseridos na fila. Após isso, nós temos um processo ordenado de crescimento de região das sementes, definindo duas regiões de crescimento

$$
\begin{aligned}
& \mathcal{R}_{o}=\{t \in \mathcal{I} \mid \operatorname{estado}(t)=1 \text { e } L(t)=1\} \text { e } \\
& \mathcal{R}_{b}=\{t \in \mathcal{I} \mid \operatorname{estado}(t)=1 \text { e } L(t)=0\} .
\end{aligned}
$$

A região de crescimento $\mathcal{R}_{o}$ a partir de $\mathcal{S}_{o}$ define uma borda potencial de corte externo, definida por:

$$
\mathcal{X}_{o}=\left\{(a, b) \in \xi \mid a \in \mathcal{R}_{o} \text { e } b \notin \mathcal{R}_{o}\right\} .
$$

Similarmente, nós temos

$$
\mathcal{X}_{b}=\left\{(a, b) \in \xi \mid a \notin \mathcal{R}_{b} \text { e } b \in \mathcal{R}_{b}\right\}
$$

que é uma borda de corte externo para $\mathcal{I} \backslash \mathcal{R}_{b}$.

Inicialmente, temos $\mathcal{R}_{o}=\mathcal{S}_{o}$ e $\mathcal{R}_{b}=\mathcal{S}_{b}$, e os valores do mapa $V$ para os pixels na fila (pixels adjacentes às sementes) serão proporcionais aos pesos das arestas dessas bordas de corte, $\mathcal{X}_{o}$ e $\mathcal{X}_{b}{ }^{1}$.

Na primeira iteração do laço principal (Linha 7), depois de remover todas as sementes, teremos a remoção de um pixel $s$ cujo valor $V(s)$ é mínimo (Linha 8). Se $L(s)=1$ então esse pixel é atribuído a $\mathcal{R}_{o}$ e a aresta $(P(s), s)$ é removida de $\mathcal{X}_{o}$, caso contrário $s$ é atribuído a $\mathcal{R}_{b}$ e $(s, P(s))$ é removido de $\mathcal{X}_{b}$. Note que $V(s)$ é proporcional a $\omega(P(s), s)$ no primeiro caso, e que $V(s)$ é proporcional a $\omega(s, P(s))$ caso contrário. Assim, $s$ é sempre atribuído à região $\left(\mathcal{R}_{o}\right.$ ou $\left.\mathcal{R}_{b}\right)$ com a pior medida de corte externo $\left(\mathcal{X}_{o}\right.$ ou $\left.\mathcal{X}_{b}\right)$, segundo a Equação 6.2.

Vamos supor sem perda de generalidade que $L(s)=1$, de modo que $\mathcal{X}_{o}$ foi o pior corte externo com energia $E^{\prime}=\omega(P(s), s)$. Nesse caso, a remoção de $(P(s), s)$ de $\mathcal{X}_{o}$ é a única operação simples que pode, possivelmente, melhorar esse corte, já que $\omega(P(s), s)=\min _{(p, q) \in \mathcal{X}_{o}} \omega(p, q)$. Como temos uma melhor solução dada por $\mathcal{X}_{b}$, e $\mathcal{X}_{o}$ certamente não é a solução ótima, que maximiza a medida de corte externo (Equação 6.2), podemos executar com segurança essa operação em $\mathcal{X}_{o}^{2}$.

${ }^{1}$ Neste ponto de execução, para todo pixel $t$ na fila, temos que

$$
\begin{aligned}
V(t) & =\min _{(s, t) \in \mathcal{X}_{o}}\{2 \times \omega(s, t)+1\} \quad \text { ou } \\
V(t) & =\min _{(t, s) \in \mathcal{X}_{b}}\{2 \times \omega(t, s)\} .
\end{aligned}
$$

De acordo com a Equação 6.2, as arestas de corte com valores mais baixos definem a energia final. Das fórmulas anteriores, temos que essas arestas com valores mais baixos são consideradas na fila de prioridade. Como o "mais um" serve apenas para propósitos de desambiguação, a fim de dar preferência para caminhos a partir de sementes do fundo, nós essencialmente temos valores $V(t)$ proporcionais às arestas correspondentes do corte.

${ }^{2}$ Como a remoção de $(P(s), s)$ é a única forma de levar $\mathcal{X}_{o}$ a uma melhor solução, não precisamos nos preocupar em voltar atrás dessa decisão. Se ela leva a uma solução ainda pior, não importa, porque temos uma melhor solução 
Depois desse ponto, o processo repete-se nas seguintes iterações, mas pixels $t$ que podem ser alcançados por meio de arestas com pesos mais baixos do que $E^{\prime}$ são também atribuídos a $\mathcal{R}_{o}$, dado que eles levam a cortes de borda ainda piores (isto é, $\omega(P(t), t)<\omega(P(s), s)$ ).

Portanto, como temos um número finito de iterações, e cada iteração garantidamente preserva uma solução futura (não precisamos retroceder de qualquer operação feita), no final, o processo convergirá a um corte que maximiza a Equação 6.2.

de backup armazenada em $\mathcal{X}_{b}$. 


\section{Apêndice D}

\section{Prova de otimalidade do algoritmo GSC-IFT}

A prova da otimalidade do método GSC-IFT em termos da maximização de $E$ (Equação 4.3), entre todos os resultados possíveis da segmentação, satisfazendo as restrições de forma pela convexidade geodésica em estrela (GSC), pode ser verificada seguindo o algoritmo GSC-IFT (Algoritmo 2).

\section{Prova:}

No início, os pixels sementes, os quais são os mínimos do mapa inicial $V$, são removidos primeiro da fila $Q$ de prioridades com custo -1 (Linha 8). De fato, isso deve ser feito, dado que são restrições fortes.

Quando um pixel $s \in \mathcal{S}_{o}$ é removido de $Q$, a subrotina "Conquistar_Caminho" (Algoritmo 3) será chamada. Como $P_{\text {sum }}(s)=n i l$, a subrotina somente definirá estado $(s)=1$, e inserirá todos seus pixels adjacentes na fila $Q$ de prioridade.

Quando um pixel $s \in \mathcal{S}_{b}$ sai da fila $Q$, ele impede a visibilidade de todos seus descendentes em $P_{\text {sum. }}$. Consequentemente, ele e todos seus descendentes tornam-se parte do fundo, e seus arrays de estado são atribuídos para 1 invocando a subrotina "Podar_Árvore" (Algoritmo 4).

Assim, nós temos um processo ordenado de crescimento de região, definindo duas regiões crescentes

$$
\begin{aligned}
& \mathcal{R}_{o}=\{t \in \mathcal{I} \mid \operatorname{estado}(t)=1 \text { e } L(t)=1\}, \quad \text { e } \\
& \mathcal{R}_{b}=\{t \in \mathcal{I} \mid \operatorname{estado}(t)=1 \text { e } L(t)=0\} .
\end{aligned}
$$

A região de crescimento $\mathcal{R}_{o}$ a partir de $\mathcal{S}_{o}$ define uma borda de corte potencial

$$
\mathcal{X}_{o}=\left\{(a, b) \in \xi \mid a \in \mathcal{R}_{o} \text { e } b \notin \mathcal{R}_{o}\right\}
$$

Similarmente, nós temos

$$
\mathcal{X}_{b}=\left\{(a, b) \in \xi \mid a \in \mathcal{R}_{b} \text { e } b \notin \mathcal{R}_{b}\right\}
$$

que é uma borda de corte para $\mathcal{R}_{b}$. 
Os Algoritmos 3 e 4 inserem todos os pixels adjacentes de $\mathcal{R}_{o}$ e $\mathcal{R}_{b}$ na fila $Q$ de prioridade, e os valores do mapa $V$ para esses pixels serão proporcionais aos pesos das arestas nas bordas de corte $\mathcal{X}_{o}$ e $\mathcal{X}_{b}{ }^{1}$.

Na primeira iteração do laço principal (Linha 7), após da remoção de todas as sementes, nós teremos a remoção de um pixel $s$ cujo valor $V(s)$ é mínimo (Linha 8 ). O valor $V(s)$ corresponde à pior aresta $(r, s)$ entre os cortes em $\mathcal{X}_{o}$ e $\mathcal{X}_{b}$, isto é, uma aresta com o menor peso dos cortes

$$
\omega(r, s)=\min _{(t, s) \in \mathcal{X}_{o} \cup \mathcal{X}_{b}}\{\omega(t, s)\}
$$

Assim $s$ é sempre atribuído à região $\left(\mathcal{R}_{o}\right.$ ou $\left.\mathcal{R}_{b}\right)$ com o pior corte $\left(\mathcal{X}_{o}\right.$ ou $\left.\mathcal{X}_{b}\right)$ pela medida $E$ (Equação 4.3).

Suponhamos sem perda de generalidade que $L(s)=1$, tal que $\mathcal{X}_{o}$ é o pior corte com energia $E^{\prime}=\omega(r, s)$. Nesse caso, a remoção de $(r, s)$ a partir de $\mathcal{X}_{o}$ é a única operação simples que pode, possivelmente, melhorar esse corte, dado que $\omega(r, s)=\min _{(t, s) \in \mathcal{X}_{o}} \omega(t, s)$. Em outras palavras, enquanto retivermos $(r, s)$ em $\mathcal{X}_{o}$, sua energia não será maior do que $E^{\prime}=\omega(r, s)$. Como temos uma melhor solução dada por $\mathcal{X}_{b}$, e $\mathcal{X}_{o}$ certamente não é uma solução ótima que maximiza a medida de corte $E$ (Equação 4.3), nós podemos realizar com segurança essa operação em $\mathcal{X}_{o}{ }^{2}$. Mas, temos $L(s)=1$, o que implica que todos os predecessores de $s$ em $P_{\text {sum }}$ devem ser conquistados também como objeto, caso contrário $s$ não será visível a seu centro de estrela mais próximo.

Similarmente, no outro caso nós temos $L(s)=0$, e todos os descendentes de $s$ em $P_{\text {sum }}$ devem também tornar-se parte do fundo $\left(\mathcal{R}_{b}\right)$ devido as restrições de visibilidade.

Essas operações são realizadas por "Conquistar_Caminho" e "Podar_Árvore", respectivamente.

Após esse ponto, o processo repete-se nas seguintes iterações de maneira similar. Portanto, como nós temos um número finito de iterações, e cada iteração garantidamente preserva uma solução futura (não há necessidade de retroceder de qualquer operação feita) no final, esse processo convergirá para um corte que maximiza a Equação 4.3.

\footnotetext{
${ }^{1}$ Para todo pixel $t$ na fila, nós temos

$$
V(t)=\min _{(s, t) \in \mathcal{X}_{o}}\{\omega(s, t)\} \quad \text { ou } \quad V(t)=\min _{(s, t) \in \mathcal{X}_{b}}\{\omega(s, t)\} .
$$
}

De acordo com a Equação 4.3, as arestas de corte com valores mais baixos de $\omega(s, t)$ definem a energia final. Das fórmulas anteriores, nós temos que essas arestas com valores mais baixos são considerados na fila de prioridades.

${ }^{2}$ Como a remoção de $(\mathrm{r}, \mathrm{s})$ é a única forma para levar $\mathcal{X}_{o}$ a uma melhor solução de backup armazenada em $\mathcal{X}_{b}$. 


\section{Apêndice E}

\section{Prova de otimalidade do método GSC-OIFT}

\section{Lema 1}

Para uma dada segmentação $L$, nós temos $\mathcal{C}_{o}(L) \cap \xi_{P_{\text {sum }}}^{o} \neq \emptyset$, se e somente se existe uma violação da restrição de convexidade geodésica em estrela. Nós temos $\mathcal{C}_{i}(L) \cap \xi_{P_{\text {sum }}}^{i} \neq \emptyset$, se e somente se existe uma violação da restrição geodésica em estrela.

\section{Prova:}

Nós demonstraremos isso para $\mathcal{C}_{o}(L) \cap \xi_{P_{\text {sum }}}^{o} \neq \emptyset$. A demonstração para $\mathcal{C}_{i}(L) \cap \xi_{P_{\text {sum }}}^{i} \neq \emptyset$ é essencialmente idêntica. Por definição, uma violação de restrição de convexidade geodésica em estrela para um conjunto de centros $C=\mathcal{S}_{o}$, será dada caso exista um ponto $p \in \mathcal{O}=\{t \mid L(t)=1\}$ que não é visível a $C$ via $\mathcal{O}$ (isto é, existe um pixel $r$ no caminho mais curto ligando $p$ a $C$ em $P_{\text {sum }}$, e $r \notin \mathcal{O})$.

Pelas definições de $\xi_{P_{\text {sum }}}^{o}$ e $\mathcal{C}_{o}(L)$, nós temos $\mathcal{C}_{o}(L) \cap \xi_{P_{\text {sum }}}^{o}=\{(s, t) \in \xi \mid L(s)=1, L(t)=$ 0 e $\left.t=P_{\text {sum }}(s)\right\}$. Para qualquer aresta $(s, t) \in \mathcal{C}_{o} \cap \xi_{P_{\text {sum }}}^{o}$ nós temos $t=P_{\text {sum }}(s)$, o que significa que existe um caminho mais curto $\pi_{s}=\pi_{t} \cdot\langle t, s\rangle$ em $P_{\text {sum }}$ enraizado nas sementes internas $S_{o}$ (isto é, o segmento de reta entre $s$ e $\left.\mathcal{S}_{o}\right)$. Mas $(s, t) \in \mathcal{C}_{o}(L)$ implica que $L(t)=0(t \notin \mathcal{O})$, e por isso $s$ não é visível a $\mathcal{S}_{o}$ por meio de $\pi_{s}=\pi_{t} \cdot\langle t, s\rangle$ em $P_{\text {sum. }}$. Assim, $\mathcal{C}_{o} \cap \xi_{P_{\text {sum }}}^{o} \neq \emptyset$ implica em uma violação da restrição de convexidade geodésica em estrela.

Por outro lado, se nós temos uma violação da restrição de convexidade geodésica em estrela, isso significa que $\exists s \in \mathcal{O}$, ou seja $L(s)=1$, o qual não é visível a $S_{o}$ via o caminho mais curto $\pi_{s}$ em $P_{\text {sum }}$, de modo que existe um pixel $p_{i} \notin \mathcal{O}$ em $\pi_{s}=\left\langle p_{1}, \ldots, p_{i}, \ldots, p_{n}=s\right\rangle, \operatorname{com} P_{\text {sum }}\left(p_{i+1}\right)=p_{i} \mathrm{e}$ $p_{i+1} \in \mathcal{O}$. Por isso, $\left(p_{i+1}, p_{i}\right) \in \mathcal{C}_{o} \cap \xi_{P_{\text {sum }}}^{o}$, o que implica que $\mathcal{C}_{o} \cap \xi_{P_{\text {sum }}}^{o} \neq \emptyset$.

Portanto, nós temos $\mathcal{C}_{o} \cap \xi_{P_{\text {sum }}}^{o} \neq \emptyset$, se e somente se existe uma violação da restrição de convexidade geodésica em estrela. 


\section{Teorema 4 (Otimalidade da borda de corte interno/externo)}

Para um dado grafo ponderado $G=(\mathcal{I}, \xi, \omega)$, considere um grafo ponderado modificado $G^{\prime}=\left(\mathcal{I}, \xi, \omega^{\prime}\right)$, com pesos $\omega^{\prime}(s, t)=-\infty$ para todo $(s, t) \in \xi_{P_{\text {sum }}}^{o}$, e $\omega^{\prime}(s, t)=\omega(s, t)$ caso contrário. Para dois conjuntos $\mathcal{S}_{o}$ e $\mathcal{S}_{b}$ de sementes dados, a segmentação calculada sobre $G^{\prime}$ pelo algoritmo da IFT (Algoritmo 1) para a função $f_{o, \text { max }}^{b k g}$ define um corte ótimo no grafo original $G$, que maximiza $E_{o}(L, G)$ entre todos os resultados possíveis de segmentação satisfazendo as restrições de forma pela convexidade geodésica em estrela, e as restrições de sementes.

Similarmente, a segmentação calculada pelo algoritmo da IFT (Algoritmo 1) para a função $f_{i, \max }^{b k g}$, sobre o grafo modificado $G^{\prime}=\left(\mathcal{I}, \xi, \omega^{\prime}\right)$; com pesos $\omega^{\prime}(s, t)=-\infty$ para todo $(s, t) \in \xi_{P_{\text {sum }}}^{i}$, e $\omega^{\prime}(s, t)=\omega(s, t)$ caso contrário; define um corte ótimo no grafo original $G$, que maximiza $E_{i}(L, G)$ entre todos os resultados possíveis de segmentação satisfazendo as restrições de forma pela convexidade geodésica em estrela, e as restrições de sementes.

\section{Prova:}

Nós provaremos o teorema no caso da função $f_{o \text {,max }}^{b k g}$ O outro caso tem essencialmente uma prova idêntica.

Como nós atribuímos o pior peso possível para todas as arestas $(s, t) \in \xi_{P_{\text {sum }}}^{o}$ em $G^{\prime}$, ou seja $\omega^{\prime}(s, t)=-\infty$, qualquer segmentação $L^{\prime} \operatorname{com} \mathcal{C}_{o}\left(L^{\prime}\right) \cap \xi_{P_{\text {sum }}}^{o} \neq \emptyset$ receberá o pior valor de energia $\left(E_{o}\left(L^{\prime}, G^{\prime}\right)=-\infty\right)^{1}$. Do Teorema 2 , apresentado na Seção 6.2 , nós sabemos que a IFT com $f_{o, \max }^{b k g}$ sobre $G^{\prime}$ maximiza a energia $E_{o}\left(L, G^{\prime}\right)$ no grafo $G^{\prime}$. Consequentemente, isso naturalmente evitará na borda de corte externo qualquer aresta de $\xi_{P_{\text {sum }}}^{o}$. Uma vez que existe sempre uma solução que não viola a restrição GSC (por exemplo, nós poderíamos tomar $\mathcal{O}=\mathcal{S}_{o}$ ), e do Lema 1 , nós temos que a solução calculada não viola a restrição GSC.

Como $\omega(s, t) \geq 0, \forall(s, t) \in \xi$, e do Lema 1 , nós temos que qualquer segmentação candidata $L^{\prime \prime}$ satisfazendo a restrição GSC deve ter $E_{o}\left(L^{\prime \prime}, G^{\prime}\right) \geq 0$. Além disso, como seus pesos em $\mathcal{C}_{o}\left(L^{\prime \prime}\right)$ não foram alterados em $G^{\prime}$, também temos que $E_{o}\left(L^{\prime \prime}, G^{\prime}\right)=E_{o}\left(L^{\prime \prime}, G\right)$. Assim, todos os resultados que satisfazem a restrição GSC foram considerados na otimização, e portanto o Teorema 4 cumpre-se, como queríamos provar.

\footnotetext{
${ }^{1}$ As restrições GSC são incorporadas diretamente no grafo $G^{\prime}$.
} 


\section{Referências Bibliográficas}

Ahuja et al. (1993) R.K. Ahuja, T.L. Magnanti e J.B. Orlin. Network Flows: Theory, Algorithms and Applications. Prentice-Hall. Citado na pág. 22

Allène et al. (2010) C. Allène, J.Y. Audibert, M. Couprie e R. Keriven. Some links between extremum spanning forests, watersheds and min-cuts. Image and Vision Computing, 28(10): 1460-1471. Citado na pág. 2

Audigier e Lotufo (2007) R. Audigier e R.A. Lotufo. Watershed by image foresting transform, tie-zone, and theoretical relationship with other watershed definitions. Em Proceedings of the 8th International Symposium on Mathematical Morphology and its Applications to Signal and Image Processing (ISMM), páginas 277-288, Rio de Janeiro, RJ. MCT/INPE. Citado na pág. 2

Axel et al. (1987) L. Axel, J. Costantini e J. Listerud. Intensity correction in surface-coil MR imaging. American Journal of Roentgenology, 148:418-420. Citado na pág. 5

Bai e Sapiro (2007) X. Bai e G. Sapiro. Distance cut: interactive segmentation and matting of images and videos. Em IEEE International Conference on Image Processing (ICIP), volume 2, páginas II - 249-II - 252, San Antonio, Texas. Citado na pág. 1

Banerjee e Maji (2013) A. Banerjee e P. Maji. Contraharmonic mean based bias field correction in mr images. Em Computer Analysis of Images and Patterns (CAIP), volume 8047, páginas 523-530. Citado na pág. 5

Beucher e Meyer (1993) S. Beucher e F. Meyer. The morphological approach to segmentation: The watershed transformation. Em Mathematical Morphology in Image Processing, chapter 12, páginas 433-481. Marcel Dekker. Citado na pág. 1, 3

Bonilha (2004) L. Bonilha. Dano neuronal em pacientes com epilepsia do lobo temporal medial refratária a tratamento clínico: Estudo quantitativo por ressonância magnética. Tese de Doutorado, Faculty of Medical Sciences, State University of Campinas, Campinas, SP. in Portuguese. Citado na pág. 1

Bonilha et al. (2003) L. Bonilha, E. Kobayashi, G. Castellano, G. Coelho, E. Tinois, F. Cendes e L.M. Li. Texture analysis of hippocampal sclerosis. Epilepsia, 44(12):1546-1550. Citado na pág. 1

Boyes et al. (2008) R.G. Boyes, J.L. Gunter, C. Frost, A.L. Janke, T. Yeatman, D.L.G. Hill, M.A. Bernstein, P.M. Thompson, M.W. Weiner, N. Schuff et al. Intensity non-uniformity correction using N3 on 3-T scanners with multichannel phased array coils. Neuroimage, 39(4):1752-1762. Citado na pág. 5

Boykov e Funka-Lea (2006) Y. Boykov e G. Funka-Lea. Graph cuts and efficient N-D image segmentation. International Journal of Computer Vision, 70(2):109-131. ISSN 0920-5691. doi: http://dx.doi.org/10.1007/s11263-006-7934-5. Citado na pág. 2, 3, 4, 39

Boykov e Jolly (2001) Y.Y. Boykov e M.P. Jolly. Interactive graph cuts for optimal boundary \& region segmentation of objects in N-D images. Em International Conference on Computer Vision (ICCV), volume 1, páginas 105-112. Citado na pág. 2, 3, 4, 5, 7 
Brinkmann et al. (1998) B.H. Brinkmann, A. Manduca e R. A. Robb. Optimized homomorphic unsharp masking for $\mathrm{mr}$ grayscale inhomogeneity correction. Em IEEE Transactions on Medical Imaging, volume 17, páginas 161-171. Citado na pág. 5

Bueno et al. (2001) G. Bueno, O. Musse, F. Heitz e J. P. Armspach. Three-dimensional segmentation of anatomical structures in MR images on large data bases. Magnetic Resonance Imaging, 19:73-88. Citado na pág. 1

Cappabianco et al. (2012) F.A.M. Cappabianco, P.A.V. Miranda, J.S. Ide, C.L. Yasuda e A.X. Falcão. Unraveling the compromise between skull stripping and inhomogeneity correction in $3 \mathrm{~T}$ MR images. Em Conference on Graphics, Patterns and Images (SIBGRAPI 2012), páginas 1-8. Citado na pág. 5,33

Castellano et al. (2004) G. Castellano, L. Bonilha, L.M. Li e F. Cendes. Texture analysis in medical images. Clinical Radiology, in print. Citado na pág. 1

Ciesielski e Udupa (2011) K.C. Ciesielski e J.K. Udupa. Chapter: Region-based segmentation: fuzzy connectedness, graph cut, and other related algorithms in: Biomedical Image Processing. Springer-Verlag. (T.M. Deserno, ed.). Citado na pág. 2, 31

Ciesielski e Udupa (2009) K.C. Ciesielski e J.K. Udupa. Affinity functions: Recognizing essential parameters in fuzzy connectedness based image segmentation. Em Proceedings of SPIE on Medical Imaging: Image Processing, volume 7259. doi: http://dx.doi.org/10.1117/12.811020. Citado na pág. 3

Ciesielski et al. (2007) K.C. Ciesielski, J.K. Udupa, P.K. Saha e Y. Zhuge. Iterative relative fuzzy connectedness for multiple objects with multiple seeds. Computer Vision and Image Understanding, 107(3):160-182. ISSN 1077-3142. Citado na pág. 2, 3, 34, 47

Ciesielski et al. (2011) K.C. Ciesielski, J.K. Udupa, A.X. Falcão e P.A.V. Miranda. Comparison of fuzzy connectedness and graph cut segmentation algorithms. Em Proceedings of SPIE on Medical Imaging: Image Processing, volume 7962, Orlando. doi: 10.1117/12.872522. Citado na pág. 2, 31

Ciesielski et al. (2012a) K.C. Ciesielski, J.K. Udupa, A.X. Falcão e P.A.V. Miranda. A unifying graph-cut image segmentation framework: algorithms it encompasses and equivalences among them. Em Proceedings of SPIE on Medical Imaging, San Diego, California, USA. accepted, to appear. Citado na pág. 2

Ciesielski et al. (2012b) K.C. Ciesielski, J.K. Udupa, A.X. Falcão e P.A.V. Miranda. Fuzzy connectedness image segmentation in graph cut formulation: A linear-time algorithm and a comparative analysis. Journal of Mathematical Imaging and Vision, 44(3):375-398. Citado na pág. 31, 54

Couprie et al. (2010) C. Couprie, L. Grady, L. Najman e H. Talbot. Power watersheds: A unifying graph-based optimization framework. Transactions on Pattern Analysis and Machine Intelligence, 99. ISSN 0162-8828. doi: http://doi.ieeecomputersociety.org/10.1109/TPAMI.2010.200. Citado na pág. $2,3,31,47,54,63$

Cousty et al. (2010) J. Cousty, G. Bertrand, L. Najman e M. Couprie. Watershed cuts: Thinnings, shortest path forests, and topological watersheds. IEEE Transactions on Pattern Analysis and Machine Intelligence, 32:925-939. ISSN 0162-8828. Citado na pág. 2, 3

Dice (1945) L.R. Dice. Measures of the amount of ecologic association between species. Ecology, 26:297-302. Citado na pág. 13

Dijkstra (1959) E.W. Dijkstra. A note on two problems in connexion with graphs. Numerische Mathematik, 1:269-271. Citado na pág. 22 
Falcão (2009) A. X. Falcão. Anotações de aula - mo815: Processamento de imagens usando grafos. Available: http://www.ic.unicamp.br/ afalcao/mo815-grafos/index.html, 2009. Citado na pág. 3

Falcão e Bergo (2004) A.X. Falcão e F.P.G. Bergo. Interactive volume segmentation with differential image foresting transforms. IEEE Transactions on Medical Imaging, 23(9):1100-1108. Citado na pág. 1, 2, 6, 19

Falcão e da Cunha (2001) A.X. Falcão e B.S. da Cunha. Multiscale Shape Representation by Image Foresting Transform. Em Milan Sonka e Kenneth Hanson, editors, Proceedings of SPIE on Medical Imaging, volume 4322, páginas 1091-1100, San Diego, CA. Citado na pág. 19

Falcão et al. (1998) A.X. Falcão, J.K. Udupa, S. Samarasekera, S. Sharma, B.E. Hirsch e R.A. Lotufo. User-steered image segmentation paradigms: Live-wire and live-lane. Graphical Models and Image Processing, 60(4):233-260. Citado na pág. 1, 2, 39

Falcão et al. (2000) A.X. Falcão, J.K. Udupa e F.K. Miyazawa. An ultra-fast user-steered image segmentation paradigm: Live-wire-on-the-fly. IEEE Transactions on Medical Imaging, 19(1):5562. Citado na pág. xvii, 1, 2, 7, 39

Falcão et al. (2001) A.X. Falcão, B.S. da Cunha e R.A. Lotufo. Design of connected operators using the image foresting transform. Em SPIE on Medical Imaging, volume 4322, páginas 468479. Citado na pág. 19

Falcão et al. (2002) A.X. Falcão, L.F. Costa e B.S. da Cunha. Multiscale skeletons by image foresting transform and its applications to neuromorphometry. Pattern Recognition, 35(7):15711582. Citado na pág. 3

Falcão et al. (2004) A.X. Falcão, J. Stolfi e R.A. Lotufo. The image foresting transform: Theory, algorithms, and applications. IEEE Transactions on Pattern Analysis and Machine Intelligence, 26(1):19-29. Citado na pág. 2, 3, 6, 21, 22, 24, 29, 30, 31, 33, 41, 47

Falcão et al. (2008) A.X. Falcão, F.P.G. Bergo, F.O. Favretto, G.C.S. Ruppert, P.A.V. Miranda e F.A.M. Cappabianco. Processamento, Visualização e Análise de Imagens Anatômicas do Cérebro Humano, in: Neurociências e Epilepsia. Ed. Plêiade, São Paulo. ISBN 978-85-7651-080-2. Eds: L.M. Li, P.T. Fernandes, R.J.M. Covolan, F. Cendes. Citado na pág. 66

Gonzalez e Woods (1992) R.C. Gonzalez e R.E. Woods. Digital Image Processing. AddisonWesley, Reading, MA, USA. Citado na pág. 1

Grady (2006) L. Grady. Random walks for image segmentation. IEEE Transactions on Pattern Analysis and Machine Intelligence, 28(11):1768-1783. ISSN 0162-8828. Citado na pág. 2, 7

Gulshan et al. (2010) V. Gulshan, C. Rother, A. Criminisi, A. Blake e A. Zisserman. Geodesic star convexity for interactive image segmentation. Em Proceedings of Computer Vision and Pattern Recognition, páginas 3129-3136. Citado na pág. 7, 15, 17, 36, 49, 50

Hellden (1980) U. Hellden. A test of landsat-2 imagery and digital data for thematic mapping illustrated by an environmental study in northern kenya. Relatório Técnico 47, Lund University, Natural Geography Institute, Sweden. Citado na pág. 13

Herman e Carvalho (2001) G.T. Herman e B.M. Carvalho. Multiseeded segmentation using fuzzy connectedness. IEEE Transactions on Pattern Analysis and Machine Intelligence, 23:460474. Citado na pág. 3

Kass et al. (1987) M. Kass, A. Witkin e D. Terzopoulos. Snakes: Active contour models. International Journal of Computer Vision, 1(4):321-331. Citado na pág. 1, 3 
Labatut e Cherifi (2012) V. Labatut e H. Cherifi. Accuracy measures for the comparison of classifiers. CoRR, abs/1207.3790. Citado na pág. 13

Lézoray e Grady (2012) O. Lézoray e L. Grady. Image Processing and Analysis with Graphs: Theory and Practice. Digital Imaging and Computer Vision. CRC Press / Taylor and Francis. ISBN 978-1-4398-5507-2. Citado na pág. 2

Li et al. (2006) K. Li, X. Wu, D.Z. Chen e M. Sonka. Optimal surface segmentation in volumetric images: A graph-theoretic approach. Transactions Pattern Analysis Machine Intelligence, 28(1): 119-134. Citado na pág. 1

Mansilla e Miranda (2013a) L.A.C. Mansilla e P.A.V. Miranda. Image segmentation by oriented image foresting transform: Handling ties and colored images. Em 18th International Conference on Digital Signal Processing (DSP), páginas 1-6, Santorini, Greece. IEEE. Citado na pág. 7, 29, 46

Mansilla e Miranda (2013b) L.A.C. Mansilla e P.A.V. Miranda. Image segmentation by oriented image foresting transform with geodesic star convexity. Em Computer Analysis of Images and Patterns (CAIP), volume 8047, páginas 572-579, York, UK. Citado na pág. 8

Mansilla et al. (2013a) L.A.C. Mansilla, F.A.M. Cappabianco e P.A.V. Miranda. Image segmentation by image foresting transform with non-smooth connectivity functions. Em XXVI Conference on Graphics, Patterns and Images (SIBGRAPI), páginas 147-154, Arequipa, Perú. IEEE. Citado na pág. 7,29

Mansilla et al. (2013b) L.A.C. Mansilla, M.P. Jackowski e P.A.V. Miranda. Image foresting transform with geodesic star convexity for interactive image segmentation. Em IEEE International Conference on Image Processing (ICIP), páginas 4054-4058, Melbourne, Australia. Citado na pág. 7

Miranda e Falcão (2009) P.A.V. Miranda e A.X. Falcão. Links between image segmentation based on optimum-path forest and minimum cut in graph. Journal of Mathematical Imaging and Vision. ISSN 1573-7683. doi: 10.1007/s10851-009-0159-9. Citado na pág. 2, 7, 22, 24, 31, 32, 39, 49

Miranda e Falcão (2011) P.A.V. Miranda e A.X. Falcão. Elucidating the relations among seeded image segmentation methods and their possible extensions. Em XXIV Conference on Graphics, Patterns and Images (SIBGRAPI), Maceió, AL. Los Alamitos: IEEE Computer Society. Citado na pág. xv, 2

Miranda e Mansilla (2014) P.A.V. Miranda e L.A.C. Mansilla. Oriented image foresting transform segmentation by seed competition. IEEE Transactions on Image Processing, 23(1):389-398. Citado na pág. xviii, $7,29,30,46,47$

Miranda et al. (2008a) P.A.V. Miranda, A.X. Falcão, A. Rocha e F.P.G. Bergo. Object delineation by $\kappa$-connected components. EURASIP Journal on Advances in Signal Processing, páginas 1-14. Citado na pág. 3

Miranda et al. (2008b) P.A.V. Miranda, A.X. Falcão e J.K. Udupa. CLOUDS: A model for synergistic image segmentation. Em Proceedings of the IEEE International Symposium on Biomedical Imaging (ISBI), páginas 209-212, Paris, France. Citado na pág. xv, 6, 66

Miranda et al. (2009) P.A.V. Miranda, A.X. Falcão e J.K. Udupa. Cloud bank: A multiple clouds model and its use in MR brain image segmentation. Em Proceedings of the IEEE International Symposium on Biomedical Imaging (ISBI), páginas 506-509, Boston, MA. Citado na pág. xv, 6, 66

Miranda et al. (2010) P.A.V. Miranda, A.X. Falcão e J.K. Udupa. Synergistic arc-weight estimation for interactive image segmentation using graphs. Computer Vision and Image Understanding, 114(1):85-99. ISSN 1077-3142. Citado na pág. xvii, 3, 4, 5, 36, 38, 47 
Miranda et al. (2011) P.A.V. Miranda, A.X. Falcão e T.V. Spina. The riverbed approach for user-steered image segmentation. Em Proceedings of the International Conference on Image Processing, páginas 3133-3136, Brussels, Belgium. IEEE. doi: 10.1109/ICIP.2011.6116330. Citado na pág. 3, 19

Miranda et al. (2012) P.A.V. Miranda, A.X. Falcão e T.V. Spina. Riverbed: A novel user-steered image segmentation method based on optimum boundary tracking. IEEE Transactions on Image Processing. doi:10.1109/TIP.2012.2188034. Citado na pág. 3, 29

Mortensen e Barrett (1998) E.N. Mortensen e W.A. Barrett. Interactive segmentation with intelligent scissors. Graphical Models and Image Processing, 60:349-384. Citado na pág. 39

Natsume e et al. (2003) J. Natsume e et al. MRI volumetry of the thalamus in temporal, extratemporal, and idiopathic generalized epilepsy. Neurology, 60:1296-1300. Citado na pág. 1

Papa et al. (2009) J.P. Papa, A.X. Falcão e C.T.N. Suzuki. Supervised pattern classification based on optimum-path forest. International Journal of Imaging Systems and Technology, 19(2): 120-131. Citado na pág. 66

Peng et al. (2011) Bo Peng, Lei Zhang, David Zhang e Jian Yang. Image segmentation by iterated region merging with localized graph cuts. Pattern Recognition, 44(10-11):2527-2538. Citado na pág. 2,5

Protiere e Sapiro (2007) A. Protiere e G. Sapiro. Interactive image segmentation via adaptive weighted distances. IEEE Transactions on Image Processing, 16(4):1046-1057. Citado na pág. 1, 3, 4,7

Rauber et al. (2013) P.E. Rauber, A.X. Falcão, T.V. Spina e P.J. Rezende. Interactive segmentation by image foresting transform on superpixel graphs. Em XXVI Conference on Graphics, Patterns and Images (SIBGRAPI), páginas 131-138. IEEE. Citado na pág. 13

Roerdink e Meijster (2000) J.B.T.M. Roerdink e A. Meijster. The watershed transform: Definitions, algorithms and parallelization strategies. Fundamenta Informaticae, 41:187-228. Citado na pág. 2

Rother et al. () C. Rother, V. Kolmogorov, A. Blake e M. Brown. Image and video editing: Grabcut. http://research.microsoft.com/enus/um/cambridge/projects/visionimagevideoediting/segmentation/grabcut.htm. Citado na pág. 48

Rother et al. (2004) C. Rother, V. Kolmogorov e A. Blake. "grabcut": interactive foreground extraction using iterated graph cuts. ACM Transactions on Graphics, 23(3):309-314. ISSN 0730-0301. doi: http://doi.acm.org/10.1145/1015706.1015720. Citado na pág. 3, 5

Saha e Udupa (2001) P.K. Saha e J.K. Udupa. Relative fuzzy connectedness among multiple objects: theory, algorithms, and applications in image segmentation. Computer Vision and Image Understanding, 82(1):42-56. ISSN 1077-3142. Citado na pág. 34, 39

Saha e Udupa (2003) P.K. Saha e J.K. Udupa. Tensor scale-based fuzzy connectedness image segmentation. Em Proceedings of SPIE on Medical Imaging, volume 5032, páginas 1580-1590. Citado na pág. 3

Saha et al. (2000) P.K. Saha, J.K. Udupa e D. Odhner. Scale-based fuzzy connected image segmentation: Theory, algorithms, and validation. Computer Vision and Image Understanding, $77(2): 145-174$. Citado na pág. 3 
Sandock et al. (2000) E.K. Sandock, T.J. O'Brien, C.R. Jack e E.L. So. Significance of cerebellar atrophy in intractable temporal lobe epilepsy: a quantitative MRI study. Epilepsia, 41:1315-1320. Citado na pág. 1

Shi e Malik (2000) J. Shi e J. Malik. Normalized cuts and image segmentation. IEEE Transactions on Pattern Analysis and Machine Intelligence, 22(8):888-905. Citado na pág. 3

Singaraju et al. (2008) D. Singaraju, L. Grady e R. Vidal. Interactive image segmentation via minimization of quadratic energies on directed graphs. Em International Conference on Computer Vision and Pattern Recognition (CVPR), páginas 1-8. Citado na pág. 3

Sinop e Grady (2007) A.K. Sinop e L. Grady. A seeded image segmentation framework unifying graph cuts and random walker which yields a new algorithm. Em 11th International Conference on Computer Vision (ICCV). IEEE Computer Society. ISBN 978-1-4244-1631-8. doi: 10.1109/ ICCV.2007.4408927. Citado na pág. 15

Sled et al. (1998) J.G. Sled, A.P. Zijdenbos e A.C. Evans. A nonparametric method for automatic correction of intensity nonuniformity in mri data. Em IEEE Transactions on Medical Imaging, volume 17 , páginas $87-97$. Citado na pág. 5

Smith (2002) S.M. Smith. Fast robust automated brain extraction. Human Brain Mapping, 17 (3). Citado na pág. xv, 6

Sørensen (1948) T. Sørensen. A Method of Establishing Groups of Equal Amplitude in Plant Sociology Based on Similarity of Species Content and Ist Application to Analyses of the Vegetation on Danish Commons. Det Kongelige Danske Videnskabernes Selskab. Munksgaard. Citado na pág. 13

Spina et al. (2009) T.V. Spina, J.A. Montoya-Zegarra, A.X. Falcão e P.A.V. Miranda. Fast interactive segmentation of natural images using the image foresting transform. Em 16th International Conference on Digital Signal Processing (DSP), páginas 1-8, Santorini, Greece. IEEE. Citado na pág. $3,4,5$

Spina et al. (2012) T.V. Spina, P.A.V. Miranda e A.X. Falcão. Intelligent understanding of user interaction in image segmentation. International Journal of Pattern Recognition and Artificial Intelligence (IJPRAI), 26(2). Citado na pág. 4, 19

Stehman (1997) S.V. Stehman. Selecting and interpreting measures of thematic classification accuracy. Em Remote Sensing of Environment, volume 62, páginas 77-89. Citado na pág. 13

Strand et al. (2013) R. Strand, K.C. Ciesielski, F. Malmberg e P.K. Saha. The minimum barrier distance. Computer Vision and Image Understanding, 117:429-437. Citado na pág. 3

Suetens (2009) P. Suetens. Fundamentals of Medical Imaging. Cambridge University Press. ISBN 0-52-151915-2. Citado na pág. 1

Toennies (2012) K D. Toennies. Guide to Medical Image Analysis: Methods and Algorithms. Springer-Verlag. ISBN 978-1-4471-2750-5. Citado na pág. 1

Torres et al. (2004) R.S. Torres, A.X. Falcão e L.F. Costa. A graph-based approach for multiscale shape analysis. Pattern Recognition, 37(6):1163-1174. Citado na pág. 3

Udupa et al. (2002) J.K. Udupa, P.K. Saha e R.A. Lotufo. Relative fuzzy connectedness and object definition: Theory, algorithms, and applications in image segmentation. IEEE Transactions on Pattern Analysis and Machine Intelligence, 24:1485-1500. Citado na pág. 2

van Rijsbergen (1979) C.J. van Rijsbergen. Information retrieval. Wiley Inter-science, London, second ed. Citado na pág. 13 
Vincent e Soille (1991) L. Vincent e P. Soille. Watersheds in digital spaces: An efficient algorithm based on immersion simulations. IEEE Transactions on Pattern Analysis and Machine Intelligence, 13(6). Citado na pág. 2

Wang e Siskind (2001) S. Wang e J.M. Siskind. Image segmentation with minimum mean cut. Em IEEE International Conference on Computer Vision (ICCV), volume 1, páginas 517-524. Citado na pág. 3

W.Yang et al. (2010) W.Yang, J.Cai, J. Zheng e J. Luo. User-friendly interactive image segmentation through unified combinatorial user inputs. IEEE Transactions on Image Processing, 19 (9):2470-2479. Citado na pág. 2 


\section{Îndice Remissivo}

acurácia

coeficiente de Dice, 13

definição, 13

caminho

ótimo, 21

ótimo completo, 22

definição, 11

convexidade geodésica em estrela

convexidade em estrela, 49

segmentação, 51

algoritmo, 52

experimentos, 54

corte em grafo

definição, 12

energia, 31

energia externa, 39, 59

energia interna, 39, 59

função de conexidade

definição, 20

não suave, 29

classificação, 29

exemplo, 30

inomogeneidade, 36

segmentação, 33, 41, 45, 59

suave, 21

grafo

árvore, 12

acíclico, 11

caminho, 11

conexo, 12

corte, 12

de imagem, 12

definição, 10

direcionado, 11

floresta, 12

ponderado, 11

relação de adjacência, 11

subgrafo, 11

GSC-IFT, veja convexidade geodésica em estrela
GSC-OIFT, veja transformada imagem-floresta orientada com convexidade geodésica em estrela

IFT, veja transformada imagem-floresta

IFT-SC, veja transformada imagem-floresta por competição de sementes

imagem digital

amostragem, 9

definição, 9

multibanda, 10

multidimensional, 10

quantização, 10

rotulada, 10

tons de cinza, 9

marcadores

por um usuário robô, 17

por erosão, 15

objeto, 13

OIFT, veja transformada imagem-floresta orientada

transformada imagem-floresta

caminho ótimo, 21

caminho ótimo completo, 22

competição de sementes, 22

algoritmo, 22

desempate, 24

empate, 24

exemplo, 23

definição, 19

função de conexidade, 20

não suave, 33

suave, 21

polaridade de borda, 39

polaridade de borda com restrições de forma, 59

restrições de forma, 51

transformada imagem-floresta orientada

convexidade geodésica em estrela

experimentos, 62

segmentação, 59 
definição, 39

segmentação, 41, 45

experimentos, 42,46 